Algoritmos evolutivos para modelos de mistura de gaussianas em problemas com e sem restrições 

SERVIÇO DE PÓS-GRADUAÇÃO DO ICMC-USP

Data de Depósito:

Assinatura:

\title{
Algoritmos evolutivos para modelos de mistura de gaussianas em problemas com e sem restrições ${ }^{1}$
}

\author{
Thiago Ferreira Covões
}

Orientador: Prof. Dr. Eduardo Raul Hruschka

Tese apresentada ao Instituto de Ciências Matemáticas e de Computação - ICMC-USP, como parte dos requisitos para obtenção do título de Doutor em Ciências - Ciências de Computação e Matemática Computacional. VERSÃO REVISADA

USP - São Carlos

Fevereiro de 2015

\footnotetext{
${ }^{1}$ Trabalho realizado com suporte financeiro da FAPESP, Processo no 2009/17795-0
} 
Ficha catalográfica elaborada pela Biblioteca Prof. Achille Bassi e Seção Técnica de Informática, ICMC/USP, com os dados fornecidos pelo(a) autor(a)

Covões, Thiago Ferreira
Algoritmos evolutivos para modelos de mistura de
gaussianas em problemas com e sem restrições /
Thiago Ferreira Covões; orientador Eduardo Raul
Hruschka. -- São Carlos, 2015.
138 p.
Tese (Doutorado - Programa de Pós-Graduação em
Ciências de Computação e Matemática Computacional) --
Instituto de Ciências Matemáticas e de Computação,
Universidade de São Paulo, 2015.
1. Agrupamento de dados semissupervisionado. 2.
Algoritmos evolutivos. 3. Aprendizado de máquina.
I. Hruschka, Eduardo Raul, orient. II. Título.





\section{Agradecimentos}

Sou muito grato ao meu orientador Professor Eduardo Raul Hruschka, pessoa por quem tenho enorme admiração, que foi além de um excepcional orientador, um amigo com quem pude contar durante os longos anos da pós-graduação. Em especial, gostaria de agradecer também a sua família, Luciana e Catharina, por todo o carinho e paciência que tiveram comigo durante o período do meu doutorado-sanduíche. Tenho uma dívida eterna com vocês.

Agradeço ao Professor Joydeep Ghosh, meu supervisor durante o doutorado sanduíche que realizei na Universidade do Texas em Austin, por ter me aceitado em seu grupo e pelos ensinamentos durante o desenvolvimento do trabalho.

À Debora, minha namorada, por ter sido paciente e compreensiva ao longo destes anos e por estar sempre ao meu lado, tanto nos momentos bons quanto nos ruins.

À minha família, meus pais José e Rosana, por todo o apoio durante todos os meus anos de vida e por me proverem condições para eu ter conseguido chegar até aqui.

Gostaria também de agradecer aos amigos que fiz no ICMC, em especial a André Rossi, Bruno Feres, Bruno Nogueira, Danilo Horta, Jonathan Andrade, Lucas Vendramin, Luís Paulo, Luiz Coletta, Marcelo Oliveira, Márcio Basgalupp, Merley Conrado, Murilo Naldi, Pablo Andretta, Paulo Henrique, Renato Ramos, Ricardo Cerri, Ricardo Rios, Roberto Sadao, Rodrigo Barros, Rodrigo Calvo, Rosane Maffei, Tiago Silva e Victor Laguna, pelos momentos de discussão sobre assuntos relacionados a este trabalho, pelos momentos de companheirismo e confraternização e por estarem sempre dispostos a ajudar.

A todos os funcionários do ICMC da USP, pela competência e dedicação.

À FAPESP pelo apoio financeiro para a realização deste trabalho (Processo 2009/17795-0).

Ao CNPq pelo apoio financeiro para a realização do doutorado sanduíche na Universidade do Texas (Bolsa SWE - Processo 200372/2011-4). 



\section{Resumo}

Nesta tese, são estudados algoritmos para agrupamento de dados, com particular ênfase em Agrupamento de Dados com Restrições, no qual, além dos objetos a serem agrupados, são fornecidos pelo usuário algumas informações sobre o agrupamento desejado. Como fundamentação para o agrupamento, são considerados os modelos de mistura finitos, em especial, com componentes gaussianos, usualmente chamados de modelos de mistura de gaussianas. Dentre os principais problemas que os algoritmos desenvolvidos nesta tese de doutorado buscam tratar destacam-se: (i) estimar parâmetros de modelo de mistura de gaussianas; (ii) como incorporar, de forma eficiente, restrições no processo de aprendizado de forma que tanto os dados quanto as restrições possam ser adicionadas de forma online; (iii) estimar, via restrições derivadas de conceitos pré-determinados sobre os objetos (usualmente chamados de classes), o número de grupos destes conceitos. Como ferramenta para auxiliar no desenvolvimento de soluções para tais problemas, foram utilizados algoritmos evolutivos que operam com mais de uma solução simultaneamente, além de utilizarem informações de soluções anteriores para guiar o processo de busca. Especificamente, foi desenvolvido um algoritmo evolutivo baseado na divisão e união de componentes para a estimação dos parâmetros de um modelo de mistura de gaussianas. Este algoritmo foi comparado com o algoritmo do mesmo gênero considerado estado-da-arte na literatura, apresentando resultados competitivos e necessitando de menos parâmetros e um menor custo computacional. Nesta tese, foram desenvolvidos dois algoritmos que incorporam as restrições no processo de agrupamento de forma online. Ambos os algoritmos são baseados em algoritmos bem-conhecidos na literatura e apresentaram, em comparações empíricas, resultados melhores que seus antecessores. Finalmente, foram propostos dois algoritmos para se estimar o número de grupos por classe. Ambos os algoritmos foram comparados com algoritmos reconhecidos na literatura de agrupamento de dados com restrições, e apresentaram resultados competitivos ou melhores que estes. A estimação bem sucedida do número de grupos por classe pode auxiliar em diversas tarefas de mineração de dados, desde a sumarização dos dados até a decomposição de problemas de classificação em sub-problemas potencialmente mais simples. 



\section{Abstract}

In the last decade, researchers have been giving considerable attention to the field of Constrained Clustering. Algorithms in this field assume that along with the objects to be clustered, the user also provides some constraints about which kind of clustering (s)he prefers. In this thesis, two scenarios are studied: clustering with and without constraints. The developments are based on finite mixture models, namely, models with Gaussian components, which are usually called Gaussian Mixture Models (GMMs). In this context the main problems addressed are: (i) parameter estimation of GMMs; (ii) efficiently integrating constraints in the learning process allowing both constraints and the data to be added in the modeling in an online fashion; (iii) estimating, by using constraints derived from pre-determined concepts (usually named classes), the number of clusters per concept. Evolutionary algorithms were adopted to develop solutions for such problems. These algorithms analyze more than one solution simultaneously and use information provided by previous solutions to guide the search process. Specifically, an evolutionary algorithm based on procedures that perform splitting and merging of components to estimate the parameters of a GMM was developed. This algorithm was compared to an algorithm considered as the state-of-the-art in the literature, obtaining competitive results while requiring less parameters and being more computationally efficient. Besides the aforementioned contributions, two algorithms for online constrained clustering were developed. Both algorithms are based on well known algorithms from the literature and get better results than their predecessors. Finally, two algorithms to estimate the number of clusters per class were also developed. Both algorithms were compared to well established algorithms from the literature of constrained clustering, and obtained equal or better results than the ones obtained by the contenders. The successful estimation of the number of clusters per class is helpful to a variety of data mining tasks, such as data summarization and problem decomposition of challenging classification problems. 



\section{Conteúdo}

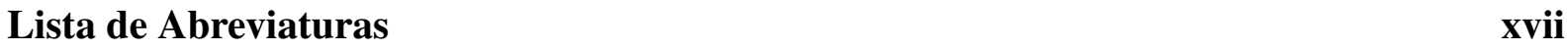

Lista de Figuras $\quad$ xix

Lista de Tabelas $\quad$ xxi

Lista de Algoritmos $\quad$ xxiii

$\begin{array}{ll}\text { Lista de Símbolos } & \text { Xxv }\end{array}$

1 Introdução 1

1.1 Aprendizado Supervisionado . . . . . . . . . . . . . . . . 2

1.2 Aprendizado Não Supervisionado . . . . . . . . . . . . . . . . 3

1.3 Aprendizado Semissupervisionado . . . . . . . . . . . . . 5

1.4 Motivações e Hipóteses . . . . . . . . . . . . . . . . . . . 6

1.5 Organização da Tese . . . . . . . . . . . . . . . . . . . 8

2 Modelos de Mistura de Gaussianas $\quad 11$

2.1 Considerações Iniciais $\ldots \ldots \ldots$. . . . . . . . . . . . . . . . 11

2.2 Estimação de Parâmetros . . . . . . . . . . . . . . . . . . . . . . . . 12

2.3 Procedimentos para União e Divisão de Componentes em Gaussian Mixture Models . . . . . . . . . . . . . . . . . . . 15

2.3.1 União de Componentes . . . . . . . . . . . . . . . . . . . . 15

2.3.2 Divisão de Componentes . . . . . . . . . . . . . . . . . . 17

2.4 Algoritmos Evolutivos _. . . . . . . . . . . . . . . . . . . . . 19

2.4.1 Algoritmos Evolutivos Baseados em $K$-means . . . . . . . . . . . . . . 22

2.4.2 Algoritmos Evolutivos Baseados em Expectation Maximization para Gaussian Mixture Models . . . . . . . . . . . . . . . . . . . . 26

2.5 Considerações Finais . . . . . . . . . . . . . . . . . . . . 31 


\section{Algoritmos Evolutivos para Modelos de Mistura de Gaussianas Não Supervisiona-}

dos $\quad 35$

3.1 Considerações Iniciais $\ldots \ldots . \ldots$. . . . . . . . . . . . . . . 35

3.2 Evolutionary Split \& Merge Algorithm for Expectation Maximization . . . . . . 36

3.2 .1 Inicialização . . . . . . . . . . . . . . . . . . . . . 37

3.2.2 Refinamento . . . . . . . . . . . . . . . 37

3.2.3 Seleção de Indivíduos para Mutação e Sobrevivência . . . . . . . . . 38

3.2 .4 Operadores de Mutação . . . . . . . . . . . . . . . . . . 38

3.2 .5 Resumo de Variantes . . . . . . . . . . . . . . . . . . . . . . . 47

3.3 Notas sobre Complexidade Computacional . . . . . . . . . . . . . . . . . . . 47

3.4 Avaliação Empírica . . . . . . . . . . . . . . . . . . . . . . . . . . . . . . 48

3.4.1 Comparação entre Variantes do ESM-EM . . . . . . . . . . . . . . . . 50

3.4.2 Comparação entre ESM-EM, GA-EM e OMR-EM . . . . . . . . . . . 54

3.5 Considerações Finais . . . . . . . . . . . . . . . . . . . . . 57

4 Agrupamento de Dados com Restrições 59

4.1 Considerações Iniciais ． . . . . . . . . . . . . . . . . . . . . . . . . . . . 59

4.2 Restrições Must-Link e Cannot-Link e suas Propriedades ～. . . . . . . . . . . 60

4.3 Algoritmos que Utilizam Restrições Must-Link e Cannot-Link . . . . . . . . 63

4.3.1 Algoritmos Baseados no K-means . . . . . . . . . . . . . . . . . 64

4.3.2 Algoritmos Baseados no Expectation Maximization para Gaussian Mixture Models . . . . . . . . . . . . . . . . . . . . . . . 73

4.4 Considerações Finais . . . . . . . . . . . . . . . . . . . . 75

5 Aprendizado Competitivo para Agrupamento de Dados com Restrições 77

5.1 Considerações Inciais . . . . . . . . . . . . . . . . . . . . . 77

5.2 Aprendizado Competitivo . . . . . . . . . . . . . . . . 78

5.3 Online Linear Constrained Vector Quantization Error . . . . . . . . . . . . . . 79

5.4 Constrained Rival Penalized Competitive Learning . . . . . . . . . . . . . . . 79

5.5 Avaliação Empírica . . . . . . . . . . . . . . . . . . . . . . . 81

5.6 Considerações Finais $\ldots \ldots \ldots$. . . . . . . . . . . . . . . . 86

6 Modelando Múltiplos Grupos por Classe $\quad 87$

6.1 Considerações Iniciais _ . . . . . . . . . . . . . . . . . . . . . 87

6.2 Descrição do Algoritmo . . . . . . . . . . . . . . . . . . . . . . . . . . . 89

6.3 Análise Empírica . . . . . . . . . . . . . . . . . . . . . . . . . . . . . 92

6.3.1 Comparando as Partições dos Dados . . . . . . . . . . . . . . 93

6.3.2 Avaliando o Erro de Classificação . . . . . . . . . . . . . . . . . . 93

6.4 Considerações Finais . . . . . . . . . . . . . . . . . . . . . . . . . . . . . . . 94 
7 Algoritmo Evolutivo para Modelos de Mistura de Gaussianas que Utiliza Restri$\begin{array}{ll}\text { ções } & 97\end{array}$

7.1 Considerações Inciais . . . . . . . . . . . . . . . . . . . . . . . 97

7.2 Feasible-Infeasible Two-Population . . . . . . . . . . . . . 98

7.3 Feasible-Infeasible Evolutionary Create \& Eliminate Algorithm for Expectation Maximization ......................... 99

7.3 .1 Inicialização . . . . . . . . . . . . . . . . . . . . . 99

7.3.2 Função Objetivo . . . . . . . . . . . . . . . . . . . . . 101

7.3.3 Operadores de Mutação . . . . . . . . . . . . . . . . . . . . . 101

7.3.4 Seleção de Sobreviventes . . . . . . . . . . . . . . . . . . . . . 103

7.3.5 Cuidados Adicionais . . . . . . . . . . . . . . . . 103

7.4 Exemplo Pedagógico . . . . . . . . . . . . . . . . . . . . 103

7.5 Análise Empírica . . . . . . . . . . . . . . . . . . . . . . . 108

7.5.1 Comparando as Partições dos Dados . . . . . . . . . . . . . . . . 109

7.5.2 Avaliando o Erro de Classificação . . . . . . . . . . . . . . . . . . . . 110

7.6 Considerações Finais . . . . . . . . . . . . . . . . . . . . . 112

8 Conclusões e Trabalhos futuros $\quad 115$

8.1 Trabalhos Futuros . . . . . . . . . . . . . . . . . . . . . 117

8.1.1 Implementações Paralelas e Distribuídas . . . . . . . . . . . . . . . . 117

8.1.2 Extensão de Análises Empíricas . . . . . . . . . . . . . . . . . . 118

8.1.3 Melhorias nos Algoritmos e Análises de Aprendizado Competitivo . . . 118

$\begin{array}{ll}\text { Referências Bibliográficas } & 121\end{array}$ 



\section{Lista de Abreviaturas}

ADR............ Agrupamento de Dados com Restrições

AE $\ldots \ldots \ldots \ldots \ldots$ Algoritmo Evolutivo

ARI .............. Adjusted Rand Index

BIC ............ Bayesian Information Criterion

CL .............. Cannot-Link

Cop-HGA ........ Constrained Hybrid Genetic Algorithm

C-RPCL.......... Constrained Rival Penalized Competitive Learning

CVQE ............ Constrained Vector Quantization Error

EM............ Expectation Maximization

ECE-EM .......... Evolutionary Create \& Eliminate Algorithm for Expectation Maximization

ESM-EM ......... Evolutionary Split \& Merge Algorithm for Expectation Maximization

F-EAC .......... Fast Evolutionary Algorithm for Clustering

FI2POP........... Feasible-Infeasible Two-Population

FIECE-EM ........ Feasible-Infeasible Evolutionary Create \& Eliminate Algorithm for Expectation Maximization

GA-EM........... Genetic-based Expectation Maximization

GAKREM ......... Genetic Algorithm K-means Logarithmic Regression Expectation Maximization 
GMM

Gaussian Mixture Model

HLL Held-out Log-Likelihood

KDD Knowledge Discovery in Databases

LCVQE Linear Constrained Vector Quantization Error

LKD Local Kullback Divergence

MCCK Multiple Clusters per Class K-means

MCP Multiple Components Parameterized

MD Mineração de Dados

MG Merge Groups

MDL Minimum Description Length

ML Must-Link

MLE Maximum Likelihood Estimate

MPCK-Means Metric Pairwise Constrained K-Means

NMI . Normalized Mutual Information

O-LCVQE Online Linear Constrained Vector Quantization Error

OMR-EM Ordered Multiple Runs of Expectation Maximization

PLL Partial Log-Likelihood

RPCL Rival Penalized Competitive Learning

SS Silhueta Simplificada

SVD Singular Value Decomposition

SVDS Singular Value Decomposition Splitting

SM . Split and Merge

VS. Variance based Splitting 


\section{Lista de Figuras}

3.1 Exemplo da aplicação do operador Merge Groups. . . . . . . . . . . . . . . . . 44

3.2 Exemplo da aplicação do operadores de divisão. . . . . . . . . . . . . . . . . 46

3.3 Gráfico de dispersão de bases de dados utilizadas nos experimentos. . . . . . . . 49

3.4 Exemplo dos critérios de separação para cada par de componentes gaussianas de um Gaussian Mixture Model (GMM). . . . . . . . . . . . . . . . . . . . 50

3.5 Diferenças médias de Held-out Log-Likelihood (HLL) obtidas pelas diferentes variantes do ESM-EM e a variante Singular Value Decomposition (SVD) nas bases de dados $c$-separation com $K=4$ (quanto menor o valor melhor a variante). Resultados para as bases de dados $\operatorname{com} c=1$ foram omitidos devido ao fato de que nenhuma diferença foi observada. . . . . . . . . . . . . . . . . 52

3.6 Diferenças médias de HLL obtidas pelas diferentes variantes do ESM-EM e a variante SVD nas bases de dados $\omega$-separation com $K=4$ (quanto menor o valor melhor a variante).

3.7 Diferenças médias de HLL obtidas pelas diferentes variantes do ESM-EM e a variante SVD nas bases de dados $c$-separation com $K=12$ (quanto menor o valor melhor a variante).

3.8 Diferenças médias de HLL obtidas pelas diferentes variantes do ESM-EM e a variante SVD nas bases de dados $\omega$-separation com $K=12$ (quanto menor o valor melhor a variante)

3.9 Boxplot dos valores de MDL (divididos pelo número de objetos) obtidos por cada algoritmo. O eixo $x$ indica o número de repetições $\left(n_{p}\right)$ realizadas pelo Ordered Multiple Runs of Expectation Maximization (OMR-EM). . . . . . . . 57

4.1 Exemplo de distinção entre grupos e classes. . . . . . . . . . . . . . . . . . . 62

4.2 Base de dados Iris. . . . . . . . . . . . . . . . . . . . . . . . 64

5.1 Resultados detalhados na base de dados Pendigits. . . . . . . . . . . . . . . 85

6.1 Exemplo do efeito outlier Klein et al. (2002). . . . . . . . . . . . . . . . 88 
6.2 Valores médios de Adjusted Rand Index (ARI) obtidos para cada quantidade de objetos rotulados por classe. . . . . . . . . . . . . . . . 94

$7.1 \quad$ Base de dados sintética. . . . . . . . . . . . . . . . . . . . . 104

7.2 Indivíduo infeasible da $1^{\text {a }}$ geração. . . . . . . . . . . . . . . . . . . . 105

7.3 Indivíduo infeasible que origina o primeiro indivíduo feasible. Objetos que estão violando restrições são indicados pelas setas. . . . . . . . . . . . . 105

7.4 Indivíduo feasible gerado após a criação de novos grupos. . . . . . . . . . . . 106

7.5 Indivíduo feasible após remoção de grupos vazios. . . . . . . . . . . . . . 106

7.6 Indivíduo feasible com grupos a serem removidos indicados. . . . . . . . . . . 107

7.7 Indivíduo infeasible gerado a partir de indivíduo feasible . . . . . . . . . . . . 107

7.8 Modelo final obtido pelo Feasible-Infeasible Evolutionary Create \& Eliminate Algorithm for Expectation Maximization (FIECE-EM) após convergência. . . . 108

7.9 Valores médios de ARI obtidos para cada quantidade de objetos rotulados por classe. . . . . . . . . . . . . . . . . . . . . 111

8.1 Fluxo do desenvolvimento desta tese. . . . . . . . . . . . . . . 115 


\section{Lista de Tabelas}

1.1 Base de dados representada no formato atributo-valor. . . . . . . . . . . . . . . 2

3.1 Sumário das variantes desenvolvidas . . . . . . . . . . . . . . . . 47

3.2 Sumário das bases de dados. Número correto de grupos $(K)$ para a base de dados Pendigits não é conhecido. . . . . . . . . . . . . . . . . . . 50

3.3 Valores de critérios de separação $c$ e $\omega$ para os dados gerados. . . . . . . . . . 51

3.4 Ranking médio de cada variante considerando o valor de adequabilidade (MDL), número de iterações do EM (EMS), diferença absoluta entre número de grupos predito real $(\approx \mathrm{K})$. A última coluna apresenta a média dos rankings de cada algoritmo. . . . . . . . . . . . . . . . . 51

3.5 Ranking médio obtido por cada algoritmo - considerando o valor de Minimum Description Length (MDL) obtido pelo melhor modelo para cada valor de $n_{p} . \quad$. 56

5.1 Sumário das bases de dados utilizadas. . . . . . . . . . . . . . . . . 83

5.2 Diferenças entre valores de Normalized Mutual Information (NMI) obtidos pelo algoritmo Constrained Rival Penalized Competitive Learning (C-RPCL) e o Linear Constrained Vector Quantization Error (LCVQE) (quanto maior melhor) — \#OR significa número de objetos rotulados usados para gerar as restrições. .

5.3 Diferenças entre valores de NMI obtidos pelo algoritmo Online Linear Constrained Vector Quantization Error (O-LCVQE) e o LCVQE (quanto maior melhor) — \#OR significa número de objetos rotulados usados para gerar as restrições. 83

5.4 Médias e desvios-padrão dos valores de NMI para diferentes quantidades de objetos rotulados (\#OR) - Pendigits.

5.5 Diferenças entre o número de restrições violadas pelos algoritmos online e o LCVQE (quanto menor melhor) . . . . . . . . . . . . . . . . . . . . 84

5.6 Resultados obtidos na base de dados do KDDCUP'99 de Detecção de intrusão em redes (desvios padrão em parênteses) . . . . . . . . . . . . . . . . . . . . 86

6.1 Bases de dados usadas nos experimentos. . . . . . . . . . . . . . . . . . . 92 
6.2 Número médio de grupos por classe estimados pelo Multiple Clusters per Class $K$-means (MCCK) (considerando todas as quantidades de objetos rotulados). . .

6.3 Taxas de erro médias (\%) para diferentes quantidades de objetos rotulados por classe $(\# \mathrm{OR}) . \ldots \ldots$. . . . . . . . . . . . . . . . . . . . . . . 95

7.1 Bases de dados usadas nos experimentos. . . . . . . . . . . . . . . . 109

7.2 Número médio de grupos por classe estimados pelo FIECE-EM e Multiple Components Parameterized (MCP) (considerando todas as quantidades de objetos rotulados) . . . . . . . . . . . . . . . . . . . 110

7.3 Taxas de erro médias (\%) para diferentes quantidades de objetos rotulados por classe (\# OR) . . . . . . . . . . . . . . . . . . . . . . . . . 112

7.4 Tabela de vitórias/empates/derrotas entre os algoritmos para diferentes quantidades de objetos rotulados por classe (\# OR) considerando as taxas de erro médias. . . . . . . . . . . . . . . . . . . . . . 112 


\section{Lista de Algoritmos}

$1 \quad$ Ordered Multiple Runs of Expectation Maximization (OMR-EM). . . . . . . . . . 14

2 Operador de mutação MO1. . . . . . . . . . . . . . . . . . . . . . . 24

3 Operador de mutação MO2. . . . . . . . . . . . . . . . . . 25

4 Fast Evolutionary Algorithm for Clustering. . . . . . . . . . . . . . . . . 26

5 Algoritmo Genetic-based Expectation Maximization. . . . . . . . . . . . . . . . 30

6 Genetic Algorithm K-means Logarithmic Regression Expectation Maximization. 32

7 Evolutionary Split \& Merge Algorithm for Expectation Maximization (ESM-EM). 37

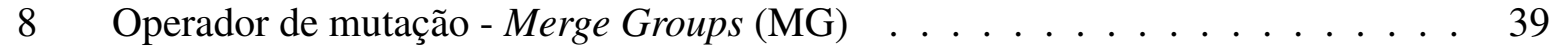

9 Operador de mutação - Singular Value Decomposition Splitting (SVDS) . . . . . 40

10 Operador de mutação - Variance based Splitting (VS) . . . . . . . . . . . . . . . 41

11 Algoritmo K-means. . . . . . . . . . . . . . . . . . . 65

12 Operador de mutação One-step Constrained K-means. . . . . . . . . . . . . . . 72

13 Online Linear Constrained Vector Quantization Error (O-LCVQE). . . . . . . . 80

14 Constrained Rival Penalized Competitive Learning (C-RPCL). . . . . . . . . . . 82

15 Algoritmo MCCK .......................... 91

16 Feasible-Infeasible Evolutionary Create \& Eliminate Algorithm for Expectation Maximization (FIECE-EM). . . . . . . . . . . . . . . . . . 100

17 Operador de mutação que elimina componentes. . . . . . . . . . . . . . . . 102

18 Operador de mutação que cria componentes. . . . . . . . . . . . . . . . . . 102 



\title{
Lista de Símbolos
} \\ Conjunto de dados \\ $\mathbf{x}_{i}$ \\ $\mathbf{A}^{T}$ \\ pertencem \\ $\|\mathbf{a}-\mathbf{b}\|_{\mathbf{A}} \quad$ Distância Euclidiana entre $\mathbf{a}$ e $\mathbf{b}$ parametrizada por $\mathbf{A}$
}


$\mu_{i}$

$\Sigma_{i}$

$\pi_{i}$

$\gamma\left(z_{j i}\right)$

$\Psi$

$\mathcal{P}$

$P$

$\mathcal{P}_{i}^{(g)}$

$K_{\max }$
Média da $i$-ésima distribuição gaussiana

Matriz de covariância da $i$-ésima distribuição gaussiana

Coeficiente de mistura da $i$-ésima distribuição gaussiana

Responsabilidade da $i$-ésima distribuição gaussiana em explicar $\mathbf{x}_{j}$

Conjunto de parâmetros do Gaussian Mixture Model

População de indivíduos de um Algoritmo Evolutivo

Tamanho da população

$i$-ésimo indivíduo da população da $g$-ésima geração

Valor máximo de número de grupos permitido 


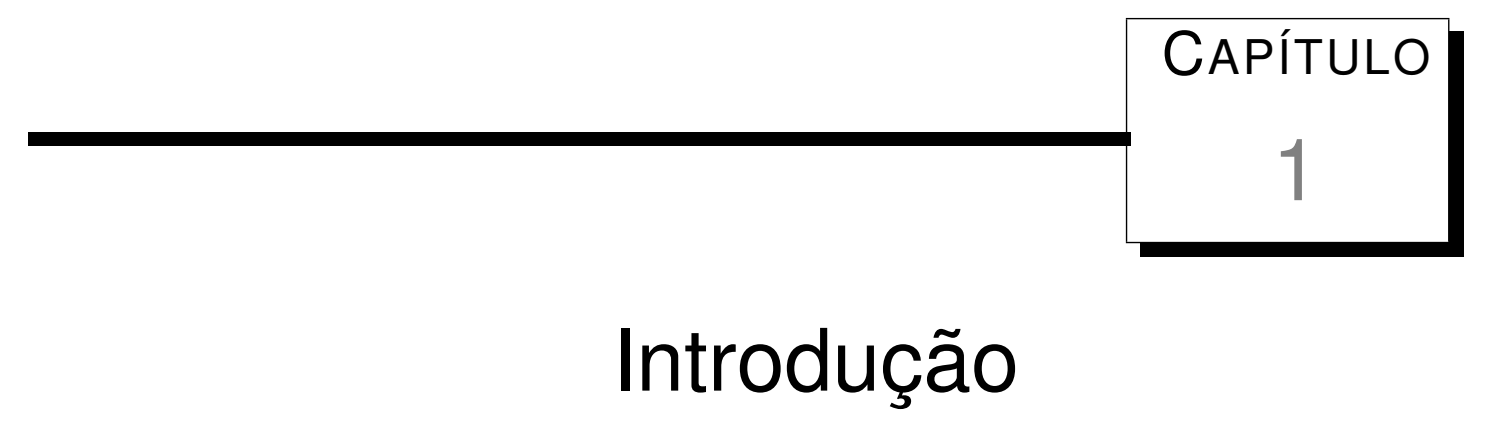

Com a evolução da tecnologia nas últimas décadas, a velocidade de processamento e a capacidade de armazenamento de dispositivos computacionais aumentaram de maneira significativa. Por conta disso, dados em quantidades abundantes têm sido gerados, coletados e processados. Como exemplos notáveis, pode-se mencionar a informatização de transações comerciais, o armazenamento de imagens recuperadas de satélite e a popularização da World Wide Web. Tal abundância de dados torna inviável a utilização de métodos tradicionais de análises de dados (Fayyad et al., 1996b; Chen e Zhang, 2014). Surgiu, então, uma necessidade de técnicas que possam, de forma eficaz e automática, analisar grandes conjuntos de dados, com o objetivo de encontrar conhecimento.

O processo pelo qual se busca extrair conhecimento de um conjunto de dados é usualmente chamado de descoberta de conhecimento em bancos de dados - Knowledge Discovery in Databases (KDD). Esse processo, interativo e iterativo, incorpora conhecimento de domínio e interpretação de resultados, com ênfase na aplicação dos métodos de Mineração de Dados (MD) (Fayyad et al., 1996b). Pode-se definir a descoberta de conhecimento em bancos de dados como sendo o processo não trivial de identificação de padrões válidos, novos, potencialmente úteis e compreensíveis em grandes bancos de dados (Piatetsky-Shapiro e Frawley, 1991). O processo de descoberta de conhecimento em bancos de dados vem sendo aplicado em diversos domínios da Ciência e da Indústria. Algumas das aplicações descritas na literatura envolvem a automação de análise e catalogação de imagens de estrelas e galáxias (Fayyad et al., 1996a) e mapeamento de genes (Sevon et al., 2005). Diversas aplicações em outros domínios podem ser encontradas em Kantardzic e Zurada (2005); Rokach e Maimon (2008); Nisbet et al. (2009); Provost e Fawcett (2013).

O termo MD é comumente utilizado no lugar do termo KDD. Conceitualmente, porém, a MD é frequentemente considerada uma etapa no processo KDD, consistindo no uso de al- 
goritmos específicos, os quais encontram padrões sobre uma coleção de dados (Fayyad et al., 1996b). A MD é uma área interdisciplinar, possuindo intersecção com áreas como Aprendizado de Máquina, Reconhecimento de Padrões, Estatística e Bancos de Dados (Hand et al., 2001).

Os dados a serem analisados consistem em um conjunto ${ }^{1} \mathcal{X}$ de $N$ objetos (também chamados de tuplas, registros ou exemplos), i.e., $\mathcal{X}=\left\{\mathbf{x}_{n}\right\}_{n=1}^{N}$ sendo que cada $\mathbf{x}_{n}$ é um vetor de valores de um conjunto de $M$ atributos previsores (também chamados de características ou variáveis independentes) $A=\left\{A_{m}\right\}_{m=1}^{M}$. Usualmente os dados são representados no formato tabela atributo-valor - conforme ilustrado na Tabela 1.1.

\begin{tabular}{ccccc}
\multicolumn{1}{c}{} & $A_{1}$ & $A_{2}$ & $\ldots$ & $A_{M}$ \\
\cline { 2 - 5 } $\mathbf{x}_{1}$ & $x_{11}$ & $x_{12}$ & $\ldots$ & $x_{1 M}$ \\
$\mathbf{x}_{2}$ & $x_{21}$ & $x_{22}$ & $\ldots$ & $x_{2 M}$ \\
$\vdots$ & $\vdots$ & $\vdots$ & $\ddots$ & $\vdots$ \\
$\mathbf{x}_{N}$ & $x_{N 1}$ & $x_{N 2}$ & $\ldots$ & $x_{N M}$ \\
\cline { 2 - 5 } & & & &
\end{tabular}

Tabela 1.1: Base de dados representada no formato atributo-valor.

Existem, usualmente, duas abordagens para processar os dados durante o aprendizado. A primeira, denominada batch learning, utiliza informações de todos os dados disponíveis a cada iteração completa do algoritmo. Enquanto que na segunda, denominada de online learning, os dados são processados incrementalmente, um-a-um, ou em pequenos lotes a cada passo do algoritmo. Em algoritmos de aprendizado online, o conceito de iteração do algoritmo por vezes fica sem sentido. A escolha de qual abordagem adotar depende do problema em questão (Theodoridis e Koutroumbas, 2006). Em grandes bases de dados, o processamento online tem como atrativo não ser necessário carregar todos os dados em memória (Gama e Gaber, 2007; Shalev-Shwartz, 2012; Silva et al., 2013).

Os algoritmos utilizados na etapa de MD são usualmente categorizados pelo tipo de modelo de aprendizado que usam para encontrar padrões. As três principais categorias de aprendizado são: supervisionado, não supervisionado e semissupervisionado. Estas são descritas em mais detalhes na sequência.

\subsection{Aprendizado Supervisionado}

No aprendizado supervisionado, para cada objeto é fornecido também um rótulo da classe à qual o objeto pertence, i.e., cada objeto se torna uma tupla $\left\langle\mathbf{x}_{n}, c\right\rangle$, sendo $c$ a classe do objeto, e o problema de inferi-la para objetos em que a classe não é conhecida é comumente chamado de classificação ${ }^{2}$.

\footnotetext{
${ }^{1}$ Multi-conjuntos são usualmente permitidos.

${ }^{2} \mathrm{Em}$ aplicações em que o valor da classe é contínuo (e.g., determinar o valor do aluguel de um imóvel), o problema é comumente chamado de regressão.
} 
A classificação de dados é um processo que consiste de duas etapas (Han e Kamber, 2000): inicialmente, um modelo (classificador) é construído a partir de um conjunto de dados, usualmente chamado de conjunto de treinamento. Cada objeto do conjunto de treinamento é rotulado com uma classe $c_{j} \in C$, sendo $C=\left\{c_{1}, c_{2}, \ldots, c_{l}\right\}$ o conjunto das $l$ possíveis classes. Na segunda etapa, o modelo construído é utilizado para inferir a classe de objetos ainda não rotulados.

Existem diversas abordagens para a construção desses modelos, dentre as quais se destacam aquelas baseadas em: distâncias entre objetos (Aha et al., 1991), regra de Bayes (Bayes, 1763) e árvores de decisão (Quinlan, 1993). Para se escolher os modelos, usa-se um conceito muito difundido e conhecido como a Navalha de Occam (Occam's razor) (Elder e Pregibon, 1996) que sugere que, entre modelos com acurácia similar, o mais simples é preferível. Um potencial problema com modelos complexos é que eles tendem a possuir um menor poder de generalização - pois estão potencialmente super-ajustados aos dados de treinamento - o que os torna menos eficazes quando utilizados para fazer predições sobre novos dados. Tal problema é usualmente chamado de sobre-ajuste (overfitting). Para tentar obter melhores estimativas de acurácia - diminuindo o viés (bias) em relação aos dados de treinamento - são utilizadas técnicas de amostragem na construção do modelo. Dentre elas, a validação cruzada de $k$ pastas ( $k$-fold cross-validation) é amplamente utilizada (Witten e Frank, 2005), geralmente tomando $k=10$.

Algoritmos de classificação são muito utilizados em aplicações de diagnóstico médico (Kononenko, 2001) e identificação de SPAM (Zhang et al., 2004b). Para um estudo mais amplo sobre algoritmos de classificação, sugere-se consultar os trabalhos de Hand et al. (2001), Witten e Frank (2005), Bishop (2006), Aggarwal (2014) e Abou-Nasr et al. (2014).

\subsection{Aprendizado Não Supervisionado}

No aprendizado não supervisionado, apenas o conjunto de dados $(\mathcal{X})$ é fornecido e a principal tarefa, usualmente denominada de agrupamento, é formar uma partição que divida os dados em grupos de objetos similares (Jain e Dubes, 1988). O objetivo desta divisão é maximizar a homogeneidade entre os objetos de um mesmo grupo e, concomitantemente, maximizar a heterogeneidade entre objetos de grupos distintos. No entanto, o conceito de grupo é subjetivo; pessoas diferentes podem formar grupos diferentes com o mesmo conjunto de objetos.

Algoritmos de agrupamento de dados podem ser categorizados em dois tipos: hierárquicos e particionais (Xu e Wunsch, 2009). Algoritmos hierárquicos geram uma sequência de partições em que cada partição está aninhada ${ }^{3}$ na partição subsequente (Jain e Dubes, 1988). No caso de algoritmos particionais, os quais são o foco deste trabalho, apenas uma partição dos dados em $K$ grupos é gerada.

Pode-se classificar uma partição como sendo rígida (crisp) ou soft (Anderson et al., 2010).

\footnotetext{
${ }^{3}$ Uma partição $\mathcal{A}$ está aninhada em uma partição $\mathcal{B}$ se todo grupo de $\mathcal{A}$ é um subconjunto de um grupo de $\mathcal{B}$ (Jain e Dubes, 1988).
} 
Em uma partição rígida, cada objeto pertence a um único grupo. Partições desta categoria são frequentemente representadas de duas formas:

- Por uma matriz $\mathbf{U}_{K \times N}$, na qual $u_{k n}=1$ se o objeto $\mathbf{x}_{n}$ pertence ao $k$-ésimo grupo, e $u_{k n}=0$ caso contrário. Além disso, a seguinte restrição deve ser satisfeita:

$$
\sum_{k=1}^{K} u_{k n}=1, \forall n \in\{1, \ldots, N\}
$$

- Por uma coleção $\Pi=\left\{C_{k}\right\}_{k=1}^{K}$ de $K$ grupos disjuntos, sendo que $C_{k} \neq \emptyset, \forall k \in$ $\{1, \ldots, K\}$ e $\cup C_{k}=\mathcal{X}$.

No caso de partições soft, que são usualmente representadas pela matriz $\mathrm{U}$, a binarização em relação a um objeto pertencer a um grupo é relaxada, i.e., cada $u_{k n}$ pode ter um valor no intervalo $[0,1]$. Esta categoria de partições é útil em problemas nos quais existe sobreposição de grupos. Dentro desta categoria, são comuns três tipos de partições: probabilísticas, fuzzy e possibilísticas (Anderson et al., 2010). A principal diferença entre estes tipos está relacionada à interpretação dos valores em U. Em uma partição probabilística, $u_{k n}$ é interpretado como a probabilidade do objeto $\mathbf{x}_{n}$ ser oriundo do fenômeno (usualmente representado por uma distribuição de probabilidades) gerador do $k$-ésimo grupo. No caso de uma partição fuzzy, este valor refere-se ao grau de pertinência entre o objeto e o grupo. Finalmente, em partições possibilísticas, os valores $u_{k n}$ são interpretados como a possibilidade do objeto $\mathbf{x}_{n}$ ser proveniente do grupo $k$. É importante ressaltar que, destas, apenas as partições possibilísticas têm a restrição definida na Equação 1.1 relaxada. Nesta tese, tem-se como foco algoritmos que trabalham com partições rígidas e probabilísticas.

É importante ressaltar que a maioria dos algoritmos descritos na literatura assume que o número de grupos é fornecido pelo usuário (Kaufman e Rousseeuw, 1990; Milligan, 1996; Theodoridis e Koutroumbas, 2006; Aggarwal e Reddy, 2013; Celebi, 2014). Dessa forma, estes algoritmos se concentram em obter $K$ grupos de objetos semelhantes de acordo com algum critério pré-estabelecido. De acordo com Liu (1968), o número de partições rígidas possíveis para se agrupar $N$ objetos em $K$ grupos é dado por ${ }^{4}$ :

$$
\text { Num_Agrupamentos }(N, K)=\frac{1}{K !} \sum_{i=0}^{K}(-1)^{i}\left(\begin{array}{c}
K \\
i
\end{array}\right)(K-i)^{N} \text {. }
$$

Por exemplo, existem Num_Agrupamentos $(25,5)=2.436 .684 .974 .110 .751$ formas de agrupar 25 objetos em 5 grupos. Dessa maneira, pode-se perceber a complexidade de se agrupar corretamente uma base de dados de 25 objetos em 5 grupos. É necessário observar, ainda, que normalmente esse problema é considerado de tamanho bem reduzido quando comparado com aqueles encontrados em aplicações de MD (Hruschka, 2001). De fato, considerando o critério mais usual em algoritmos de agrupamento ${ }^{5}$, existem provas teóricas de que o problema é NP-

\footnotetext{
${ }^{4}$ Também conhecido como número de Stirling de tipo 2 (Graham et al., 1994).

${ }^{5}$ Minimização da soma da distância euclidiana ao quadrado entre objetos e centróides.
} 
difícil (Aloise et al., 2009). Por tal razão, nas últimas décadas tem sido estudado o uso de Algoritmos Evolutivos (AEs) (Eiben e Smith, 2003) em problemas de agrupamento de dados. Num sentido pragmático, AEs são metas-heurísticas efetivas em problemas NP-difíceis, capazes de obter boas soluções para tais problemas em tempo razoável (Hruschka et al., 2009). Uma das vantagens dos AEs em relação aos métodos de busca tradicionais é a sua maior robustez a ótimos locais; isso se deve principalmente ao fato de processarem um conjunto de soluções (população) ao invés de apenas uma solução (como ocorre em alguns métodos de busca tradicionais).

Técnicas de agrupamento de dados são amplamente utilizadas para processar dados de expressão gênica (bioinformática) (Yeung et al., 2003), categorizar documentos similares (mineração de texto) (Berry, 2003) e para encontrar perfis de consumidores (marketing) (Letrémy et al., 2007). Aplicações e desenvolvimentos recentes podem ser encontrados em Aggarwal e Reddy (2013) e Celebi (2014).

\subsection{Aprendizado Semissupervisionado}

Nas duas seções anteriores, foram abordados dois problemas de aprendizado que podem ser considerados extremos: o fornecimento de rótulos de classes para todos os objetos em $\mathcal{X}$ ou para nenhum dos objetos. No entanto, em diversas aplicações estão disponíveis informações adicionais ou conhecimento de domínio que podem auxiliar de forma parcial a encontrar soluções para o problema em questão (Basu et al., 2008), seja ele de natureza intrinsicamente supervisionada ou não-supervisionada. Estas informações suplementares podem ocorrer de diversas formas, e.g., na forma de rótulo de grupos ou classes para um subconjunto de objetos, de preferências do usuário sobre como objetos devem ser agrupados e de conhecimento sobre características dos grupos a serem formados. Neste contexto, técnicas de aprendizado semissupervisionado são úteis (Chapelle et al., 2010).

Dentre as linhas de pesquisa de aprendizado semissupervisionado, pode-se destacar (Goldberg, 2010):

- Classificação semissupervisionada: o conjunto de treinamento é formado por objetos rotulados e não rotulados. Usualmente, o número de objetos rotulados é significativamente menor que o número de objetos que não estão rotulados. O objetivo é treinar um classificador utilizando os dados rotulados e não rotulados, de forma que o classificador obtido seja melhor do que aquele treinado utilizando apenas os dados rotulados;

- Agrupamento de Dados com Restrições (ADR): além do conjunto de dados $\mathcal{X}$, algumas informações sobre a partição desejada são fornecidas. Essas informações podem ser fornecidas, por exemplo, por meio de alguns pares de objetos que devem pertencer ao mesmo grupo, bem como o número mínimo de objetos que um grupo deve ter. O objetivo é obter uma partição dos dados que seja melhor que a obtida de forma não supervisionada — sob o ponto de vista do usuário. 
Para um estudo amplo sobre classificação semissupervisionada, sugere-se consultar, por exemplo, Chapelle et al. (2010) e Goldberg (2010). A presente tese aborda, entre outros assuntos, Agrupamento de Dados com Restrições (ADR). Além disso, algoritmos para ADR podem ser utilizados, sob determinadas condições, para cenários de classificação semissupervisionada. Tais condições remetem à interpretação das restrições no contexto dos dados disponíveis, conforme será discutido ao longo da tese.

Algoritmos para aprendizado semissupervisionado têm sido aplicados com êxito em diversas áreas, tais como classificação de documentos (Nigam et al., 2000), detecção de pistas de estrada via GPS (Wagstaff et al., 2001), identificação de objetos em vídeos (Oyama e Tanaka, 2006) e preservação de privacidade em dados (Basu et al., 2008), e consequentemente, se constituem uma frente de pesquisa ativa atualmente (Wang et al., 2013; Li e Zemel, 2014; Acharya et al., 2014).

\subsection{Motivações e Hipóteses}

Modelos de mistura finitos são ferramentas de modelagem probabilística flexíveis e poderosas (McLachlan e Peel, 2000; Figueiredo e Jain, 2002). Conforme discutido por McLachlan e Peel (2000) e Jordan e Xu (1995), estes modelos fornecem um conveniente compromisso entre abordagens não-paramétricas e paramétricas. A flexibilidade não paramétrica provém do fato de que o número de componentes pode crescer conforme o necessário, enquanto que o fato de os componentes da mistura serem de forma paramétrica específica ${ }^{6}$ mantém a dimensão do espaço de busca em um tamanho razoável. Modelos de mistura finitos são utilizados em diversas áreas, como Biologia, Engenharia e Medicina (McLachlan e Peel, 2000). O uso de tais modelos para o agrupamento de dados permite tratar diversas questões, como o número de grupos, de maneira formal do ponto de vista estatístico (Fraley e Raftery, 1998, 2002; McLachlan e Peel, 2000).

Conforme será discutido no Capítulo 2, a definição de um modelo de mistura requer uma decisão sobre quais formas dos componentes são apropriadas para modelar os dados. É comum, na ausência de informação a priori, supor que os componentes do modelo são distribuições de probabilidades gaussianas (Jain e Dubes, 1988). Dentre as razões para tal, pode-se destacar o fato de que, por meio de um número suficiente de componentes gaussianos, pode-se aproximar quase qualquer função de densidade com acurácia arbitrária (Bishop, 2006).

Por tais razões, nesta tese tem-se como material de estudo os modelos de mistura finitos, nos quais os componentes são distribuições de probabilidades gaussianas. Estes modelos costumam ser denominados Gaussian Mixture Models (GMMs). Em aplicações de agrupamento de dados, tanto não supervisionado quanto semissupervisionado, o uso destes modelos apresenta alguns desafios. Especificamente, a estimação de parâmetros de um GMM pode ser vista como um problema de otimização que tem como característica um grande número de mínimos locais.

\footnotetext{
${ }^{6}$ Realizações deste tipo de modelo serão abordadas no Capítulo 2.
} 
Embora esse seja um cenário típico para aplicação de AEs, o uso destes considerando modelos gerais, com o menor número de premissas possíveis, ainda é pouco explorado. Além disso, os poucos trabalhos nesta linha de pesquisa não fazem uso da extensa literatura existente sobre o assunto no contexto de algoritmos de busca locais. Levando isso em consideração, a primeira hipótese desta tese é definida como:

Hipótese 1: Um AE desenvolvido considerando estratégias bem conhecidas da literatura de algoritmos de busca local é capaz de estimar os parâmetros de um GMM, tendo como conhecimento a priori apenas os dados e sem nenhuma imposição estrutural sob o modelo, de forma eficiente e competitiva com abordagens já existentes para a estimação de parâmetros.

Considerando cenários de ADR, duas linhas de pesquisa são pouco exploradas na literatura. A primeira consiste em algoritmos capazes de tratar tanto os dados quanto as restrições de forma online, e.g., dados processados sob demanda. Este tipo de algoritmo é especialmente relevante em aplicações envolvendo grandes bases de dados. Baseando-se nesta lacuna da literatura, a segunda hipótese desta tese é definida como:

Hipótese 2: Um algoritmo de ADR que processa os dados de forma online é capaz de obter acurácia comparável com algoritmos da literatura de ADR que processam os dados em lotes (batches).

Ao incorporar determinados tipos de restrições em um GMM, gera-se uma complexidade adicional à estimação dos seus parâmetros. Especificamente, o uso de restrições sobre pares de objetos, especialmente se dois objetos devem ou não estar no mesmo grupo ${ }^{7}$, cria uma dependência entre rótulos de objetos distintos. Tal dependência torna o modelo complexo e, usualmente, requer aproximações para que o mesmo possa ser usado na prática. Além disso, por muitas vezes estas restrições são obtidas de rótulos de classe ou sem conhecimento sobre a disposição espacial dos dados no espaço de atributos, conforme será discutido no Capítulo 4. Como uma classe não necessariamente corresponde a um grupo, em ambas as situações as restrições podem não ter nenhuma concordância com a distância entre objetos, critério pelo qual a maioria dos algoritmos de agrupamento se baseia para formar grupos. Por esta razão, diversos algoritmos da literatura ficam presos a soluções sub-ótimas quando tentam incorporar tais restrições em suas partições dos dados. Uma forma de se evitar tal problema é considerar que as restrições referem-se a classes, e que tais classes podem ser formadas por mais de um grupo. Embora existam trabalhos na literatura nessa linha de pesquisa (Zhao e Miller, 2005; Raghuram et al., 2014), os mesmos dependem de um parâmetro que define um compromisso entre atender restrições e a qualidade da solução, que por sua vez é de difícil ajuste por parte do usuário. Levando em consideração todos esses aspectos, formula-se a terceira hipótese desta tese:

\footnotetext{
${ }^{7}$ A descrição desse tipo de restrição é realizado no Capítulo 4.
} 
Hipótese 3: É possível desenvolver algoritmos para ADR com as seguintes propriedades: (i) capacidade de lidar com situações de mais de um grupo por classe, mesmo que recebendo como supervisão restrições entre pares de objetos; (ii) ausência de parâmetros críticos de compromisso entre qualidade de soluções e rigidez de restrições; (iii) desobrigação de que o usuário defina o número de grupos por classe a priori; (iv) eficiência computacional.

Considerando o exposto, esta tese tem como objetivo contribuir com a literatura tanto de aprendizado não supervisionado quanto semissupervisionado. Os algoritmos propostos, bem como os experimentos realizados para validar as hipóteses aqui levantadas, constituem contribuições relevantes para diversas áreas, tais como: aprendizado de máquina, algoritmos evolutivos e MD. Boa parte do material apresentado nessa tese foi publicada durante o trabalho de doutoramento (Covões e Hruschka, 2011; Sestaro et al., 2012; Covões e Hruschka, 2013; Covões et al., 2013c,a,b).

\subsection{Organização da Tese}

O restante desta tese está organizado da seguinte forma:

Capítulo 2 - Modelos de Mistura de Gaussianas: Apresenta a formalização do conceito de Gaussian Mixture Models (GMMs) e estratégias descritas na literatura para evitar mínimos locais na estimação de seus parâmetros. Este capítulo também serve para introduzir parte significativa da notação matemática adotada.

Capítulo 3 - Algoritmos Evolutivos para Modelos de Mistura de Gaussianas Não Supervisionados: Descreve, à luz de trabalhos existentes na literatura, o algoritmo Evolutionary Split \& Merge Algorithm for Expectation Maximization (ESM-EM), que combina as duas estratégias revisadas no Capítulo 2 para estimar de forma eficiente os parâmetros de um GMM. Adicionalmente, diversas variantes deste algoritmo são comparadas em 35 bases de dados. A melhor variante deste algoritmo obteve resultados competitivos com o algoritmo estado-da-arte baseado em um AE para a estimação de parâmetros de um GMM. As contribuições deste capítulo encontram-se em revisão em periódico internacional.

Capítulo 4 - Agrupamento de Dados com Restrições: Neste capítulo, é apresentado um estudo sobre ADR. São também apresentados conceitos e terminologias referentes a ADR utilizadas neste trabalho. Finalmente, é realizado um levantamento bibliográfico sobre diversos trabalhos desta área do conhecimento.

Capítulo 5 - Aprendizado Competitivo para Agrupamento de Dados com Restrições: Este capítulo apresenta dois algoritmos desenvolvidos para ADR e que são capazes de processar os dados de forma online. Mais especificamente, o algoritmo Constrained Rival 
Penalized Competitive Learning consiste de uma adaptação de um algoritmo bem conhecido na literatura de aprendizado competitivo para o cenário de ADR. Enquanto que o algoritmo Linear Constrained Vector Quantization Error foi desenvolvido a partir do algoritmo de ADR Linear Constrained Vector Quantization Error, cuja eficiência e eficácia foram avaliadas em um estudo comparativo empírico previamente (Covões et al., 2013c). Os algoritmos foram avaliados em 8 bases de dados e apresentaram resultados competitivos com o algoritmo LCVQE, com menor custo computacional. As análises deste capítulo foram publicadas em periódico internacional (Covões et al., 2013a).

Capítulo 6 - Modelando Múltiplos Grupos por Classe: O algoritmo MCCK, que foi projetado para identificar múltiplos grupos por classe, via $K$-means, é descrito neste capítulo. Este algoritmo de ADR é capaz de estimar o número de grupos por classe a partir de um conjunto de restrições ML sob certas condições. Experimentos em 8 bases de dados e comparações com dois algoritmos bem conhecidos da literatura de aprendizado semissupervisionado (Basu et al., 2002) são reportados. Os resultados obtidos demonstram que estimar o número de grupos por classe pode conduzir a resultados significativos em diversas bases de benchmark. O conteúdo deste capítulo foi publicado em Sestaro et al. (2012).

\section{Capítulo 7 - Algoritmo Evolutivo para ADR para Modelos de Mistura de Gaussianas que} Utiliza Restrições: Neste capítulo, é introduzido o algoritmo FIECE-EM, que pode ser visto como uma generalização do algoritmo MCCK para GMMs com matrizes de covariância irrestritas. Este algoritmo foi comparado em 8 bases de dados com o algoritmo estado-da-arte com premissas similares (Raghuram et al., 2014). Os resultados obtidos pelo FIECE-EM demonstram que o algoritmo pode ser útil em diversas aplicações, sendo competitivo com o algoritmo que representa o estado-da-arte. Pretende-se publicar as análises descritas neste capítulo em um artigo de periódico internacional. Atualmente, este artigo encontra-se em produção.

Capítulo 8 - Conclusões e Trabalhos Futuros: Este capítulo sumariza as principais conclusões originadas desta tese, além de apresentar possíveis linhas de pesquisa para trabalhos futuros. 


\section{CAPÍTULO}

\section{Modelos de Mistura de Gaussianas}

\subsection{Considerações Iniciais}

Neste capítulo, são apresentados, brevemente, os principais conceitos relacionados aos modelos de mistura de gaussianas (Gaussian Mixture Models (GMMs)) relevantes para esta tese. A literatura sobre GMM é extensa, em especial em alternativas para estimar os parâmetros do modelo. Para um estudo mais amplo sobre o assunto, sugere-se ao leitor os trabalhos de McLachlan e Krishnan (1997) e McLachlan e Peel (2000).

Um GMM é definido por uma função de densidade de probabilidade - $f()-$. tal que:

$$
f\left(\mathbf{x}_{n} \mid \Psi\right)=\sum_{k=1}^{K} \pi_{k} \mathcal{N}\left(\mathbf{x}_{n} \mid \boldsymbol{\mu}_{k}, \boldsymbol{\Sigma}_{k}\right)
$$

na qual $\left\{\pi_{k}\right\}_{k=1}^{K}$ são os coeficientes de mistura, também chamados de probabilidades a priori, sujeitos a: $0 \leq \pi_{k} \leq 1, \sum_{k=1}^{K} \pi_{k}=1, K$ é o número de componentes da mistura ${ }^{1}$, $\mathcal{N}$ é a distribuição de probabilidade gaussiana multivariada definida na Equação 2.2 e $\Psi=$ $\left\{\left\{\pi_{k}\right\}_{k=1}^{K},\left\{\boldsymbol{\mu}_{k}\right\}_{k=1}^{K},\left\{\boldsymbol{\Sigma}_{k}\right\}_{k=1}^{K}\right\}$ é o conjunto de parâmetros do modelo (McLachlan e Peel, 2000). Neste caso, os parâmetros são os coeficientes de mistura e os parâmetros de cada distribuição de probabilidade gaussiana (médias $\left(\boldsymbol{\mu}_{k}\right)$ e matrizes de covariância $\left(\Sigma_{k}\right)$ ).

$$
\mathcal{N}\left(\mathbf{x}_{n} \mid \boldsymbol{\mu}_{k}, \boldsymbol{\Sigma}_{k}\right)=\frac{1}{(2 \pi)^{\frac{M}{2}}\left|\boldsymbol{\Sigma}_{k}\right|^{\frac{1}{2}}} \exp \left\{-\frac{1}{2}\left(\mathbf{x}_{n}-\boldsymbol{\mu}_{k}\right)^{T} \boldsymbol{\Sigma}_{k}^{-1}\left(\mathbf{x}_{n}-\boldsymbol{\mu}_{k}\right)\right\},
$$

Embora seja possível utilizar outras distribuições de probabilidades no modelo de mistura, existem diversos motivos para se considerar distribuições gaussianas. Dentre eles, o uso de um

\footnotetext{
${ }^{1}$ Nesta tese, focamos no conceito de modelos de mistura finitos. Caso o leitor tenha interesse em modelos de mistura infinitos, sugere-se o trabalho de Rasmussen (2000).
} 
número suficiente de gaussianas, com o ajuste de seus parâmetros, permite aproximar um grande número de funções de densidade contínua com uma acurácia arbitrária (Bishop, 2006). Por tal razão, GMMs são amplamente utilizados em diversas aplicações, tais como reconhecimento de orador (Reynolds e Rose, 1995) e de dígitos manuscritos (Chen et al., 2011), segmentação de imagens (Zhang et al., 2003) e análise de imagens de ressonância magnética do cérebro (Tohka et al., 2007).

\subsection{Estimação de Parâmetros}

Uma tarefa comum em diversas aplicações envolve estimar, para um conjunto de dados observados $-\mathcal{X}=\left\{\mathbf{x}_{n}\right\}_{n=1}^{N}, \mathbf{x}_{n} \in \mathbb{R}^{M}$ ), em que as $N$ amostras são independentes e identicamente distribuídas — os parâmetros do GMM gerador dos mesmos. Nesta tese, é considerado o uso do algoritmo Expectation Maximization (EM) para a estimação dos parâmetros do GMM.

O algoritmo EM (Dempster et al., 1977) é uma abordagem para a computação iterativa de estimativas de máxima verossimilhança - Maximum Likelihood Estimate (MLE) — útil para uma variedade de problemas com dados incompletos (McLachlan e Krishnan, 1997). De forma intuitiva, o EM associa um problema com dados incompletos a um com dados completos cuja estimação da verossimilhança seja mais tratável (Bishop, 2006). A literatura sobre EM e GMM é rica, e sugere-se ao leitor interessado os trabalho de Dempster et al. (1977); McLachlan e Krishnan (1997); Dasgupta (1999); McLachlan e Peel (2000); Bishop (2006). Caso o leitor já esteja familiarizado com o EM no contexto de GMMs, não é necessária a leitura desta seção. Por conveniência, é utilizada a mesma notação do popular livro de Bishop (2006).

Para a aplicação do EM, inicialmente define-se o conjunto completo de dados. Neste caso, o conjunto completo é formado pelo conjunto de dados observados $\left(\mathcal{X}=\left\{\mathbf{x}_{n}\right\}_{n=1}^{N}, \mathbf{x}_{n} \in \mathbb{R}^{M}\right)$, e um conjunto correspondente de vetores indicadores $\left(\mathcal{Z}=\left\{\mathbf{z}_{n}\right\}_{n=1}^{N}, \mathbf{z}_{n} \in\{0,1\}^{K}\right)$, que indicam a qual dos $K$ componentes a $n$-ésima amostra pertence ${ }^{2}$.

A função de verossimilhança define a probabilidade de serem observados os dados completos condicionada aos parâmetros, i.e., $p(\mathcal{X}, \mathcal{Z} \mid \Psi)$. No entanto, os valores em $\mathcal{Z}$ são desconhecidos e, por tal razão, é necessário computar o valor esperado dos vetores indicadores, dado por (Bishop, 2006):

$$
\gamma\left(z_{n k}\right)=\mathbb{E}\left[z_{n k}\right]=\frac{\pi_{k} \mathcal{N}\left(\mathbf{x}_{n} \mid \boldsymbol{\mu}_{k}, \boldsymbol{\Sigma}_{k}\right)}{\sum_{j=1}^{K} \pi_{j} \mathcal{N}\left(\mathbf{x}_{n} \mid \boldsymbol{\mu}_{j}, \boldsymbol{\Sigma}_{j}\right)},
$$

na qual $\mathbb{E}$ denota o operador de esperança. Esta quantidade pode ser vista como a responsabilidade do $k$-ésimo componente em explicar a observação $\mathbf{x}_{n}$ (Bishop, 2006). Considerando estas responsabilidades, pode-se obter o valor esperado da função logarítmica de verossimilhança ${ }^{3}$

\footnotetext{
${ }^{2} \mathbf{z}_{n}$ utiliza uma representação 1-para- $K$.

${ }^{3}$ Esta função é usualmente utilizada no lugar da função de verossimilhança devido a duas propriedades: $\log (a \dot{b})=\log (a)+\log (b)$, que torna a computação mais eficiente; a função $\log ($.$) é monotonicamente cres-$ cente em relação ao seu argumento. Portanto, maximizar a função de verossimilhança é equivalente a maximizar a
} 
(Bishop, 2006):

$$
L L K(\mathcal{X} \mid \Psi)=\mathbb{E}_{\mathcal{Z}}[\ln p(\mathcal{X}, \mathcal{Z} \mid \Psi)]=\sum_{n=1}^{N} \sum_{k=1}^{K} \gamma\left(z_{n k}\right)\left\{\ln \pi_{k}+\ln \mathcal{N}\left(\mathbf{x}_{n} \mid \boldsymbol{\mu}_{k}, \boldsymbol{\Sigma}_{k}\right)\right\}
$$

O algoritmo EM para este modelo pode ser então resumido da seguinte forma. No primeiro passo, chamado de passo E (Expectation), avaliam-se as responsabilidades esperadas $\left(\gamma\left(z_{n k}\right)\right)$ de cada objeto para cada componente. É importante observar que, para tal, são necessários os parâmetros do modelo $(\Psi)$. No caso da primeira iteração, não há nenhuma estimativa para os seus valores. Neste caso, diversas abordagens podem ser utilizadas para fornecer valores iniciais para o algoritmo. Uma das alternativas é utilizar o algoritmo $\mathrm{K}$-means ${ }^{4}$ (McLachlan e Peel, 2000). No segundo passo, denominado de passo M (Maximization), os parâmetros do modelo são ajustados de forma a maximizar a função de verossimilhança. As atualizações dos parâmetros referentes a cada componente $k$ são realizadas de acordo com:

$$
\begin{aligned}
\pi_{k}= & \frac{\sum_{n=1}^{N} \gamma\left(z_{n k}\right)}{N}, \\
\boldsymbol{\mu}_{k}= & \frac{\sum_{n=1}^{N} \gamma\left(z_{n k}\right) \mathbf{x}_{n}}{\sum_{n=1}^{N} \gamma\left(z_{n k}\right)} \\
\boldsymbol{\Sigma}_{k}= & \frac{\sum_{n=1}^{N} \gamma\left(z_{n k}\right)\left(\mathbf{x}_{n}-\boldsymbol{\mu}_{k}\right)\left(\mathbf{x}_{n}-\boldsymbol{\mu}_{k}\right)^{T}}{\sum_{n=1}^{N} \gamma\left(z_{n k}\right)}
\end{aligned}
$$

Os passos E e M são repetidos iterativamente até a convergência, dada usualmente por uma mudança pequena no valor da função logarítmica de verossimilhança. É conhecido que o algoritmo EM converge para um ótimo local da função de verossimilhança. Porém, também já foi constatado que o algoritmo EM demora a convergir em diversas aplicações (McLachlan e Krishnan, 1997).

Como a convergência para um ótimo global não é garantida, é comum repetir diversas vezes o algoritmo, a partir de inicializações diferentes, e selecionar a melhor delas, considerando-se a função de verossimilhança. Além disso, em diversas aplicações o número de componentes não é conhecido a priori. Neste caso, pode-se executar o algoritmo para diferentes números de componentes e selecionar, dentre elas, qual a melhor de acordo com alguma função de avaliação

${ }^{4}$ Embora o algoritmo $K$-means também seja sensível à inicialização do seus parâmetros, por ser eficiente computacionalmente, pode-se executá-lo diversas vezes e selecionar a melhor solução de acordo com algum critério. 
que permita avaliar modelos de diferentes complexidades ${ }^{5}$. Nesta tese, essa estratégia para encontrar o melhor modelo, quando o número de componentes é desconhecido, é referenciada por Ordered Multiple Runs of Expectation Maximization (OMR-EM). O Algoritmo 1 sumariza esta estratégia, sendo $s$ uma função de avaliação a ser minimizada (um exemplo de função é apresentada na Seção 3.2) e $S_{C}$ é um critério de parada para o EM, e.g., número máximo de iterações. Na prática, $n_{p}$ é usualmente definido de maneira ad-hoc ou considerando os recursos computacionais disponíveis. Assumindo que $n_{p}$ é proporcional a $K_{\max }$ (número máximo de grupos), o custo computacional do procedimento é $O\left(N \cdot K_{\max }^{2} \cdot M^{3}\right)^{6}$.

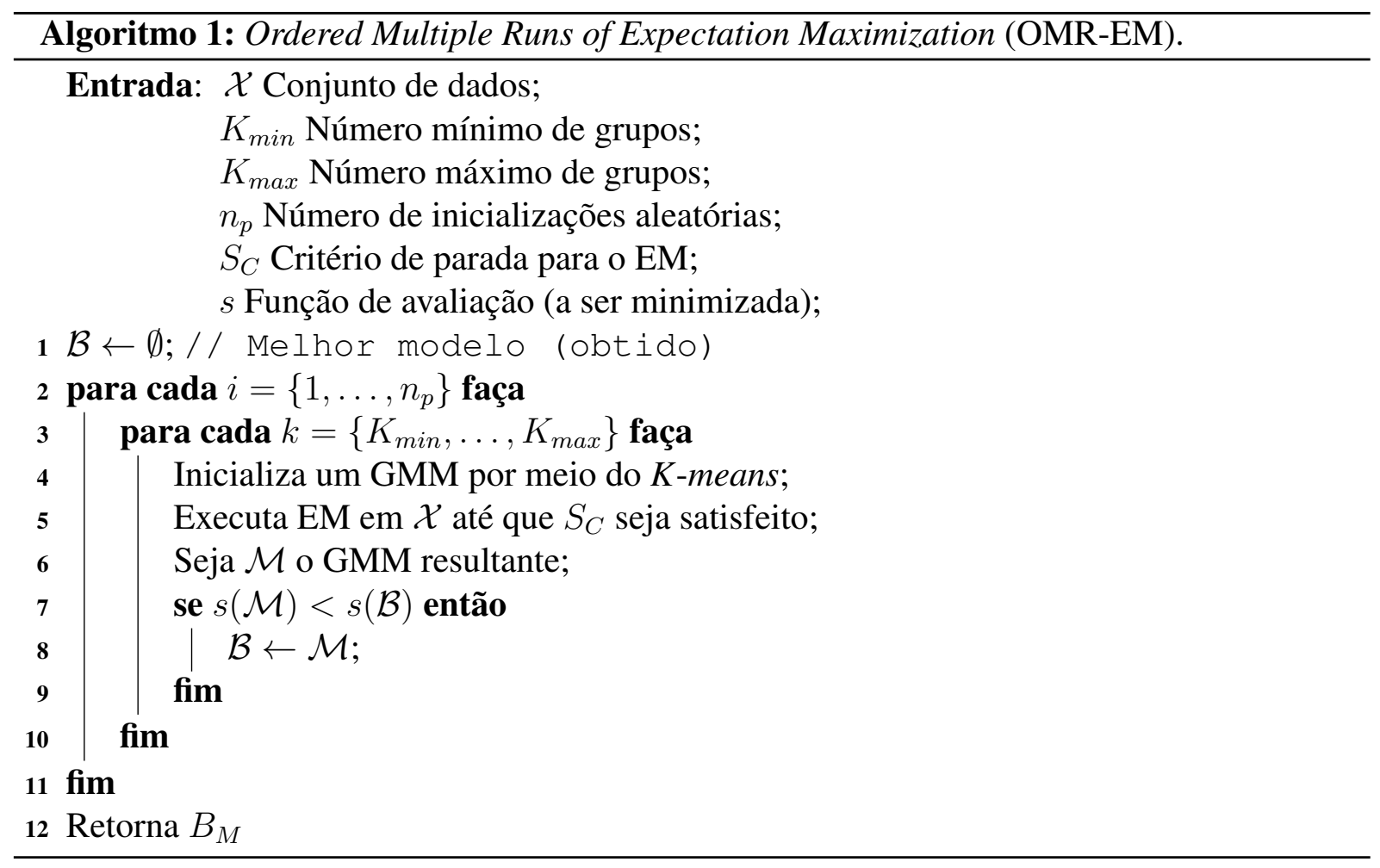

Na procura de se ter métodos que explorem o espaço de busca de forma mais eficiente, considerando modificações na solução atual de forma a se obter uma nova que melhor se ajuste aos dados, diversas abordagens foram propostas (McLachlan e Krishnan, 1997; Ueda e Nakano, 1998; McLachlan e Peel, 2000; Ueda et al., 2000; Zhang et al., 2003; Figueiredo e Jain, 2002; Verbeek et al., 2003; Pernkopf e Bouchaffra, 2005; Constantinopoulos e Likas, 2007). Duas abordagens são de interesse nesta tese. A primeira consiste na divisão e união de componentes - Split and Merge (SM) - que foi inicialmente apresentada por Ueda et al. (2000). A segunda utiliza algoritmos evolutivos para trabalhar com mais de uma solução (modelo) simultaneamente (Pernkopf e Bouchaffra, 2005).

O uso do EM para aprender GMM pode ser visto, do ponto de vista de agrupamento de dados, como o particionamento dos dados em $K$ grupos de forma probabilística. Portanto, cada

\footnotetext{
${ }^{5} \mathrm{~A}$ função de verossimilhança não pode ser usada para avaliar modelos de diferentes complexidades por ser monotonicamente crescente em relação ao número de grupos (Figueiredo e Jain, 2002; Bishop, 2006).

${ }^{6}$ Uma análise detalhada, no contexto do K-means, pode ser encontrada em Naldi et al. (2011).
} 
componente representa um grupo. De fato, caso se considere cada objeto como pertencendo ao grupo de maior probabilidade e matrizes de covariância diagonais e proporcionais à matriz identidade, o EM para GMM se reduz ao algoritmo K-means (Bishop, 2006). O uso de operações para dividir e unir grupos em algoritmos de agrupamento de dados não é novo, e.g., Liu e Tsai (1989) e Chaudhuri et al. (1992). Além disso, com o avanço da pesquisa em AEs para agrupamento de dados, para os quais é comum a implementação de tais operações por operadores de mutação (Hruschka et al., 2009), este tipo de operação é de grande interesse para a área de agrupamento de dados no contexto geral. No que concerne a esta tese, a revisão de procedimentos de união e divisão de componentes busca servir de fundamentação para o desenvolvimento de um AE de agrupamento de dados baseado no EM para GMM que utilize tais operações no processo de busca. Por tal razão, é apresentada uma revisão sobre os procedimentos adotados por algoritmos de união e divisão de componentes na próxima seção. Na Seção 2.4.2, são apresentados brevemente os principais conceitos de AEs, bem como seus respectivos métodos relacionados a esta tese.

\subsection{Procedimentos para União e Divisão de Componentes em Gaussian Mixture Models}

De acordo com Ueda et al. (2000), uma das dificuldades encontradas por variantes do EM para evitar ótimos locais é devido à incapacidade de um componente ser movido de uma região densa para outra, passando por uma região esparsa, sem produzir menores valores de verossimilhança. A ideia básica de algoritmos Split and Merge (SM) é unir dois componentes em uma região densa e dividir um componente em uma região esparsa. Dessa forma, mínimos locais podem ser evitados e o espaço de busca de parâmetros pode ser melhor explorado.

Nas Seções 2.3.1 e 2.3.2, são descritos os procedimentos para união e divisão de componentes, respectivamente. Além das operações para a definição dos novos componentes, também são descritos diversos critérios comumente aplicados para definir quais componentes devem ser unidos/divididos.

\subsubsection{União de Componentes}

A união de componentes tem como intuito reduzir a complexidade do GMM, combinando componentes que modelam a mesma região densa. A forma de avaliar essa sobreposição comumente utilizada é a correlação entre as responsabilidades dos componentes (Ueda et al., 2000; Zhang et al., 2003; Ma e He, 2005; Blekas e Lagaris, 2007). Sejam $k_{1}$ e $k_{2}$ os índices de dois componentes, o critério é definido como:

$$
U_{\text {correlation }}\left(k_{1}, k_{2} \mid \Psi\right)=\frac{\mathbf{u}_{k_{1}}^{T} \mathbf{u}_{k_{2}}}{\left\|\mathbf{u}_{k_{1}}\right\|\left\|\mathbf{u}_{k_{2}}\right\|},
$$


na qual $\mathbf{u}_{k_{1}}=\left(\gamma\left(z_{1 k_{1}}\right), \ldots, \gamma\left(z_{N k_{1}}\right)\right)^{T} \in \mathbb{R}^{N}$. O par de componentes com maior valor absoluto de correlação é o melhor candidato para a união. Em Ueda et al. (2000) e Blekas e Lagaris (2007), o critério é utilizado sem a normalização, o que torna o critério polarizado em relação a componentes com muitos objetos. Uma variação deste critério, utilizando o coeficiente de correlação de Pearson no lugar da medida do coseno, é adotado por Wang et al. (2004).

Uma alternativa à medida de correlação é o critério de distância mínima entre distribuições (Symmetric Kullback Leibler) (Blekas e Lagaris, 2007):

$$
\begin{aligned}
U_{\text {distance }}\left(k_{1}, k_{2} \mid \Psi\right) & =\sum_{n=1}^{N} \tilde{\mathcal{N}}\left(\mathbf{x}_{n} \mid \boldsymbol{\mu}_{k_{1}}, \boldsymbol{\Sigma}_{k_{1}}\right) \log \frac{\tilde{\mathcal{N}}\left(\mathbf{x}_{n} \mid \boldsymbol{\mu}_{k_{1}}, \boldsymbol{\Sigma}_{k_{1}}\right)}{\tilde{\mathcal{N}}\left(\mathbf{x}_{n} \mid \boldsymbol{\mu}_{k_{2}}, \boldsymbol{\Sigma}_{k_{2}}\right)} \\
& +\sum_{n=1}^{N} \tilde{\mathcal{N}}\left(\mathbf{x}_{n} \mid \boldsymbol{\mu}_{k_{2}}, \boldsymbol{\Sigma}_{k_{2}}\right) \log \frac{\tilde{\mathcal{N}}\left(\mathbf{x}_{n} \mid \boldsymbol{\mu}_{k_{2}}, \boldsymbol{\Sigma}_{k_{2}}\right)}{\tilde{\mathcal{N}}\left(\mathbf{x}_{n} \mid \boldsymbol{\mu}_{k_{1}}, \boldsymbol{\Sigma}_{k_{1}}\right)} . \\
\tilde{\mathcal{N}}\left(\mathbf{x}_{n} \mid \boldsymbol{\mu}_{k}, \boldsymbol{\Sigma}_{k}\right) & =\frac{\mathcal{N}\left(\mathbf{x}_{n} \mid \boldsymbol{\mu}_{k}, \boldsymbol{\Sigma}_{k}\right)}{\sum_{n^{\prime}=1}^{N} \mathcal{N}\left(\mathbf{x}_{n^{\prime}} \mid \boldsymbol{\mu}_{k}, \boldsymbol{\Sigma}_{k}\right)},
\end{aligned}
$$

A desvantagem dessa medida, em relação a correlação, é que ela considera as componentes isoladamente, sem considerar o contexto do GMM.

Li e Ma (2008) adotam uma abordagem diferente, baseada na sobreposição de componentes, para qualificar um par de componentes a serem unidos. Considere $\phi\left(\mathbf{x}_{n}, k\right)=\gamma\left(z_{n k}\right)(1-$ $\left.\gamma\left(z_{n k}\right)\right), \Omega_{k}^{\epsilon}=\left\{\mathbf{x}_{n} \in \mathcal{X} \mid \gamma\left(z_{n k}\right)>0.5 \wedge \phi\left(\mathbf{x}_{n} \mid k\right) \geq \epsilon\right\}$, e dist $\left(k_{1}, k_{2}\right)$ a distância de Mahalanobis entre os dois componentes, a medida de sobreposição é definida como:

$$
U_{\text {overlap }}\left(k_{1}, k_{2} \mid \Psi, \epsilon\right)=\frac{\left[\sum_{\mathbf{x}_{n} \in \Omega_{k_{2}}^{\epsilon}} \phi\left(\mathbf{x}_{n}, k_{1}\right)\right]\left[\sum_{\mathbf{x}_{n} \in \Omega_{k_{1}}^{\epsilon}} \phi\left(\mathbf{x}_{n}, k_{2}\right)\right]}{\left|\Omega_{k_{2}}^{\epsilon}\right|\left|\Omega_{k_{1}}^{\epsilon}\right| \operatorname{dist}\left(k_{1}, k_{2}\right)} .
$$

A escolha de um valor para $\epsilon$ é difícil para o usuário, já que não há uma heurística para definilo, sendo necessária uma verificação de vários valores para avaliar qual melhor se adapta aos dados utilizados. Além disso, o cálculo da distância de Mahalanobis entre dois componentes é computacionalmente custoso. Em particular, quando utilizada a medida proposta por Anderson e Bahadur (1962), o custo computacional estimado é de $O\left(M^{3}\right)$.

Após a definição de quais componentes devem ser unidos, é necessário definir como os parâmetros dos mesmos devem ser combinados. No trabalho de Ueda et al. (2000), é proposto um método de união de componentes baseado em uma combinação linear ponderada para determinar os parâmetros de um novo componente $k_{3}$ obtido por meio da união dos componentes $k_{1} \mathrm{e}$ $k_{2}$ :

$$
\begin{aligned}
\pi_{k_{3}} & =\pi_{k_{1}}+\pi_{k_{2}} \\
\boldsymbol{\mu}_{k_{3}} & =\frac{\pi_{k_{1}} \boldsymbol{\mu}_{k_{1}}+\pi_{k_{2}} \boldsymbol{\mu}_{k_{2}}}{\pi_{k_{1}}+\pi_{k_{2}}}
\end{aligned}
$$




$$
\Sigma_{k_{3}}=\frac{\pi_{k_{1}} \Sigma_{k_{1}}+\pi_{k_{2}} \Sigma_{k_{2}}}{\pi_{k_{1}}+\pi_{k_{2}}}
$$

Este método também foi aplicado em Wang et al. (2004) e Blekas e Lagaris (2007). Porém, tal método foi criticado por Zhang et al. (2003) devido à atualização da média não ser influenciada pela matriz de covariância, e vice-versa. Como a média e a matriz de covariância são, respectivamente, o primeiro e o segundo momento da distribuição, tratá-las de forma independente não é adequado. Por tal razão, Zhang et al. (2003) derivaram uma expressão adequada para a obtenção da matriz de covariância, sendo a média fornecida pela Equação (2.12):

$$
\boldsymbol{\Sigma}_{k_{3}}=\frac{1}{\pi_{k_{3}}}\left(\pi_{k_{1}} \boldsymbol{\Sigma}_{k_{1}}+\pi_{k_{1}} \boldsymbol{\mu}_{k_{1}} \boldsymbol{\mu}_{k_{1}}^{T}+\pi_{k_{2}} \boldsymbol{\Sigma}_{k_{2}}+\pi_{k_{2}} \boldsymbol{\mu}_{k_{2}} \boldsymbol{\mu}_{k_{2}}^{T}\right)-\boldsymbol{\mu}_{k_{3}} \boldsymbol{\mu}_{k_{3}}^{T}
$$

Esta solução também é adotada por Zhang et al. (2004a), Ma e He (2005) e Li e Li (2009).

\subsubsection{Divisão de Componentes}

Em contrapartida à união de componentes, a divisão de componentes atua modificando componentes que modelam regiões de dados esparsas, dando origem a novos componentes em suas regiões mais densas. O critério mais usado para identificar qual componente deve ser dividido é o Local Kullback Divergence (LKD) (Ueda et al., 2000; Zhang et al., 2003; Wang et al., 2004; Zhang et al., 2004a; Blekas e Lagaris, 2007). Considerando a avaliação do $k$-ésimo componente, o critério é definido como:

$$
\begin{aligned}
D_{L K D}(k \mid \boldsymbol{\Psi}) & =\sum_{n=1}^{N} f_{k}\left(\mathbf{x}_{n} \mid \boldsymbol{\Psi}\right) \log \frac{f_{k}\left(\mathbf{x}_{n} \mid \boldsymbol{\Psi}\right)}{\tilde{\mathcal{N}}\left(\mathbf{x}_{n} \mid \boldsymbol{\mu}_{k}, \mathbf{\Sigma}_{k}\right)}, \\
\tilde{\mathcal{N}}\left(\mathbf{x}_{n} \mid \boldsymbol{\mu}_{k}, \boldsymbol{\Sigma}_{k}\right) & =\frac{\mathcal{N}\left(\mathbf{x}_{n} \mid \boldsymbol{\mu}_{k}, \boldsymbol{\Sigma}_{k}\right)}{\sum_{n^{\prime}=1}^{N} \mathcal{N}\left(\mathbf{x}_{n^{\prime}} \mid \boldsymbol{\mu}_{k}, \mathbf{\Sigma}_{k}\right)}, \\
f_{k}\left(\mathbf{x}_{n} \mid \Psi\right) & =\frac{\sum_{n^{\prime}=1}^{N} \delta\left(\mathbf{x}_{n^{\prime}}-\mathbf{x}_{n}\right) \gamma\left(z_{n^{\prime} k}\right)}{\sum_{n^{\prime}=1}^{N} \gamma\left(z_{n^{\prime} k}\right)} \\
\delta(\mathbf{v}) & =\left\{\begin{array}{cc}
1, & \text { para } \mathbf{v}=\mathbf{0}, \\
0, & \text { caso contrário }
\end{array}\right.
\end{aligned}
$$

sendo que $f_{k}\left(\mathbf{x}_{n} \mid \Psi\right)$ é a densidade local dos dados próximos ao $k$-ésimo componente e $\delta(\mathbf{v})$ é conhecida como função Kronecker (Bracewell, 1999). A Equação (2.15) define a distância entre duas distribuições: a densidade local dos dados $f_{k}\left(\mathbf{x}_{n} \mid \Psi\right)$ na vizinhança da $k$-ésima distribuição gaussiana e a sua densidade considerando o modelo especificado pelos parâmetros atuais (Ueda et al., 2000). A maximização de $D_{L K D}$ indica uma estimativa ruim da densidade local. Portanto, o componente que maximiza este critério é o mais indicado para ser dividido. Outros 
critérios utilizados na literatura envolvem outras medidas de entropia ( $\mathrm{Li} \mathrm{e} \mathrm{Li}, 2009$ ) e testes de normalidade (Ma e He, 2005).

Para a divisão de um componente $k$ em dois componentes $k^{\prime}$ e $k^{\prime \prime}$, Ueda et al. (2000) propuseram que os parâmetros dos novos componentes fossem ajustados de acordo com:

$$
\begin{aligned}
& \pi_{k^{\prime}}=\frac{\pi_{k}}{2} \quad, \quad \pi_{k^{\prime \prime}}=\frac{\pi_{k}}{2} \\
& \boldsymbol{\mu}_{k^{\prime}}=\boldsymbol{\mu}_{k}+\epsilon, \boldsymbol{\mu}_{k^{\prime \prime}}=\boldsymbol{\mu}_{k}+\epsilon^{\prime} \\
& \boldsymbol{\Sigma}_{k^{\prime}}=\left|\boldsymbol{\Sigma}_{k}\right|^{\frac{1}{M}} \mathbf{I}, \quad \boldsymbol{\Sigma}_{k^{\prime \prime}}=\left|\boldsymbol{\Sigma}_{k}\right|^{\frac{1}{M}} \mathbf{I}
\end{aligned}
$$

sendo $\epsilon$ e $\epsilon^{\prime}$ vetores de perturbação aleatórios pequenos (i.e., $\left.\|\epsilon\| \ll\left\|\boldsymbol{\mu}_{k}\right\|\right),\left|\Sigma_{k}\right|$ é o determinante da matriz de covariância $\Sigma_{k}$ e I é a matriz identidade. É possível verificar duas limitações neste método. A primeira é que a matriz de covariância de cada novo componente é isotrópica, o que geometricamente indica que o grupo é formado por uma hiperesfera, independente dos dados envolvidos no processo. A segunda limitação é que, assim como no método de união de componentes, as matrizes de covariância e as médias são ajustadas independentemente uma da outra, ignorando assim sua relação como primeiro e segundo momento da distribuição, conforme observado por Zhang et al. (2003). Apesar disso, este método também é utilizado por Wang et al. (2004). Uma variação é descrita em Zhang et al. (2004a), na qual as médias e os coeficientes de mistura dos novos componentes são ajustados para valores aleatórios e a hiperesfera é ajustada de acordo com a maior diferença observada nos dados.

Em Blekas e Lagaris (2007), os coeficientes de mistura e a matriz de covariância são apenas divididos igualmente entre os dois componentes. A média de cada componente novo é movida considerando o maior autovalor e autovetor da matriz de covariância, que são relacionados com o eixo principal de maior variância, do componente original. Mais especificamente:

$$
\begin{aligned}
\pi_{k^{\prime}} & =\frac{\pi_{k}}{2}, & \pi_{k^{\prime \prime}} & =\frac{\pi_{k}}{2} \\
\boldsymbol{\Sigma}_{k^{\prime}} & =\frac{\boldsymbol{\Sigma}_{k}}{2}, & \boldsymbol{\Sigma}_{k^{\prime \prime}} & =\frac{\boldsymbol{\Sigma}_{k}}{2}, \\
\boldsymbol{\mu}_{k^{\prime}} & =\boldsymbol{\mu}_{k}+\frac{\sqrt{\lambda_{\max }}}{2} \mathbf{v}_{\max }, & \boldsymbol{\mu}_{k^{\prime \prime}} & =\boldsymbol{\mu}_{k}-\frac{\sqrt{\lambda_{\max }}}{2} \mathbf{v}_{\max }
\end{aligned}
$$

na qual $\lambda_{\max }$ e $\mathbf{v}_{\max }$ são o maior autovalor de $\Sigma_{k}$ e seu autovetor correspondente, respectivamente. É possível observar que, assim como o método proposto por Ueda et al. (2000), este também não relaciona a atualização da média com a atualização da matriz de covariância.

Zhang et al. (2003) mostram que, diferentemente da união de componentes, não é possível encontrar uma solução fechada para a divisão de componentes mantendo a relação entre a média e a matriz de covariância, porque o número de equações é menor que o número de variáveis. Apesar disso, os autores propuseram dois métodos para a divisão de componentes. O primeiro 
é baseado na Singular Value Decomposition (SVD) (Golub e Van Loan, 1996). Inicialmente, a matriz de covariância $\Sigma_{k}$ é decomposta em três matrizes $\mathbf{U S V}^{T}$ tal que $\mathbf{S}$ é a matriz de valores singulares em ordem decrescente, e $\mathbf{U}$ e $\mathbf{V}$ são duas matrizes ortogonais (Golub e Van Loan, 1996). Define-se então a matriz $\mathbf{A}_{k}=\mathbf{U} \sqrt{\mathbf{S}}$ (Li e Li, 2009). De acordo com Zhang et al. (2003), o problema de determinar $\boldsymbol{\Sigma}_{k^{\prime}}$ e $\boldsymbol{\Sigma}_{k^{\prime \prime}}$ dada $\boldsymbol{\Sigma}_{k}$ é modificado para o problema de determinar $\mathbf{A}_{k^{\prime}}$ e $\mathbf{A}_{k^{\prime \prime}}$, dado $\mathbf{A}_{k}$. Considerando $\mathbf{a}_{i}^{(k)}$ a $i$-ésima coluna da matriz $\mathbf{A}_{k}$, os parâmetros são ajustados da seguinte forma (maiores detalhes em Zhang et al. (2003)):

$$
\begin{aligned}
\pi_{k^{\prime}} & =\pi_{k} \alpha \\
\pi_{k^{\prime \prime}} & =\pi_{k}(1-\alpha) \\
\boldsymbol{\mu}_{k^{\prime}} & =\boldsymbol{\mu}_{k}-\sqrt{\frac{\pi_{k^{\prime \prime}}}{\pi_{k^{\prime}}}} u \mathbf{a}_{1}^{(k)}, \\
\boldsymbol{\mu}_{k^{\prime \prime}} & =\boldsymbol{\mu}_{k}+\sqrt{\frac{\pi_{k^{\prime}}}{\pi_{k^{\prime \prime}}}} u \mathbf{a}_{1}^{(k)}, \\
\boldsymbol{\Sigma}_{k^{\prime}} & =\frac{\pi_{k^{\prime \prime}}}{\pi_{k^{\prime}}} \boldsymbol{\Sigma}_{k}+\left(\beta-\beta u^{2}-1\right) \frac{\pi_{k}}{\pi_{k^{\prime}}} \mathbf{a}_{1}^{(k)}\left(\mathbf{a}_{1}^{(k)}\right)^{T}+\mathbf{a}_{1}^{(k)}\left(\mathbf{a}_{1}^{(k)}\right)^{T} \\
\boldsymbol{\Sigma}_{k^{\prime \prime}} & =\frac{\pi_{k^{\prime}}}{\pi_{k^{\prime \prime}}} \boldsymbol{\Sigma}_{k}+\left(\beta u^{2}-\beta-u^{2}\right) \frac{\pi_{k}}{\pi_{k^{\prime \prime}}} \mathbf{a}_{1}^{(k)}\left(\mathbf{a}_{1}^{(k)}\right)^{T}+\mathbf{a}_{1}^{(k)}\left(\mathbf{a}_{1}^{(k)}\right)^{T}
\end{aligned}
$$

nas quais $\alpha, u, \beta \in(0,1)$ são parâmetros definidos pelo usuário. Embora qualquer um dos outros vetores $\left(\mathbf{a}_{i}^{(k)}\right.$ ) possa ser usado no lugar de $\mathbf{a}_{1}^{(k)}$, o uso deste é justificado por corresponder ao primeiro eixo principal (Zhang et al., 2003). Este método também é aplicado por Ma e $\mathrm{He}$ (2005); Li e Li (2009). O segundo método é baseado na decomposição de Cholesky (Golub e Van Loan, 1996), e apesar de similar ao primeiro, os próprios autores (Zhang et al., 2003) consideram que o método baseado em SVD faz melhor uso da informação dos autovalores e autovetores.

Todos os trabalhos baseados na união e divisão de componentes citados nesta seção utilizam tais operações em conjunto com algoritmos que efetuam a busca pelos parâmetros de forma gulosa e considerando apenas uma solução por vez. Nesta tese, o uso de tais operações é combinado com algoritmos evolutivos, que são descritos em detalhes na próxima seção. Essa combinação permite uma busca mais computacionalmente eficiente e com menos chances de ficar presa em mínimos locais.

\subsection{Algoritmos Evolutivos}

Algoritmos Evolutivos (AEs) consistem em algoritmos de busca estocásticos, baseados em abstrações do processo de evolução darwiniano (Freitas, 2002). Tais algoritmos se baseiam no processamento de um conjunto de soluções (usualmente chamadas de indivíduos) de tal forma a resolver um determinado problema, usualmente de otimização matemática. Cada solução é ava- 
liada por uma função objetivo (fitness), que mede sua qualidade ou adequabilidade. Levando-se em conta a adequabilidade das soluções, procedimentos de seleção baseados na seleção natural (indivíduos melhor adaptados têm maior chance de reproduzir e sobreviver) e a aplicação de operadores baseados na genética - como cruzamento (crossover) e mutação (mutation) evoluem a população de indivíduos. O processo é repetido até que um determinado critério de parada seja satisfeito.

Os principais componentes de um AE são (Eiben e Smith, 2003):

- Representação: define a forma como as soluções candidatas do problema serão representadas para o algoritmo evolutivo. Esta representação interna ao algoritmo evolutivo é denominada genótipo e define matematicamente o espaço de busca;

- Função de adequabilidade (fitness) ou função objetivo: mensura a qualidade de qualquer solução candidata pertencente ao espaço de busca;

- População: representa um conjunto de soluções candidatas;

- Mecanismo de seleção de pais: considerando a adequabilidade dos indivíduos, são selecionados quais indivíduos serão usados para gerar novas soluções candidatas;

- Operadores: criam novas soluções candidatas baseando-se nas soluções da população atual. São divididos em duas categorias: mutação e cruzamento (crossover). Um operador de mutação realiza uma modificação em uma solução. Um operador de cruzamento une a informação de duas soluções em uma ou duas soluções novas. É usual que cada operador tenha uma determinada probabilidade de ocorrer em cada geração;

- Mecanismo de seleção de sobreviventes: similar ao mecanismo de seleção de pais, define entre as soluções candidatas quais serão mantidas na próxima população.

Existem alguns subtipos de AEs (Freitas, 2002; Eiben e Smith, 2003), dentre os quais se destacam: estratégias evolutivas; programação evolutiva; algoritmos genéticos; programação genética. As principais diferenças entre eles encontram-se na forma como os indivíduos são representados e na aplicação dos operadores.

Num sentido mais pragmático, AEs são metas-heurísticas efetivas em problemas NP-Difíceis, capazes de obter boas soluções para tais problemas em tempo razoável (Hruschka et al., 2009). Uma das vantagens dos AEs em relação a métodos de busca tradicionais é a sua maior robustez a ótimos locais; isso se deve principalmente ao fato de processarem um conjunto de soluções (população) ao invés de apenas uma solução (como ocorre em alguns métodos de busca tradicionais), além de permitirem trabalhar com intensificação e diversificação. Uma revisão profunda sobre AEs está além do escopo deste trabalho. Para tal, sugere-se ao leitor os trabalhos de Bäck et al. (2000a,b), Eiben e Smith (2003) e Ashlock (2006). 
Diversos trabalhos na literatura empregam AEs em problemas de mineração de dados (Freitas, 2002). No contexto de problemas de agrupamento de dados não supervisionado, diversos AEs foram propostos, especialmente, nas últimas duas décadas. Uma revisão bibliográfica pode ser encontrada em Hruschka et al. (2009) e em Naldi (2011).

Conforme mencionado na Seção 2.2, GMMs podem ser vistos, do ponto de vista de agrupamento de dados, como o particionamento dos dados em $K$ grupos de forma probabilística. Portanto, cada componente da mistura representa um grupo. Diferentemente do particionamento rígido dos dados, neste modelo cada objeto $\left(\mathbf{x}_{n}\right)$ passa a pertencer a um grupo $\left(C_{k}\right)$ com certa probabilidade denotada por $\gamma\left(z_{n k}\right)$. Em outras palavras, uma partição probabilística dos dados $\mathbf{U}=\left(\mathbf{u}_{1}, \ldots, \mathbf{u}_{K}\right)$, na qual cada coluna é igual às responsabilidades de um componente, i.e., $\mathbf{u}_{k}=\left(\gamma\left(z_{1 k}\right), \ldots, \gamma\left(z_{N k}\right)\right), k \in\{1, \ldots, K\}$. Neste sentido, diversos AEs desenvolvidos para agrupamento de dados podem ser vistos como estimadores de parâmetros de GMMs. Por tal razão, nesta tese foram desenvolvidos algoritmos que combinam abordagens que apresentaram eficácia em ambas as áreas.

Para facilitar a descrição e caracterização dos AEs para agrupamento de dados, neste trabalho é utilizada a taxonomia definida por Falkenauer (1998) e estendida por Hruschka et al. (2009):

- Número de grupos fixo ou variável: indica se o algoritmo necessita que o usuário forneça o número de grupos a serem obtidos ou se o número de grupos é estimado automaticamente, usualmente por meio de critérios de validação relativos (Jain e Dubes, 1988).

- Operadores orientados e não orientados a grupos: orientação a grupos define se o operador foi especialmente concebido para processar uma partição de dados via operações como copiar, dividir, unir e eliminar grupos.

- Operadores de cruzamento sensíveis e insensíveis ao contexto: o operador deve ser orientado a grupos e, considerando o cruzamento de dois indivíduos possivelmente diferentes que representam a mesma solução (partição), um operador de cruzamento sensível ao contexto deve gerar como filho a mesma solução.

- Operadores guiados ou não guiados: indica se o operador utiliza informação sobre a qualidade dos grupos, da partição ou de seu desempenho em operações realizadas anteriormente para definir se e como será aplicado, ou em qual grupo irá atuar.

- Tipo de representação: as três representações mais comuns são: baseada em medóides ${ }^{7}$, em rótulos e em descrição de grupos. Na primeira representação, cada indivíduo é representado pelo medóide de cada grupo da partição. No caso da representação baseada em rótulos, o indivíduo é representado pelo rótulo do grupo para o qual cada objeto foi

\footnotetext{
${ }^{7}$ Medóide é o objeto que tem a menor distância média em relação aos outros objetos de seu grupo (Xu e Wunsch, 2009).
} 
atribuído. Finalmente, na representação baseada em descrição de grupos, o indivíduo é representado pelos parâmetros que especificam os grupos.

Naldi et al. (2011) realizaram diversos experimentos empíricos que indicam que AEs bem projetados podem ser eficientes computacionalmente para otimizar partições obtidas por meio do popular algoritmo $K$-means, em especial quando comparado com estratégias de amostragem comumente utilizadas na prática. Levando isto em consideração, e ainda lembrando que o algoritmo K-means é uma especialização do algoritmo EM para GMM (Bishop, 2006), este trabalho parte da hipótese de que o uso de um AE em conjunto com variantes do EM para GMM pode apresentar resultados melhores do que outros algoritmos reportados na literatura, os quais se baseiam, em sua grande maioria, em diversas execuções de algoritmos baseados em busca local.

Nas Seções 2.4.1 e 2.4.2, são descritos alguns algoritmos baseados no K-means e no EM para GMM. Tais algoritmos são usados tanto como fundamentos para o desenvolvimento de um AE que é descrito no Capítulo 3, quanto como baseline de comparação. Porém, antes de detalhar os algoritmos, é interessante definir algumas notações. Em particular, $\mathcal{P}^{(t)}$ refere-se à população na $t$-ésima iteração, $P_{\text {mutation }}$ é uma função que calcula a probabilidade de mutação de seus argumentos, $p_{m}$ é a probabilidade de mutação de um indivíduo e $p_{c}$ é a probabilidade de cruzamento de um indivíduo. Um indivíduo é denotado por $\mathcal{G}$, independente da codificação de seu genótipo.

\subsubsection{Algoritmos Evolutivos Baseados em $K$-means}

Diversos trabalhos abordam o uso de algoritmos evolutivos para otimizar partições obtidas pelo $K$-means. Sugere-se ao leitor interessado em uma revisão da área os trabalhos de Hruschka et al. (2009); Chang et al. (2009); Niknam e Amiri (2010) e Cai et al. (2011). Na próxima seção, é descrito o AE utilizado como ponto de partida para o desenvolvimento de um AE baseado no EM para GMM.

\subsubsection{Fast Evolutionary Algorithm for Clustering}

Em Alves et al. (2006), foi introduzido o algoritmo evolutivo para agrupamento de dados não supervisionado, denominado Fast Evolutionary Algorithm for Clustering (F-EAC), que apresentou melhores resultados em relação ao seu antecessor Evolutionary Algorithm For Clustering (EAC) (Hruschka et al., 2004, 2006). O algoritmo foi desenvolvido com o objetivo de superar duas limitações básicas do algoritmo K-means: necessidade de definir o número de grupos e convergência para ótimos locais. Em Naldi et al. (2011), foram apresentados experimentos, considerando-se cenários nos quais não se conhece o número de grupos a priori, que mostram que o F-EAC apresenta resultados tão bons quanto algumas estratégias de amostragem usualmente utilizadas em conjunto com o $K$-means, mas com menor custo computacional. 
No F-EAC, cada partição dos dados é codificada em um genótipo utilizando codificação inteira baseada em rótulos (Hruschka et al., 2009). Por exemplo, o genótipo [1,1,2,2,3,1] representa a partição $C_{1}=\{1,2,6\}, C_{2}=\{3,4\}$ e $C_{3}=\{5\}$. Conforme mencionado em Hruschka et al. (2009), essa codificação tem a deficiência de ser redundante, já que a mesma partição pode ser codificada de $K$ ! formas diferentes.

A função objetivo utilizada no algoritmo é a Silhueta Simplificada (SS) (Hruschka et al., 2006). Antes de apresentar a SS, será apresentada a silhueta originalmente proposta por Kaufman e Rousseeuw (1990). Considerando o objeto $\mathbf{x}_{i}$ pertencendo ao grupo $C_{j}$, a distância média do objeto $\mathrm{x}_{i}$ em relação a todos os outros objetos pertencentes ao grupo $C_{j}$ será chamada de $a(i)$. Levando em consideração o grupo $C_{r}$, a distância média do objeto $\mathbf{x}_{i}$ em relação a todos os outros objetos pertencentes ao grupo $C_{r}$ pode ser chamada de $d\left(i, C_{r}\right)$. Após calcular $d\left(i, C_{r}\right)$ para todos os grupos $C_{r} \neq C_{j}$, o menor valor de $d\left(i, C_{r}\right)$ (aqui chamado de $b(i)$ ) é escolhido. Mais precisamente, $b(i)=\min d\left(i, C_{r}\right), C_{r} \neq C_{j}$. Este valor representa a distância entre o objeto $\mathbf{x}_{i}$ e o seu grupo vizinho. Feitas estas considerações, a silhueta $s(i)$ é dada por:

$$
s(i)=\frac{b(i)-a(i)}{\max \{a(i), b(i)\}} .
$$

Considerando que a partição pode ser gerada de forma aleatória, pode-se verificar que $-1 \leq$ $s(i) \leq 1$. Logo, quanto maior o valor de $s(i)$, melhor terá sido a atribuição do objeto $\mathbf{x}_{i}$ para um dado grupo. Se $s(i)=0$, então não está claro se o objeto $\mathbf{x}_{i}$ deveria ser agrupado com o grupo atual ou com o seu grupo vizinho mais próximo (Everitt, 2001). Finalmente, se o grupo $C_{j}$ possui um único elemento, então $s(i)$ não é definida e uma escolha adequada é considerar $s(i)=0$ (Kaufman e Rousseeuw, 1990). A média dos valores de $s(i)$ para todos os objetos, i.e., $\frac{1}{N} \sum_{i=1}^{N} s(i)$, é usada como o critério de avaliação de uma partição, sendo o melhor agrupamento determinado quando o seu valor é maximizado.

A silhueta proposta por Kaufman e Rousseeuw (1990) depende do cálculo de todas as distâncias entre os (pares de) objetos, levando a um custo computacional de $O\left(N^{2}\right)$, o qual normalmente é muito alto para aplicações de MD. Para suprir essa limitação, a Silhueta Simplificada (SS) (Hruschka et al., 2006) pode ser utilizada. A SS é baseada no cálculo das distâncias entre os objetos e os centróides dos grupos. Mais especificamente, o termo $a(i)$ se torna a distância entre o objeto $\mathbf{x}_{i}$ e o centróide do seu grupo $\left(\mathbf{c}_{j}\right)$. Similarmente, ao invés de calcular $d\left(i, C_{r}\right)$ como a distância média do objeto $\mathbf{x}_{i}$ em relação a todos os objetos de $C_{r}, C_{r} \neq C_{j}$, são calculadas as distâncias entre o objeto $\mathbf{x}_{i}$ e o centróide do grupo $C_{r}\left(\mathbf{c}_{r}\right)$. Essas modificações reduzem o custo computacional de $O\left(N^{2}\right)$ para $O(N \cdot K)$.

Para realizar a busca no espaço de soluções candidatas, são utilizados dois operadores de mutação. Para a aplicação de ambos os operadores, é necessária uma medida que indique a qualidade de cada grupo. Em outras palavras, estes operadores são guiados. O algoritmo F-EAC baseia-se na premissa de que quanto melhor o grupo, menor deve ser sua probabilidade de sofrer mutação. Dessa forma, bons grupos tendem a permanecer durante as gerações, enquanto que 
grupos ruins tendem a sofrer modificações (Naldi et al., 2011). Para que haja uma sinergia entre a aplicação dos operadores e a função objetivo, é necessário que essa função possa ser decomposta para cada grupo, ou seja, a qualidade mensurada para determinar a probabilidade de aplicação dos operadores é baseada na contribuição do grupo para a qualidade da partição como um todo. No caso da SS, essa decomposição é trivial já que a medida da qualidade de uma partição é a média da silhueta de todos os objetos. Assim sendo, a qualidade de um grupo pode ser mensurada pela média da silhueta dos objetos nele contidos.

A probabilidade de um grupo $C_{k}$ ser escolhido para mutação é descrita pela Equação (2.32), na qual rank é uma função que retorna a posição do valor da qualidade do grupo em um ranking considerando os valores de qualidade de todos os grupos. Neste caso, o grupo com maior qualidade de acordo com a SS encontra-se na posição 1 do ranking. Esta normalização baseada em ranking permite evitar que um grupo com uma qualidade muito inferior aos demais, de acordo com a SS, domine as probabilidades (Bäck et al., 2000a).

$$
p_{m}\left(C_{k}\right)=\frac{\operatorname{rank}\left(1-\sum_{\mathbf{x}_{n} \in C_{k}} \frac{s(n)}{\left|C_{k}\right|}\right)}{\sum_{j=1}^{K} \operatorname{rank}\left(1-\sum_{\mathbf{x}_{n} \in C_{j}} \frac{s(n)}{\left|C_{j}\right|}\right)}
$$

O primeiro operador (MO1) realiza a eliminação de grupos alocando os objetos dos grupos que serão eliminados nos grupos mais próximos. Este operador é descrito no Algoritmo 2. O segundo operador (MO2) divide um grupo em dois e é descrito no Algoritmo 3. Considerando a taxonomia previamente apresentada, ambos os operadores são guiados e orientados a grupos.

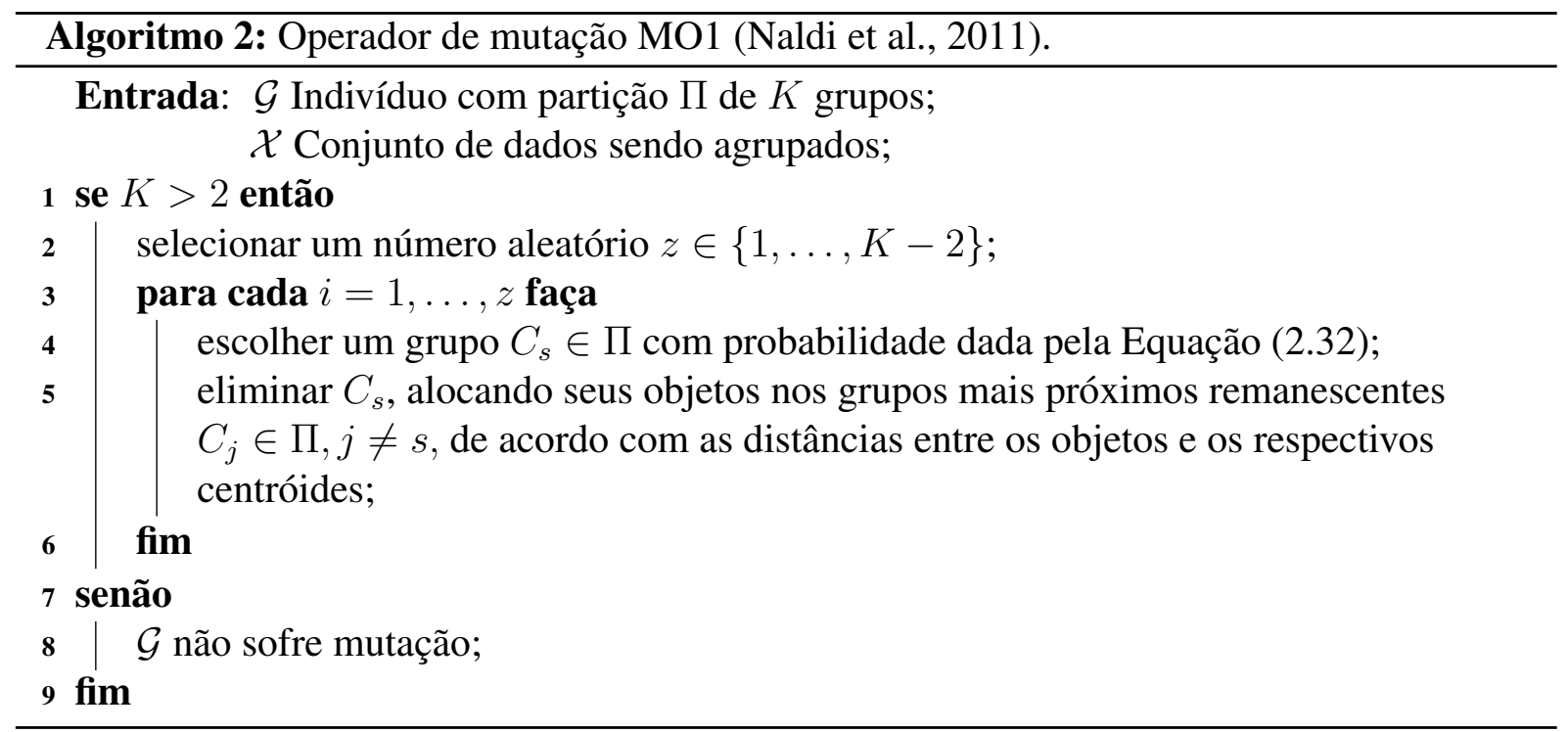

Para ajustar as taxas de aplicação dos dois operadores, duas estratégias já foram investigadas (Alves et al., 2006; Horta e Campello, 2009). A primeira (Alves et al., 2006), utilizada na primeira versão do F-EAC, ajusta as probabilidades de acordo com o desempenho dos operadores em toda a população, enquanto a segunda (Horta e Campello, 2009) avalia o impacto da aplicação dos operadores sobre um determinado indivíduo. Resultados empíricos em Horta e 


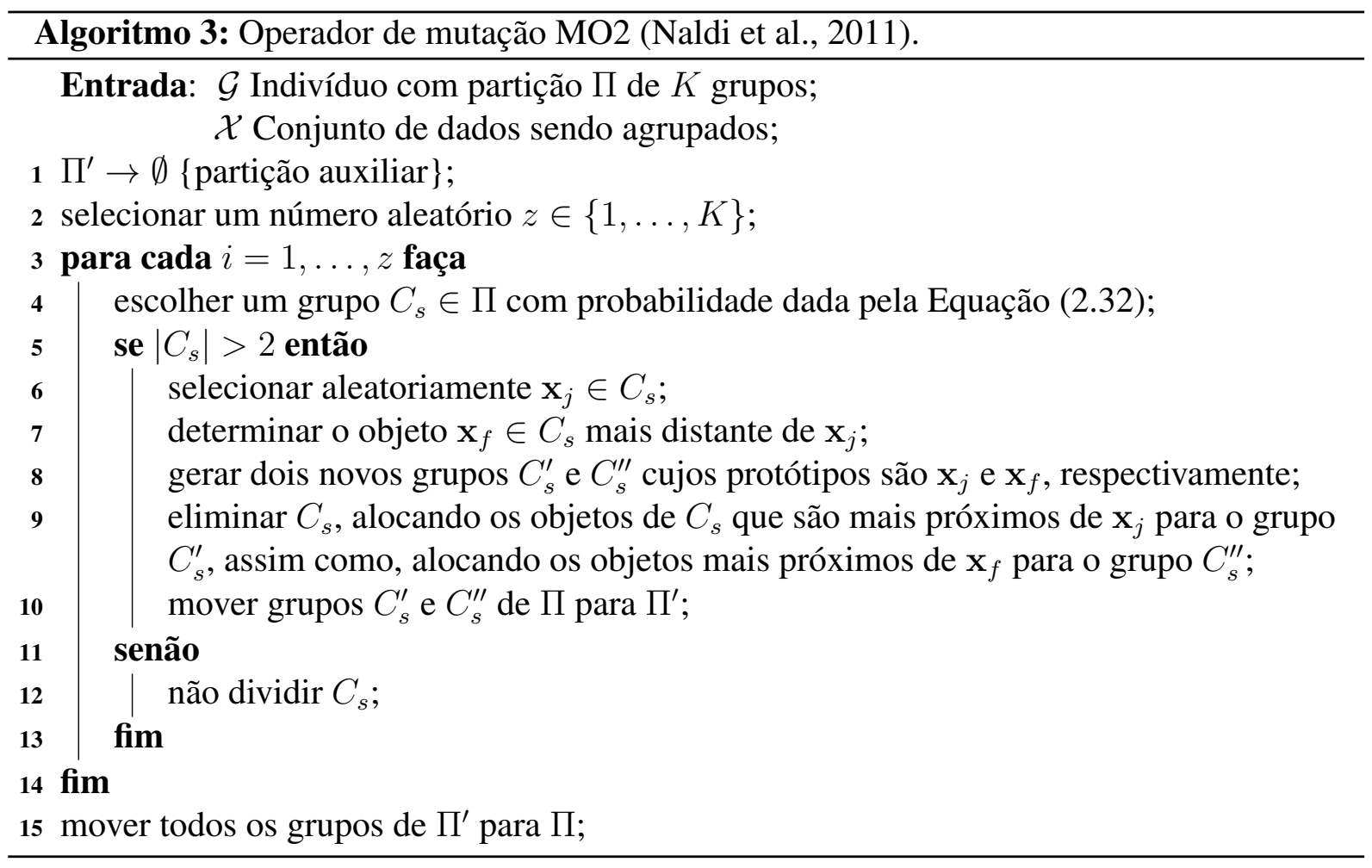

Campello (2009) sugerem que a segunda estratégia apresenta resultados melhores ou equivalentes à primeira. Por tal razão, neste trabalho apenas esta estratégia é descrita e sugere-se ao leitor interessado em maiores detalhes sobre a primeira estratégia consultar Alves et al. (2006) e Naldi (2011). Considere que um indivíduo teve um aumento na sua adequabilidade de uma geração $t$ para a geração $t+1$. Neste caso, o mesmo operador aplicado anteriormente é utilizado. Caso o indivíduo tenha obtido um decréscimo na sua adequabilidade, o operador a ser aplicado é substituído. Finalmente, se nenhum operador foi aplicado no indivíduo, um dos dois operadores (MO1 e MO2) é escolhido aleatoriamente, com igual probabilidade (Horta e Campello, 2009).

Para a seleção dos indivíduos para a próxima geração, dois operadores são utilizados: elitismo e seleção por ranking (Bäck et al., 2000a). O primeiro garante que o melhor indivíduo seja mantido de uma geração para outra, enquanto que o segundo permite que os indivíduos sejam selecionados com probabilidades proporcionais às qualidades das soluções que representam. Na seleção é utilizado o procedimento de normalização por ranking e o algoritmo da roleta, assim como feito para o cálculo da probabilidade de um grupo sofrer mutação. No entanto, neste caso, o pior indivíduo da população tem posição 1 no ranking. Todos os indivíduos selecionados utilizando ranking passam por um dos operadores de mutação, de acordo com o procedimento supramencionado.

Para fazer o refinamento das partições obtidas, o algoritmo $K$-means é aplicado em cada um dos indivíduos. Conforme mencionado em Naldi et al. (2011), poucas iterações do K-means são suficientes para realizar este procedimento de busca local. Portanto, é interessante utilizar um número máximo de iterações para o $K$-means. Isso é esperado pelo fato do F-EAC favorecer 
o refinamento acumulativo das partições, mantendo boas partições para gerações subsequentes. $\mathrm{O}$ algoritmo F-EAC pode ser sumarizado pelo Algoritmo 4.

O F-EAC já foi utilizado como ponto de partida para variantes em diversos contextos de agrupamento de dados, tais como em: agrupamento rígido relacional (Horta e Campello, 2009), agrupamento fuzzy relacional (Horta et al., 2011) e, além disso, numa variante de seu predecessor (EAC) para imputação de valores (Silva e Hruschka, 2009, 2013).

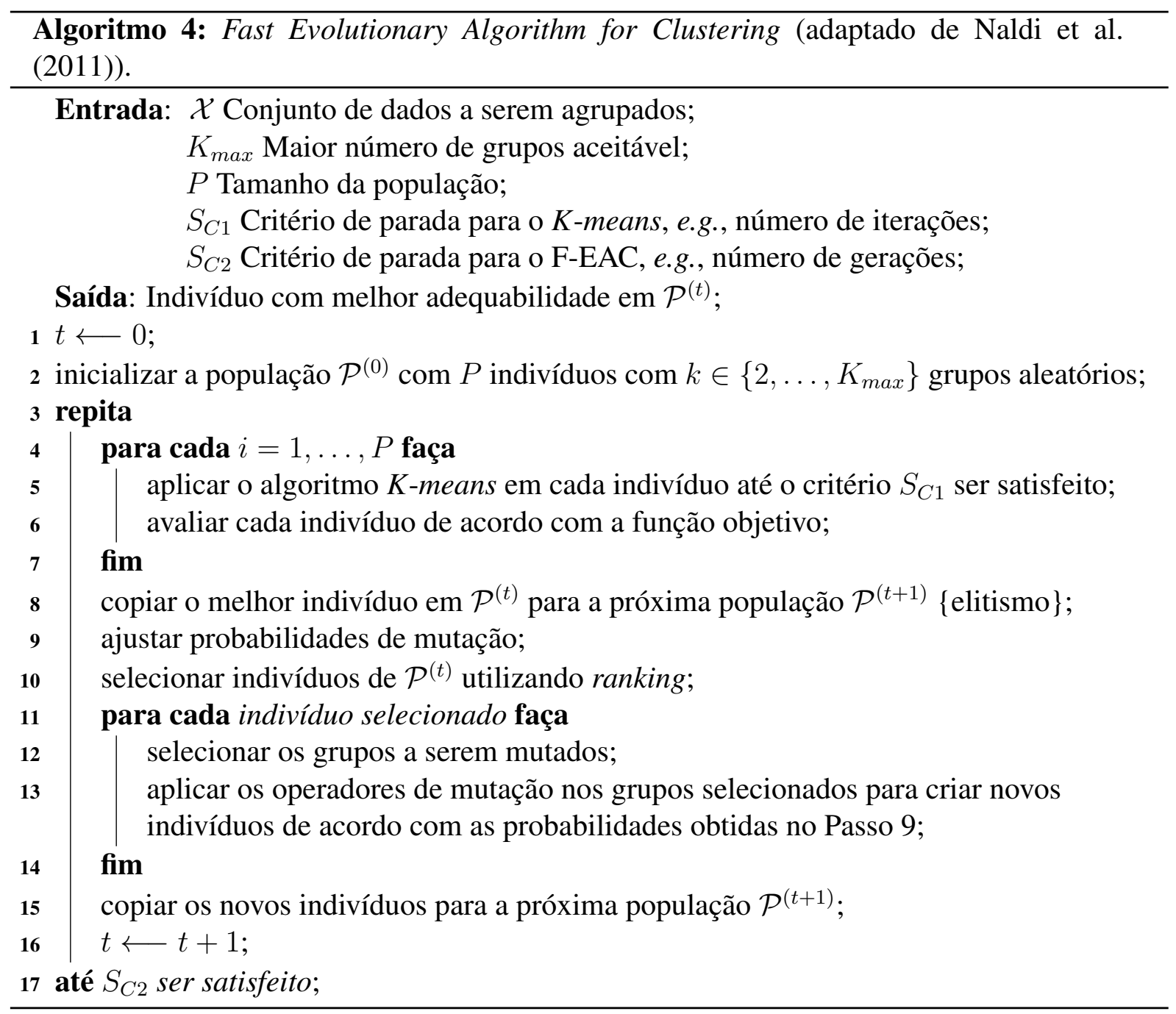

\subsubsection{Algoritmos Evolutivos Baseados em Expectation Maximization para Gaussian Mixture Models}

Apesar do EM para GMM ser um algoritmo amplamente usado na prática, e diversas variantes terem sido desenvolvidas, poucos AEs foram propostos para este problema. Entre eles, alguns buscam tarefas mais especializadas do que obter uma partição probabilística dos dados. Mais especificamente, o algoritmo proposto por Martínez e Virtriá (2000) procura ajustar os componentes da mistura de forma a explicar dados apresentando forma de espiral. No trabalho de Lin e Wang (2005), um AE para uma extensão do GMM, considerando conjuntos fuzzy, é 
analisado. Por fim, Thang et al. (2009) descrevem um AE que busca encontrar outliers nos dados baseando-se nos GMM avaliados. Dado que nesta tese não se tem interesse em modelos com estas particularidades (dados em espiral, conjuntos fuzzy e detecção de outliers), estes trabalhos não são considerados na sequência. Em relação ao desenvolvimento de AEs em conjunto com o EM para GMM, podem-se citar os trabalhos de Pernkopf e Bouchaffra (2005); Tohka et al. (2007); Tawfick et al. (2008) e Nguyen e Cios (2008).

Os algoritmos propostos por Pernkopf e Bouchaffra (2005) e por Nguyen e Cios (2008) Genetic-based Expectation Maximization (GA-EM) e Genetic Algorithm K-means Logarithmic Regression Expectation Maximization (GAKREM), respectivamente — possuem motivação similar aos algoritmos propostos nesta tese. Por tal razão, eles são detalhados a seguir. O algoritmo apresentado por Tohka et al. (2007) supõe que o número de grupos é conhecido, enquanto que o algoritmo proposto por Tawfick et al. (2008) supõe que os grupos são hiperesféricos, i.e., a matriz de covariância das distribuições gaussianas é proporcional à matriz identidade. Por estas razões, ambos os algoritmos são mais simples e menos flexíveis que as propostas a serem abordadas nesta tese.

\subsubsection{Genetic-based Expectation Maximization}

Em Pernkopf e Bouchaffra (2005), um algoritmo denominado Genetic-based Expectation Maximization (GA-EM) foi desenvolvido com o intuito de estimar o número de grupos e otimizar a busca pela solução ótima de um GMM. Cada indivíduo da população representa uma possível solução para o GMM. Os indivíduos são formados por duas partes. Na primeira parte (Parte A), são armazenados $K_{\max }$ bits, sendo $K_{\max }$ o maior número de grupos possível, em que o $k$-ésimo bit indica se o grupo referente a este bit é considerado no GMM que o indivíduo representa. Na segunda parte (Parte B), são armazenados os valores referentes aos parâmetros de cada grupo, ou seja, as médias de cada componente $\left(\boldsymbol{\mu}_{k}\right)$ e as matrizes de covariância $\left(\boldsymbol{\Sigma}_{k}\right)$. Devido aos coeficientes de mistura não serem armazenados, estes são considerados uniformemente distribuídos, i.e., $\pi_{k}=\frac{1}{K}, \forall k \in\{1, \ldots, K\}$, sendo $K$ o número de grupos representado no indivíduo. Portanto, é utilizada a representação baseada em descrição de grupos (Hruschka et al., 2009).

Como função objetivo é utilizada a função logarítmica de verossimilhança - Equação (2.4) - penalizada pelo critério conhecido como Minimum Description Length (MDL) (Rissanen, 1989):

$$
J_{\mathrm{GAEM}}=-L L K(\mathcal{X} \mid \Psi)+\frac{K\left(1+M+\frac{M(M+1)}{2}\right)}{2} \log N .
$$

O melhor indivíduo é aquele que apresenta o menor valor de $J_{\mathrm{GAEM}}{ }^{8}$. Este critério foi escolhido por ser amplamente utilizado como critério de seleção de modelos (Pernkopf e Bouchaffra, 2005). A avaliação dos indivíduos é realizada em duas etapas. Na primeira, $R$ iterações

\footnotetext{
${ }^{8} \mathrm{Na}$ sequência desta tese, o termo MDL será usado para se referenciar a esta função.
} 
do EM são realizadas em cada indivíduo para atualizar seus parâmetros. Na segunda etapa, o valor de MDL de cada indivíduo é obtido. As $R$ iterações do EM são interrompidas se o valor relativo da função logarítmica de verossimilhança - Equação (2.34) - for menor que $\epsilon$, que é um parâmetro definido pelo usuário:

$$
r_{l l k}=\left|\frac{L L K\left(\mathcal{X} \mid \Psi^{(t)}\right)-L L K\left(\mathcal{X} \mid \Psi^{(t+1)}\right)}{L L K\left(\mathcal{X} \mid \Psi^{(t)}\right)}\right|,
$$

na qual $\Psi^{(t)}$ são os parâmetros do GMM na iteração $t$.

Como é conhecido que o algoritmo EM para GMM converge para um ótimo local (McLachlan e Krishnan, 1997) uma estratégia elitista é utilizada, i.e., o melhor indivíduo da geração atual é copiado para a geração subsequente sem sofrer modificações. Para manter esta propriedade, os coeficientes de mistura deste indivíduo são armazenados de uma geração para outra. $\mathrm{O}$ algoritmo é finalizado quando o número de grupos do melhor modelo não muda em cinco gerações consecutivas.

Em cada geração é aplicado um operador de cruzamento que seleciona aleatoriamente dois indivíduos para serem pais, gerando dois filhos. A probabilidade de cruzamento $p_{c}$ determina o número de indivíduos filhos $\lambda$, i.e., $\lambda=p_{c} \cdot P$. O operador consiste no cruzamento em um ponto, o qual seleciona aleatoriamente um ponto $z \in\left\{1, \ldots, K_{\max }\right\}$ na Parte A do indivíduo e troca os valores dos genes à direita desta posição na Parte A e na Parte B entre ambos os indivíduos. É importante notar que este operador pode gerar soluções não desejadas, e.g., com apenas um grupo ou grupos sobrepostos (com a mesma média e possivelmente a mesma matriz de covariância). Desta forma, o operador é orientado a grupos, mas insensível ao contexto. Além disso, o mesmo não é guiado, favorecendo uma busca mais aleatória pela melhor solução.

Para a seleção de quais indivíduos devem permanecer de uma geração para outra, é utilizada a estratégia $(P+\lambda)$, em que $P$ é o número de indivíduos na população e $\lambda$ o número de filhos gerados pelo operador de cruzamento. Este operador escolhe os $P$ melhores indivíduos considerando a população formada pela união dos pais com os filhos.

Dois operadores de mutação são aplicados. No primeiro, denominado mutação forçada, grupos com comportamento similar são forçados a sofrer mutação. Mais especificamente, considerando os vetores de responsabilidades dos componentes $i$ e $j\left(\mathbf{u}_{i}\right.$ e $\left.\mathbf{u}_{j}\right)$, calcula-se o coeficiente de correlação de Pearson entre eles $\left(\rho\left(\mathbf{u}_{i}, \mathbf{u}_{j}\right)\right)$. Caso $\rho\left(\mathbf{u}_{i}, \mathbf{u}_{j}\right)$ seja maior que um limiar ( $t_{\text {correlation }}$ ) um dos grupos é selecionado aleatoriamente para o conjunto de grupos que sofrerão mutação. Após o conjunto estar completo, i.e., $\rho\left(\mathbf{u}_{i}, \mathbf{u}_{j}\right)$ ser calculado para todos $i, j \in\{1, \ldots, K\}$, cada grupo nesse conjunto sofre uma de duas mutações possíveis (esta escolha é realizada aleatoriamente): (i) o grupo é removido redefinindo o seu valor correspondente na Parte A do indivíduo; (ii) a média do grupo é redefinida para as coordenadas de um objeto selecionado aleatoriamente. Pode-se verificar que este operador é guiado e orientado a grupos.

O segundo operador de mutação inverte cada bit da Parte A do indivíduo com probabilidade $p_{m}$, enquanto que na Parte B do indivíduo é inserido um valor aleatório distribuído uniforme- 
mente no intervalo definido pelo menor e maior valor de cada atributo presente nos dados. Além disso, é utilizada uma redução na probabilidade de modificação na Parte B do indivíduo, que é restrita às médias dos grupos, i.e., para as modificações na Parte $\mathrm{B}$ do indivíduo, a probabilidade de mutação é igual a $\left(M+\frac{M(M+1)}{2}\right)^{-1} \cdot p_{m}$.

Assim como no cruzamento, a aplicação de ambos os operadores de mutação pode gerar soluções não desejadas, e.g., com menos de 2 grupos. Caso isso ocorra, a tarefa de remover tais soluções é deixada para o operador de seleção. Nenhuma mutação é realizada no melhor indivíduo (Pernkopf e Bouchaffra, 2005).

Antes de passar para uma nova geração, os indivíduos são analisados para verificar se determinado grupo não é suportado pelos dados. Para tal, é verificado se a soma dos valores do vetor de responsabilidades de um grupo $\left(\mathbf{u}_{i}\right)$ é menor que um limiar $\left(t_{\text {annihilate }}\right)$ definido pelo usuário. De acordo com Pernkopf e Bouchaffra $(2005)^{9}$, este valor deve ser maior que o número de atributos da base de dados. Seguindo o padrão adotado nos experimentos realizados em Pernkopf e Bouchaffra (2005), adota-se $t_{\text {annihilate }}=5 \cdot M$.

O GA-EM é sumarizado no Algoritmo 5. O melhor indivíduo da geração atual é armazenado em $\mathcal{G}_{\min }$ e, após o término do algoritmo, seus parâmetros são otimizados pelo EM e $\left|\mathcal{G}_{\text {min }}\right|$ indica o número de grupos existentes neste indivíduo. Na inicialização, o tamanho da população é definido como $\max \left(K_{\max }-1, P\right)$ sendo que $P$ é o tamanho da população definido pelo usuário. Isto acontece pois, na primeira geração, exige-se que todos os valores possíveis de grupos possuam um indivíduo, ou seja, cada indivíduo representa uma solução com um número diferente de grupos. Cada indivíduo é inicializado aleatoriamente ou utilizando o algoritmo $K$-means. Nas gerações subsequentes, o tamanho da população fica restrito a $P$.

O algoritmo GA-EM possui algumas limitações. Dentre elas, os seus parâmetros, em espe-

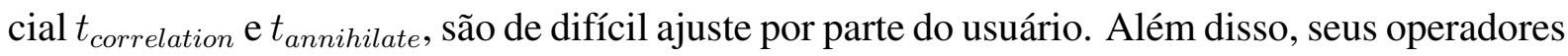
podem gerar soluções inválidas.

\subsubsection{Genetic Algorithm K-means Logarithmic Regression Expectation Maximization}

Em Nguyen e Cios (2008), é proposto um algoritmo denominado Genetic Algorithm $\mathrm{K}$ means Logarithmic Regression Expectation Maximization (GAKREM). Os indivíduos deste algoritmo utilizam codificação inteira com representação baseada em medóide (Hruschka et al., 2009), e.g., o indivíduo [ 147 ] representa uma partição com 3 grupos na qual o medóide do primeiro grupo é o objeto $\mathrm{x}_{1}$, o medóide do segundo grupo é o objeto $\mathrm{x}_{4}$ e o medóide do terceiro grupo é o objeto $\mathbf{x}_{7}$. O número de grupos que podem ser representados em um indivíduo é limitado ao intervalo $[2, \sqrt{N}]$, sendo $N$ o número de objetos da base de dados. Como os parâmetros do GMM que o indivíduo representa não são armazenados, torna-se necessário estimá-los tendo como base os medóides. Para tal, é utilizada uma iteração do algoritmo $K$ means a partir dos medóides.

\footnotetext{
${ }^{9}$ Comunicação pessoal via e-mail.
} 


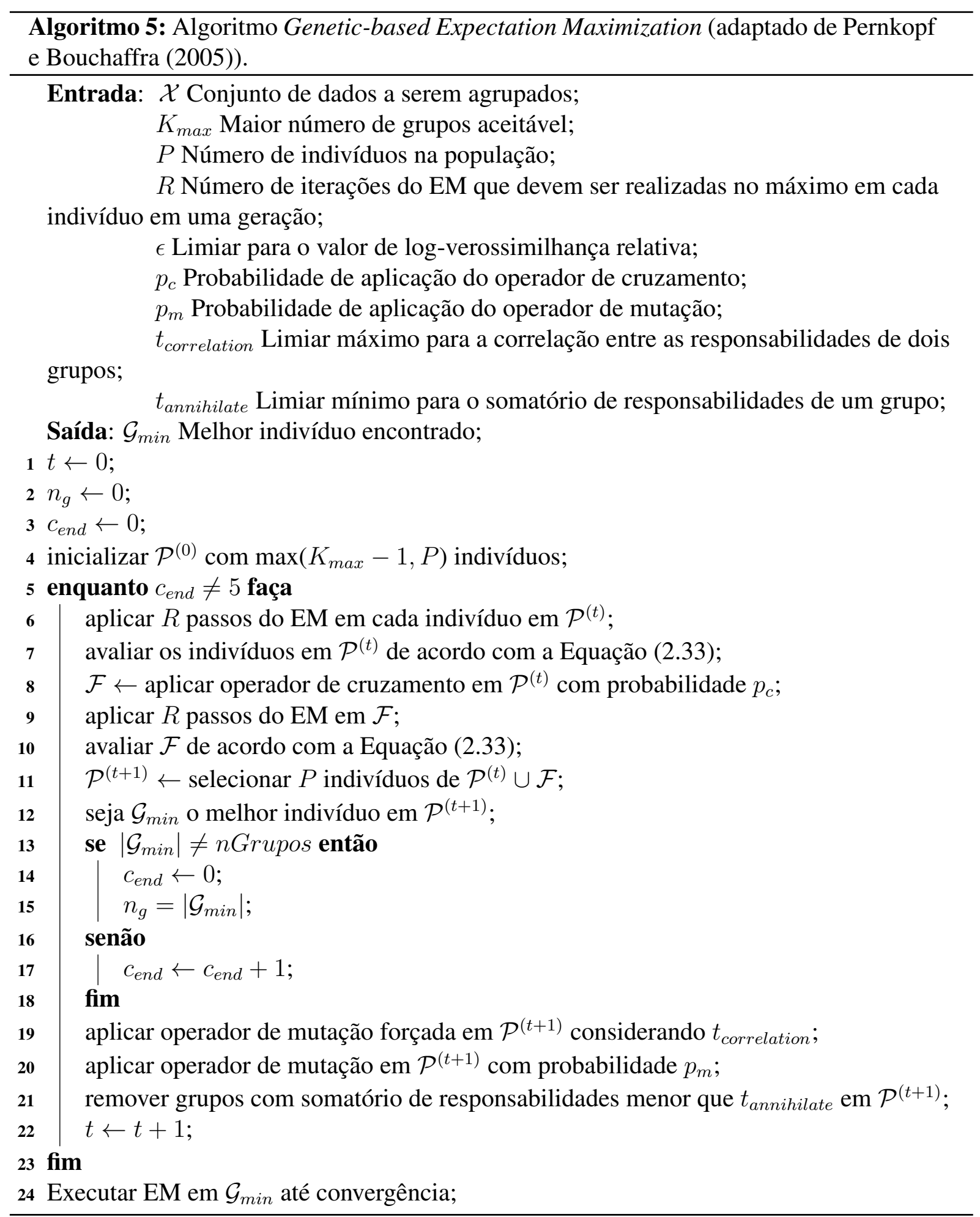


Para a função objetivo, é utilizado o valor esperado, após $r$ iterações do EM, da função logarítmica de verossimilhança do indivíduo, penalizado pelo número de grupos representado. Para encontrar este valor esperado, é utilizada uma função de regressão logarítmica. A adequabilidade do indivíduo $\mathcal{G}$ que representa $K$ grupos, considerando $R$ como o número de iterações executadas pelo EM e $\left\{l_{t}\right\}_{t=1}^{R}$ os valores da função logarítmica de verossimilhança obtidos em cada uma dessas iterações, pode ser definida como (Nguyen e Cios, 2008):

$$
\begin{array}{r}
J_{\text {GAKREM }}=E(\mathcal{G})-\log (K), \\
E(\mathcal{G})=a \log (r)+b, \\
a=\frac{R \sum_{t=1}^{R} t \times l_{t}-\sum_{t=1}^{R} t \times \sum_{t=1}^{R} l_{t}}{R \sum_{t=1}^{R}(t)^{2}-\left(\sum_{t=1}^{R} t\right)^{2}} \\
b=\frac{\sum_{t=1}^{R} l_{t}-a \sum_{t=1}^{R} t}{R} .
\end{array}
$$

A população é formada por $P$ indivíduos inicializados aleatoriamente. Como critério de parada do algoritmo, é adotado um número máximo de 4.000 gerações. Para o cruzamento, dois indivíduos são selecionados utilizando o método da roleta (Eiben e Smith, 2003). A geração do novo indivíduo é definida pelo operador de cruzamento uniforme, em que os medóides são copiados aleatoriamente do primeiro ou segundo indivíduo pai. Note que este operador pode gerar soluções tanto indesejadas quanto inválidas, e.g., um indivíduo com dois grupos centrados no mesmo medóide. Na sequência, o operador de mutação é aplicado no indivíduo gerado com uma dada taxa de probabilidade $\left(p_{m}\right)$. Este operador aleatoriamente cria um grupo considerando como medóide um objeto escolhido aleatoriamente ou remove um grupo da partição representada pelo indivíduo.

O GAKREM é descrito no Algoritmo 6. As principais limitações deste algoritmo são: demora a convergir, dado que a cada geração apenas um novo indivíduo é gerado e não há refinamento das partições dos indivíduos atuais; é difícil definir o valor do parâmetro $r$ que influencia a adequabilidade do indivíduo; o critério de penalização pelo número de componentes não tem motivação teórica clara.

\subsection{Considerações Finais}

Neste capítulo, uma breve introdução sobre GMMs foi realizada. Além disso, foram descritas duas abordagens para se evitar problemas conhecidos na estimação dos parâmetros de GMMs: operadores de união e divisão de componentes e Algoritmos Evolutivos (AEs). O uso 


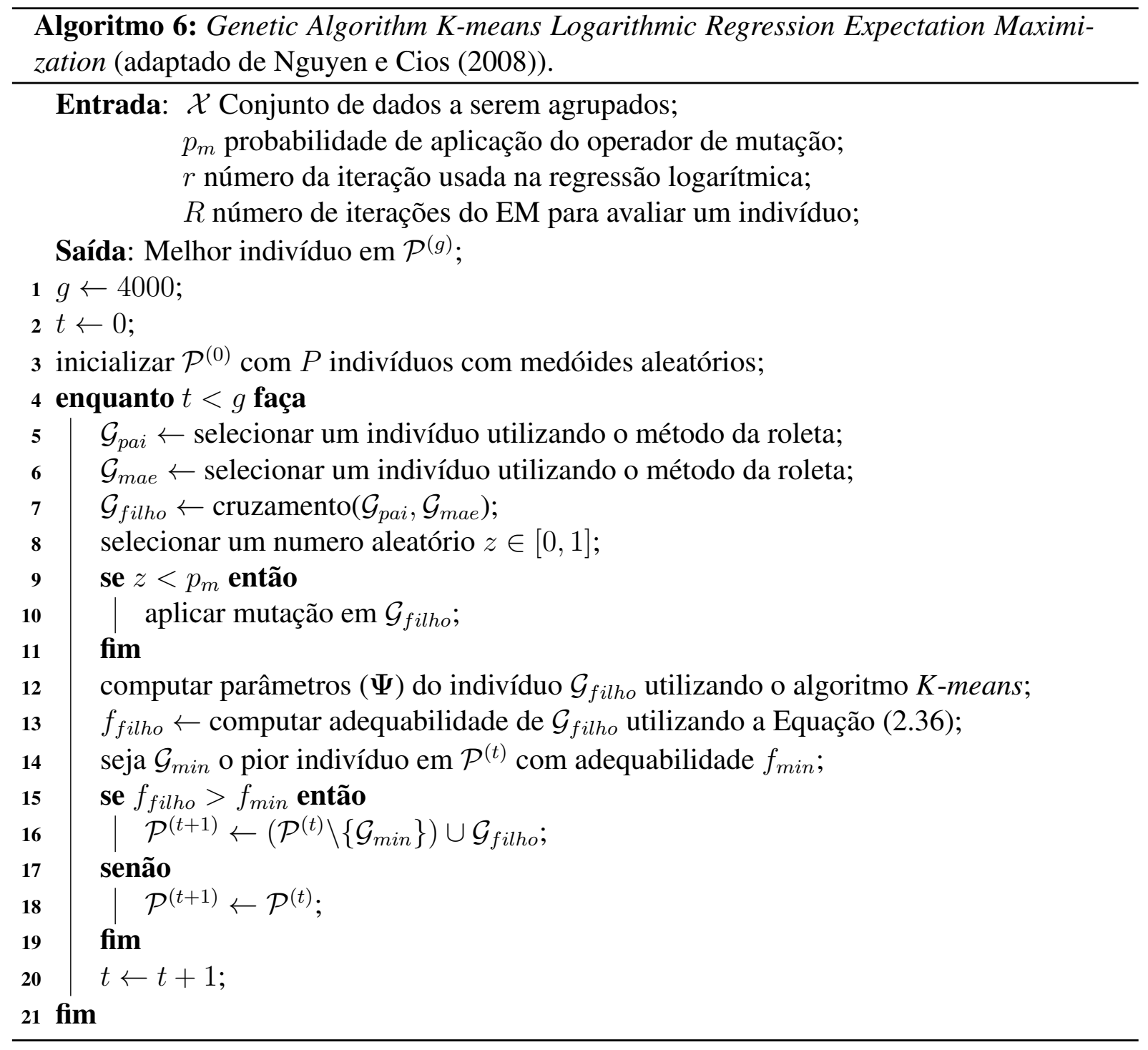


conjunto destas técnicas deu origem ao algoritmo Evolutionary Split \& Merge Algorithm for Expectation Maximization (ESM-EM) (Covões e Hruschka, 2011), descrito na Seção 3.2, e serviu de fundamentação para o algoritmo Feasible-Infeasible Evolutionary Create \& Eliminate Algorithm for Expectation Maximization (FIECE-EM), apresentado no Capítulo 7. 


\section{Algoritmos Evolutivos para Modelos de Mistura de Gaussianas Não Supervisionados}

\subsection{Considerações Iniciais}

Conforme discutido no Capítulo 2, dois problemas são comumente encontrados no uso prático de Gaussian Mixture Models (GMMs): dificuldade em se evitar ótimos locais na estimação dos parâmetros e a necessidade da definição do número de componentes a priori. Neste capítulo é descrita uma das contribuições desenvolvidas nesta tese que busca remediar tais problemas. Especificamente, foi desenvolvido um algoritmo evolutivo para GMMs que utiliza operadores de união e divisão de componentes (detalhes sobre estes tipos de operadores estão descritos na Seção 2.3), denominado Evolutionary Split \& Merge Algorithm for Expectation Maximization (ESM-EM) (Covões e Hruschka, 2011). Tal algoritmo foi desenvolvido levando em consideração experiências e resultados do algoritmo F-EAC (Campello et al., 2009; Horta e Campello, 2009; Naldi et al., 2011).

Embora o algoritmo EM seja útil em diversos problemas e não apenas no contexto de agrupamento, neste capítulo o mesmo será referenciado como um algoritmo de agrupamento devido ao contexto específico relacionado ao trabalho. Além disso, considera-se que o modelo utilizado para o particionamento dos dados é um Gaussian Mixture Model, i.e., cada grupo dos dados é oriundo de uma distribuição de probabilidades gaussiana e, portanto, os termos grupo e componente são utilizados com o mesmo significado. 


\subsection{Evolutionary Split \& Merge Algorithm for Expectation Ma- ximization}

O ESM-EM combina propriedades dos algoritmos GA-EM (Pernkopf e Bouchaffra, 2005) e F-EAC (Campello et al., 2009; Naldi et al., 2011) com procedimentos de divisão e união de componentes. Especificamente, e diferentemente de outros algoritmos da literatura (i.e., Pernkopf e Bouchaffra (2005) e Nguyen e Cios (2008)), são adotados operadores específicos ao contexto guiados por funções de qualidade relacionadas à adequabilidade do indivíduo. Portanto, o ESM-EM tende a favorecer uma busca guiada (probabilisticamente) em comparação com abordagens existentes, que realizam a busca de uma forma mais aleatória.

Para codificar os indivíduos, é necessário uma representação capaz de (i) lidar com um número variável de grupos e (ii) definir precisamente o GMM representado pelo indivíduo. Feitas tais observações, e tomando $K_{\max }$ como o maior número de grupos aceitável, foi adotada uma codificação baseada em grupo ${ }^{1}$. Nesta codificação, os seguintes dados são codificados em cada genótipo no ESM-EM: parâmetros de cada distribuição gaussiana $\left(\left\{\boldsymbol{\mu}_{k}, \boldsymbol{\Sigma}_{k}\right\}_{k=1}^{K_{\max }}\right)$ e os coeficientes de mistura de cada distribuição $\left(\left\{\pi_{k}\right\}_{k=1}^{K_{\max }}\right)$. A limitação de determinar o número máximo de grupos pode ser removida utilizando uma codificação de tamanho de variável, caso desejado.

Por considerar apenas cenários em que o número de grupos não é conhecido, a função logarítmica de verossimilhança (Equação 2.4) - não pode ser utilizada diretamente como função objetivo, pois ela é não-decrescente em relação ao número de grupos (Figueiredo e Jain, 2002). Por este motivo, neste trabalho, esta é acrescida do critério de penalização conhecido por Minimum Description Length (MDL) (Rissanen, 1989). Diversos destes critérios são discutidos na literatura - para o leitor interessado sugere-se o trabalho de McLachlan e Peel (2000) - e o mesmo pode ser substituído caso algum outro critério seja preferido para cada aplicação prática específica. Desta forma, a função objetivo do ESM-EM é a mesma do algoritmo GA-EM Equação (2.33). A escolha por esta função é justificada por ser bem estabelecida na literatura (McLachlan e Peel, 2000; Figueiredo e Jain, 2002; Pernkopf e Bouchaffra, 2005) e por ser possível realizar a decomposição necessária para avaliar a contribuição individual de cada grupo para a qualidade da solução final — conforme pode-se notar da Equação 3.1, sendo $K$ o número de grupos do indivíduo e $k$ o grupo sendo avaliado. Além disso, a adoção desse critério facilita as comparações com o algoritmo GA-EM (Pernkopf e Bouchaffra, 2005).

$$
D_{\mathrm{LLK}}(k)=\sum_{n=1}^{n} \frac{\pi_{k} \mathcal{N}\left(\mathbf{x}_{n} \mid \boldsymbol{\mu}_{k}, \boldsymbol{\Sigma}_{k}\right)}{\sum_{k_{1}=1}^{K} \pi_{k_{1}} \mathcal{N}\left(\mathbf{x}_{n} \mid \boldsymbol{\mu}_{k_{1}}, \boldsymbol{\Sigma}_{k_{1}}\right)}\left\{\ln \pi_{k}+\ln \mathcal{N}\left(\mathbf{x}_{n} \mid \boldsymbol{\mu}_{k}, \boldsymbol{\Sigma}_{k}\right)\right\} .
$$

As principais etapas do algoritmo ESM-EM, sumarizado no Algoritmo 7, são: (i) inicialização (Passo 1); (ii) refinamento (Passo 4); (iii) seleção de indivíduos para mutação e sobrevivên-

\footnotetext{
${ }^{1}$ Cluster-based encoding (Hruschka et al., 2009).
} 
cia (Passos 5 e 11); (iv) tipo de mutação e sua seleção (Passos 7 e 8). Nas próximas subseções, estes passos são discutidos em mais detalhes, bem como os possíveis componentes que podem ser usados, dando origem a diferentes variantes para o ESM-EM.

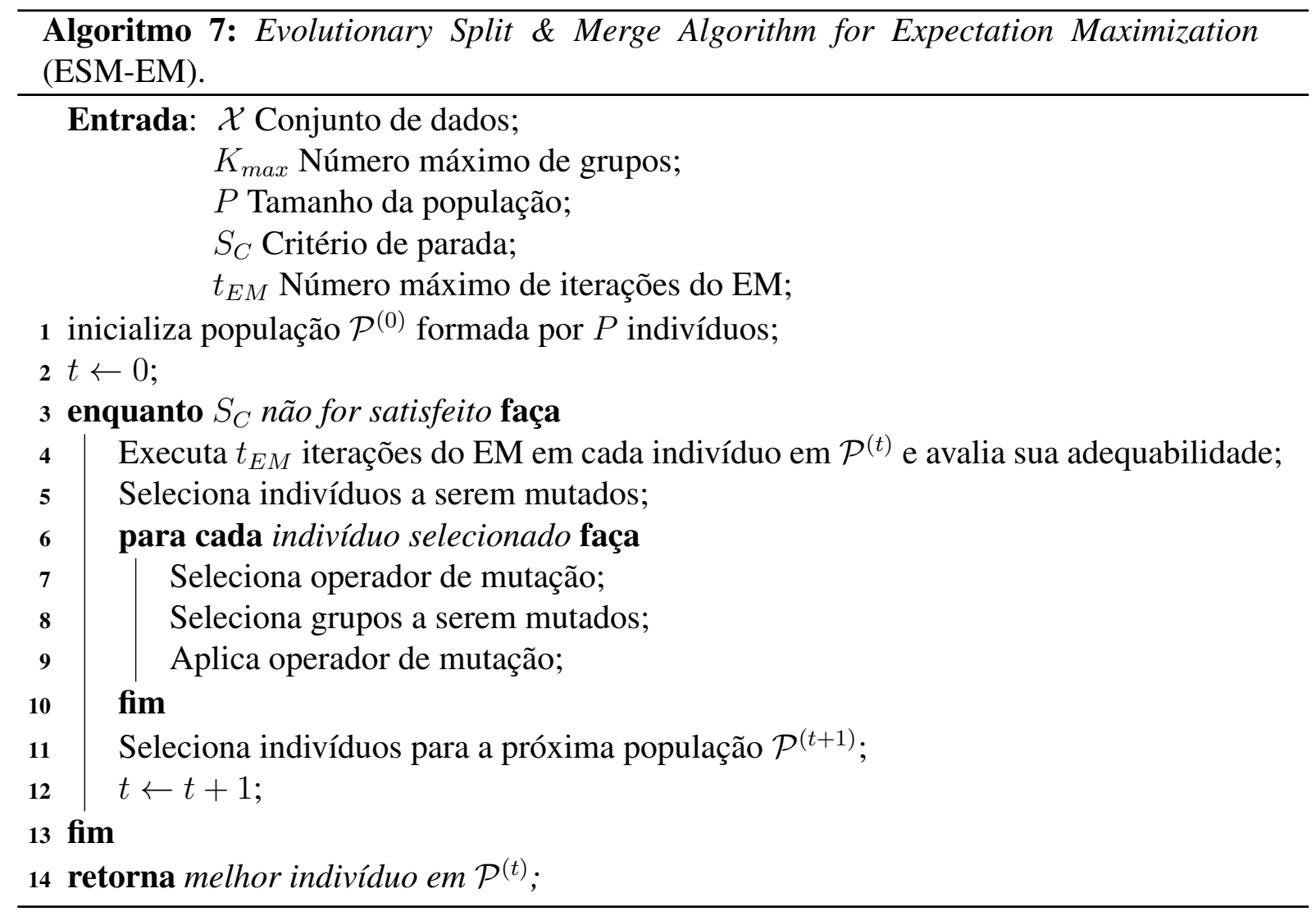

\subsubsection{Inicialização}

Cada indivíduo é inicializado para representar uma partição obtida pelo algoritmo $K$-meansusando objetos selecionados aleatoriamente como sementes. O número de grupos é definido de forma determinística. Especificamente, $P$ indivíduos são inicializados com o número de grupos espaçados linearmente entre 2 e $K_{\max }$, assegurando que as soluções iniciais estão distribuídas uniformemente entre os possíveis valores de número de grupos. Diferentemente do trabalho realizado por Pernkopf e Bouchaffra (2005) no qual, se necessário, aumenta-se a população inicial para garantir que tenha um indivíduo com cada número de grupos possível, o ESM-EM utiliza uma solução com melhor compromisso entre diversidade de partições e custo computacional.

\subsubsection{Refinamento}

No Passo 4, ESM-EM executa um número $\left(t_{E M}\right)$ de iterações do EM em cada indivíduo da população e os indivíduos são avaliados pela função objetivo (Equação (2.33)). O algoritmo ESM-EM não utiliza operadores de recombinação (crossover). No entanto, o refinamento de 
soluções pode ser visto como um procedimento de busca local, favorecendo o que comumente se denomina de exploitation (Črepinšek et al., 2013).

\subsubsection{Seleção de Indivíduos para Mutação e Sobrevivência}

Como operador de seleção é utilizada a seleção $(\mu+\lambda)$ (Bäck et al., 2000a). A cada geração, $P$ indivíduos são gerados aplicando-se mutação em cada indivíduo em $\mathcal{P}^{(t)}$, obtendo-se $\mathcal{P}_{\text {mut }}$. Os melhores $P$ indivíduos da população formada por $\mathcal{P}^{(t)} \cup \mathcal{P}_{\text {mut }}$ sobrevivem e formam $\mathcal{P}^{(t+1)}$ — uma abordagem similar foi adotada em Pernkopf e Bouchaffra (2005). Existem duas razões principais para adotar este procedimento: (i) como o algoritmo EM é conhecido por convergir lentamente (Redner e Walker, 1984), uma seleção mais conservadora favorece o refinamento acumulado necessário para um melhor ajuste dos parâmetros; (ii) dado que os operadores de mutação baseados em divisão e união de componentes são relativamente agressivos, no sentido de que podem realizar grandes saltos no espaço de soluções, a maior desvantagem de uma seleção conservadora, i.e., a convergência prematura, tem seu efeito reduzido.

\subsubsection{Operadores de Mutação}

Seguindo a taxonomia apresentada em Hruschka et al. (2009) e resumida na Seção 2.4, os operadores de mutação do algoritmo ESM-EM são guiados e orientados a grupos. Tais características são desejáveis por tornarem as mutações mais eficazes na exploração do espaço de busca.

Os operadores de mutação do ESM-EM consistem na aplicação de procedimentos de união e divisão de grupos/componentes. Para escolher qual operador será aplicado é utilizada a abordagem adotada no F-EAC apresentada na Seção 2.4.1.1. Nesta abordagem, caso o indivíduo tenha melhorado de uma geração $t$ para uma geração $t+1$, o mesmo operador é aplicado; caso contrário o outro operador é utilizado. Em outros casos (e.g., primeira geração) o operador é determinado aleatoriamente.

Para a união de componentes, é considerado o procedimento descrito por Zhang et al. $(2003)^{2}$ que é sumarizado no Algoritmo 8. Para a divisão de componentes, dois operadores são considerados: divisão baseada em Singular Value Decomposition (SVD) (Zhang et al., 2003) ${ }^{3}$, descrito no Algoritmo 9, e o operador Variance based Splitting (VS), descrito na sequência.

Este novo operador, VS, é baseado na divisão do grupo levando em consideração a variância do grupo em cada atributo e é descrito no Algoritmo 10, sendo que $\sigma_{i j}^{(s)}$ representa o valor da $i$ ésima linha e $j$-ésima coluna da matriz $\Sigma_{s}$, i.e., a covariância entre os atributos $i$ e $j$, e trace $\left(\Sigma_{s}\right)$ é a função que calcula a soma dos valores na diagonal principal da matriz $\Sigma_{s}$. O algoritmo de agrupamento $K$-means é utilizado para estimar as matrizes de covariância dos novos grupos, visando tornar desnecessária a definição dos parâmetros $\alpha, u, \beta$ e, ao mesmo tempo, visando

\footnotetext{
${ }^{2}$ Seção 2.3.1.

${ }^{3}$ Seção 2.3.2.
} 


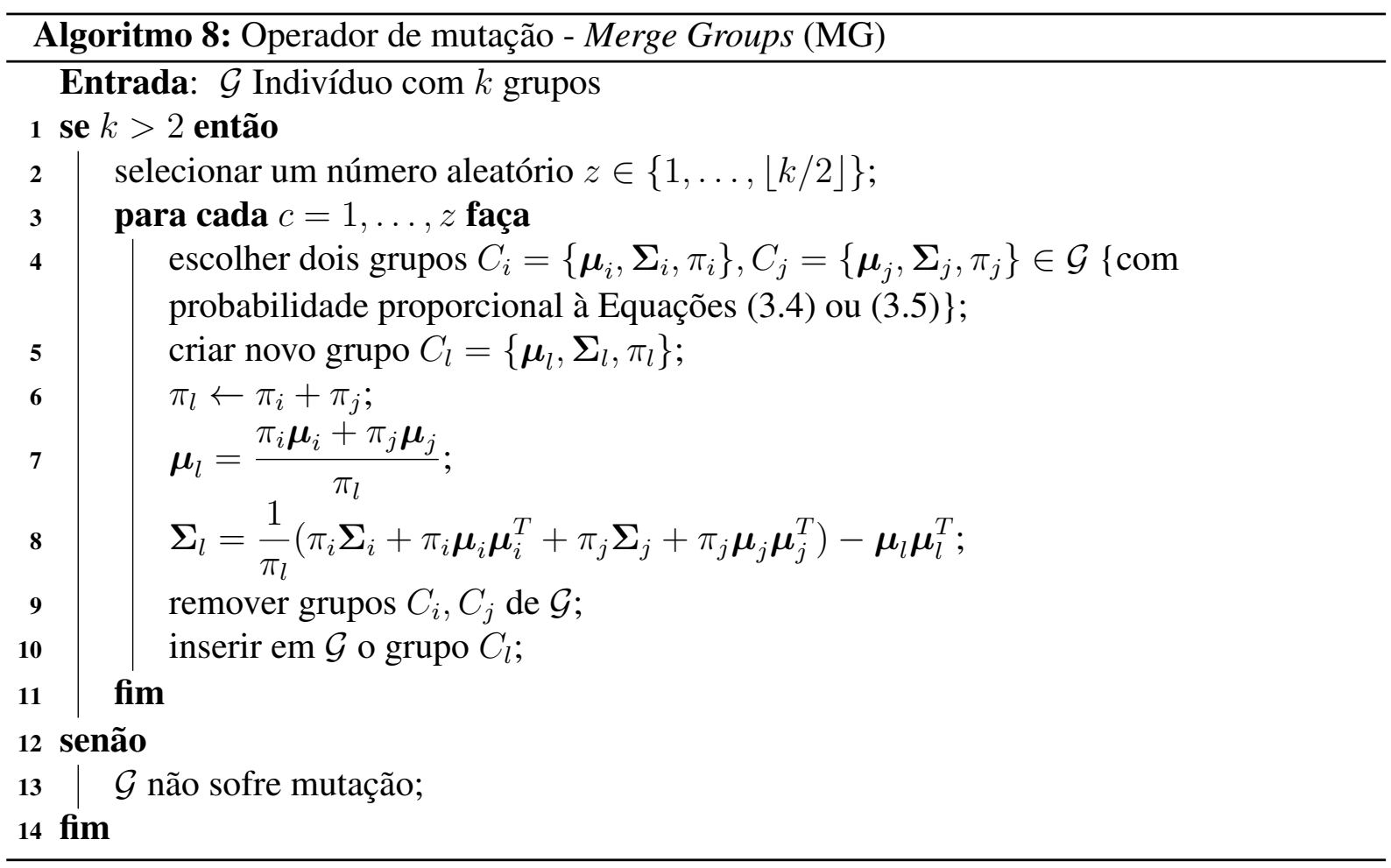

reduzir o custo computacional envolvido no operador SVDS. Além disso, com a aplicação do $K$-means as posições das novas médias $\boldsymbol{\mu}_{i}$ e $\boldsymbol{\mu}_{j}$ são atualizadas para o centro de massa dos pontos mais próximos. Levando em consideração o custo necessário para a aplicação do $K$ means, o custo computacional desse operador é de $O\left(t_{k m} \cdot\left|C_{s}\right| \cdot M\right)$, sendo que $t_{k m}$ é o número de iterações do $K$-means e $\left|C_{s}\right|$ denota os objetos que têm maior probabilidade de se originarem do componente $s$, que está sendo dividido. É importante ressaltar que o $K$-means pode gerar grupos vazios durante a sua execução. Devido a esse efeito ser indesejado, caso isto ocorra, o centróide do grupo vazio é movido para o objeto mais distante em relação ao centro do seu grupo.

Embora seja possível adaptar os operadores SVDS e VS para que dividam um grupo em mais que dois grupos, a literatura sugere que a divisão de um grupo em dois proporciona bons resultados. Pode-se citar, por exemplo, o algoritmo Bisecting $K$-means (Tan et al., 2005), que é uma extensão do $K$-means e que apresentou bons resultados em experimentos relacionados à mineração de textos (Steinbach et al., 2000). É importante ressaltar que, considerando a perspectiva de algoritmos evolutivos, na qual boas soluções são mantidas da geração atual para a geração seguinte, uma nova aplicação dos operadores na mesma solução em uma geração futura permite gerar divisões em mais grupos.

Para definir a probabilidade de determinado componente (grupo) ser dividido, dois critérios foram considerados: Partial Log-Likelihood (PLL) - Equação (3.1) - e o Local Kullback Divergence (LKD) - Equação (2.15). O uso da medida PLL repete a metodologia utilizada com sucesso no algoritmo F-EAC, em que a função objetivo é decomposta em partes e essa decomposição é utilizada para guiar os grupos a serem modificados. No entanto, é conhecido 


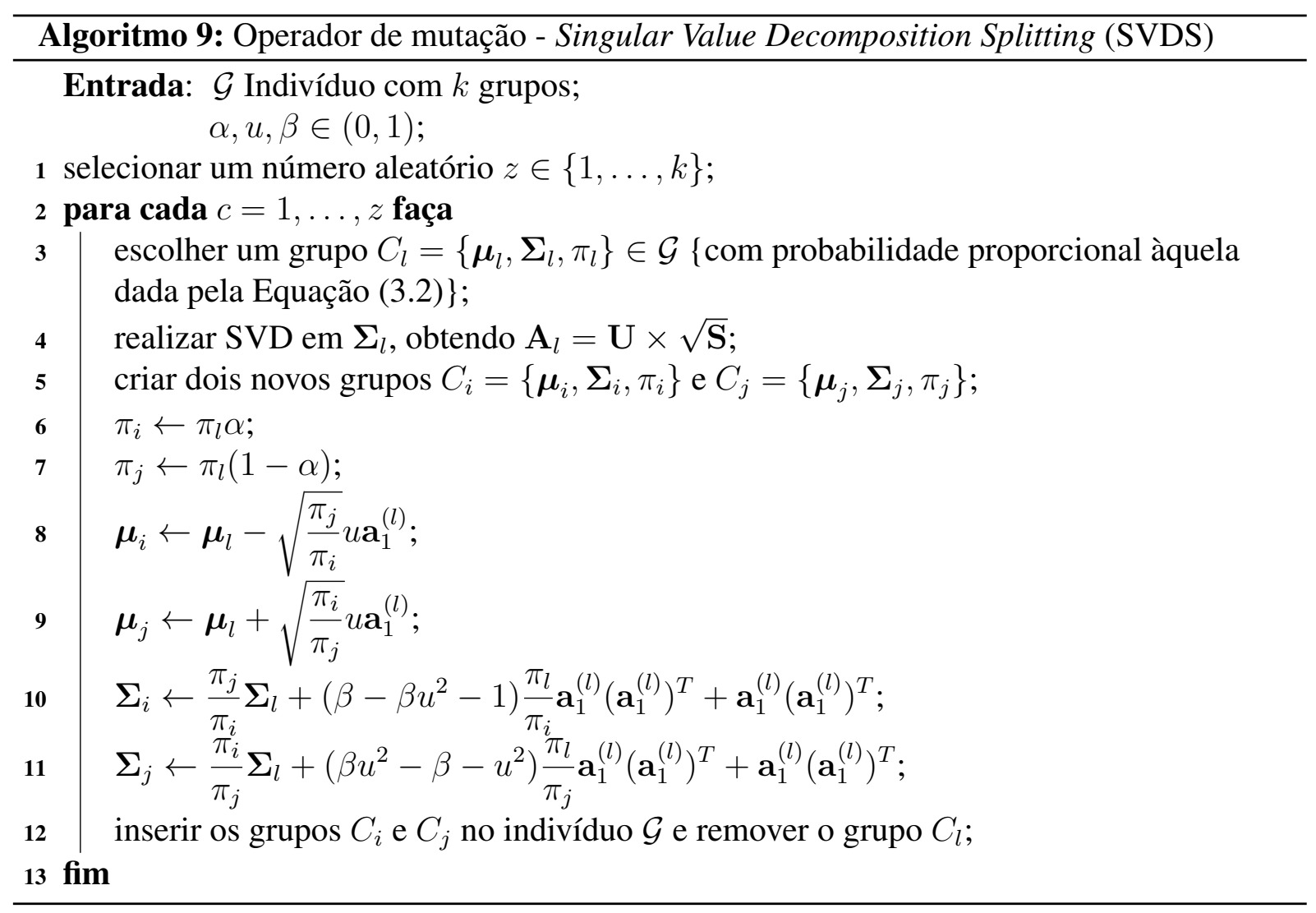

que o máximo global da verossimilhança corresponde a soluções singulares com variância igual a zero (Ueda et al., 2000; Bishop, 2006). Portanto, essa função tem um viés favorável a grupos com pouca variância, ou seja, coesos. Embora grupos com essa característica sejam interessantes do ponto de vista de agrupamento de dados, esse viés pode direcionar a busca para a solução trivial de um grupo por objeto (grupos singletons), o que não é desejado. Com isto, justifica-se a investigação da aplicação do critério LKD que é amplamente utilizado na literatura de SM (Ueda et al., 2000; Zhang et al., 2003; Wang et al., 2004; Zhang et al., 2004a; Blekas e Lagaris, 2007). Seja $D_{\text {split }}$ um dos dois critérios mencionados, a probabilidade do grupo $C_{i}$ sofrer uma mutação de divisão é dada por:

$$
P_{m}\left(C_{i}\right)=\frac{\operatorname{rank}\left(D_{\text {split }}(i \mid \boldsymbol{\Psi})\right)}{\sum_{k=1}^{K} \operatorname{rank}\left(D_{\text {split }}(k \mid \Psi)\right)}
$$

Conforme mencionado anteriormente, para a união de grupos é necessário definir critérios que quantifiquem a qualidade da união de pares de grupos. Foram analisados dois critérios: sobreposição entre grupos e correlação entre responsabilidades.

Na Seção 2.3.1 foi apresentada uma medida de sobreposição desenvolvida por Li e Ma (2008). No entanto, esta medida depende de um parâmetro $\epsilon$, difícil de ser definido pelo usuário, e do cálculo da distância de Mahalanobis entre dois grupos, a qual é custosa computacionalmente. Para suprir estas limitações, uma nova medida de sobreposição foi desenvolvida. 


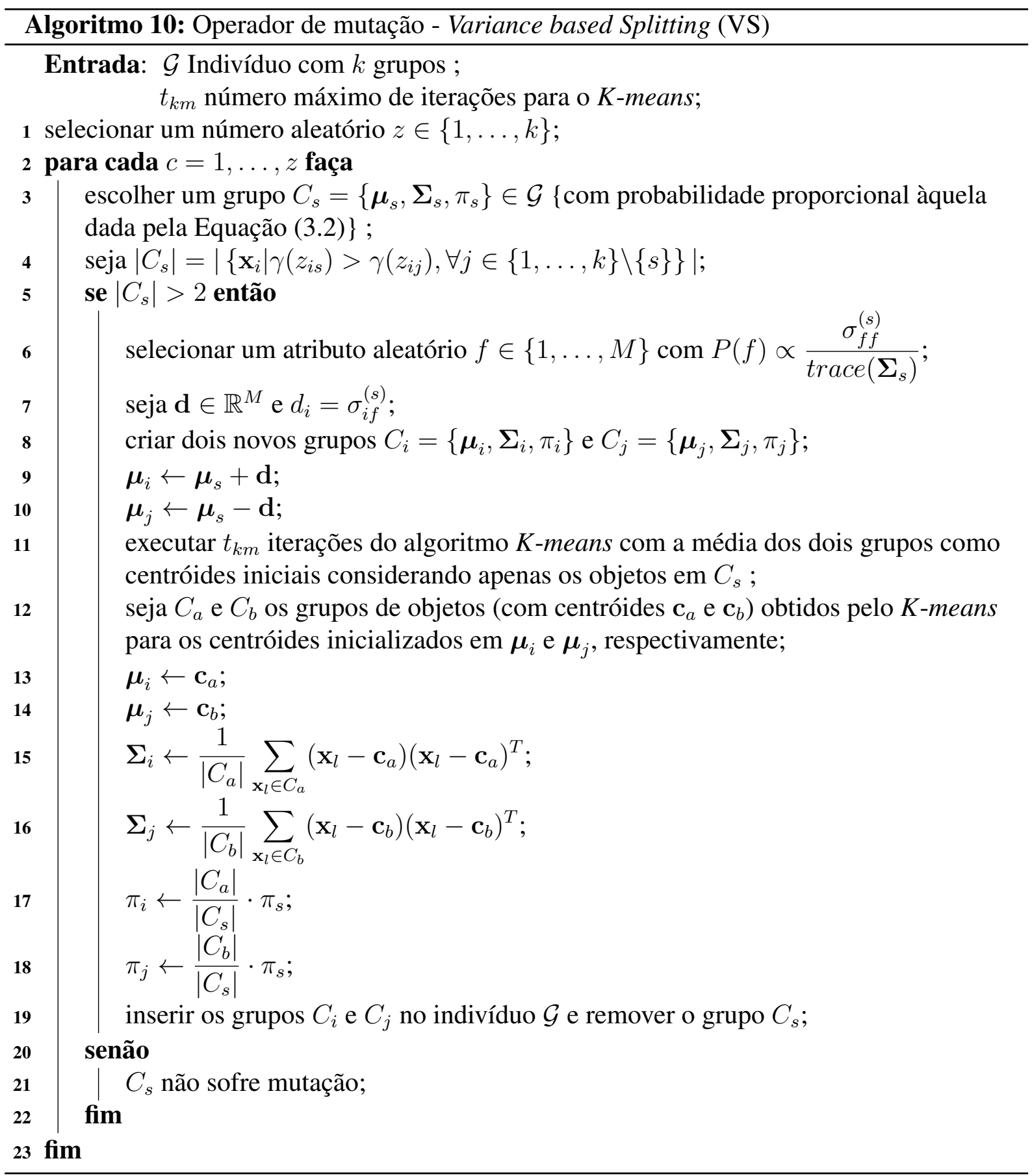


Seja $\mathcal{A}$ o conjunto de todos os objetos que seriam rotulados ${ }^{4}$ para o grupo $a$ e que tenham o grupo $b$ como vizinho mais próximo, i.e., $\mathcal{A}=\left\{\mathbf{x}_{i} \mid \gamma\left(z_{i a}\right) \geq \gamma\left(z_{i b}\right) \geq \gamma\left(z_{i c}\right), \forall c \notin\{a, b\}\right\}$ e $\mathcal{B}$ o conjunto de todos os objetos que seriam rotulados para o grupo $b$ e que tenham o grupo $a$ como vizinho mais próximo, i.e., $\mathcal{B}=\left\{\mathbf{x}_{i} \mid \gamma\left(z_{i b}\right) \geq \gamma\left(z_{i a}\right) \geq \gamma\left(z_{i c}\right), \forall c \notin\{a, b\}\right\}$. A medida $U_{\text {overlap } 2}(a, b)$ é definida como:

$$
U_{\text {overlap } 2}(a, b)=\frac{1}{2} \cdot\left(\frac{\sum_{\mathbf{x}_{i} \in \mathcal{A}}\left[1-\left(\gamma\left(z_{i a}\right)-\gamma\left(z_{i b}\right)\right)\right]}{|\mathcal{A}|}+\frac{\sum_{\mathbf{x}_{i} \in \mathcal{B}}\left[1-\left(\gamma\left(z_{i b}\right)-\gamma\left(z_{i a}\right)\right)\right]}{|\mathcal{B}|}\right)
$$

O par de grupos que maximiza $U_{\text {overlap } 2}$ é o par de grupos que têm maior sobreposição entre si. Portanto, considerando um ranking no qual o par de grupos com menor sobreposição está na posição 1, a probabilidade de um par de grupos sofrer mutação de acordo com esse critério é dada por:

$$
P_{m}\left(C_{i}, C_{j}\right)=\frac{\operatorname{rank}\left(U_{\text {overlap } 2}(i, j)\right)}{\sum_{m=1}^{K-1} \sum_{l=m+1}^{K} \operatorname{rank}\left(U_{\text {overlap } 2}(m, l)\right)}
$$

Para o uso deste critério, torna-se necessário o cálculo da medida entre os $\left(\begin{array}{c}K \\ 2\end{array}\right)$ pares de grupos, tendo para cada par o custo computacional estimado em $O(N \cdot K)$ - supondo que as responsabilidades de cada componente sob cada objeto já foram previamente calculadas.

O segundo critério, denominado de coeficiente de correlação entre responsabilidades, já foi utilizado em outros trabalhos (Zhang et al., 2003; Pernkopf e Bouchaffra, 2005) e foi descrito na Seção 2.3.1 - Equação (2.8). O par de grupos com maior valor absoluto de correlação é o melhor candidato para a união. O custo computacional é de $O(N)$ para cada par de grupos. De forma análoga ao critério de sobreposição, a probabilidade de um par de grupos sofrer mutação de acordo com a correlação pode ser definida como:

$$
P_{m}\left(C_{i}, C_{j}\right)=\frac{\operatorname{rank}\left(\left|U_{\text {correlation }}(i, j)\right|\right)}{\sum_{m=1}^{K-1} \sum_{l=m+1}^{K} \operatorname{rank}\left(\left|U_{\text {correlation }}(m, l)\right|\right)}
$$

Para exemplificar o uso destes operadores, na próxima subseção são apresentados alguns exemplos de aplicação em situações típicas.

\subsubsection{Exemplos de Aplicação}

Considere um cenário no qual há 1.000 objetos gerados pelo seguinte modelo:

$$
\boldsymbol{\mu}_{1}=\left[\begin{array}{ll}
1 & 1
\end{array}\right], \quad \boldsymbol{\mu}_{2}=\left[\begin{array}{ll}
3 & 3
\end{array}\right]
$$

${ }^{4}$ Considerando a regra de rotular cada objeto como oriundo do componente com maior responsabilidade. 


$$
\begin{aligned}
& \boldsymbol{\Sigma}_{1}=\left[\begin{array}{cc}
0,2 & 0 \\
0 & 0,2
\end{array}\right], \\
& \boldsymbol{\Sigma}_{2}=\left[\begin{array}{cc}
0,05 & 0 \\
0 & 0,05
\end{array}\right] \text {, } \\
& \pi_{1}=\frac{1}{2} \text {, } \\
& \pi_{2}=\frac{1}{2} \text {, }
\end{aligned}
$$

e que a partição atual, ilustrada na Figura 3.1(a), seja formada por três grupos com os seguintes parâmetros:

$$
\begin{aligned}
& \boldsymbol{\mu}_{1}=\left[\begin{array}{ll}
0,5 & 0,5
\end{array}\right], \quad \boldsymbol{\mu}_{2}=\left[\begin{array}{ll}
1,5 & 1,5
\end{array}\right], \quad \boldsymbol{\mu}_{3}=\left[\begin{array}{ll}
3 & 3
\end{array}\right], \\
& \boldsymbol{\Sigma}_{1}=\left[\begin{array}{cc}
0,1 & 0 \\
0 & 0,1
\end{array}\right], \quad \boldsymbol{\Sigma}_{2}=\left[\begin{array}{cc}
0,1 & 0 \\
0 & 0,1
\end{array}\right], \quad \boldsymbol{\Sigma}_{3}=\left[\begin{array}{cc}
0,1 & 0 \\
0 & 0,1
\end{array}\right] \text {, } \\
& \pi_{1}=\frac{1}{4}, \quad \pi_{2}=\frac{1}{4}, \quad \pi_{3}=\frac{1}{2} .
\end{aligned}
$$

É fácil verificar que os grupos $C_{1}$ e $C_{2}$ são os mais indicados para a união. Aplicando a Equação (3.3) aos pares de grupos, obtêm-se: $U_{\text {overlap } 2}(1,2) \approx 0,17, U_{\text {overlap } 2}(1,3)$ e $U_{\text {overlap } 2}(2,3) \approx$ 0, 04. Portanto, conforme esperado, o par formado por $C_{1}$ e $C_{2}$ teria maior probabilidade de ser mutado de acordo com a Equação (3.4). Aplicando a Equação (2.8) em todos os pares verificase que $U_{\text {correlation }}(1,2) \approx 0,14, U_{\text {correlation }}(1,3) \approx 0$ e $U_{\text {correlation }}(2,3) \approx 0$. Portanto, considerando ambos os critérios, o par de grupos com maior probabilidade de ser unido é formado pelos grupos $C_{1}$ e $C_{2}$, como esperado. Assumindo que foi selecionado o par de grupos $C_{1}$ e $C_{2}$ para a união, a aplicação do operador de união resultaria na geração de um novo grupo $\left(C_{k}\right)$ com os seguintes parâmetros:

(i) $\pi_{k}=0,25+0,25=0,5$;

(ii) $\boldsymbol{\mu}_{k}=\frac{0,25 \times\left[\begin{array}{ll}0,5 & 0,5\end{array}\right]+0,25 \times\left[\begin{array}{ll}1,5 & 1,5\end{array}\right]}{0,5}=\left[\begin{array}{ll}1 & 1\end{array}\right]$;

(iii) $\boldsymbol{\Sigma}_{k}=\frac{1}{0,5}\left(0,25 \times \operatorname{diag}([0,1 \quad 0,1])+0,25 \times\left[\begin{array}{ll}0,5 & 0,5\end{array}\right] \times\left[\begin{array}{ll}0,5 & 0.5\end{array}\right]^{T}+0,25 \times\right.$ $\left.\operatorname{diag}\left(\left[\begin{array}{ll}0,1 & 0,1\end{array}\right]\right)+0,25 \times\left[\begin{array}{ll}1,5 & 1,5\end{array}\right] \times\left[\begin{array}{ll}1,5 & 1,5\end{array}\right]^{T}\right)-\left[\begin{array}{ll}1 & 1\end{array}\right] \times\left[\begin{array}{ll}1 & 1\end{array}\right]^{T}=\left[\begin{array}{ll}0,6 & 0,5 \\ 0,5 & 0,6\end{array}\right]$.

É possível verificar que o novo grupo $C_{k}$ é similar ao grupo correto e, com a aplicação do operador, a partição encontra-se agora mais próxima do modelo que originou os dados. A Figura 3.1(b) ilustra a partição resultante após a aplicação do operador.

Para exemplificar a aplicação a divisão de grupos, considere o seguinte cenário no qual há 1.500 objetos gerados pelo seguinte modelo: 


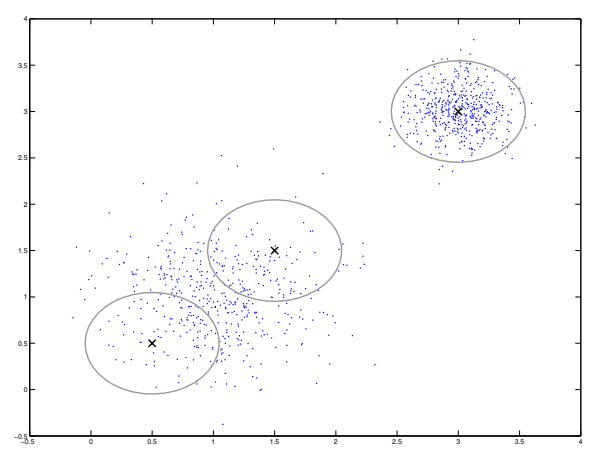

(a) Antes da aplicação do operador.

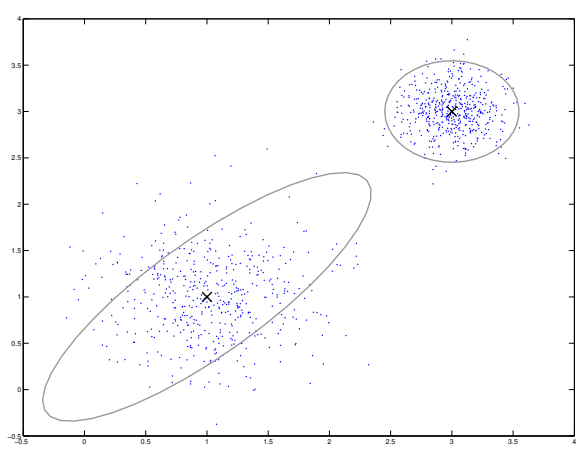

(b) Após a aplicação do operador.

Figura 3.1: Exemplo da aplicação do operador Merge Groups.

$$
\begin{aligned}
& \boldsymbol{\mu}_{1}=\left[\begin{array}{ll}
1 & 1
\end{array}\right] \text {, } \\
& \boldsymbol{\mu}_{2}=\left[\begin{array}{ll}
1 & 4
\end{array}\right] \text {, } \\
& \boldsymbol{\mu}_{3}=\left[\begin{array}{ll}
8 & 3
\end{array}\right], \\
& \boldsymbol{\Sigma}_{1}=\left[\begin{array}{ll}
2 & 0 \\
0 & 3
\end{array}\right] \text {, } \\
& \boldsymbol{\Sigma}_{2}=\left[\begin{array}{ll}
2 & 0 \\
0 & 3
\end{array}\right] \text {, } \\
& \boldsymbol{\Sigma}_{3}=\left[\begin{array}{cc}
0,1 & 0 \\
0 & 0,1
\end{array}\right] \text {, } \\
& \pi_{1}=\frac{1}{3} \text {, } \\
& \pi_{2}=\frac{1}{3} \text {, } \\
& \pi_{3}=\frac{1}{3} \text {. }
\end{aligned}
$$

Assumindo que o indivíduo $\mathcal{G}$ que irá sofrer a divisão de componentes representa o modelo ilustrado na Figura 3.2(a) temos:

$$
\begin{aligned}
\boldsymbol{\mu}_{1} & =\left[\begin{array}{ll}
1 & 2,5
\end{array}\right], \\
\boldsymbol{\Sigma}_{1} & =\left[\begin{array}{ll}
2 & 0 \\
0 & 3
\end{array}\right], \\
\pi_{1} & =\frac{2}{3},
\end{aligned}
$$

$$
\begin{aligned}
\boldsymbol{\mu}_{2} & =\left[\begin{array}{ll}
8 & 3
\end{array}\right] \\
\boldsymbol{\Sigma}_{2} & =\left[\begin{array}{cc}
0,1 & 0 \\
0 & 0,1
\end{array}\right], \\
\pi_{2} & =\frac{1}{3} .
\end{aligned}
$$

Utilizando a medida $D_{L L K}$ para avaliar cada grupo, obtemos $D_{L L K}(1) \approx 7.807$ e $D_{L L K}(2) \approx$ 3.802 , enquanto que de acordo com a medida $D_{L K D}$ os valores para $C_{1}$ e $C_{2}$ são 0,71 e 0,41 , respectivamente. Conforme esperado, o grupo $C_{1}$ tem mais chances de ser escolhido para a divisão, já que o grupo $C_{2}$ modela bem uma região densa dos dados.

Considere agora que o grupo $C_{1}=\left\{\pi_{1}, \boldsymbol{\mu}_{1}, \boldsymbol{\Sigma}_{1}\right\}$ foi escolhido para ser dividido em dois grupos. Os parâmetros dos novos grupos obtidos pelo SVDS, considerando os parâmetros do 
operador $\alpha=u=\beta=0,5^{5}$, são:

(i) $\pi_{i}=0,667 \times 0,5=0,333$;

$\pi_{j}=0,667 \times 0,5=0,333$

(ii) $\boldsymbol{\mu}_{i}=\left[\begin{array}{ll}1 & 2,5\end{array}\right]-\sqrt{\frac{0,333}{0,333}} \times 0,5 \times\left[\begin{array}{ll}0 & 1,7321\end{array}\right]=\left[\begin{array}{ll}1 & 1,634\end{array}\right]$;

$\boldsymbol{\mu}_{j}=\left[\begin{array}{ll}1 & 2,5\end{array}\right]+\sqrt{\frac{0,333}{0,333}} \times 0,5 \times\left[\begin{array}{ll}0 & 1,7321\end{array}\right]=\left[\begin{array}{ll}1 & 3,366\end{array}\right] ;$

(iii) $\Sigma_{i}=\frac{0,333}{0,333} \times \operatorname{diag}^{6}\left(\left[\begin{array}{ll}2 & 3\end{array}\right]\right)+\left(0,5-0,5^{3}-1\right) \times \frac{0,667}{0,333} \times\left[\begin{array}{ll}0 & 1,732\end{array}\right] \times\left[\begin{array}{ll}0 & 1,732\end{array}\right]^{T}+$ $\left[\begin{array}{ll}0 & 1,732\end{array}\right] \times\left[\begin{array}{ll}0 & 1,732\end{array}\right]^{T}=\left[\begin{array}{cc}2 & 0 \\ 0 & 2,25\end{array}\right]$;

$\boldsymbol{\Sigma}_{j}=\frac{0,333}{0,333} \times \operatorname{diag}\left(\left[\begin{array}{ll}2 & 3\end{array}\right]\right)+\left(0,5^{3}-0,5-0,5^{2}\right) \times \frac{0,667}{0,333} \times\left[\begin{array}{ll}0 & 1,732\end{array}\right] \times\left[\begin{array}{ll}0 & 1,732\end{array}\right]^{T}+$ $\left[\begin{array}{ll}0 & 1,732\end{array}\right] \times\left[\begin{array}{ll}0 & 1,732\end{array}\right]^{T}=\left[\begin{array}{cc}2 & 0 \\ 0 & 2,25\end{array}\right]$.

É possível verificar que os novos grupos são similares aos grupos corretos e com a aplicação do operador a partição encontra-se agora mais próxima do modelo que originou os dados (Figura 3.2(b)). Neste contexto, uma nova execução do EM, iniciando-o com as distribuições gaussianas obtidas permitiria encontrar um GMM que provavelmente tenha gerado os dados em questão.

Considerando o mesmo cenário, mas com o operador VS, sendo a divisão realizada considerando o atributo 2, i.e., a $2^{\text {a }}$ coluna da matriz de covariância $\Sigma_{1}$, as médias dos novos grupos criados são: $\boldsymbol{\mu}_{i}=\left[\begin{array}{ll}1 & 2,5\end{array}\right]+\left[\begin{array}{ll}0 & 3\end{array}\right]=\left[\begin{array}{ll}1 & 5.5\end{array}\right]$ e $\boldsymbol{\mu}_{j}=\left[\begin{array}{ll}1 & 2,5\end{array}\right]-\left[\begin{array}{ll}0 & 3\end{array}\right]=\left[\begin{array}{ll}1 & -0.5\end{array}\right]$. Aplicando 5 iterações $\left(t_{k m}=5\right)$ do algoritmo $K$-means nos objetos com probabilidade maior de pertencer ao grupo $C_{1}$ os parâmetros obtidos são:

$$
\begin{aligned}
\boldsymbol{\mu}_{i} & =\left[\begin{array}{lll}
1,02 & 3,99
\end{array}\right], & \boldsymbol{\mu}_{j} & =\left[\begin{array}{ll}
0,94 & 0,96
\end{array}\right] \\
\boldsymbol{\Sigma}_{i} & =\left[\begin{array}{ll}
1,9387 & 0,0543 \\
0,0543 & 0,2796
\end{array}\right], & \boldsymbol{\Sigma}_{j} & =\left[\begin{array}{cc}
2,0574 & -0,0302 \\
-0,0302 & 0,3080
\end{array}\right], \\
\pi_{i} & =\frac{500}{1000} \times \frac{2}{3}=\frac{1}{3}, & \pi_{j} & =\frac{500}{1000} \times \frac{2}{3}=\frac{1}{3} .
\end{aligned}
$$

Na Figura 3.2(c) pode ser visualizado o modelo após a aplicação do operador. Diferentemente do modelo resultante do SVDS, após a aplicação do VS os parâmetros já estão melhor ajustados aos dados devido às iterações do $K$-means.

\footnotetext{
${ }^{5}$ Valores sugeridos por Zhang et al. (2003).

${ }^{6} \mathrm{~A}$ função diag representa os valores da diagonal principal de uma matriz na qual apenas os valores nessa diagonal são não-zeros.
} 


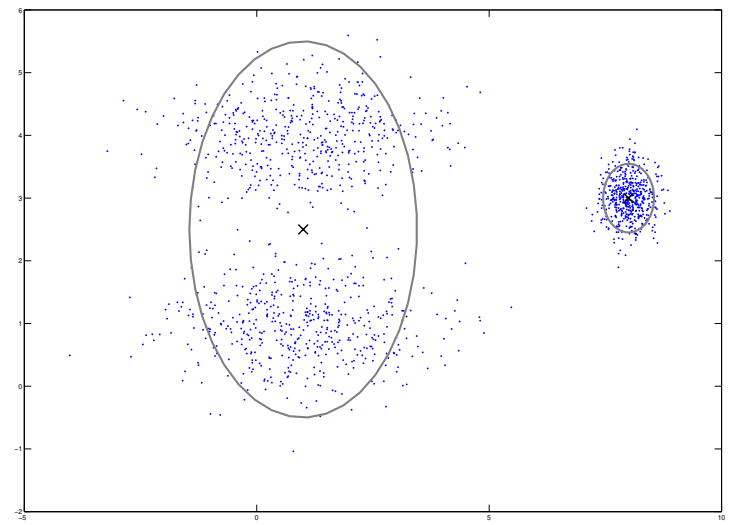

(a) Antes da aplicação do operador.

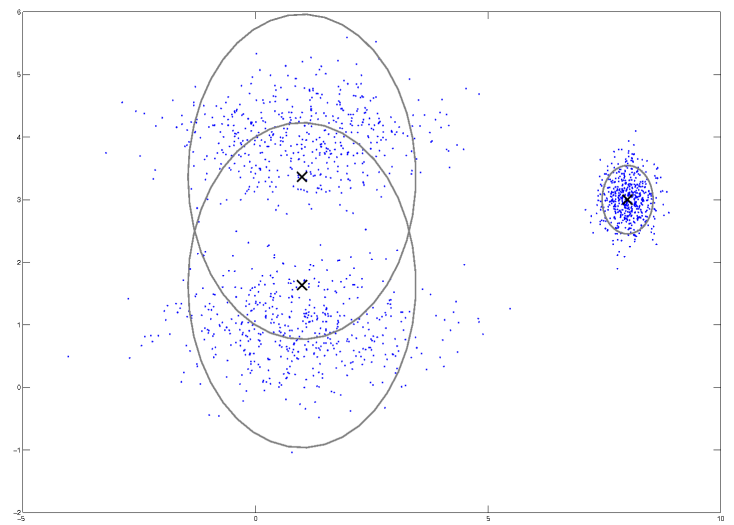

(b) Após a aplicação do operador SVDS.

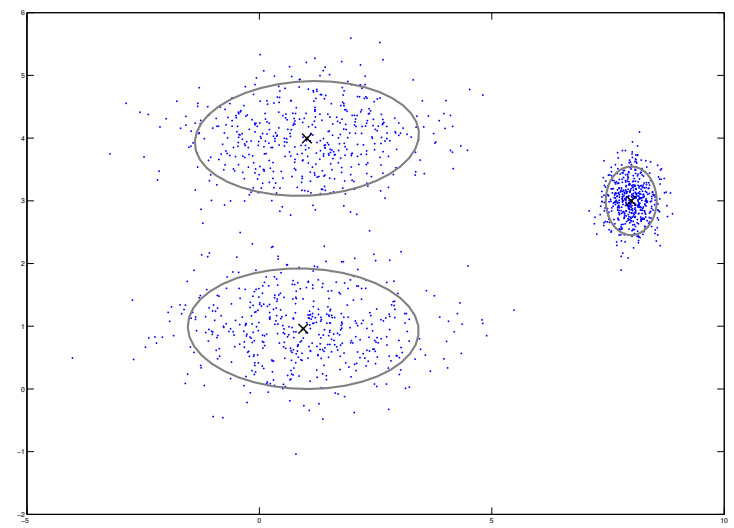

(c) Após a aplicação do operador VS.

Figura 3.2: Exemplo da aplicação do operadores de divisão. 


\subsubsection{Resumo de Variantes}

O uso das diferentes medidas de avaliação para união e divisão de componentes, bem como dos diferentes operadores de divisão e união, dão origem a um conjunto de variantes do ESM-EM. Para se referir a estas variantes, é utilizada uma combinação dos acrônimos dos seus respectivos operadores/medidas. Por exemplo, VS-OPER-PLL se refere ao algoritmo obtido usando o operador Variance based Splitting (VS), a medida de overlap (Equação (3.3) para indicar quais grupos devem ser unidos, e a parcela de contribuição para a função de verossimilhança (Equação (3.1)) para avaliar qual grupo deve ser dividido. Um sumário com todas as variantes é fornecido na Tabela 3.1. Essas diferentes variantes são comparadas empiricamente na Seção 3.4.1.

Tabela 3.1: Sumário das variantes desenvolvidas

\begin{tabular}{cccc}
\hline Nome & Operador de divisão & Medida de divisão & Medida de união \\
\hline SVD & SVDS - Alg. 9 & LKD - Eq. (2.15) & Correlação - Eq. (2.8) \\
OVER & SVDS - Alg. 9 & LKD - Eq. (2.15) & Sobreposição - Eq. (3.3) \\
VS & VS - Alg. 10 & LKD - Eq. (2.15) & Correlação - Eq. (2.8) \\
PLL & SVDS - Alg. 9 & PLL - Eq. (3.1) & Correlação - Eq. (2.8) \\
VS-OVER & VS - Alg. 10 & LKD - Eq. (2.15) & Sobreposição - Eq. (3.3) \\
VS-PLL & VS - Alg. 10 & PLL - Eq. (3.1) & Correlação - Eq. (2.8) \\
OVER-PLL & SVDS - Alg. 9 & PLL - Eq. (3.1) & Sobreposição - Eq. (3.3) \\
VS-OVER-PLL & VS - Alg. 10 & PLL - Eq. (3.1) & Sobreposição - Eq. (3.3) \\
\hline
\end{tabular}

\subsection{Notas sobre Complexidade Computacional}

Como esperado, a complexidade computacional do ESM-EM depende da escolha dos operadores discutidos. No entanto, o custo dominante do ESM-EM consiste na aplicação do algoritmo EM. Por esta perspectiva, o custo computacional do EM pode ser estimado como: $O\left(G \cdot P\left(N \cdot k_{\max } \cdot M^{3} \cdot t_{E M}+N \cdot K_{\max }^{2}\right)\right)$, sendo $G$ o número de gerações e $t_{E M}$ o número de iterações do EM executadas em cada passo de refinamento. O último termo aparece da computação das probabilidades de se unir cada par de grupos. Este custo é o mesmo do algoritmo GA-EM (Pernkopf e Bouchaffra, 2005) e similar ao do GAKREM ${ }^{7}$ (Nguyen e Cios, 2008). Assumindo que o usuário não possui conhecimento a priori sobre uma faixa de números de grupos de interesse, cenário em que AE são mais aplicados, a heurística comumente de $K_{\max }=\sqrt{N}$ pode ser aplicada (Campello et al., 2009). Neste caso, o custo computacional pode ser simplificado para $O\left(G \cdot P\left(N^{1.5} \cdot M^{3}+N^{2}\right)\right)$, pois $t_{E M} \ll N$. Apesar deste custo ser maior do que o custo do OMR-EM (ver Seção 2.2), a Seção 3.4.2 apresenta evidências empíricas de que o custo adicional (em termos de complexidade assintótica) por iteração, necessário para guiar a busca para melhores modelos, de fato provê melhores modelos em menos tempo.

\footnotetext{
${ }^{7}$ GAKREM possui um custo de $O\left(G \cdot K_{\max } \cdot N \cdot M^{3}\right)$ quando o algoritmo EM é utilizado com matrizes de covariâncias irrestritas.
} 


\subsection{Avaliação Empírica}

Para analisar a eficiência e acurácia dos algoritmos propostos, foram consideradas 35 bases de dados. Três delas são conhecidas na literatura, especificamente: (i) 3-componentes (Ueda e Nakano, 1998), formada por 900 objetos distribuídos em três componentes gaussianos bidimensionais; (ii) 9Gauss (Campello et al., 2009), que possui 900 objetos de nove componentes gaussianos bi-dimensionais com sobreposição, e (iii) Pendigits, que é uma base de dados de classificação composta por imagens de dígitos escritos à mão (Asuncion e Newman, 2007). Para a última, foram consideradas apenas as classes representando os dígitos $0,1,2,3$, e 4, tendo, portanto, 5.629 objetos. Similarmente ao realizado por Pernkopf e Bouchaffra (2005), foi aplicada a técnica de Análise de Componentes Principais (PCA, do inglês Principal Component Analisys) (Bishop, 2006), reduzindo de 16 para 2 o número de atributos. Também foi considerada uma versão estendida da base de dados 9Gauss (Campello et al., 2009), chamada de 49Gauss, que possui áreas de sobreposição similares à 9Gauss, mas com 49 Gaussianas e 4.900 objetos. Além destas bases de dados, foram conduzidos experimentos com outras 7 bases que podem ser modeladas por meio de GMMs, embora não sejam geradas por GMMs de fato. Figura 3.3 ilustra essas bases de dados. As 24 bases de dados restantes são bases sintéticas geradas de acordo com dois critérios de separação de componentes da literatura. Critérios de separação de componentes são comumente utilizados para: (i) servir de fundamentos para provas teóricas sobre convergência de algoritmos em situações de complexidade da base de dados pré-definidas (Dasgupta, 1999); e (ii) gerar bases de dados de diferentes complexidades para análises empíricas (Maitra e Melnykov, 2010). Nesta tese, utilizamos tais critérios com a motivação (ii). Os dois critérios de separação de componentes considerados foram:

- c-separation (Dasgupta, 1999): Distribuições gaussianas $\mathcal{N}\left(\boldsymbol{\mu}_{i}, \boldsymbol{\Sigma}_{i}\right)$ e $\mathcal{N}\left(\boldsymbol{\mu}_{j}, \boldsymbol{\Sigma}_{j}\right)$ são $c$ separadas se:

$$
\left\|\boldsymbol{\mu}_{i}-\boldsymbol{\mu}_{j}\right\| \geq c \sqrt{M \cdot \max \left(\lambda_{\max }\left(\boldsymbol{\Sigma}_{i}\right), \lambda_{\max }\left(\boldsymbol{\Sigma}_{j}\right)\right)}
$$

sendo $\lambda_{\max }\left(\Sigma_{i}\right)$ o maior autovalor de $\Sigma_{i}$.

- $\omega$-separation (Maitra e Melnykov, 2010): soma das probabilidade de classificação incorreta entre duas Gaussianas $\mathcal{N}\left(\boldsymbol{\mu}_{i}, \boldsymbol{\Sigma}_{i}\right)$ e $\mathcal{N}\left(\boldsymbol{\mu}_{j}, \boldsymbol{\Sigma}_{j}\right)$ de um GMM:

$$
\begin{aligned}
\tau_{i}(\mathbf{x}) & =\pi_{i} \mathcal{N}\left(\mathbf{x} \mid \boldsymbol{\mu}_{i}, \boldsymbol{\Sigma}_{i}\right), \\
\tau_{j}(\mathbf{x}) & =\pi_{j} \mathcal{N}\left(\mathbf{x} \mid \boldsymbol{\mu}_{j}, \boldsymbol{\Sigma}_{j}\right), \\
\omega_{i \mid j} & =\operatorname{Pr}\left[\tau_{j}(\mathbf{x})<\tau_{i}(\mathbf{x}) \mid \mathbf{x} \sim \mathcal{N}\left(\mathbf{x} \mid \boldsymbol{\mu}_{j}, \boldsymbol{\Sigma}_{j}\right)\right], \\
\omega_{j \mid i} & =\operatorname{Pr}\left[\tau_{i}(\mathbf{x})<\tau_{j}(\mathbf{x}) \mid \mathbf{x} \sim \mathcal{N}\left(\mathbf{x} \mid \boldsymbol{\mu}_{i}, \boldsymbol{\Sigma}_{i}\right)\right], \\
\omega_{i j} & =\omega_{i \mid j}+\omega_{j \mid i} .
\end{aligned}
$$




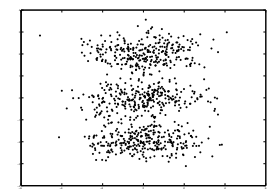

(a) componentes (Ueda e Nakano, 1998)

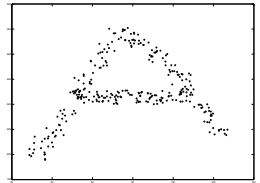

(e) Letra A

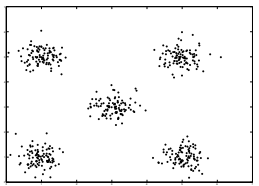

(i) $\mathrm{Em} \mathrm{X}$

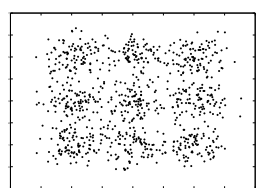

(b) (Campello et al., 2009)

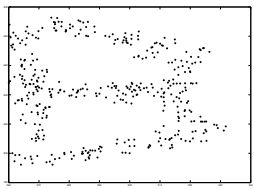

(f) Letra B

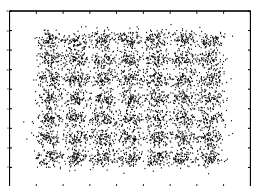

(c) 49Gauss

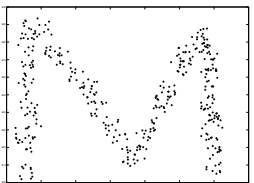

(g) Letra M

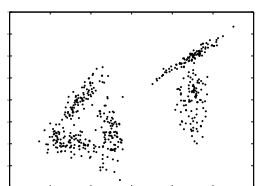

(d) Triângulo

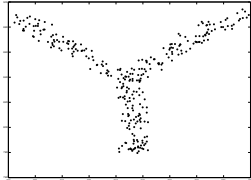

(h) Letra Y

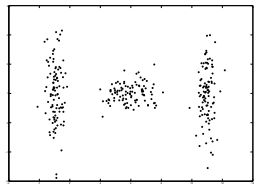

(j) Duas barras

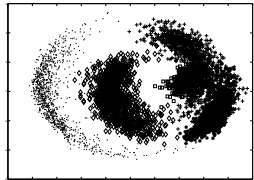

(k) Pendigits (Asuncion e Newman, 2007)

Figura 3.3: Gráfico de dispersão de bases de dados utilizadas nos experimentos.

Um sumário das principais características das bases de dados utilizadas nas comparações é fornecido na Tabela 3.2. Uma ressalva importante em relação ao critério $c$-separation é que ele atua como um limite inferior da complexidade da base de dados. Portanto, um GMM 0,5separado ${ }^{8}$ pode ser menos complexo que um GMM 2-separado. Para suavizar isso, usamos a abordagem descrita por (Maitra, 2009), em que os componentes são tais que pelo menos um par tem separação entre $c$ e $c+0,005$. A Figura 3.4 mostra um exemplo de valores de separação para pares de componentes gaussianas de um GMM de acordo com os critérios $c$-separation e $\omega$ separation. Foram usados três valores diferentes para cada critério. Mais especificamente, para o critério $c$-separation foram utilizados $c \in\left\{5 \cdot 10^{-1}, 1,2\right\}$ e $\bar{\omega}=\left\{10^{-1}, 10^{-2}, 10^{-3}\right\}$, sendo $\bar{\omega}$ a média de $\omega_{i j}$ para todos os pares de componentes $i \neq j$. Para cada nível de complexidade, foram geradas bases de dados com $K=\{4,12\}$ e $M=\{4,8\}$ e $10^{5}$ amostras (objetos). A Tabela 3.3 mostra os valores de separação para cada base de dados gerada. Este conjunto de base de dados artificiais constitui-se de configurações conhecidas como bem-separadas e malseparadas conforme discutido em Dasgupta (1999) e Maitra e Melnykov (2010).

As análises estão divididas em duas partes. Inicialmente, na Seção 3.4.1, as diferentes variantes do algoritmo ESM-EM discutidas na Seção 3.2 são comparadas. Considerando os resultados obtidos nestas análises, a melhor variante do ESM-EM foi selecionada e comparada com o algoritmo evolutivo estado-da-arte proposto por Pernkopf e Bouchaffra (2005). De forma a se

\footnotetext{
${ }^{8}$ Um GMM $c$-separado é um em que todos os pares de componentes gaussianos satisfazem o critério de $c$ separation.
} 
Tabela 3.2: Sumário das bases de dados. Número correto de grupos $(K)$ para a base de dados Pendigits não é conhecido.

\begin{tabular}{cccc|cccc}
\hline \hline Base de dados & $N$ & $M$ & $K$ & Base de dados & $N$ & $M$ & $K$ \\
\hline 9Gauss & 900 & 2 & 9 & $c 1.0-\mathrm{k} 12 \mathrm{M} 4$ & 1,200 & 4 & 12 \\
49Gauss & 4,900 & 2 & 49 & $c 2.0-\mathrm{k} 12 \mathrm{M} 4$ & 1,200 & 4 & 12 \\
3-componentes & 900 & 2 & 3 & $c 0.5-\mathrm{k} 12 \mathrm{M} 8$ & 1,200 & 8 & 12 \\
Triângulo & 500 & 2 & 5 & $c 1.0-\mathrm{k} 12 \mathrm{M} 8$ & 1,200 & 8 & 12 \\
Letra A & 300 & 2 & 3 & $c 2.0-\mathrm{k} 12 \mathrm{M} 8$ & 1,200 & 8 & 12 \\
Letra B & 400 & 2 & 5 & $\omega 0.1-\mathrm{k} 4 \mathrm{M} 4$ & 400 & 4 & 4 \\
Letra M & 400 & 2 & 4 & $\omega 0.01-\mathrm{k} 4 \mathrm{M} 4$ & 400 & 4 & 4 \\
Letra Y & 300 & 2 & 3 & $\omega 0.001-\mathrm{k} 4 \mathrm{M} 4$ & 400 & 4 & 4 \\
Em X & 500 & 2 & 5 & $\omega 0.1-\mathrm{k} 4 \mathrm{M} 8$ & 400 & 8 & 4 \\
Duas barras & 300 & 2 & 3 & $\omega 0.01-\mathrm{k} 4 \mathrm{M} 8$ & 400 & 8 & 4 \\
Pendigits & 5,629 & 2 & - & $\omega 0.001-\mathrm{k} 4 \mathrm{M} 8$ & 400 & 8 & 4 \\
$c 0.5-\mathrm{k} 4 \mathrm{M} 4$ & 400 & 4 & 4 & $\omega 0.1-\mathrm{k} 12 \mathrm{M} 4$ & 1,200 & 4 & 12 \\
$c 1.0-\mathrm{k} 4 \mathrm{M} 4$ & 400 & 4 & 4 & $\omega 0.01-\mathrm{k} 12 \mathrm{M} 4$ & 1,200 & 4 & 12 \\
$c 2.0-\mathrm{k} 4 \mathrm{M} 4$ & 400 & 4 & 4 & $\omega 0.001-\mathrm{k} 12 \mathrm{M} 4$ & 1,200 & 4 & 12 \\
$c 0.5-\mathrm{k} 4 \mathrm{M} 8$ & 400 & 8 & 4 & $\omega 0.1-\mathrm{k} 12 \mathrm{M} 8$ & 1,200 & 8 & 12 \\
$c 1.0-\mathrm{k} 4 \mathrm{M} 8$ & 400 & 8 & 4 & $\omega 0.01-\mathrm{k} 12 \mathrm{M} 8$ & 1,200 & 8 & 12 \\
$c 2.0-\mathrm{k} 4 \mathrm{M} 8$ & 400 & 8 & 4 & $\omega 0.001-\mathrm{k} 12 \mathrm{M} 8$ & 1,200 & 8 & 12 \\
$c 0.5-\mathrm{k} 12 \mathrm{M} 4$ & 1,200 & 4 & 12 & & & & \\
\hline
\end{tabular}

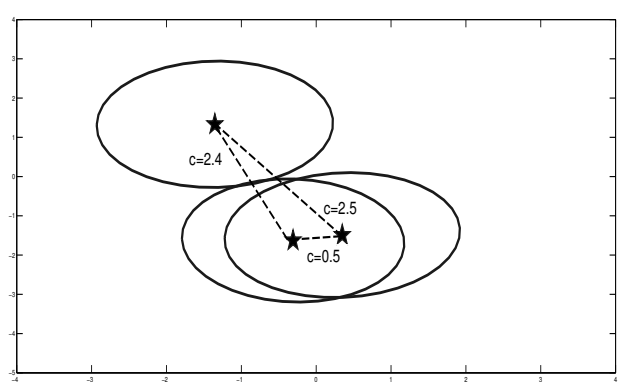

(a)

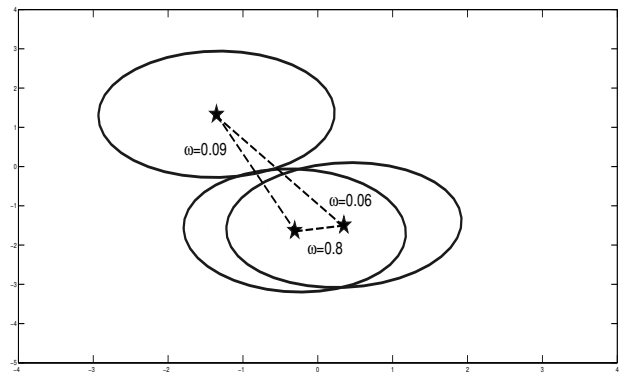

(b)

Figura 3.4: Exemplo dos critérios de separação para cada par de componentes gaussianas de um GMM: (a) c-separation; (b) w-separation.

ter um método baseline de comparação, nesta análise também é considerada o uso do Ordered Multiple Runs of Expectation Maximization (OMR-EM) (Algoritmo 1). Estas comparações são apresentadas na Seção 3.4.2.

\subsubsection{Comparação entre Variantes do ESM-EM}

Nesta seção são, reportadas os valores médios obtidos após 10 execuções de cada variante do ESM-EM em cada base de dados. Os parâmetros das variantes foram definidos da seguinte forma: (i) critério de parada $\left(S_{C}\right)$ definido como no máximo 50 gerações ou até 10 gerações consecutivas sem melhoras no melhor indivíduo; (ii) o tamanho da população $(P)$ igual a 10; (iii) número máximo de iterações do $\mathrm{EM}\left(t_{E M}\right)$ igual a 5; (iv) número máximo de iterações do $K$-means ( $t_{k m}$ para o operador VS) igual a $5 ;(\mathrm{v}) \alpha, u, \beta$ no operador SVDS igual a 0,5 (mesmos valores usados em Zhang et al. (2003)); (vi) número máximo de grupos $\left(K_{\max }\right)$ igual a $\sqrt{N}$. Os resultados foram sumarizados utilizando três critérios: (i) valor da função objetivo (MDL); (ii) número de iterações do EM realizadas durante a busca evolutiva; (iii) acurácia na estimação do 
Tabela 3.3: Valores de critérios de separação $c$ e $\omega$ para os dados gerados.

\begin{tabular}{cccccc}
\hline Base de dados & $\min (\mathbf{c})$ & $\operatorname{média}(\mathbf{c})$ & $\max (\mathbf{c})$ & $\operatorname{média}(\omega)$ & $\max (\omega)$ \\
\hline$c 0,5-\mathrm{k} 4 \mathrm{M} 4$ & 0,50 & 1,90 & 2,80 & 0,117 & 0,554 \\
$c 1,0-\mathrm{k} 4 \mathrm{M} 4$ & 1,00 & 2,70 & 3,30 & 0,035 & 0,206 \\
$c 2,0-\mathrm{k} 4 \mathrm{M} 4$ & 2,00 & 2,80 & 3,90 & 0,006 & 0,021 \\
$c 0,5-\mathrm{k} 4 \mathrm{M} 8$ & 0,50 & 1,40 & 1,80 & 0,055 & 0,311 \\
$c 1,0-\mathrm{k} 4 \mathrm{M} 8$ & 1,00 & 1,90 & 2,20 & 0,013 & 0,077 \\
$c 2,0-\mathrm{k} 4 \mathrm{M} 8$ & 2,00 & 3,40 & 4,00 & 0,000 & 0,000 \\
$c 0,5-\mathrm{k} 12 \mathrm{M} 4$ & 0,50 & 2,90 & 4,00 & 0,017 & 0,549 \\
$c 1,0-\mathrm{k} 12 \mathrm{M} 4$ & 1,00 & 2,90 & 4,00 & 0,014 & 0,236 \\
$c 2,0-\mathrm{k} 12 \mathrm{M} 4$ & 2,00 & 2,90 & 4,00 & 0,004 & 0,022 \\
$c 0,5-\mathrm{k} 12 \mathrm{M} 8$ & 0,50 & 2,10 & 4,00 & 0,007 & 0,312 \\
$c 1,0-\mathrm{k} 12 \mathrm{M} 8$ & 1,00 & 3,10 & 4,00 & 0,001 & 0,061 \\
$c 2,0-\mathrm{k} 12 \mathrm{M} 8$ & 2,00 & 3,20 & 4,00 & 0,000 & 0,000 \\
$\omega 0,1-\mathrm{k} 4 \mathrm{M} 4$ & 0,60 & 1,10 & 1,50 & 0,100 & 0,239 \\
$\omega 0,01-\mathrm{k} 4 \mathrm{M} 4$ & 1,20 & 1,50 & 2,10 & 0,010 & 0,023 \\
$\omega 0,001-\mathrm{k} 4 \mathrm{M} 4$ & 1,70 & 1,90 & 2,10 & 0,001 & 0,003 \\
$\omega 0,1-\mathrm{k} 4 \mathrm{M} 8$ & 0,40 & 0,70 & 0,90 & 0,100 & 0,241 \\
$\omega 0,01-\mathrm{k} 4 \mathrm{M} 8$ & 0,90 & 1,20 & 1,60 & 0,010 & 0,036 \\
$\omega 0,001-\mathrm{k} 4 \mathrm{M} 8$ & 1,20 & 1,60 & 2,00 & 0,001 & 0,005 \\
$\omega 0,1-\mathrm{k} 12 \mathrm{M} 4$ & 0,10 & 1,00 & 1,90 & 0,100 & 0,502 \\
$\omega 0,01-\mathrm{k} 12 \mathrm{M} 4$ & 0,70 & 2,40 & 5,50 & 0,010 & 0,176 \\
$\omega 0,001-\mathrm{k} 12 \mathrm{M} 4$ & 1,10 & 5,50 & 10,10 & 0,001 & 0,065 \\
$\omega 0,1-\mathrm{k} 12 \mathrm{M} 8$ & 0,30 & 0,70 & 1,00 & 0,100 & 0,300 \\
$\omega 0,01-\mathrm{k} 12 \mathrm{M} 8$ & 0,60 & 1,30 & 2,10 & 0,010 & 0,102 \\
$\omega 0,001-\mathrm{k} 12 \mathrm{M} 8$ & 0,80 & 1,80 & 2,60 & 0,001 & 0,020 \\
\hline
\end{tabular}

número de grupos. Para cada critério foi gerado um ranking dos algoritmos de acordo com os valores obtidos por cada algoritmo em cada base de dados, e na sequência o ranking médio de cada algoritmo é obtido. Por exemplo, se um algoritmo apresentou os melhores resultados de acordo com o critério MDL em todas as bases de dados, seu ranking médio é 1. A Tabela 3.4 apresenta as diferenças entre os ranks médios obtidos por cada variante. Considerando o critério MDL, o uso do operador Variance based Splitting (VS) e a função Partial Log-Likelihood (PLL) — variante VS-PLL — apresentou melhores resultados consistentemente, i.e., com menor ranking médio. Como este é o principal critério em termos de qualidade na estimação dos parâmetros, esta é a variante recomendada por este estudo, que também é o método recomendado se considerado um tradeoff ${ }^{9}$ entre os três critérios.

Tabela 3.4: Ranking médio de cada variante considerando o valor de adequabilidade (MDL), número de iterações do EM (EMS), diferença absoluta entre número de grupos predito real $(\approx$ $\mathrm{K})$. A última coluna apresenta a média dos rankings de cada algoritmo.

\begin{tabular}{ccccc}
\hline Variante & MDL & EMS & $\approx \mathbf{K}$ & Ranking médio \\
\hline SVD & 5,03 & $\mathbf{3 , 5 7}$ & 4,99 & 4,53 \\
OVER & 4,50 & 4,40 & 4,79 & 4,56 \\
VS & 4,63 & 4,77 & 5,20 & 4,87 \\
PLL & 4,31 & 4,94 & 4,16 & 4,47 \\
VS-OVER & 4,56 & 4,39 & 4,61 & 4,52 \\
VS-PLL & $\mathbf{4 , 0 1}$ & 3,83 & 3,84 & $\mathbf{3 , 9 0}$ \\
OVER-PLL & 4,36 & 4,74 & 4,86 & 4,65 \\
VS-OVER-PLL & 4,60 & 5,36 & $\mathbf{3 , 5 6}$ & 4,50 \\
\hline
\end{tabular}

Após apresentar tais observações gerais, pode-se avaliar os algoritmos considerando seus resultados nas bases de dados complexas. Para avaliar a qualidade dos GMMs obtidos pelas

\footnotetext{
${ }^{9}$ Considerando o ranking médio dos três critérios.
} 

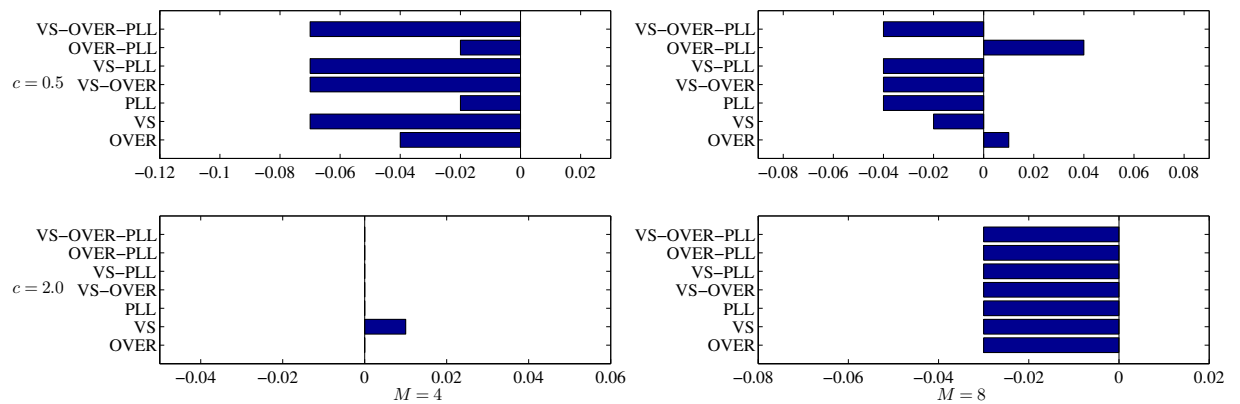

Figura 3.5: Diferenças médias de HLL obtidas pelas diferentes variantes do ESM-EM e a variante SVD nas bases de dados $c$-separation com $K=4$ (quanto menor o valor melhor a variante). Resultados para as bases de dados $\operatorname{com} c=1$ foram omitidos devido ao fato de que nenhuma diferença foi observada.

diversas variantes, foi considerada a verossimilhança em uma amostra, não utilizada antes, de $10^{4}$ objetos gerados por meio do GMM gerador dos dados utilizados previamente. Por conveniência, denomina-se o valor obtido de Held-out Log-Likelihood (HLL). As Figuras 3.5-3.8 mostram as diferenças médias (por objeto) obtidas de HLL entre cada variante e a variante SVD que foi escolhida para servir de baseline para comparação. Portanto, quanto menor o valor apresentado, melhor a variante.

Algumas observações podem ser feitas a partir das Figuras 3.5-3.8. Quando o número de atributos e grupos é pequeno $(M=K=4)$, não são observadas diferenças entre os modelos obtidos por cada variante, exceto no caso de $c=0,5$. Isto pode ser explicado por dois fatores: (i) o espaço de busca não possui muitos ótimos locais e, portanto, quaisquer operadores são capazes de chegar a mesma (melhor) solução; (ii) os dados gerados para $c=0,5$ são muito mais complexos que os outros por causa da existência de um par de componentes com grande sobreposição (como pode ser visto no valor de $\max (\omega)$ na Tabela 3.3). Portanto, é mais provável a existência de um grande número de mínimos locais. Considerando um número maior de atributos $(M=8, K=4)$, as diferenças entre as variantes aparecem com maior frequência e todas as variantes são capazes de obter um modelo melhor que SVD em pelo menos 5 das 6 bases de dados. No cenário oposto (maior número de grupos $M=4, K=12$ ), as diferenças entre os modelos são geralmente insignificantes (valor de log-verossimilhança menores que 0,01). É evidente nestes resultados que a maioria das variantes foram capazes de chegar a mesma (melhor) solução, exceto pela SVD. Tal observação sugere que, em tais casos, escolher uma variante mais simples (eficiente) seria suficiente para ter boas chances de escapar de ótimos locais. No cenário mais interessante, que envolve número alto de atributos e grupos $(M=$ $8, K=12$ ), isto não parece se sustentar. Especificamente, a variante VS-PLL obteve modelos iguais ou melhores que as demais variantes em todas as bases de dados, conforme discutido anteriormente. Considerando todos as evidências empíricas, conclui-se que, entre as diferentes variantes do ESM-EM analisadas, a VS-PLL é a mais promissora e, portanto, nas comparações realizadas nas seções subsequentes ela é utilizada. 

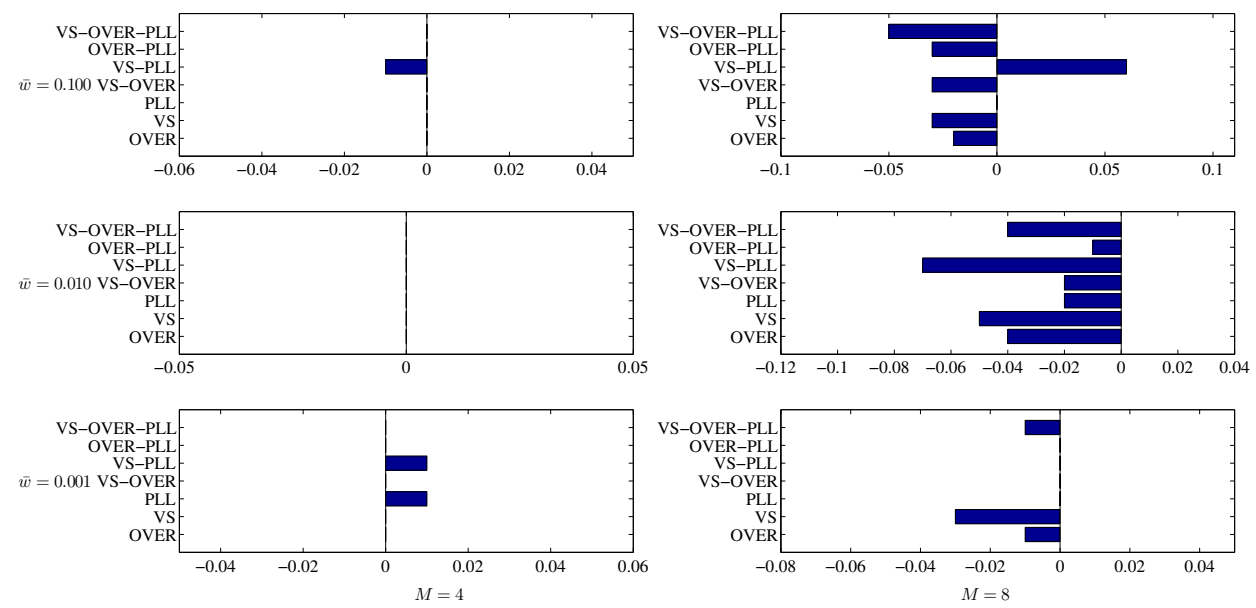

Figura 3.6: Diferenças médias de HLL obtidas pelas diferentes variantes do ESM-EM e a variante SVD nas bases de dados $\omega$-separation com $K=4$ (quanto menor o valor melhor a variante).
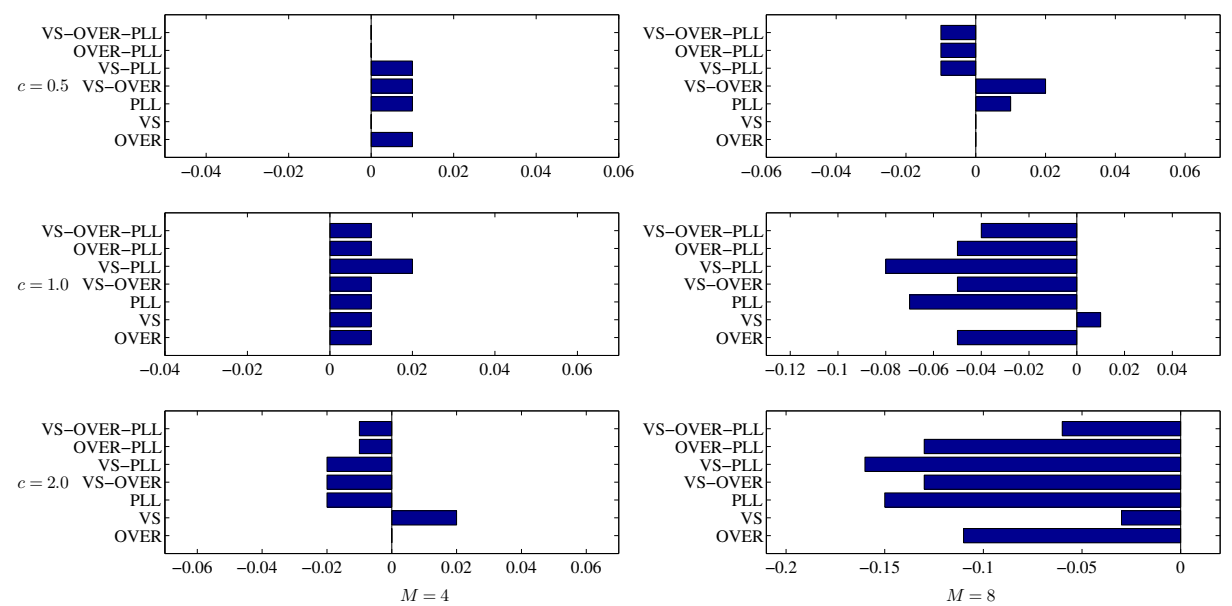

Figura 3.7: Diferenças médias de HLL obtidas pelas diferentes variantes do ESM-EM e a variante SVD nas bases de dados $c$-separation com $K=12$ (quanto menor o valor melhor a variante). 

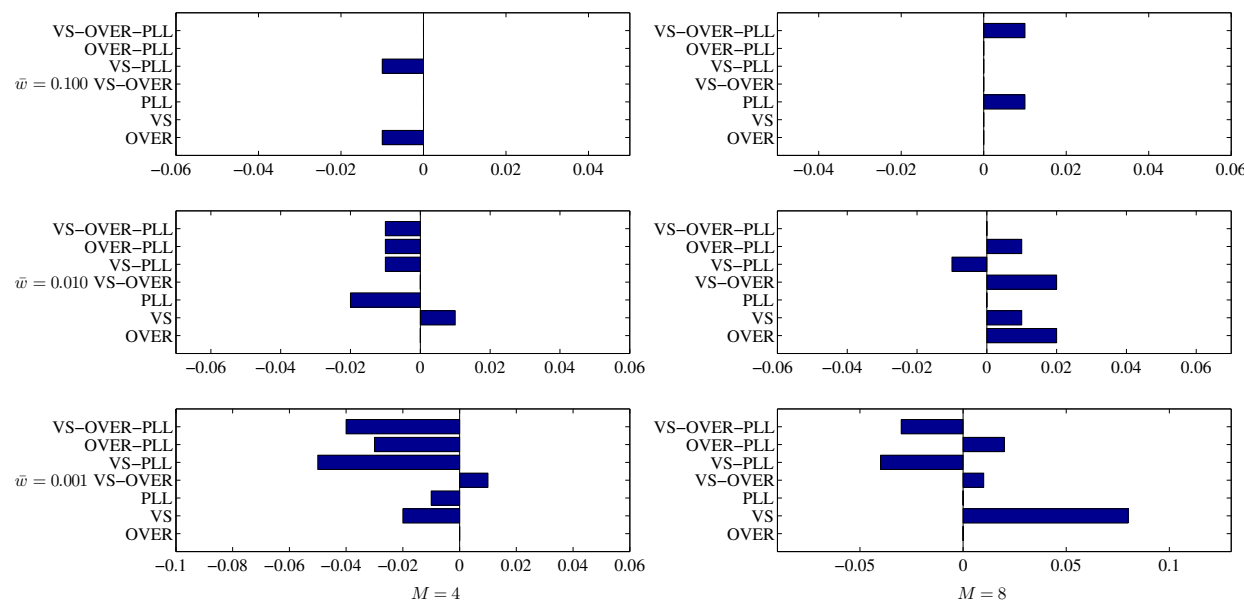

Figura 3.8: Diferenças médias de HLL obtidas pelas diferentes variantes do ESM-EM e a variante SVD nas bases de dados $\omega$-separation com $K=12$ (quanto menor o valor melhor a variante).

\subsubsection{Comparação entre ESM-EM, GA-EM e OMR-EM}

Nesta seção, a melhor variante do ESM-EM, VS-PLL, é comparada com o algoritmo evolutivo estado-da-arte Genetic-based Expectation Maximization (GA-EM) (Pernkopf e Bouchaffra, 2005). Por simplicidade, daqui em diante esta variante será referenciada apenas por ESM-EM. Nas comparações, foi utilizado o algoritmo OMR-EM (Seção 2.2) como baseline, devido ao seu amplo uso na prática. Alguns dos parâmetros do GA-EM são comuns aos de outros AEs, como as probabilidades de mutação e crossover. Junto com tais parâmetros, GA-EM possui parâmetros adicionais, como o limiar de correlação máxima e o valor mínimo de verossimilhança que um componente gaussiano pode ter. Estes parâmetros são difíceis de serem ajustados na prática, especialmente quando se usa AEs para problemas de agrupamento de dados. Dentro de experimentos controlados, no entanto, pode-se adotar um procedimento de ajuste fino dos parâmetros para se obter bons valores para cada parâmetro e seguir com as comparações. Neste sentido, foi utilizado o framework SPOT (do inglês, Sequential Parameter Optimization Toolbox) (Bartz-Beielstein et al., 2005) para otimizar os parâmetros do GA-EM. Para simplificar a análise de resultados, apenas os melhores resultados do GA-EM obtidos são discutidos na sequência. No entanto, é importante considerar que tais resultados foram obtidos após um esforço significante na otimização de seus parâmetros, exigindo custo computacional considerável para cada base de dados. Este procedimento (custoso computacionalmente) de otimização de parâmetros dificilmente poderia ser realizado em aplicações práticas.

O algoritmo ESM-EM, por sua vez, não possui parâmetros críticos. Todos os seus parâmetros (i.e., tamanho da população, número máximo de iterações do EM e $K$-means, e número máximo de grupos) podem ser definidos de acordo com os recursos computacionais disponíveis. Por exemplo, com populações menores é esperado que o algoritmo necessite de mais tempo para convergir (Naldi et al., 2011). Similarmente, o número de iterações do EM/K-means pode ser 
definido de acordo com o tempo disponível para execução. Em relação ao número máximo de grupos, o usuário pode ter as suas expectativas, e consequentemente, definir tal valor de acordo com as mesmas. Caso este não seja o caso, e assumindo que o menor número de grupos é dois, é esperado que quanto maior o número de grupos, maior o tempo de execução para explorar o espaço de busca. Finalmente, o usuário pode definir uma faixa de valores para $K$ considerando a limitação de seus recursos computacionais ou suas expectativas em relação a partição ser induzida. Neste sentido, o ESM-EM pode ser visto como uma melhoria do GA-EM, por não necessitar de otimização de seus parâmetros no processo de aprendizado, mas apenas sobre os recursos computacionais disponíveis. Sob o ponto de vista teórico, devido aos operadores do GA-EM serem guiados e baseados em operadores de divisão/união, ESM-EM pode ser visto como um algoritmo mais principiado em comparação com o GA-EM, que adota uma busca mais aleatória por meio de seus operadores.

Os algoritmos ESM-EM, GA-EM e OMR-EM foram avaliados considerando o compromisso entre qualidade do modelo obtido e tempo de execução. Para realizar uma comparação justa, todos os algoritmos foram implementados em MATLAB e executados no mesmo computador (Opteron 2GHz, 24Gb de RAM) executando apenas o sistema operacional em paralelo.

Um aspecto prático relevante a ser ilustrado é que o uso de AEs para otimizar parâmetros de um GMM, incluindo a estimação da estrutura do modelo, pode ser computacionalmente mais eficiente e eficaz do que o uso de múltiplas repetições do EM. A literatura não apresenta uma avaliação apropriada de tal aspecto. Para fazer esta análise, OMR-EM foi executado com $n_{p}=10$ e o tempo de execução de cada passagem completa sobre o número de grupos foi guardado, bem como o melhor modelo encontrado até o momento. Na sequência, os AEs (ESM-EM e GA-EM) são executados até o tempo de execução deles seja igual ao necessário pelo OMR-EM (com $n_{p}=10$ ) — armazenando as soluções intermediárias. Desta forma, as melhores soluções de cada algoritmo sob determinada restrição de tempo - considerando o tempo gasto pelo OMR-EM para diferentes valores de $n_{p}$ — podem ser comparadas. Em outras palavras, $n_{p}$ é utilizado como unidade de tempo. O tempo gasto na otimização de parâmetros do GA-EM não é considerado no tempo de execução reportado. Neste sentido, os resultados do GA-EM podem ser considerados otimistas.

A Tabela 3.5 sumariza o desempenho geral de cada algoritmo por meio dos ranking médios - considerando 10 repetições para todas as bases de dados. Por exemplo, o menor valor obtido pelo GA-EM para $n_{p}=1\left(1,47\right.$ na $2^{\mathrm{a}}$ linha e $4^{\mathrm{a}}$ coluna $)$ indica que, computando a média sobre todas as bases de dados, o algoritmo GA-EM foi capaz de obter o menor valor de MDL para cada restrição de tempo. Por meio destes resultados, é possível verificar que existe um ranking consistente dos três algoritmos para $n_{p} \leq 7$ : GA-EM, ESM-EM, e OMR-EM. Para $n_{p}>7$, ESM-EM obtém o melhor ranking. Também é possível verificar que os AEs podem ser mais computacionalmente eficientes do que múltiplas repetições do EM. Finalmente, o algoritmo ESM-EM, que não possui parâmetros críticos, é competitivo com o GA-EM mesmo quando os parâmetros do GA-EM foram otimizados a priori. Conforme discutido anteriormente, tal 
Tabela 3.5: Ranking médio obtido por cada algoritmo - considerando o valor de MDL obtido pelo melhor modelo para cada valor de $n_{p}$.

\begin{tabular}{cccc}
\hline$n_{p}$ & OMR-EM & ESM-EM & GAEM \\
\hline 1 & 2,51 & 2,01 & $\mathbf{1 , 4 7}$ \\
2 & 2,51 & 1,83 & $\mathbf{1 , 6 6}$ \\
3 & 2,46 & 1,89 & $\mathbf{1 , 6 6}$ \\
4 & 2,41 & 1,90 & $\mathbf{1 , 6 9}$ \\
5 & 2,30 & 1,93 & $\mathbf{1 , 7 7}$ \\
6 & 2,24 & 1,94 & $\mathbf{1 , 8 1}$ \\
7 & 2,16 & 1,94 & $\mathbf{1 , 9 0}$ \\
8 & 2,14 & $\mathbf{1 , 8 9}$ & 1,97 \\
9 & 2,14 & $\mathbf{1 , 8 7}$ & 1,99 \\
10 & 2,14 & $\mathbf{1 , 9 0}$ & 1,96 \\
\hline
\end{tabular}

procedimento de otimização requer algum conhecimento a priori do problema — dificilmente disponível na prática - e recursos computacionais consideráveis. Conforme esperado, quanto mais recursos computacionais estão disponíveis, i.e., conforme se aumenta $n_{p}$, a diferença entre a performance do OMR-EM e AEs é reduzida, dado que é dado tempo suficiente (ou chances suficientes no caso do OMR-EM) para se alcançar ou chegar perto do ótimo global.

Após tais observações gerais, serão analisados alguns resultados específicos. A Figura 3.9 apresenta alguns resultados representativos para seis bases de dados. Nestes gráficos, o eixo $y$ mostra os valores de MDL (divididos pelo número de objetos), enquanto que o eixo $x$ representa o número de repetições, $n_{p}$, executadas pelo OMR-EM.

Para a base de dados 49Gauss (Figura 3.9(a)), o algoritmo ESM-EM obteve melhores resultados do que ambos OMR-EM e GA-EM. Resultados similares foram observados para a base de dados 9Gauss. Considerando $n_{p} \geq 2$, ESM-EM obteve melhores resultados com menor variância. Como $n_{p}=2$ pode ser considerado um valor muito pequeno na prática, especialmente para uma base de dados com 4.900 objetos, a eficiência computacional do ESM-EM é atraente. Na base de dados Pendigits (Figura 3.9(b)), OMR-EM tem maior variância para $n_{p} \leq 4$ quando comparado com os AEs. A capacidade dos AEs em gerar boas soluções consistentemente em tão pouco tempo é evidenciada. Além disso, novamente o ESM-EM forneceu soluções melhores que o (otimizado) GA-EM. Os resultados para a base de dados c0.5-k12M4 (Figura 3.9(c)) e c1.0-k12M8 (Figura 3.9(d)) apresentam uma tendência similar, mas com o GA-EM apresentando resultados iguais ou ligeiramente melhores que o ESM-EM. Porém, como o ESM-EM não possui parâmetros críticos, ele pode ser mais interessante que o GA-EM até para estes cenários. Para a base de dados $\omega 0.1-k 12 \mathrm{M} 4$, ESM-EM foi capaz de obter boas soluções em uma quantidade pequena de tempo $\left(n_{p}=4\right)$ e resultados competitivos quando mais tempo de processamento estava disponível. Resultados similares foram obtidos para a base de dados $\omega 0.1-\mathrm{k} 12 \mathrm{M} 8$. Os resultados para a base de dados $\omega 0.01-\mathrm{k} 12 \mathrm{M} 8$ são um exemplo de como ineficiente o OMR-EM pode ser quando comparado com busca evolutiva (guiada). Uma tendência similar pode ser observada quando compara-se o ESM-EM e o GA-EM. Especificamente, novamente o ESM-EM convergiu rapidamente, mas neste o caso os outros algoritmos não obtiveram soluções melhores. 


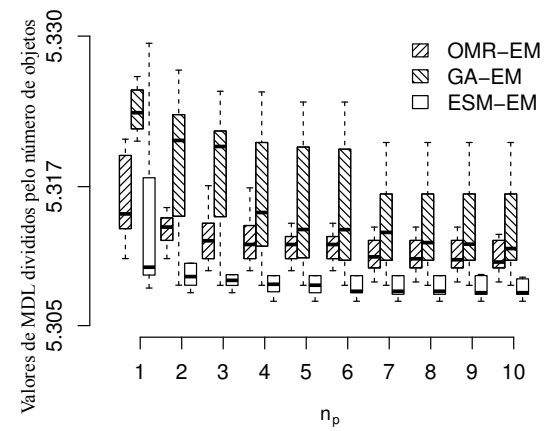

(a) 49Gauss

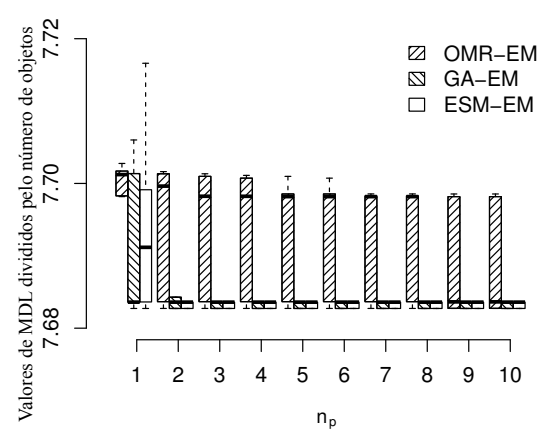

(c) $c 0.5-\mathrm{k} 12 \mathrm{M} 4$

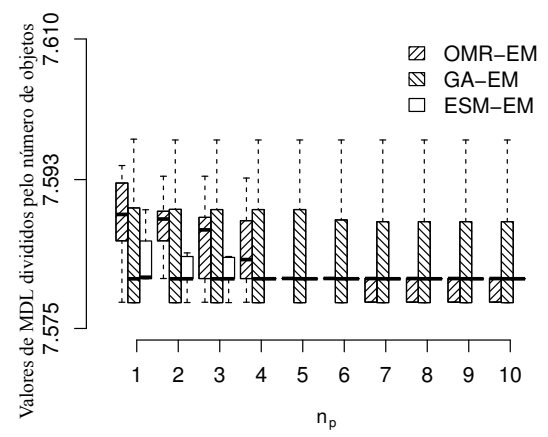

(e) $\omega 0.1-\mathrm{k} 12 \mathrm{M} 4$

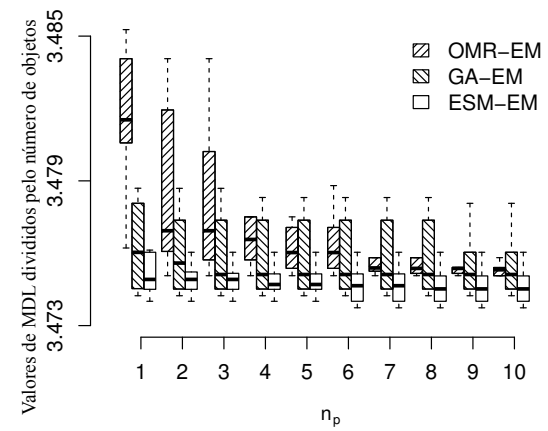

(b) Pendigits

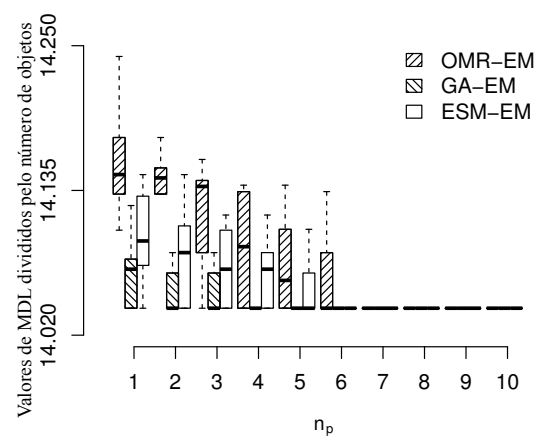

(d) $c 1.0-\mathrm{k} 12 \mathrm{M} 8$

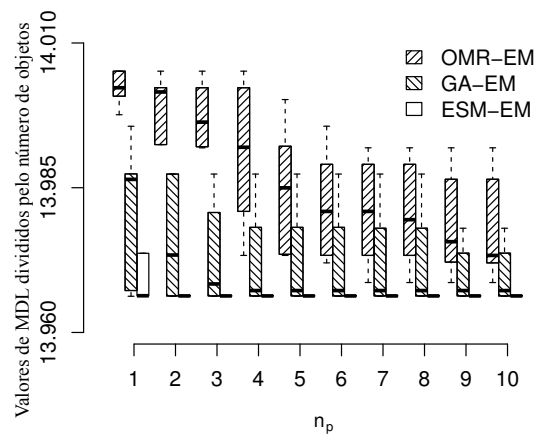

(f) $\omega 0.01-\mathrm{k} 12 \mathrm{M} 8$

Figura 3.9: Boxplot dos valores de MDL (divididos pelo número de objetos) obtidos por cada algoritmo. O eixo $x$ indica o número de repetições $\left(n_{p}\right)$ realizadas pelo OMR-EM.

\subsection{Considerações Finais}

Neste capítulo, foi apresentada uma contribuição desta tese, o algoritmo Evolutionary Split \& Merge Algorithm for Expectation Maximization (ESM-EM) (Covões e Hruschka, 2011). Este algoritmo, desenvolvido com base no Fast Evolutionary Algorithm for Clustering (F-EAC) (Seção 2.4.1.1) e na literatura de SM (Seção 2.3), utiliza os operadores de união e divisão de grupos para auxiliar na evolução dos modelos. Além disso, este algoritmo busca ser uma alternativa aos algoritmos evolutivos existentes nesta linha de pesquisa (descritos na Seção 2.4.2), e foi projetado de tal forma a fazer melhor uso das informações do modelo durante as gerações. Por meio de experimentos com bases de dados sintéticas, foram analisadas diferentes variantes do ESM-EM obtidas alterando alguns de seus componentes. Foi possível demonstrar 
que o ESM-EM é uma alternativa viável ao AE estado-da-arte, denominado de GA-EM (Pernkopf e Bouchaffra, 2005), por ser capaz de prover resultados pelo menos tão bons quanto o GA-EM sem necessitar de otimização de parâmetros e com custo computacional similar. As comparações e análises descritas foram devidamente formalizados em um artigo em revisão em periódico indexado. 


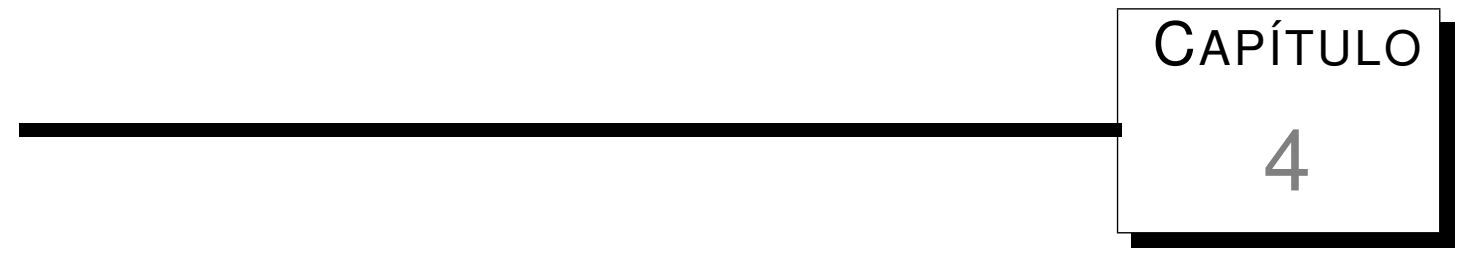

\section{Agrupamento de Dados com Restrições}

\subsection{Considerações Iniciais}

A área de Agrupamento de Dados com Restrições (ADR) — da terminologia em inglês Constrained Clustering — cresceu pela necessidade de se incorporar informações sobre a partição desejada, quando disponíveis, no processo de agrupamento de dados (Basu et al., 2008). O uso de restrições em algoritmos de agrupamento (pela comunidade de Estatísticos) data das décadas de 70 e 80 (Gordon, 1973; Mahajan e Jain, 1978; DeSarbo e Mahajan, 1984). Nas comunidades de aprendizado de máquina e Mineração de Dados (MD) o interesse ocorreu mais recentemente (Wagstaff e Cardie, 2000; Wagstaff et al., 2001) e tem como foco o uso de restrições sobre pares de objetos (Basu et al., 2008). As restrições mais comumente encontradas na literatura são:

- Sobre pares de objetos:

- Must-Link (ML) (Wagstaff e Cardie, 2000): Indica que dois objetos devem ser agrupados no mesmo grupo.

- Cannot-Link (CL) (Wagstaff e Cardie, 2000): Indica que dois objetos devem pertencer a grupos diferentes.

- Sobre grupos:

- Balanced Clusters (Banerjee e Ghosh, 2006): Indica que todos os grupos da partição devem ter aproximadamente a mesma cardinalidade, i.e., o mesmo número de objetos.

- Minimum Cluster Size (Basu et al., 2008): Indica que cada grupo deve ter uma cardinalidade mínima. 
- Sobre partições:

- Non-Redundant Clustering (Gondek e Hofmann, 2004): busca-se partições diferentes (não redundantes) em relação a uma determinada partição de referência.

Neste trabalho, tem-se como premissa o uso das restrições ML e CL por serem mais comuns e, portanto, as mais estudadas na literatura. Diversas aplicações podem ter seu conhecimento de domínio representado por meio de restrições ML e CL. Dentre as mencionadas na literatura, encontram-se: reconhecimento de pista em uma rodovia via GPS (Wagstaff et al., 2001), segmentação de imagens para a navegação de robôs (Davidson e Ravi, 2005b), agrupamento de genes (Zeng et al., 2007) e análise de imagens de ressonância magnética funcional (Phillips et al., 2013).

\subsection{Restrições Must-Link e Cannot-Link e suas Propriedades}

Uma propriedade importante de restrições ML é a transitividade (Wagstaff et al., 2001). Mais especificamente, considere o conjunto de dados $\mathcal{X}=\left\{\mathbf{x}_{n}\right\}_{n=1}^{N}, \mathbf{x}_{n} \in \mathbb{R}^{M}$. Dada uma restrição $\mathrm{ML}$ entre $\mathbf{x}_{i}$ e $\mathbf{x}_{j}(i, j \in\{1, \ldots, N\})$, denotada por $c_{=}(i, j)$, se $c_{=}(i, j)$ e $c_{=}(j, l)$, então $c_{=}(i, l)$ pode ser deduzida. No entanto, o mesmo não é válido para restrições $C L$. Ao se considerar ambos os tipos de restrições, novas restrições CL podem ser deduzidas. Por exemplo, seja uma restrição CL entre $\mathbf{x}_{i}$ e $\mathbf{x}_{j}$, denotada por $c_{\neq}(i, j)$, se $c_{=}(i, j)$ e $c_{\neq}(j, k)$, então $c_{\neq}(i, k)$ pode ser deduzida. Pode-se realizar a dedução de todas as restrições possíveis por meio dos seguintes passos. Inicialmente, considere $\mathcal{M}$ o conjunto de todas as restrições ML fornecidas e $\mathcal{C}$ o conjunto de todas as restrições CL fornecidas. Pode-se então obter um grafo $G=(\mathcal{X}, \mathcal{M})$, i.e., um grafo no qual cada vértice representa um objeto e existe uma aresta entre dois vértices se uma restrição ML entre os dois objetos foi fornecida. Pode-se observar que os componentes conexos ${ }^{1}$ são formados por vértices que representam objetos que devem estar no mesmo grupo. Portanto, dado um componente conexo $C C_{l}$, pode-se inferir $c_{=}(a, b), \forall \mathbf{x}_{a}, \mathbf{x}_{b} \in C C_{l}$. Estes componentes conexos são usualmente denominados chunklets. No caso de restrições $C L$, se existe uma restrição $c_{\neq}(i, j)$, tal que, $\mathbf{x}_{i} \in C C_{l}$ e $\mathbf{x}_{j} \in C C_{m}$, pode-se inferir $c_{\neq}(a, b), \forall \mathbf{x}_{a} \in C C_{l}$ e $\mathbf{x}_{b} \in C C_{m}$ (Basu et al., 2008).

À primeira vista, pode parecer que quanto maior o número de restrições fornecidas melhor seria o desempenho de um dado algoritmo de ADR. No entanto, diversos resultados empíricos indicam que isto não é verdade (Davidson e Ravi, 2006; Davidson et al., 2006; Greene e Cunningham, 2007; Freund et al., 2008; Vu et al., 2010). Existem diversas razões para esta suposição intuitiva não prevalecer na prática. Dentre elas, pode-se destacar: (i) o uso de um número excessivo de restrições pode tornar o problema intratável; (ii) pode haver restrições que

\footnotetext{
${ }^{1}$ Um componente conexo de um grafo $G$ é um subgrafo maximal de $G$ tal que para qualquer par $\{v, w\}$ de seus vértices existe um caminho com extremos $v$ e $w$ (Cormen et al., 2001).
} 
não forneçam informações úteis; (iii) dependendo da forma de aquisição, algumas restrições podem ser errôneas.

Em Davidson et al. (2006), são propostas duas medidas para avaliar a qualidade de restrições: informatividade e coerência. A primeira medida, dependente do algoritmo, contabiliza o número de restrições de um dado conjunto que o algoritmo de ADR satisfaz mesmo sem ser informado sobre elas, i.e., por meio do seu próprio viés (bias) indutivo. A segunda medida analisa a sobreposição da projeção de restrições ML sob CL (e vice-versa), sendo que a existência de sobreposição indica que o par de restrições é incoerente. Por meio destas medidas, é possível filtrar o conjunto, visando reduzir o custo computacional e melhorar as informações fornecidas ao algoritmo de ADR.

Uma alternativa para a obtenção de restrições mais úteis é por meio de seleção ativa de restrição (active constraint selection), na qual são selecionados pares de objetos para os quais é interessante, de acordo com algum critério, que o usuário forneça as restrições (ML ou CL) entre eles. Pode-se citar como exemplos os trabalhos de Basu et al. (2004a), cujo critério é baseado na distância entre objetos, Greene e Cunningham (2007), no qual o critério é baseado em matrizes de co-associação obtidas por ensembles, e Vu et al. (2010), em que o critério adotado é baseado nos vizinhos mais próximos em comum entre os objetos. No entanto, essa abordagem nem sempre é possível devido à indisponibilidade, ou custo excessivo, de um especialista para responder as consultas.

Sob a perspectiva de agrupamento de dados, é importante fazer uma ressalva em relação a restrições ML. Pelo fato das restrições serem obtidas, usualmente, considerando um determinado conceito ao qual o usuário quer diferenciar, estas podem fazer pouco sentido quando consideradas sob o espaço de atributos utilizado. Um exemplo comum dessa situação é quando as restrições são obtidas a partir de rótulos de classes em problemas de classificação. Dado que uma classe pode estar dividida em mais de uma região no espaço de atributos, é possível que a hipótese de um grupo por classe, que é implicitamente feita ao transformar rótulos em restrições, seja inválida. Esta situação é ilustrada na Figura 4.1, na qual a classe 'o' consiste em dois grupos e a classe ' $\mathrm{x}$ ' consiste em três grupos. É fácil notar que não há maneira, neste espaço de atributos, de particionar esses dados em dois grupos de acordo com os rótulos das classes. Portanto, nestes cenários, restrições ML podem ser prejudiciais ao particionamento dos dados, já que dependendo das restrições fornecidas pode-se, entre outras coisas, ocasionar a união de grupos de objetos de classes distintas.

Existem duas abordagens principais para utilizar as restrições: a incorporação delas no processo de agrupamento e o aprendizado de uma medida de distância específica para o problema (Basu et al., 2008). Na primeira, existem duas frentes ativas de pesquisa: algoritmos que buscam partições que satisfazem todas as restrições (Wagstaff e Cardie, 2000; Wagstaff et al., 2001; Klein et al., 2002; Shental et al., 2003; Davidson e Ravi, 2005a; Li et al., 2008; Rothaus e Jiang, 2008; Lu e Peng, 2008; Davidson e Ravi, 2009), e algoritmos que buscam minimizar o número de violações de restrições (Basu et al., 2004b; Bilenko et al., 2004; Basu et al., 2004a; Lange 


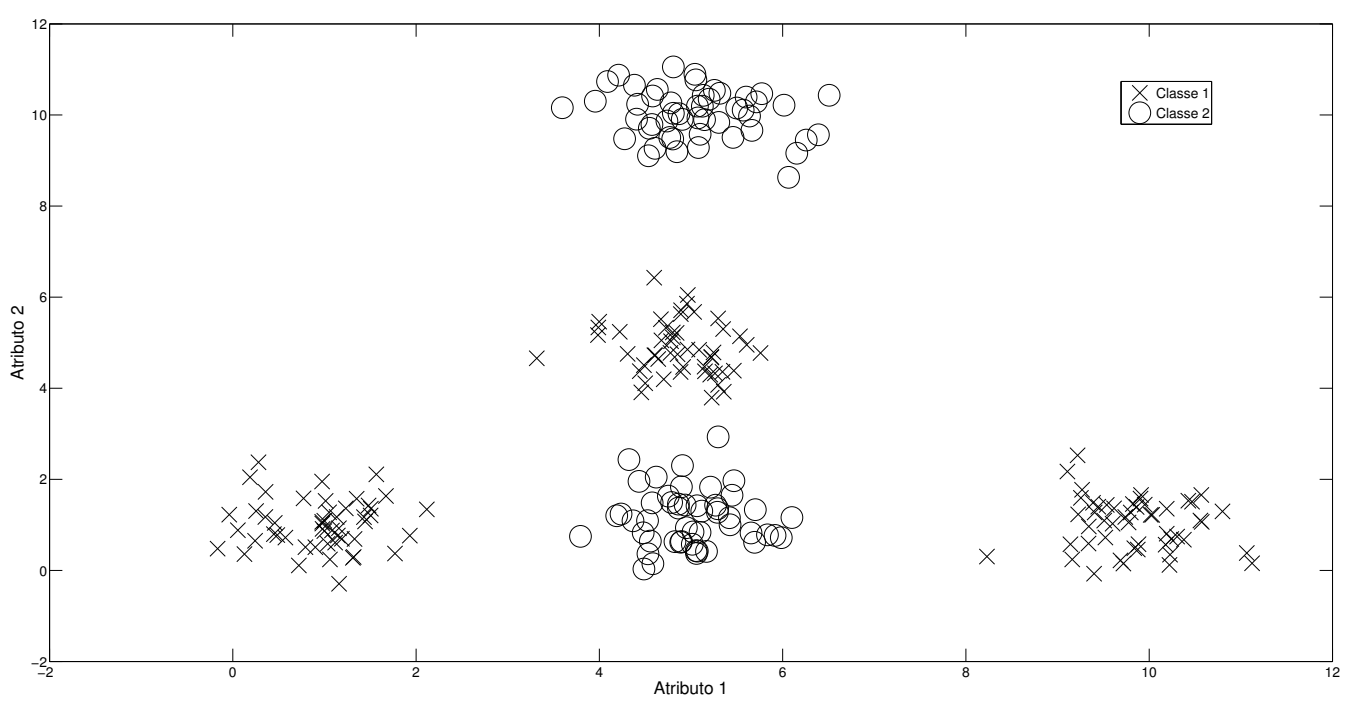

Figura 4.1: Exemplo de distinção entre grupos e classes.

et al., 2005; Zhao e Miller, 2005; Davidson e Ravi, 2005b; Law et al., 2005; Grira et al., 2006; Pelleg e Baras, 2007; Lu e Leen, 2007a,b; Hu et al., 2008a,b; Ares et al., 2009; Costa et al., 2009; Benabdeslem e Snoussi, 2009; Li et al., 2009; Zhang e Wong, 2009; Wang et al., 2014; Raghuram et al., 2014). Na literatura de minimização do número de violações de restrições, as restrições são usualmente chamadas de soft constraints, e alguns trabalhos adotam uma generalização no conceito de restrições na qual, para cada restrição (ML ou CL), é informada também a confiança do usuário naquela restrição. Conforme já mencionado, neste trabalho tem-se como foco algoritmos que atuam sobre partições rígidas ou probabilísticas dos dados. Por tal razão, algoritmos de ADR baseados em partições fuzzy não estão relacionados. Sugere-se ao leitor interessado em algoritmos deste gênero os trabalhos de Bouchachia e Pedrycz (2006) e Medeiros (2010).

Na abordagem baseada no aprendizado de medida de distância, uma restrição ML entre os objetos $\mathbf{x}_{i}$ e $\mathbf{x}_{j}$ pode ser interpretada como uma indicação de que a distância entre os dois objetos é pequena. Analogamente, uma restrição CL entre os objetos indicaria que a distância entre eles é grande e, portanto, os mesmos não deveriam pertencer ao mesmo grupo (Wagstaff et al., 2001). Desta forma, é induzida uma métrica de distância $\left\|\mathbf{x}_{i}-\mathbf{x}_{j}\right\|_{\mathbf{A}}, \mathbf{A} \in \mathbb{R}^{M \times M}$ em que $M$ é o número de atributos da base de dados (Xing et al., 2002; Bilenko et al., 2004; Basu et al., 2004b; Bar-Hillel et al., 2005; Chatel et al., 2014). Tais trabalhos podem ser convenientemente categorizados em: algoritmos que aprendem uma métrica por grupo (Bilenko et al., 2004) e aqueles que aprendem uma métrica para todos os dados (Xing et al., 2002; Basu et al., 2004b; Bar-Hillel et al., 2005; Chatel et al., 2014); bem como entre algoritmos que realizam apenas o aprendizado de métrica (Xing et al., 2002; Bar-Hillel et al., 2005; Chatel et al., 2014) e os que integram o aprendizado de métrica no processo de agrupamento (Basu et al., 2004b; Bilenko et al., 2004). 


\subsection{Algoritmos que Utilizam Restrições Must-Link e Cannot- Link}

Neste trabalho, tem-se interesse nos algoritmos de agrupamento que incorporam as restrições no processo de particionamento. Conforme mencionando anteriormente, alguns algoritmos de ADR não permitem a violação de restrições no processo de agrupamento-i.e., em todas as iterações do algoritmo as partições resultantes devem satisfazer todas as restrições. Embora isto possa ser interessante em algumas circunstâncias, as restrições são usualmente fornecidas por usuários que desconhecem a disposição dos dados no espaço. Dessa forma, a necessidade de satisfazer todas as restrições pode tornar o processo de agrupamento intratável, e uma partição vazia é comumente retornada neste caso. Para ilustrar isso, considere a Figura 4.2, que apresenta as áreas de pétalas e de sépalas para a amplamente conhecida base de dados Iris $^{2}$. As três classes são representadas por diferentes marcadores e o centróide ${ }^{3}$ de cada classe é representado por um ponto. Os marcadores envoltos por retângulos correspondem a alguns objetos para os quais o centróide mais próximo não é o centróide de sua respectiva classe. Diferentemente do exemplo ilustrado na Figura 4.1, neste caso a hipótese de que cada classe corresponde a um grupo distinto é válida ${ }^{4}$. No entanto, um algoritmo de ADR baseado no algoritmo $K$-means não será capaz de satisfazer algumas restrições derivadas a partir das classes sem obter centróides diferentes dos originais. Por tal razão, algoritmos que permitem a violação de restrições - e.g., baseados em soft constraints - tendem a ser mais interessantes em alguns casos, por terem como objetivo encontrar uma partição que esteja de acordo com as restrições (i.e., expectativas a priori) do usuário tanto quanto possível. Note que um algoritmo que não seja baseado na hipótese de um único grupo por classe pode ser capaz de lidar com esse tipo de problema, mesmo respeitando todas as restrições. Isso ocorre devido à possibilidade de criar subgrupos disjuntos, aliviando o problema de sobreposição entre grupos. Esta perspectiva será discutida em mais detalhes nos Capítulos 6 e 7.

Diversos algoritmos de ADR se concentram na adaptação do algoritmo $K$-means (Steinley, 2006; Jain, 2010), e.g., Wagstaff et al. (2001); Bilenko et al. (2004); Davidson e Ravi (2005b); Pelleg e Baras (2007); Rothaus e Jiang (2008); Hu et al. (2008a); Hong et al. (2008); Zhang e Wong (2009); Ares et al. (2009). É importante ressaltar que o algoritmo K-means pode ser visto como uma versão específica do algoritmo Expectation Maximization (EM) para Gaussian Mixture Models (GMMs) (McLachlan e Peel, 2000), na qual se supõe que grupos são gerados por distribuições gaussianas com matriz de covariância diagonais e proporcionais a matriz identidade e cada objeto é oriundo de apenas uma distribuição (Bishop, 2006). Alguns trabalhos buscam adaptar este modelo, mais genérico, para incorporar as restrições, e.g., Shental et al. (2003); Lange et al. (2005); Law et al. (2005); Zhao e Miller (2005); Lu e Leen (2007a);

\footnotetext{
${ }^{2}$ Para melhor visualização, alguns objetos distantes de áreas densas foram omitidos.

${ }^{3}$ Vetor médio de um grupo de objetos (Xu e Wunsch, 2009).

${ }^{4}$ Considerando a hipótese de que grupos podem ter (algum nível de) sobreposição.
} 


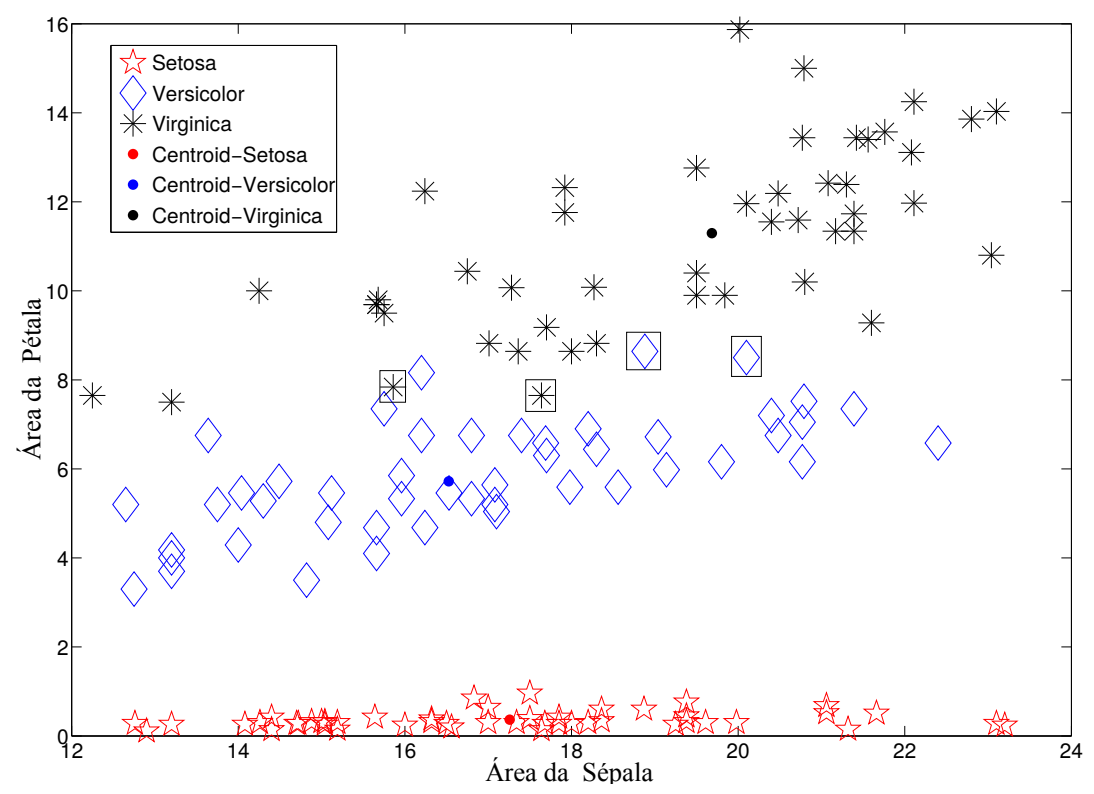

Figura 4.2: Base de dados Iris — retângulos destacam alguns objetos para os quais o centróide mais próximo (de acordo com a distância euclidiana) não é o centróide de sua respectiva classe.

Raghuram et al. (2014). Tanto o $K$-means quanto o EM estão entre os 10 algoritmos mais influentes em MD (Wu et al., 2008). Por tal razão, neste trabalho têm-se como foco o estudo de soluções para o problema de ADR baseados nestes algoritmos.

\subsubsection{Algoritmos Baseados no K-means}

O K-means (Steinley, 2006; Jain, 2010) é provavelmente o algoritmo baseado no critério do erro quadrático mais simples e mais utilizado na prática. Este critério é o mais intuitivo e frequentemente empregado em algoritmos de agrupamento particionais (Jain et al., 1999). Uma das principais razões para a ampla adoção do algoritmo $K$-means na prática é sua eficiência computacional, dado que o custo computacional do algoritmo é $O(t \cdot K \cdot M \cdot N)$, sendo $t$ o número de iterações, $K$ o número de grupos, $M$ o número de atributos e, finalmente, $N$ o número de objetos. A função objetivo do $K$-means consiste na minimização do erro quadrático, definido como:

$$
J_{K-\text { means }}=\sum_{k=1}^{K} \sum_{\mathbf{x}_{n} \in C_{k}}\left\|\mathbf{x}_{n}-\mathbf{c}_{k}\right\|^{2},
$$

sendo $C_{k}$ o conjunto de objetos pertencentes ao grupo $k, \mathbf{c}_{k}=\left|C_{k}\right|^{-1} \sum_{\mathbf{x}_{n} \in C_{k}} \mathbf{x}_{n}$ o centróide do grupo $k$ e $\left\|\mathbf{x}_{n}-\mathbf{c}_{k}\right\|^{2}=\left(\mathbf{x}_{n}-\mathbf{c}_{k}\right)^{T}\left(\mathbf{x}_{n}-\mathbf{c}_{k}\right)$ a distância euclidiana entre um objeto e um centróide.

O K-means é apresentado no Algoritmo 11. Diversos critérios de convergência podem ser utilizados, dentre os mais comuns estão um número máximo de iterações ou uma diferença mínima entre centróides de iterações consecutivas. Conforme pode ser visto pelo Algoritmo 11, o K-means é sensível aos centróides iniciais fornecidos ao algoritmo. Para suprir esta limitação, 
diversas abordagens têm sido investigadas na literatura (Arthur e Vassilvitskii, 2007; Bahmani et al., 2012). Outro fator limitante do algoritmo é a necessidade de informar o número de grupos desejado, apesar de o mesmo ser frequentemente desconhecido na prática de MD (Xu e Wunsch, 2009). Apesar disso, o K-means é utilizado em diversas aplicações, dentre as quais pode-se mencionar: segmentação de imagens de microscópio (Dima et al., 2011), monitoração de tremores vulcânicos (Messina e Langer, 2011) e alocação eficiente de tarefas a múltiplos robôs (Elango et al., 2011).

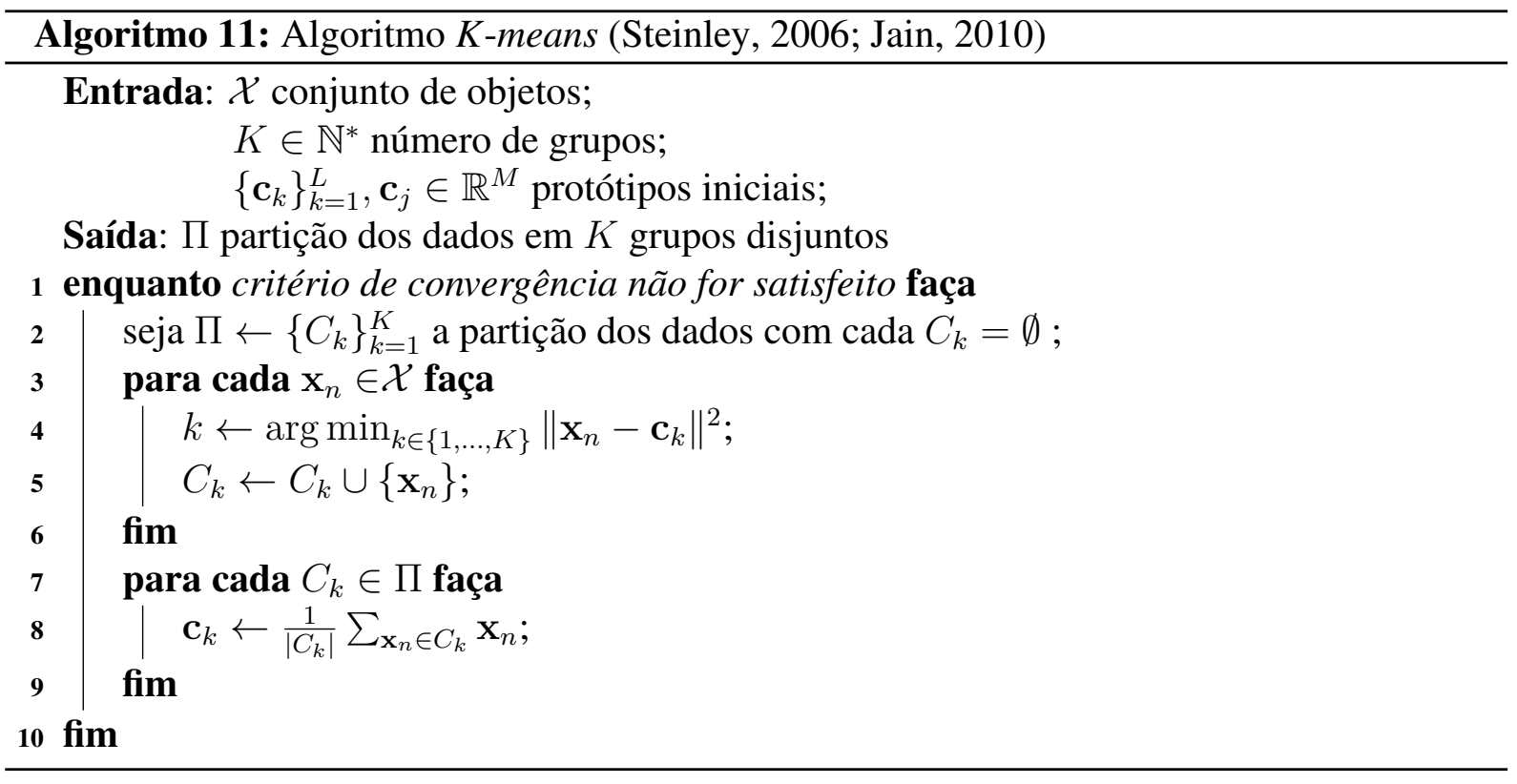

Por todas as razões supramencionadas, o algoritmo $K$-means é utilizado como base para o desenvolvimento de diversos algoritmos na literatura. No contexto de ADR, o algoritmo COP-KMEANS proposto por Wagstaff et al. (2001) foi o pioneiro na incorporação do uso de restrições no $K$-means. Neste algoritmo, os objetos são rotulados para o grupo com centróide mais próximo e que não viola nenhuma restrição. Caso não seja possível atribuir um objeto a um grupo sem violar alguma restrição, uma partição vazia é retornada. Rothaus e Jiang (2008) propuseram uma versão modificada do $K$-means que considera uma modificação na forma com que a distância entre objetos é calculada, além de utilizarem o conceito de subgrupos. No trabalho de Hu et al. (2008a), uma representação alternativa do $K$-means é utilizada baseada na maximização do traço de uma matriz para a computação do erro quadrático. A incorporação das restrições é dada por meio de um fator de penalização para soluções que violam restrições. No entanto, este fator conta com um parâmetro crítico que determina o grau de importância de satisfazer restrições. Zhang e Wong (2009) utilizam tanto active constraint selection quanto múltiplos subgrupos por grupos para buscar por partições que violem o mínimo de restrições possível. Porém, o usuário deve definir quantos subgrupos cada grupo deve possuir. O algoritmo proposto em Ares et al. (2009) é baseado em uma divisão nas restrições fornecidas, i.e., o usuário fornece restrições que devem ser obrigatoriamente atendidas e restrições que podem ser violadas. As restrições que devem ser atendidas são tratadas de forma igual ao COP-KMEANS 
(Wagstaff et al., 2001). No entanto, para buscar atender as restrições que podem ser violadas, é utilizado um peso que aumenta (ou diminui) a distância entre um objeto e determinado grupo. Hong et al. (2008) propuseram um algoritmo genético para evoluir partições que minimizem o número de restrições violadas. Devido a este algoritmo ser de maior interesse para este trabalho, o mesmo é descrito em maiores detalhes na Seção 4.3.1.4.

Nas próximas seções, são descritos os algoritmos propostos por Davidson e Ravi (2005b), Pelleg e Baras (2007) e Bilenko et al. (2004), respectivamente. Estes algoritmos foram analisados por meio de experimentos empíricos descritos em Covões et al. (2013c). É importante notar que todos os algoritmos citados partem de três premissas: (i) o usuário define o número de grupos na base de dados; (ii) as restrições assumem um grupo por classe; (iii) todo o conjunto de dados $\mathcal{X}$ está disponível a priori para o processamento. No Capítulo 5 são propostos dois algoritmos para o agrupamento online dos dados e que consideram restrições. No agrupamento online a partição é atualizada a cada objeto ou subconjunto de objetos processado. Esse tipo de algoritmo é especialmente útil em problemas de agrupamento de dados com muitos objetos, nos quais não se deseja carregar todos os dados na memória ou processar várias vezes o mesmo conjunto de objetos. Portanto, tais algoritmos não partem da premissa (iii). Conforme mencionado anteriormente, nos Capítulos 6 e 7 são apresentados dois algoritmos que não assumem as premissas (i) e (ii).

Para detalhar tais algoritmos, é necessário definir algumas notações. Assume-se que cada algoritmo recebe um conjunto $\mathcal{M}$ de restrições $\mathrm{ML}$ e um conjunto $\mathcal{C}$ de restrições CL. Sendo $o_{\mathcal{M}}(l)$ e $o_{\mathcal{M}}^{\prime}(l)$, funções que retornam o primeiro e o segundo objeto da $l$-ésima restrição ML, pode-se definir as funções $g_{\mathcal{M}}(l)$ e $g_{\mathcal{M}}^{\prime}(l)$ que retornam, respectivamente, o índice do grupo ao qual o primeiro e o segundo objeto da $l$-ésima restrição ML pertencem, i.e., $g_{\mathcal{M}}(l)=$ $\left\{j \mid o_{\mathcal{M}}(l) \in C_{j}\right\}$ e $g_{\mathcal{M}}^{\prime}(l)=\left\{t \mid o_{\mathcal{M}}^{\prime}(l) \in C_{t}\right\}$. De forma análoga, as funções $o_{\mathcal{C}}(l), o_{\mathcal{C}}^{\prime}(l), g_{\mathcal{C}}(l)$ e $g_{\mathcal{C}}^{\prime}(l)$ podem ser definidas para as restrições CL. O conjunto de restrições ML sendo violadas é definido como $\mathcal{V}_{\mathcal{M}}=\left\{i \mid \mathcal{M}_{i} \in \mathcal{M}, g_{\mathcal{M}}(i) \neq g_{\mathcal{M}}^{\prime}(i)\right\}$ e, semelhantemente, o conjunto de restrições $\mathrm{CL}$ sendo violadas é definido como $\mathcal{V}_{\mathcal{C}}=\left\{i \mid \mathcal{C}_{i} \in \mathcal{C}, g_{\mathcal{C}}(i)=g_{\mathcal{C}}^{\prime}(i)\right\}$. Definidas estas notações, na sequência são apresentados os algoritmos analisados.

\subsubsection{Constrained Vector Quantization Error}

O algoritmo Constrained Vector Quantization Error (CVQE), proposto por Davidson e Ravi (2005b), emprega a função objetivo do algoritmo $K$-means incrementada de dois termos que consideram os custos de violação de restrições. Informalmente, o custo da violação de uma restrição ML é a distância entre os protótipos dos dois grupos que contêm os objetos que deveriam estar no mesmo grupo. No caso da violação de uma restrição CL, o custo é definido como a distância entre o protótipo do grupo em que os dois objetos estão contidos e o protótipo vizinho mais próximo. Formalmente, a função objetivo é definida como: 


$$
\begin{gathered}
J_{C V Q E}=\sum_{k=1}^{K} J_{C V Q E_{k}}, \\
J_{C V Q E_{k}}=\frac{1}{2} \sum_{\mathbf{x}_{n} \in C_{k}}\left\|\mathbf{c}_{k}-\mathbf{x}_{n}\right\|^{2}+\frac{1}{2} \sum_{l \in \mathcal{V}_{\mathcal{M}, g_{\mathcal{M}}(l)=k}}\left\|\mathbf{c}_{k}-\mathbf{c}_{g_{\mathcal{M}}^{\prime}(l)}\right\|^{2}+\frac{1}{2} \sum_{l \in \mathcal{V}_{\mathcal{C}}, g_{\mathcal{C}}(l)=k}\left\|\mathbf{c}_{k}-\mathbf{c}_{h\left(g_{\mathcal{C}}^{\prime}(l)\right)}\right\|^{2},
\end{gathered}
$$

em que $h(i)$ retorna o índice do grupo cujo protótipo é o mais próximo do $i$-ésimo grupo.

O algoritmo CVQE atribui os objetos aos grupos da seguinte forma: (i) objetos que não estão envolvidos em nenhuma restrição são atribuídos ao grupo com o protótipo mais próximo; (ii) pares de objetos envolvidos em restrições, tanto ML quanto CL, são atribuídos aos grupos que minimizam a função objetivo - Equação (4.2). Para tal, todas as combinações são verificadas (Davidson e Ravi, 2005b). Os protótipos $\left(\mathbf{c}_{k}, k=\{1, \ldots, K\}\right)$ são então atualizados de acordo com:

$$
\mathbf{c}_{k}=\frac{\sum_{\mathbf{x}_{n} \in C_{k}} \mathbf{x}_{n}+\sum_{l \in \mathcal{V}_{\mathcal{M}}, g_{\mathcal{M}}(l)=k} \mathbf{c}_{g_{\mathcal{M}}^{\prime}(l)}+\sum_{l \in \mathcal{V}_{\mathcal{C}}, g_{\mathcal{C}}(l)=k} \mathbf{c}_{h\left(g_{\mathcal{C}}^{\prime}(l)\right)}}{\left|C_{k}\right|+\sum_{l \in \mathcal{V}_{\mathcal{M}}} \mathbb{1}_{\left[g_{\mathcal{M}}(l)=k\right]}+\sum_{l \in \mathcal{V}_{\mathcal{C}}} \mathbb{1}_{\left[g_{\mathcal{C}}(l)=k\right]}} .
$$

Pode-se interpretar a atualização do protótipo da seguinte maneira (Davidson e Ravi, 2005b): se uma restrição ML é violada, o protótipo do grupo que contém o primeiro objeto da restrição é movido na direção do grupo contendo o segundo objeto da restrição. No caso de violação de uma restrição CL, o protótipo do grupo que contém os dois objetos da restrição é movido na direção do grupo vizinho mais próximo do segundo objeto da restrição.

O custo computacional de uma iteração do algoritmo CVQE é $O(K \cdot M \cdot(N+|\mathcal{M}|+$ $\left.|\mathcal{C}|)+K^{2} \cdot(M+|\mathcal{M}|+|\mathcal{C}|)\right)$. Conforme pode ser visto, este algoritmo mantém algumas propriedades interessantes do $K$-means, como o custo linear em relação aos objetos e atributos. No entanto, é introduzido um custo quadrático em relação ao número de grupos, o que o torna computacionalmente custoso em aplicações em que o número de grupos é alto.

Conforme discutido em Pelleg e Baras (2007), o algoritmo CVQE possui algumas limitações. Por exemplo, o algoritmo é sensível à ordem dos objetos em cada restrição. Isto é facilmente observável pelo cálculo da atualização do protótipo no caso da violação de uma restrição ML, no qual apenas o protótipo referente ao grupo do primeiro objeto da restrição é afetado. Além disso, para verificar todas as possíveis combinações de grupos são necessários $O\left(K^{2}\right)$ cálculos. Finalmente, no caso de violação de restrições, apenas as distâncias entre protótipos são consideradas na penalização, i.e., a posição dos objetos em relação a estes protótipos é ignorada. Buscando contornar estas limitações, uma variante deste algoritmo, denominada Linear Constrained Vector Quantization Error, foi apresentada por Pelleg e Baras (2007). 


\subsubsection{Linear Constrained Vector Quantization Error}

O algoritmo Linear Constrained Vector Quantization Error (LCVQE) utiliza uma versão modificada da função objetivo do CVQE - Equação (4.2). Informalmente, o custo de violar uma restrição ML passa a ser a média da distância entre cada objeto da restrição e o protótipo do grupo ao qual o outro objeto pertence. Para uma restrição CL, o objeto da restrição mais distante do protótipo do seu grupo é inicialmente definido e, então, a distância entre esse objeto e o protótipo vizinho mais próximo é usada como custo da violação (Pelleg e Baras, 2007). Portanto, a função objetivo $J_{L C V Q E}$ é definida por:

$$
\begin{gathered}
J_{L C V Q E}=\sum_{k=1}^{K} J_{L C V Q E_{k}} \\
J_{L C V Q E_{k}}=\frac{1}{2} \sum_{\mathbf{x}_{n} \in C_{k}}\left\|\mathbf{c}_{k}-\mathbf{x}_{n}\right\|^{2}+\frac{1}{2} \sum_{l \in \mathcal{V}_{\mathcal{M}}, g_{\mathcal{M}}(l)=k} \frac{1}{2}\left\|\mathbf{c}_{k}-o_{\mathcal{M}}^{\prime}(l)\right\|^{2} \\
+\frac{1}{2} \sum_{l \in \mathcal{V}_{\mathcal{M}}, g_{\mathcal{M}}^{\prime}(l)=k} \frac{1}{2}\left\|\mathbf{c}_{k}-o_{\mathcal{M}}(l)\right\|^{2}+\frac{1}{2} \sum_{l \in \mathcal{V}_{\mathcal{C}}, V(l)=k}\left\|\mathbf{c}_{k}-R_{g_{\mathcal{C}}(l)}(l)\right\|^{2}
\end{gathered}
$$

As funções auxiliares, $R_{k}(l)$ e $V(l)$, são definidas nas Equações (4.7) e (4.8), respectivamente. Intuitivamente, a primeira $-R_{k}(l)-$ retorna o objeto da $l$-ésima restrição CL mais distante do protótipo $\mathbf{c}_{k}$, enquanto a segunda $-V(l)$ - retorna o índice do protótipo vizinho mais próximo ao objeto $R_{g_{\mathcal{C}}(l)}(l)$, que é o objeto da $l$-ésima restrição CL mais distante do protótipo do seu grupo, isto é:

$$
\begin{gathered}
R_{k}(l)= \begin{cases}o_{\mathcal{C}}(l) & \text { se }\left\|o_{\mathcal{C}}(l)-\mathbf{c}_{k}\right\|^{2}>\left\|o_{\mathcal{C}}^{\prime}(l)-\mathbf{c}_{k}\right\|^{2} \\
o_{\mathcal{C}}^{\prime}(l) & \text { caso contrário, }\end{cases} \\
V(l)=\underset{m \in\{1, \ldots, K\} \backslash\left\{g_{\mathcal{C}}(l)\right\}}{\arg \min }\left\|R_{g_{\mathcal{C}}(l)}(l)-\mathbf{c}_{m}\right\|^{2} .
\end{gathered}
$$

A atribuição dos objetos envolvidos em restrições aos grupos também difere do CVQE. Inicialmente, todos os objetos são atribuídos ao grupo cujo protótipo está mais próximo. Para cada restrição ML sendo violada, apenas três possibilidades de atribuição são examinadas: (i) ignorar a violação, mantendo a atribuição de cada objeto ao grupo de protótipo mais próximo; (ii) atribuir os dois objetos ao grupo de protótipo mais próximo do primeiro objeto $\left(o_{\mathcal{M}}(l)\right)$; (iii) atribuir os dois objetos ao grupo de protótipo mais próximo do segundo objeto $\left(o_{\mathcal{M}}^{\prime}(l)\right)$. Para cada restrição CL sendo violada, apenas dois casos são verificados: (i) manter a violação, mantendo a atribuição dos dois objetos ao grupo de protótipo mais próximo; (ii) manter o objeto mais próximo do protótipo nesse grupo, e atribuir o objeto mais distante $\left(R_{g_{\mathcal{C}}(l)}(l)\right)$ ao grupo do protótipo vizinho mais próximo $(V(l))$. Feitas as devidas considerações sobre alocações de objetos aos grupos, a atualização dos protótipos é dada por: 


$$
\mathbf{c}_{k}=\frac{\sum_{\mathbf{x}_{n} \in C_{k}} \mathbf{x}_{n}+\frac{1}{2} \sum_{l \in \mathcal{V}_{\mathcal{M}}, g_{\mathcal{M}}(l)=k} o_{\mathcal{M}}^{\prime}(l)+\frac{1}{2} \sum_{l \in \mathcal{V}_{\mathcal{M}}, g_{\mathcal{M}}^{\prime}(l)=k} o_{\mathcal{M}}(l)+\sum_{l \in \mathcal{V}_{\mathcal{C}}, V(l)=k} R_{g_{\mathcal{C}}(l)}(l)}{\left|C_{k}\right|+\frac{1}{2} \sum_{l \in \mathcal{V}_{\mathcal{M}}} \mathbb{1}_{\left[g_{\mathcal{M}}(l)=k\right]}+\frac{1}{2} \sum_{l \in \mathcal{V}_{\mathcal{M}}} \mathbb{1}_{\left[g_{\mathcal{M}}^{\prime}(l)=k\right]}+\sum_{l \in \mathcal{V}_{\mathcal{C}}} \mathbb{1}_{[V(l)=k]}} .
$$

É possível interpretar a atualização do protótipo da seguinte forma. Seja $l$ uma restrição ML que está sendo violada, i.e., $o_{\mathcal{M}}(l) \in C_{k}$ e $o_{\mathcal{M}}^{\prime}(l) \in C_{k_{1}}$ com $k \neq k_{1}$. Então, o protótipo $\mathbf{c}_{k}$ é movido em direção ao objeto $o_{\mathcal{M}}^{\prime}(l)$ e o protótipo $\mathbf{c}_{k_{1}}$ é movido em direção ao objeto $o_{\mathcal{M}}(l)$. Considere agora o caso de uma restrição CL estar sendo violada, ou seja, $o_{\mathcal{C}}(l) \in C_{k}$ e $o_{\mathcal{C}}^{\prime}(l) \in$ $C_{k}$. Considere também que $\left\|\mathbf{c}_{k}-o_{\mathcal{C}}^{\prime}(l)\right\|^{2}>\left\|\mathbf{c}_{k}-o_{\mathcal{C}}(l)\right\|^{2}$, e que $\mathbf{c}_{k_{1}}$ é o protótipo vizinho mais próximo de $o_{\mathcal{C}}^{\prime}(l)$. Então, o protótipo $\mathbf{c}_{k_{1}}$ é movido na direção de $o_{\mathcal{C}}^{\prime}(l)$. Considerando estas operações, o custo computacional de uma iteração do LCVQE é $O(K \cdot M \cdot(N+|\mathcal{M}|+$ $|\mathcal{C}|)$ ). Portanto, o algoritmo incorpora as restrições no processo de agrupamento de forma mais eficiente que o algoritmo CVQE. Isto se deve ao uso da heurística para reduzir o número de possíveis atribuições para cada objeto envolvido em uma restrição violada, e por considerar apenas distâncias entre objetos e centróides, tornando desnecessário o cálculo da distância entre todos os pares de centróides.

\subsubsection{Metric Pairwise Constrained K-Means}

O algoritmo Metric Pairwise Constrained K-Means (MPCK-Means) (Bilenko et al., 2004) busca aprender uma métrica de distância que melhor se adapte às restrições. Especificamente, para cada grupo $C_{k}$ é otimizada uma matriz semi-definida positiva ${ }^{5}, \mathbf{A}_{k}$, que parametriza a distância euclidiana da seguinte forma: $\left\|\mathbf{x}_{n}-\mathbf{c}_{k}\right\|_{\mathbf{A}_{k}}=\sqrt{\left(\mathbf{x}_{n}-\mathbf{c}_{k}\right)^{T} \mathbf{A}_{k}\left(\mathbf{x}_{n}-\mathbf{c}_{k}\right)}$. Existem outros algoritmos para o aprendizado de métricas de distância utilizando restrições (Xing et al., 2002; Bar-Hillel et al., 2005). Porém, estes não realizam o agrupamento dos dados e permitem o aprendizado de apenas uma mesma métrica para todos os grupos, o que limita os resultados a grupos com formas iguais (Bilenko et al., 2004). A função objetivo do algoritmo MPCK-Means é definida como:

$$
\begin{gathered}
J_{m p c k}=\sum_{k=1}^{K} \sum_{\mathbf{x}_{n} \in C_{k}}\left[\left\|\mathbf{x}_{n}-\mathbf{c}_{k}\right\|_{\mathbf{A}_{k}}^{2}-\log \left(\left|\mathbf{A}_{k}\right|\right)\right]+\sum_{l \in \mathcal{V}_{\mathcal{M}}} w_{l} f_{M L}(l)+\sum_{l \in \mathcal{V}_{\mathcal{C}}} \bar{w}_{l} f_{C L}(l), \\
f_{M L}(l)=\frac{1}{2}\left\|o_{\mathcal{M}}(l)-o_{\mathcal{M}}^{\prime}(l)\right\|_{A_{g_{\mathcal{M}}(l)}}^{2}+\frac{1}{2}\left\|o_{\mathcal{M}}(l)-o_{\mathcal{M}}^{\prime}(l)\right\|_{A_{g_{\mathcal{M}}^{\prime}}(l)}^{2}, \\
f_{C L}(l)=\left\|\mathbf{x}_{d}^{\left(g_{\mathcal{C}}(l)\right)}-\mathbf{x}_{d^{\prime}}^{\left(g_{\mathcal{C}}(l)\right)}\right\|_{A_{g_{\mathcal{C}}(l)}}^{2}-\left\|o_{\mathcal{C}}(l)-o_{\mathcal{C}}^{\prime}(l)\right\|_{A_{g_{\mathcal{C}}(l)}}^{2},
\end{gathered}
$$

${ }^{5}$ Uma matriz A é semi-definida positiva se $\mathbf{v}^{T} \mathbf{A v} \geq 0, \forall \mathbf{v}$ (Golub e Van Loan, 1996). 
em que $-\log \left(\left|\mathbf{A}_{k}\right|\right)$ aparece devido à constante normalizadora do modelo generalizado do $K$ means $^{6}$, e $w_{l}$ e $\bar{w}_{l}$ são pesos definidos pelo usuário para penalizar a violação da l-ésima restrição ML e a $l$-ésima restrição CL, respectivamente. $\left\|\mathbf{x}_{d}^{\left(g_{\mathcal{C}}(l)\right)}-\mathbf{x}_{d^{\prime}}^{\left(g_{\mathcal{C}}(l)\right)}\right\|_{A_{g_{\mathcal{C}}(l)}}^{2}$ representa a maior distância entre dois objetos - considerando a métrica do grupo $C_{g_{\mathcal{C}}(l)}$. As funções auxiliares $f_{M L}$ e $f_{C L}$ calculam a penalização por violar a $l$-ésima restrição ML e CL, respectivamente. A primeira, $f_{M L}$, penaliza a violação de uma restrição ML proporcionalmente à distância entre os objetos. Como uma violação desse tipo envolve dois grupos, o cálculo da distância entre os dois objetos considera as duas métricas de distância. A segunda, $f_{C L}$, penaliza a violação de uma restrição CL de forma que seja inversamente proporcional à distância entre os dois objetos.

O algoritmo MPCK-Means utiliza uma heurística para a inicialização dos protótipos, considerando as restrições fornecidas. Inicialmente, são deduzidas as restrições ML e CL possíveis do conjunto original de restrições. Para simplificar a notação, o conjunto com as restrições ML fornecidas e deduzidas é denotado por $\mathcal{M}$, e de forma similar, forma-se o conjunto $\mathcal{C}$ para as restrições CL. Os chunklets obtidos formam o conjunto de vizinhanças $\Lambda$. Se $|\Lambda| \leq K$, são inicializados $|\Lambda|$ protótipos iniciais como os centróides dos objetos que formam cada vizinhança, $\lambda_{k}$, e os $K-|\Lambda|$ protótipos restantes são inicializados como a média global dos dados adicionada a uma perturbação aleatória. Se $|\Lambda|>K, K$ vizinhanças são escolhidas usando uma variante ponderada do algoritmo farthest-first (Hochbaum e Shmoys, 1985), cujos pesos correspondem ao número de objetos presentes em cada vizinhança. Portanto, este procedimento de inicialização tem um viés em relação a vizinhanças distantes que representam uma grande quantidade de objetos (Bilenko et al., 2004).

Para a atribuição dos objetos aos grupos, o algoritmo MPCK-Means utiliza uma estratégia sensível à ordem de apresentação dos dados. Objetos são atribuídos ao grupo que minimiza o custo de acordo com a Equação (4.10). Considere que o objeto $\mathbf{x}_{n}$ foi atribuído a um determinado grupo. Esta atribuição não é revista na iteração atual, i.e., ela não pode ser modificada, e é apenas considerada para a verificação de possíveis violações de restrições envolvendo outros objetos, assim como para calcular o impacto de tais atribuições na função objetivo. Bilenko et al. (2004) sugerem processar os objetos em uma ordem aleatória a cada iteração.

A atualização dos protótipos é a mesma utilizada pelo $K$-means original, e cada matriz $\mathbf{A}_{k}$ é atualizada de acordo com a Equação (4.13), para a qual alguns cuidados devem ser tomados (Bilenko et al., 2004). Inicialmente, é necessário verificar se o somatório de matrizes de covariância, do lado direito na Equação (4.13), é singular. Caso seja, é adicionada uma fração do traço da matriz na diagonal principal, i.e., $\mathbf{A}_{k}^{-1}=\mathbf{A}_{k}^{-1}+\epsilon \operatorname{trace}\left(\mathbf{A}_{k}^{-1}\right) \mathbf{I}$. Caso a matriz $\mathbf{A}_{k}$ resultante da inversão não seja semi-definida positiva, é necessário projetá-la no conjunto de matrizes semi-definidas positivas para garantir que ela parametrize uma métrica de distância (Bilenko et al., 2004). Isto é realizado pelo procedimento descrito em Xing et al. (2002), que consiste em decompor a matriz $\mathbf{A}_{k}=\mathbf{X}^{T} \boldsymbol{\Lambda} \mathbf{X}$, em que $\mathbf{X}$ é a matriz de autovetores de $\mathbf{A}_{k}$ e $\boldsymbol{\Lambda}$

\footnotetext{
${ }^{6}$ Neste caso, supõe-se que a distribuição gaussiana que representa cada grupo $C_{k}$ tem matriz de covariância $\mathbf{A}_{k}^{-1}$ (Bilenko et al., 2004).
} 
é a matriz diagonal com os autovalores de $\mathbf{A}_{k}$. Depois da decomposição, os autovalores em $\boldsymbol{\Lambda}$ menores que zero são substituídos por 0 , e então a matriz $\mathbf{A}_{k}$ é reconstruída.

$$
\begin{aligned}
\mathbf{A}_{k} & =\left|C_{k}\right|\left(\sum_{\mathbf{x}_{n} \in C_{k}}\left(\mathbf{x}_{n}-\mathbf{c}_{k}\right)\left(\mathbf{x}_{n}-\mathbf{c}_{k}\right)^{T}+\sum_{l \in \mathcal{V}_{\mathcal{M}, g_{\mathcal{M}}(l)=k}} \frac{1}{2} w_{l}\left(o_{\mathcal{M}}(l)-o_{\mathcal{M}}^{\prime}(l)\right)\left(o_{\mathcal{M}}(l)-o_{\mathcal{M}}^{\prime}(l)\right)^{T}\right. \\
+ & \sum_{l \in \mathcal{V}_{\mathcal{M}, g_{\mathcal{M}}^{\prime}(l)=k}} \frac{1}{2} w_{l}\left(o_{\mathcal{M}}(l)-o_{\mathcal{M}}^{\prime}(l)\right)\left(o_{\mathcal{M}}(l)-o_{\mathcal{M}}^{\prime}(l)\right)^{T} \\
+ & \left.\sum_{l \in \mathcal{V}_{\mathcal{C}}, g_{\mathcal{C}}(l)=k} \bar{w}_{l}\left(\left(\mathbf{x}_{d}^{\left(g_{\mathcal{C}}(l)\right)}-\mathbf{x}_{d^{\prime}}^{\left(g_{\mathcal{C}}(l)\right)}\right)\left(\mathbf{x}_{d}^{\left(g_{\mathcal{C}}(l)\right)}-\mathbf{x}_{d^{\prime}}^{\left(g_{\mathcal{C}}(l)\right)}\right)^{T}-\left(o_{\mathcal{C}}(l)-o_{\mathcal{C}}^{\prime}(l)\right)\left(o_{\mathcal{C}}(l)-o_{\mathcal{C}}^{\prime}(l)\right)^{T}\right)\right)^{-1}(4.13)
\end{aligned}
$$

O custo computacional de uma iteração do algoritmo MPCK-Means é $O(K \cdot M \cdot(|\mathcal{M}|+$ $\left.|\mathcal{C}|)+M^{2} \cdot(|\mathcal{M}|+|\mathcal{C}|)+K \cdot M^{3}+K \cdot N \cdot M^{2}+N^{2} \cdot M^{2}\right)$. O custo cúbico em relação ao número de atributos é proveniente do cálculo do determinante e da decomposição dos autovalores e autovetores de cada matriz $\mathbf{A}_{k}$, e o custo quadrático em relação ao número de objetos é devido à atualização do par de objetos mais distantes de acordo com cada métrica.

\subsubsection{Constrained Hybrid Genetic Algorithm}

Hong et al. (2008) propuseram um algoritmo evolutivo baseado no Pairwise Constrained K-Means ${ }^{7}$ (Bilenko et al., 2004). Diferentemente do F-EAC, o Constrained Hybrid Genetic Algorithm (Cop-HGA) é um algoritmo evolutivo para Agrupamento de Dados com Restrições (ADR). Para a codificação das partições, assim como no F-EAC, o Cop-HGA utiliza codificação inteira baseada em rótulos. Como forma de avaliar a adequabilidade de cada indivíduo é utilizada a seguinte função objetivo:

$$
J_{\text {Cop-HGA }}=\sum_{k=1}^{K} \sum_{\mathbf{x}_{n} \in C_{k}}\left\|\mathbf{x}_{n}-\mathbf{c}_{k}\right\|^{2}+\lambda \cdot\left(\left|\mathcal{V}_{\mathcal{M}}\right|+\left|\mathcal{V}_{\mathcal{C}}\right|\right),
$$

que é a mesma apresentada em Bilenko et al. (2004) considerando que o custo de violar uma restrição independe do seu tipo (ML e CL). $\mathrm{O}$ valor de $\lambda$ é ajustado a cada geração por meio de uma heurística. Em particular, considerando que $\Pi^{i}$ é a partição representada pelo $i$-ésimo indivíduo da geração atual, $\lambda$ é ajustado de acordo com a Equação 4.15, que calcula a distância média entre um objeto e o centróide do seu grupo, de tal forma que ambos os termos da função objetivo tenham a mesma importância (Hong et al., 2008).

$$
\lambda=\frac{1}{|\mathcal{P}| \cdot N} \sum_{\Pi^{i} \in \mathcal{P}} \sum_{C_{k} \in \Pi^{i}} \sum_{\mathbf{x}_{n} \in C_{k}}\left\|\mathbf{x}_{n}-\mathbf{c}_{k}\right\|^{2}
$$

É importante fazer algumas observações em relação à Equação (4.14). Primeiro, a mesma só pode ser usada quando o número de grupos é fixo, já que o primeiro termo é simplesmente a função objetivo do $K$-means, que é conhecidamente monotonicamente decrescente em relação

\footnotetext{
${ }^{7}$ Este algoritmo é similar ao MPCK-Means (descrito na Seção 4.3.1.3) porém sem o aprendizado de métrica.
} 
ao número de grupos. Além disso, não há uma boa justificativa teórica para se considerar que o custo de violar qualquer restrição é uniforme, principalmente no cenário no qual as restrições podem ser errôneas ou ruidosas.

Para a seleção dos indivíduos que irão sobreviver para a próxima geração, é utilizado elitismo e seleção por torneio (Eiben e Smith, 2003). Neste algoritmo, a seleção por torneio opera da seguinte maneira: (i) dois indivíduos são selecionados aleatoriamente da população; (ii) destes dois indivíduos, o melhor indivíduo, de acordo com o erro quadrático, é mantido para a próxima geração.

No algoritmo Cop-HGA, nenhum operador de cruzamento é utilizado. Para realizar a mutação é utilizado o operador One-step Constrained K-means apresentado no Algoritmo 12. É necessário que o usuário defina um parâmetro $(\alpha)$ que indica a probabilidade de tentar minimizar as restrições ou atender o critério de minimização do erro quadrático. Este operador não é orientado a grupos, mas, é guiado.

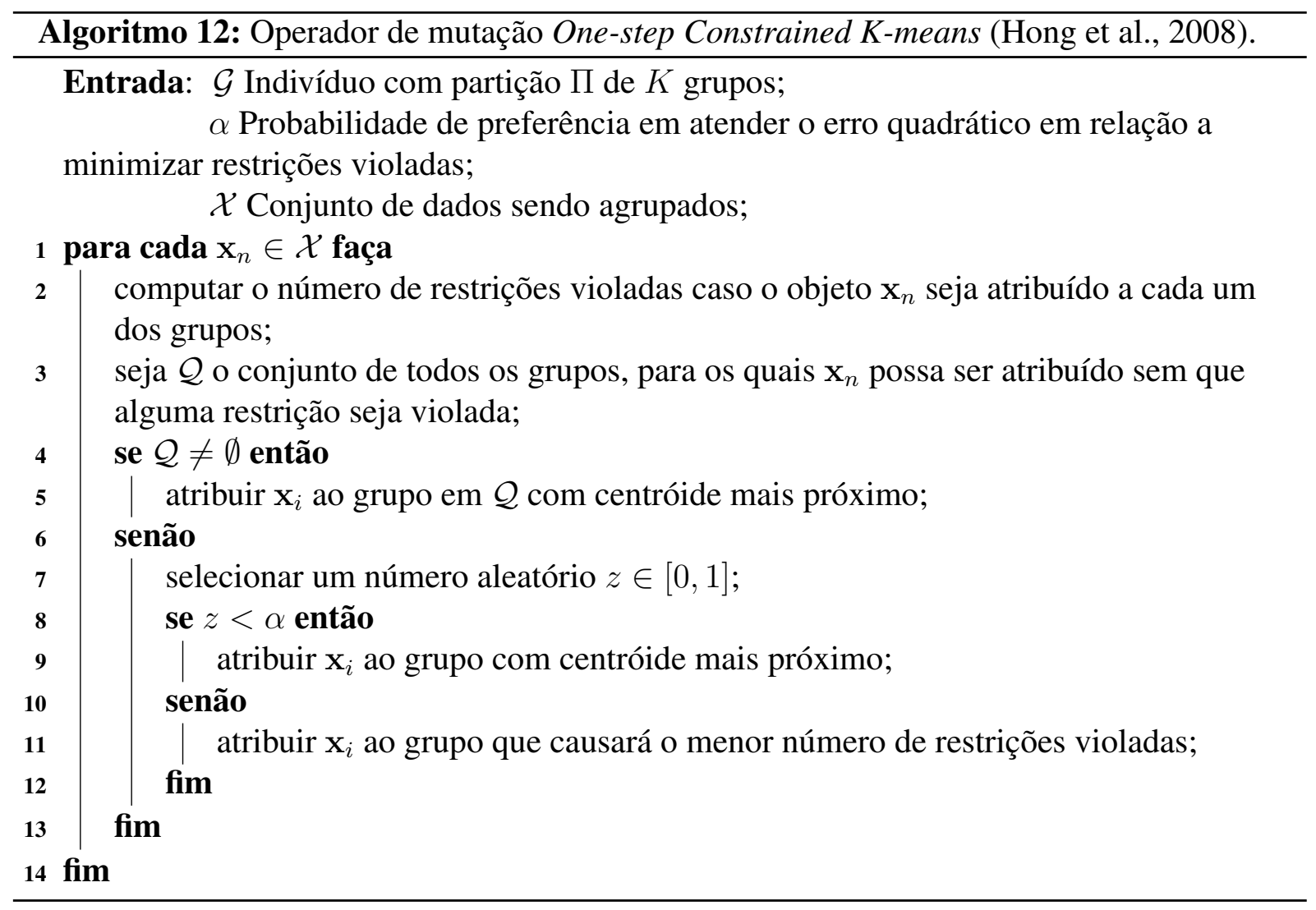

Conforme pode ser observado, o algoritmo Cop-HGA possui algumas limitações. Especificamente, é necessário que o usuário informe o número de grupos e, além disso, por não possuir uma etapa de refinamento de soluções, o algoritmo pode levar muitas gerações para convergir. Não obstante, a possível atribuição de um objeto a um grupo, considerando apenas o número de restrições violadas, pode gerar soluções ruins, dado que as distâncias entre o objeto e os grupos são desconsideradas. 


\subsubsection{Algoritmos Baseados no Expectation Maximization para Gaussian Mixture Models}

No contexto de ADR, alguns trabalhos propuseram modificações no modelo de GMM para incorporar restrições. O trabalho de Shental et al. (2003) foi o precursor desta linha e baseia-se na hipótese de amostragem dos chunklets. Especificamente, são considerados dois modelos: (i) chunklets são amostras i.i.d. em relação à probabilidade a priori da distribuição de origem (os objetos em cada chunklet também são amostras i.i.d.); (ii) objetos são amostrados i.i.d, sem o conhecimento da distribuição de origem, e apenas depois chunklets são formados a partir destes objetos. Para o modelo (i), é derivada uma solução fechada para incorporar restrições ML. No entanto, para incorporar restrições CL e para o modelo (ii), não é possível encontrar uma solução fechada, tornando necessário o uso de aproximações. Duas extensões desta abordagem são descritas por Law et al. (2005) e Lu e Leen (2007a), permitindo que restrições sejam violadas e que o usuário informe a confiança que tem em cada restrição.

Em Lange et al. (2005), é descrito um modelo diferente dos supramencionados e baseado em Markov Random Fields (Kindermann et al., 1980) e no uso de Mean field approximation (Opper e Saad, 2001). Neste modelo, é possível que o usuário indique tanto a importância dos objetos envolvidos em restrições em relação aos objetos sem restrições, quanto a importância de atender às restrições. É importante ressaltar que todas as abordagens descritas anteriormente necessitam que o usuário forneça o número de grupos (componentes da mistura) como entrada. A estimação do número de componentes é tratada no trabalho de Zhao e Miller (2005) utilizando o Bayesian Information Criterion (BIC) (Schwarz, 1978). Além disso, o algoritmo proposto procura identificar cenários em que a hipótese de um grupo por classe não é válida, permitindo que uma classe seja representada por mais de um grupo. O trabalho de Zhao e Miller (2005) foi estendido e aprimorado recentemente por Raghuram et al. (2014), incorporando uma busca incremental pelo número de grupos. Este algoritmo está intimamente relacionado ao algoritmo FIECE-EM desenvolvido nessa tese, sendo inclusive utilizado nas análises experimentais, e por conta disso, será descrito em maiores detalhes na próxima seção.

\subsubsection{Multiple Components Parameterized}

Raghuram et al. (2014) propuseram o método, denominado Multiple Components Parameterized (MCP), baseado em busca gulosa que, em conjunto com o algoritmo EM, estima o número de grupos por classe considerando que as restrições ML e CL são provenientes de classes. Inicialmente, é utilizado o OMR-EM com o critério BIC (Bayesian Information Criterion) ${ }^{8}$ considerando uma faixa de valores aceitáveis para o número de grupos para o usuário. $\mathrm{O}$ melhor modelo obtido pelo OMR-EM determina o número máximo de grupos para o processo de otimização que considera as restrições.

\footnotetext{
${ }^{8} \mathrm{O}$ critério BIC é proporcional ao MDL matematicamente, apesar de serem derivados a partir de diferentes abordagens (Koller e Friedman, 2009).
} 
Considere $\Delta_{N \times N}$ como uma matriz de confiança com pesos relativos para as restrições, i.e., $\delta_{i j}=0$ se não existe uma restrição entre os objetos $\mathbf{x}_{i}$ e $\mathbf{x}_{j}$ e $\delta_{i j} \in[0,1]$ se existe uma restrição entre os objetos. Adicionalmente, considere que existam até $L_{\max }$ classes a serem derivadas a partir das restrições, e que $\Xi_{K \times L_{\max }}$ é uma matriz binária em que $\xi_{k l}=1$ se o grupo $k$ pertence a classe $l$, e zero caso contrário. A função a ser minimizada no processo de otimização pode ser então definida como:

$$
\begin{gathered}
U(\mathcal{Z}, \Psi)=-\ln P(\mathcal{X}, \mathcal{Z} \mid \Psi)+\beta h(\mathcal{Z}, \Xi, \Delta) \\
h(\mathcal{Z}, \Xi, \Delta)=\sum_{c_{=}(i, j) \in \mathcal{M}} \delta_{i j}\left(1-\sum_{l=1}^{L_{\max }} w_{i l} w_{j l}\right)+\sum_{c_{\neq}(i, j) \in \mathcal{C}} \delta_{i j} \sum_{l=1}^{L_{\max }} w_{i l} w_{j l}, \\
w_{i l}=\sum_{k=1}^{K} z_{i k} \xi_{k l} .
\end{gathered}
$$

O primeiro termo é a função logarítmica de verossimilhança do modelo, enquanto que o segundo é a penalização por violação de restrições ponderado por um parâmetro $\beta$ definido pelo usuário. Conforme derivação apresentada em Raghuram et al. (2014), este modelo resulta em uma distribuição cujo cálculo é intratável devido à dependência entre objetos envolvidos em restrições. Para superar tal problema, os autores utilizam uma aproximação forçando uma suavização no cômputo da responsabilidade de um componente sobre um objeto.

Adicionalmente, otimizar a matriz $\Xi$ demanda um esforço computacional proibitivo devido às $L^{K}$ possíveis combinações, de classes em grupos. Para superar isso, os autores realizam um processo de otimização iterativo, realizando rotulações uma por vez até não haver mais mudanças.

O processo de otimização realiza uma busca considerando de 2 até $K_{\text {ceil }}$ grupos, sendo $K_{\text {ceil }}$ o número de grupos do modelo obtido pelo OMR-EM. Para cada número de grupos o valor da função objetivo (Equação (4.16)) é avaliado. O número de grupos é incrementado utilizando um critério de divisão de componentes. Especificamente, um componente é selecionado aleatoriamente e tem seus parâmetros divididos da seguinte forma: um dos autovetores da matriz de covariância é selecionado e as novas médias são obtidas nas direções opostas da média original considerando o autovetor; as novas matrizes de covariâncias são obtidas removendo um múltiplo deste autovetor, mantendo os demais autovetores sem modificações; os novos coeficientes de mistura são obtidos dividindo igualmente o coeficiente do componente original. Este procedimento é similar ao descrito por Blekas e Lagaris (2007) (Seção 2.3). Este processo é realizado para um número pré-fixado de grupos e a solução com maior valor de verossimilhança e cujas penalidades não são significantemente diferentes do modelo anterior é escolhido.

O algoritmo não possui premissas sobre o número de classes representadas pelas restrições, o que o permite encontrar um número de classes arbitrário ( $L_{\text {max }}$ é definido pelo usuário). No entanto, na prática, ao assumir que as restrições são derivadas de objetos rotulados o caso mais 
comum é que as novas classes sejam na verdade novos grupos de classes pré-estabelecidas.

A flexibilidade do MCP em modelar dados vem acompanhada de um custo computacional considerável. Especificamente, o custo assintótico é de $O\left(K_{\max }^{2} N M^{2}+K_{\max }^{3} N_{r} L_{\max } M^{2}\right)$, sendo $N_{r}$ o número de objetos envolvidos em restrições (Raghuram et al., 2014). Além disso, a definição do parâmetro $\beta$ não é trivial por parte do usuário, o que demanda a avaliação de diferentes valores para este parâmetro.

\subsection{Considerações Finais}

Neste capítulo, foi realizada uma revisão bibliográfica da área de Agrupamento de Dados com Restrições, tendo como foco algoritmos que incorporam as restrições no processo de agrupamento dos dados. Dentre estes algoritmos, pode-se observar que grande parte utiliza como base os algoritmos K-means e Expectation Maximization para Gaussian Mixture Model. A maioria dos trabalhos apresentados (exceto Zhao e Miller (2005) e Raghuram et al. (2014)) baseia-se nas premissas de que o número de classes é igual ao número de grupos e de que todos os dados estão disponíveis a priori para o processamento.

Nos próximos capítulos, são apresentadas propostas desenvolvidas nesta tese para tratar ambas as premissas, relaxando-as. Em particular, no Capítulo 5, são apresentados dois algoritmos que são capazes de processar os dados de forma online, não necessitando portanto que todos os dados estejam disponíveis a priori. O primeiro algoritmo é uma extensão do LCVQE (Seção 4.3.1.2) para o cenário online. A escolha do LCVQE foi motivada pelos seus resultados obtidos em uma análise empírica comparativa com o CVQE (Seção 4.3.1.1) e o MPCK-Means (Seção 4.3.1.3) publicada em Covões et al. (2013c). Estes experimentos evidenciaram que os resultados obtidos pelo algoritmo LCVQE são competitivos com os resultados obtidos pelo CVQE, violando menos restrições e sendo mais eficiente computacionalmente. Além disso, na maioria dos casos, o LCVQE apresentou melhor acurácia quando comparado com o algoritmo MPCK-Means. O segundo algoritmo é uma extensão de um algoritmo bem-conhecido na literatura de algoritmos online, denominado Rival Penalized Competitive Learning (RPCL), para o contexto de ADR.

Para tratar da questão de múltiplos grupos por classe, são feitas duas propostas nesta tese. A primeira, denominada Multiple Clusters per Class K-means (MCCK) é apresentada no Capítulo 6 , utiliza o algoritmo $K$-means. No Capítulo 7 é apresentada uma outra proposta que baseia-se no EM para GMM, denominada Feasible-Infeasible Evolutionary Create \& Eliminate Algorithm for Expectation Maximization (FIECE-EM), que pode ser vista como uma extensão e generalização do algoritmo MCCK. 


\section{CAPÍTULO}

\section{Aprendizado Competitivo para Agrupamento de Dados com Restrições}

\subsection{Considerações Inciais}

Algoritmos de aprendizado competitivo são caracterizados pela competição entre $K$ neurônios (protótipos) (Choy e Siu, 1998). A cada passo, uma entrada (objeto) $\mathbf{x}_{n}$ é apresentada, e o neurônio que vence a competição aprende tal entrada, i.e., o neurônio é adaptado na direção de $\mathbf{x}_{n}$ com uma taxa de aprendizado especificada a priori. Esta abordagem é conhecida como Winner-Take-All (WTA) (Hofmann e Buhmann, 1998), e tem sido estudada extensivamente (Choy e Siu, 1998; Patra, 2011). Esta abordagem é diferente da realizada em algoritmos de processamento em lote, nos quais todos os dados são percorridos antes de uma atualização do modelo ser realizada.

O aprendizado competitivo pode ser visto como o particionamento do espaço de atributos (Hofmann e Buhmann, 1998; Chen et al., 2012; Silva e Liang, 2012). Especificamente, considerando os neurônios como protótipos de grupos, e utilizando a distância euclidiana como medida de competição, a abordagem WTA pode ser vista como uma versão online do algoritmo $K$-means. Em uma visão mais geral, algoritmos de aprendizado competitivo pertencem à classe de algoritmos de gradiente descendente estocásticos (Patra, 2011).

O uso de supervisão em algoritmos de aprendizado competitivo foi considerado no algoritmo LVQ (Kohonen, 1990), em que assume-se que os rótulos dos objetos da base de treinamento são conhecidos. Estes rótulos são usados como guia para refinar as regiões de fronteira de decisão. No entanto, a aquisição desses rótulos costuma ser cara, enquanto que dados nãorotulados costumam ser abundantes em diversas aplicações. Portanto, algoritmos capazes de usar apenas supervisão parcial - e.g., na forma de um subconjunto de objetos rotulados ou de 
restrições sobre alguns pares de objetos — são necessários. Algoritmos online podem ser úteis para análise de dados em larga escala, bem como para o uso de restrições em cenários distribuídos e assíncronos (Patra, 2011). Outra característica atrativa de algoritmos online é que eles são apropriados para cenários em que a base de dados $(\mathcal{X})$ sofre mudanças (adição/remoção de objetos), bem como para cenários em que o conjunto de restrições está mudando constantemente.

Neste capítulo, são introduzidos dois algoritmos online de Agrupamento de Dados com Restrições (ADR). O primeiro, denominado Online Linear Constrained Vector Quantization Error (O-LCVQE), é uma adaptação do algoritmo $\mathrm{LCVQE}^{1}$ para a abordagem de aprendizado competitivo. A motivação em adaptar o algoritmo LCVQE vem de seus bons resultados empíricos, conforme apresentado em Covões et al. (2013c). O segundo algoritmo, baseado no bem-conhecido Rival Penalized Competitive Learning (RPCL) (Xu et al., 1993) é denominado C-RPCL, e incorpora as restrições buscando não afetar a simplicidade do método original.

\subsection{Aprendizado Competitivo}

O algoritmo de aprendizado competitivo mais simples é baseado na abordagem WTA, em que o protótipo (neurônio) mais próximo da entrada se move na direção da entrada com uma taxa de aprendizado $\alpha$, i.e., $\Delta \mathbf{c}_{k}=r_{k} \alpha\left(\mathbf{x}_{n}-\mathbf{c}_{k}\right), n \in\{1, \ldots, N\}, k \in\{1, \ldots, K\}$, sendo $r_{k}$ igual a 1 se $\mathbf{c}_{k}$ é o protótipo mais próximo de $\mathbf{x}_{n}$, e 0 caso contrário. Apesar de sua simplicidade, WTA apresenta um problema conhecido como sub-utilização de neurônios (Choy e Siu, 1998), que ocorre devido a alguns neurônios não serem inicializados apropriadamente e consequentemente não serem capazes de ganhar nenhuma competição. Por exemplo, se todos os neurônios forem inicializados em uma região longe dos dados, é provável que o vencedor da primeira entrada também será o vencedor das demais competições.

Diversos algoritmos competitivos foram desenvolvidos para tratar o problema da sub-utilização de neurônios - uma discussão detalhada sobre o assunto pode ser encontrada em Choy e Siu (1998). Uma abordagem simples é considerar a frequência de vitória de cada protótipo, i.e., ponderando as distâncias entre protótipos e objetos pela frequência de vitórias de cada neurônio. Esta abordagem é conhecida como aprendizado competitivo sensível à frequência (Ahalt et al., 1990). Uma extensão desta abordagem, que pode ser vista como uma extensão do algoritmo LCVQ2 (Kohonen, 1990), é o Rival Penalized Competitive Learning (RPCL) (Xu et al., 1993). Neste algoritmo, o segundo protótipo mais próximo da entrada, denominado rival, é penalizado sendo movido na direção contrária do objeto. Esta penalização é proporcional a uma diferente taxa de aprendizado $(\beta)$ - usualmente $\beta \ll \alpha$. Para simplificar, o termo taxa de esquecimento será usado para se referir a $\beta$.

Na sequência, são descritos os algoritmos de aprendizado competitivo com restrições, denominados de Online Linear Constrained Vector Quantization Error (O-LCVQE) e Constrained Rival Penalized Competitive Learning (C-RPCL). Conforme discutido na Seção 4.2, quando

\footnotetext{
${ }^{1}$ Seção 4.3.1.2.
} 
restrições são extraídas de rótulos de classes, as restrições podem ser prejudiciais ao agrupamento dos dados, pois classes podem corresponder a mais de um grupo (i.e., classes multimodais). Especificamente, restrições ML envolvendo objetos que pertençam à mesma classe mas a grupos distintos irá guiar o algoritmo de agrupamento para a união desses grupos. Portanto, para evitar esse possível viés, nos algoritmos online aqui apresentados, são consideradas apenas restrições CL, que por sua vez, são válidas independentemente do número de grupos por classe. No caso em que as restrições ML podem ser assumidas como válidas, incorporá-las nos algoritmos propostos não é um problema.

\subsection{Online Linear Constrained Vector Quantization Error}

Online Linear Constrained Vector Quantization Error (O-LCVQE) também pode ser visto como uma abordagem WTA capaz de lidar com restrições CL. O procedimento para tratar restrições CL é similar ao procedimento do LCVQE, i.e., o protótipo vencedor é definido como no LCVQE. Especificamente, se a entrada está envolvida em uma restrição CL, e o mesmo protótipo $\left(\mathbf{c}_{k}\right)$ é o mais próximo dos dois objetos em questão, então dois casos são avaliados: (i) usar o mesmo protótipo para representar ambos os objetos; (ii) ajustar o segundo protótipo mais próximo $\left(\mathbf{c}_{k_{1}}\right)$ para o objeto da restrição mais distante de $\mathbf{c}_{k}$. Cada caso tem um custo associado, que é oriundo da função objetivo do LCVQE - Eq. (4.5). Depois de computados, o caso com menor custo é escolhido. Para o segundo caso, se o objeto mais distante não é a entrada atual $\left(\mathbf{x}_{n}\right)$, um passo adicional é realizado para que $\mathbf{c}_{k}$ seja movido na direção contrária à do objeto mais distante e na direção de $\mathbf{x}_{n}$. Os passos principais do O-LCVQE são apresentados no Algoritmo 13.

Note que o algoritmo lida com restrições CL de uma maneira gulosa. Em particular, para cada restrição são avaliadas todas as combinações de rotulações considerando apenas os custos impostos pela restrição - sem considerar o custo que tal restrição pode causar a outras restrições relacionadas à entrada atual. Este procedimento é relacionado com a abordagem de aprendizado competitivo, dado que as restrições são sempre processadas de forma online. Além disso, quando nenhuma restrição é fornecida, o algoritmo se reduz à abordagem WTA (Seção $5.2)$.

\subsection{Constrained Rival Penalized Competitive Learning}

O algoritmo Rival Penalized Competitive Learning (RPCL) (Xu et al., 1993) também pode ser modificado para lidar com restrições CL. A intuição que norteia o algoritmo resultante desta modificação, denominado Constrained Rival Penalized Competitive Learning (C-RPCL), é que se uma restrição CL é violada ao rotular um objeto a um determinado protótipo, então busca-se o protótipo rival mais próximo que não causa nenhuma violação de restrições. Este rival mais próximo se torna o vencedor, e o protótipo vencedor anterior é movido na direção 


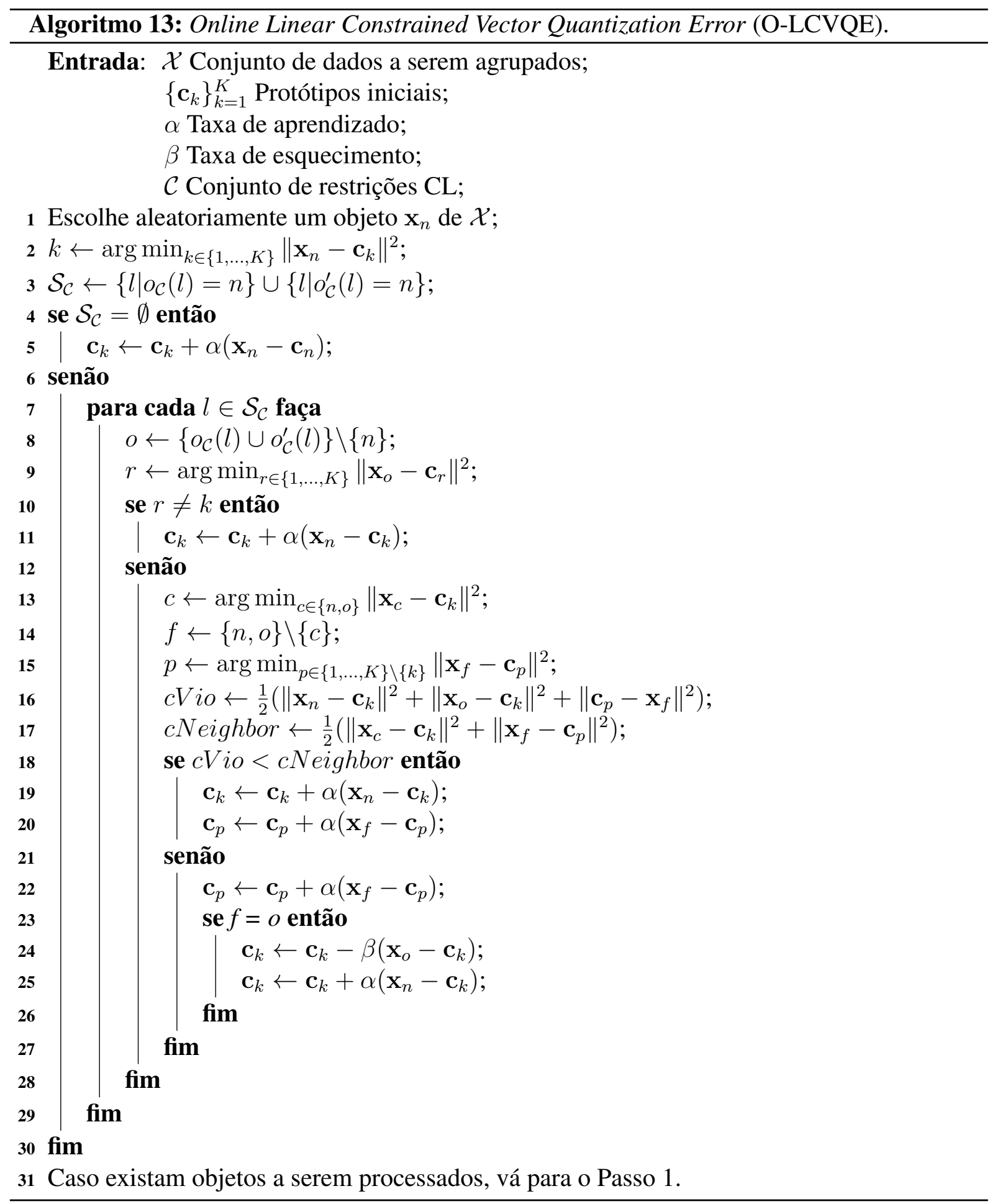


contrária àquele objeto. Para o caso degenerado em que nenhum rival válido seja encontrado, o procedimento padrão do RPCL é utilizado.

A variável $\gamma$ é introduzida para evitar a sub-utilização de neurônios (protótipos) mencionada na Seção 5.2. Especificamente, $\gamma$ armazena a contagem (normalizada) de vitórias de cada protótipo. A distância entre objetos e protótipos é ponderada por $\gamma$, de forma que vencedores frequentes anteriores tenham menos chances de vencer as competições subsequentes. Os principais passos do C-RPCL são descritos no Algoritmo 14. Diferentemente do O-LCVQE, o C-RPCL leva em conta todas as restrições disponíveis para definir o protótipo vencedor e o protótipo rival. Desta forma, C-RPCL pode evitar algumas atualizações desnecessárias dos protótipos que seriam feitas pelo O-LCVQE. Quando nenhuma restrição é fornecida, C-RPCL reduz ao RPCL, que por sua vez, é capaz de estimar o número de grupos. O uso das restrições não faz com que o C-RPCL perca esta característica.

\subsection{Avaliação Empírica}

Para comparar os algoritmos O-LCVQE e C-RPCL, foram realizados experimentos em oito bases de dados comumente utilizadas como benchmarks. A maioria delas está disponível no bem-conhecido repositório da UCI (Asuncion e Newman, 2007). Adicionalmente, foi utilizada a base de dados 9Gauss (Campello et al., 2009), que é formada por nove grupos balanceados distribuídos de acordo com distribuições gaussianas que possuem certo grau de sobreposição. Seguindo Bilenko et al. (2004), foram utilizadas as bases de dados Letters e Pendigits apenas com as classes I, J e L e 3, 8 e 9, respectivamente. De acordo com Bilenko et al. (2004) estas classes representam problemas de classificação difíceis. As principais características das bases de dados são sumarizadas na Tabela 5.1.

Conforme mencionado na Seção 5.1, considera-se que as restrições são oriundas de rótulos de classes. Para gerar estas restrições são considerados casos em que amostras de objetos rotulados estão disponíveis. Em particular, diferentes quantidades de objetos rotulados foram adotadas - i.e., 5, 10, 15 e 20 objetos foram selecionados aleatoriamente de cada classe. Destas amostras, todas as possíveis restrições CL foram deduzidas. Por exemplo, para a base de dados Iris, que possui três classes, amostrando 5 objetos por classe um conjunto de 75 restrições foi obtido. Esta metodologia simula um cenário em que um especialista de domínio fornece os rótulos de apenas alguns objetos. Neste cenário, é desejável usar o máximo de informação possível dos objetos rotulados.

Cada algoritmo foi executado por 100 épocas (passagens completas pelos dados). Protótipos iniciais foram amostrados de uma distribuição gaussiana, com média e covariância estimadas a partir de $20 \%$ dos dados. Devido à sensibilidade à inicialização e à ordem de processamento dos objetos, cada algoritmo foi executado cinco vezes com diferentes protótipos iniciais e ordens de processamento. Todo o processo foi repetido 10 vezes para que melhores estimativas das estatísticas de interesse fossem obtidas. Como feito por Xu et al. (1993), a taxa de aprendizado 


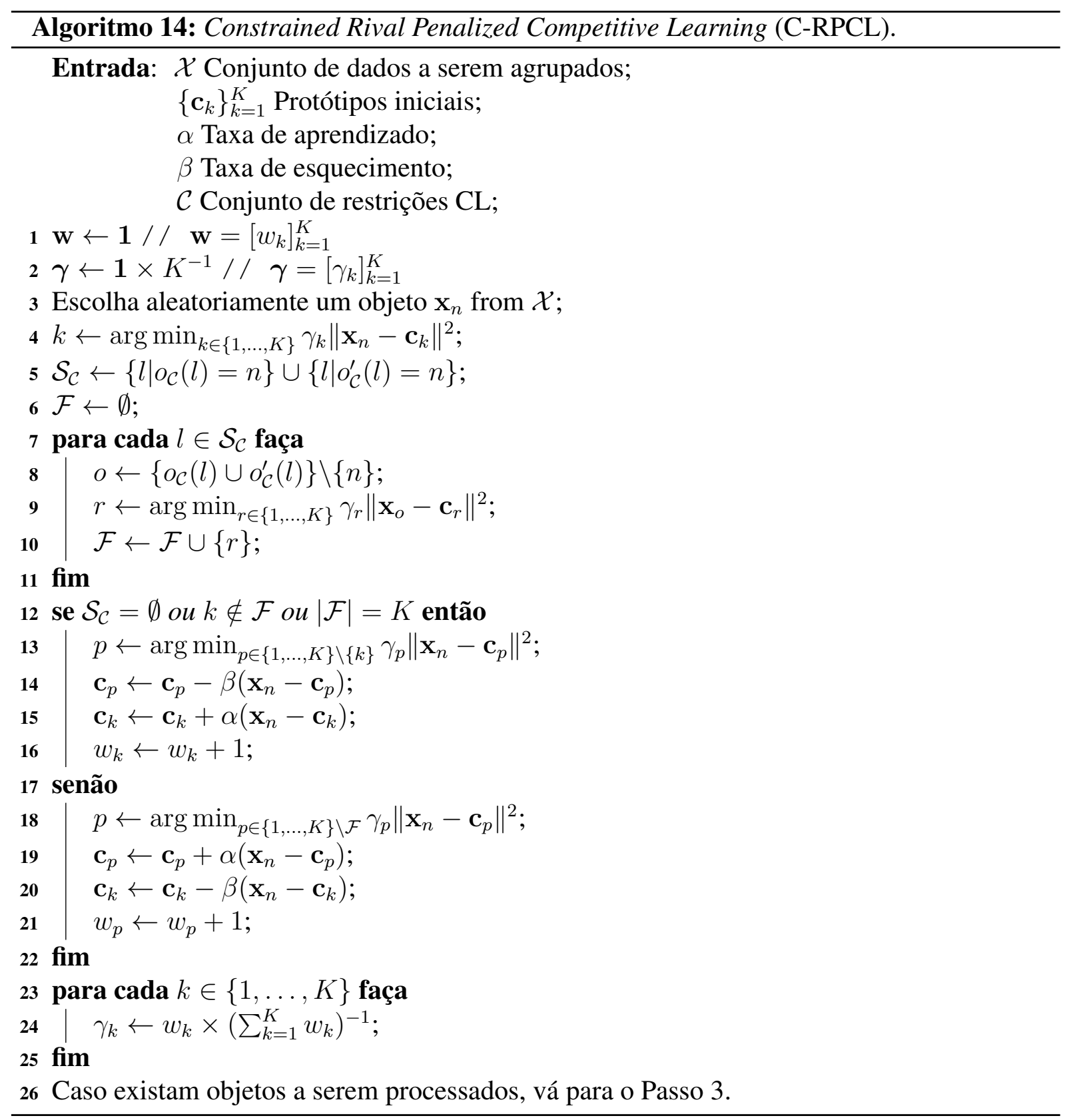




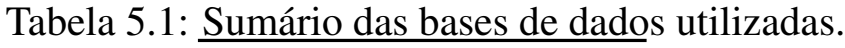

\begin{tabular}{cccc}
\hline Nome & $N$ & $M$ & $K$ \\
\hline 9Gauss & 900 & 2 & 9 \\
Ionosphere & 351 & 34 & 2 \\
Iris & 150 & 4 & 3 \\
Wine & 178 & 13 & 3 \\
Breast Cancer & 683 & 9 & 2 \\
Pendigits & 3165 & 16 & 3 \\
Letters & 2263 & 16 & 3 \\
Pima & 768 & 8 & 2 \\
\hline
\end{tabular}

e esquecimento foram definidas como 0.05 e 0.002 , respectivamente.

Tabela 5.2: Diferenças entre valores de NMI obtidos pelo algoritmo C-RPCL e o LCVQE (quanto maior melhor) — \#OR significa número de objetos rotulados usados para gerar as restrições.

\begin{tabular}{|c|c|c|c|c|c|c|}
\hline Algoritmo & RPCL & \multicolumn{5}{|c|}{ C-RPCL } \\
\hline Base de dados \# OR & $\mathbf{0}$ & 5 & 10 & 15 & 20 & Média \\
\hline 9Gauss & $-0,010$ & 0,005 & $-0,015$ & $-0,005$ & $-0,007$ & $-0,006$ \\
\hline Ionosphere & $-0,010$ & 0,004 & $-0,004$ & 0,000 & 0,017 & 0,001 \\
\hline Iris & $-0,007$ & $-0,015$ & 0,001 & $-0,037$ & $-0,019$ & $-0,015$ \\
\hline Wine & 0,003 & 0,008 & 0,030 & 0,035 & 0,038 & 0,023 \\
\hline Breast Cancer & 0,000 & 0,016 & $-0,011$ & 0,007 & 0,027 & 0,008 \\
\hline Pendigits & 0,204 & 0,184 & 0,227 & 0,280 & 0,250 & 0,229 \\
\hline Letters & 0,013 & 0,010 & 0,000 & 0,008 & 0,020 & 0,010 \\
\hline Pima & 0,001 & 0,000 & $-0,011$ & $-0,005$ & 0,001 & $-0,003$ \\
\hline
\end{tabular}

Tabela 5.3: Diferenças entre valores de NMI obtidos pelo algoritmo O-LCVQE e o LCVQE (quanto maior melhor) — \#OR significa número de objetos rotulados usados para gerar as restrições.

\begin{tabular}{|c|c|c|c|c|c|c|}
\hline Algoritmo & WTA & \multicolumn{5}{|c|}{ O-LCVQE } \\
\hline Base de dados $\#$ OR & $\mathbf{0}$ & 5 & 10 & 15 & 20 & Média \\
\hline 9Gauss & $-0,017$ & $-0,032$ & $-0,101$ & $-0,100$ & $-0,102$ & $-0,071$ \\
\hline Ionosphere & $-0,010$ & $-0,007$ & $-0,028$ & $-0,077$ & $-0,057$ & $-0,036$ \\
\hline Iris & 0,000 & 0,013 & 0,010 & $-0,027$ & $-0,024$ & $-0,006$ \\
\hline Wine & 0,000 & 0,012 & 0,020 & 0,025 & 0,028 & 0,017 \\
\hline Breast Cancer & $-0,018$ & 0,016 & 0,013 & $-0,023$ & $-0,013$ & $-0,005$ \\
\hline Pendigits & 0,052 & 0,031 & 0,173 & 0,128 & 0,111 & 0,099 \\
\hline Letters & $-0,006$ & 0,000 & 0,010 & 0,024 & 0,052 & 0,016 \\
\hline Pima & $-0,003$ & $-0,004$ & $-0,019$ & $-0,027$ & $-0,019$ & $-0,014$ \\
\hline
\end{tabular}

A qualidade das partições obtidas foi avaliada por meio do Normalized Mutual Information (NMI) (Strehl e Ghosh, 2003) levando em consideração a partição de referência fornecida pelas classes/grupos conhecidos. Como baseline de comparação² ${ }^{2}$ é considerado o algoritmo LCVQE (Pelleg e Baras, 2007). Visto que algoritmos de processamento em lote têm acesso a mais informação para realizar a atualização dos protótipos do que algoritmos online, é esperado que suas partições sirvam de aproximações para aqueles obtidos por algoritmos de processamento em lote. Portanto, a acurácia dos algoritmos propostos é avaliada por meio das diferenças entre os valores de NMI das partições obtidas pelos algoritmos online e o LCVQE.

\footnotetext{
${ }^{2}$ Note que este é um baseline bastante competitivo, visto os resultados obtidos pelo LCVQE na comparação empírica apresentada em Covões et al. (2013c).
} 
Tabela 5.4: Médias e desvios-padrão dos valores de NMI para diferentes quantidades de objetos rotulados $(\# \mathrm{OR})$ - Pendigits.

\begin{tabular}{cccc}
\hline \# OR & C-RPCL & O-LCVQE & LCVQE \\
\hline 0 & $0.69(0.00)$ & $0.53(0.08)$ & $0.48(0.00)$ \\
5 & $0.68(0.00)$ & $0.52(0.04)$ & $0.49(0.05)$ \\
10 & $0.71(0.02)$ & $0.66(0.05)$ & $0.49(0.06)$ \\
15 & $0.76(0.00)$ & $0.61(0.07)$ & $0.48(0.06)$ \\
20 & $0.77(0.00)$ & $0.63(0.13)$ & $0.52(0.09)$ \\
\hline
\end{tabular}

Tabela 5.5: Diferenças entre o número de restrições violadas pelos algoritmos online e o LCVQE (quanto menor melhor).

\begin{tabular}{|c|c|c|c|c|c|c|c|c|c|c|}
\hline Algoritmo & \multicolumn{5}{|c|}{ C-RPCL } & \multicolumn{5}{|c|}{ O-LCVQE } \\
\hline Base de dados $\quad$ \# OR & 5 & 10 & 15 & 20 & Média & 5 & 10 & 15 & 20 & Média \\
\hline 9Gauss & 10.8 & 39.8 & 75.8 & 108.5 & 58.7 & 12.6 & 115.2 & 225.4 & 398.6 & 188.0 \\
\hline Ionosphere & 1.1 & -8.8 & -9.3 & -45.6 & -15.7 & 1.5 & -7.6 & 9.9 & 26.6 & 7.6 \\
\hline Iris & 5.6 & 17.3 & 42.7 & 51.3 & 29.2 & 3.4 & 9.7 & 35.3 & 57.7 & 26.5 \\
\hline Wine & 4.5 & 7.1 & 6.4 & 1.3 & 4.8 & 3.8 & 5.0 & -6.1 & 94.5 & 24.3 \\
\hline Breast Cancer & 2.0 & 1.4 & 14.4 & -22.0 & -1.0 & 2.0 & -3.4 & 14.2 & 4.2 & 4.2 \\
\hline Pendigits & -6.5 & -22.8 & -81.9 & -108.8 & -55.0 & 10.1 & -16.6 & -43.0 & -7.7 & -14.3 \\
\hline Letters & 4.3 & 9.6 & 22.0 & 19.6 & 13.9 & 4.8 & 15.7 & 11.6 & 16.1 & 12.1 \\
\hline Pima & 3.8 & 7.2 & 12.4 & 13.8 & 9.3 & 4.2 & 4.2 & -6.2 & -5.0 & -0.7 \\
\hline
\end{tabular}

As diferenças médias entre os valores de NMI obtidos pelos algoritmos online e os obtidos pelo LCVQE são reportados nas Tabelas 5.2 e 5.3. Um número positivo significa que o algoritmo online obteve resultados melhores que o LCVQE. Pode-se observar que o C-RPCL obteve os melhores resultados, com valores de NMI iguais ou melhores do que os observados pelo LCVQE em mais de 67\% dos casos (27 de 40). Para o O-LCVQE os resultados foram menos favoráveis - especificamente, os resultados obtidos foram iguais ou melhores que o LCVQE em apenas $47 \%$ dos casos. Comparando os algoritmos online entre si, o C-RPCL obteve melhores resultados que o O-LCVQE em $75 \%$ dos casos.

Os resultados na base de dados Pendigits chamam a atenção. Nesta base de dados, o C-RPCL obteve a maior diferença positiva observada (0,229 em média). Para entender melhor este resultado, os valores médios de NMI de cada algoritmo são apresentados na Tabela 5.4, em que dois aspectos se destacam. Primeiramente, a pequena variância nos resultados obtidos pelo C-RPCL indica certo grau de robustez em relação a inicialização dos protótipos. Segundo, para todas as quantidades de objetos rotulados as partições obtidas pelo C-RPCL apresentam valores altos de NMI ( $\approx 0,72$ em média). Estes resultados sugerem que o C-RPCL não apenas obteve os melhores resultados entre os algoritmos avaliados, mas também apresentou boas partições dos dados.

A Tabela 5.5 apresenta a diferença média entre o número de restrições violadas pelos algoritmos online em relação ao LCVQE. Neste caso, números menores são melhores, indicando que a versão online violou menos restrições que o LCVQE. A partir destes resultados, é possível notar que, como esperado, o LCVQE, que pode considerar todos os dados ao mesmo tempo, viola menos restrições do que os algoritmos online em $75 \%$ dos dados (24 de 32). No entanto, em duas bases de dados, Ionosphere e Pendigits, C-RPCL violou menos restrições que o LCVQE. 


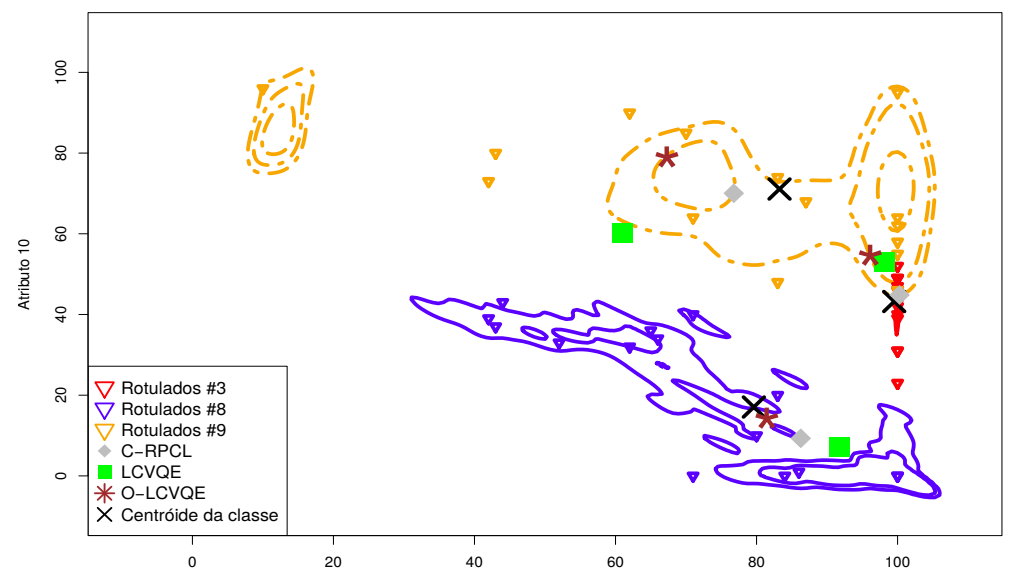

(a) Protótipos, objetos rotulados e classes - utilizando os dois atributos mais relevantes.

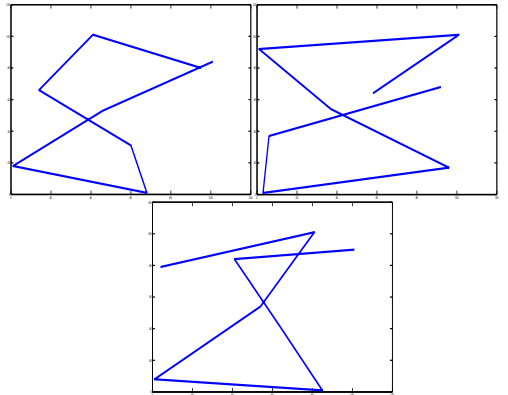

(b) Três objetos colocados no grupo da classe " 8 " pelo C-RPCL — apenas o C-RPCL classificou corretamente estes objetos.

Figura 5.1: Resultados detalhados na base de dados Pendigits.

Para compreender melhor os resultados obtidos na base de dados Pendigits, foram realizados experimentos utilizando apenas seus (dois) atributos mais relevantes - com as mesmas restrições derivadas da amostra de 15 objetos rotulados dos experimentos anteriores. Os dois atributos foram selecionados usando o bem-conhecido Nä̈ve Bayes Wrapper, que fornece um erro de classificação de 7\% (em validação cruzada de 10 pastas). Note que isso não é um procedimento prático. Pelo contrário, o uso dos rótulos de classes conhecidos para a seleção de atributos só é justificada pelo interesse em entender melhor os resultados. Sob essa perspectiva, a Figura 5.1(a) ilustra a partição obtida pela execução com a maior diferença de NMI entre o C-RPCL e o LCVQE. Os protótipos dos grupos e centróides das classes, bem como os objetos usados para derivar as restrições (apresentados como triângulos invertidos), são ilustrados. A densidade de cada classe é mostrada por seus contornos. Pode-se notar que as classes possuem sobreposição neste sub-espaço e que a classe que representa o número "9" possui um pequeno grupo ao lado esquerdo da região densa de dados. A partir dos protótipos dos grupos, pode-se notar que o C-RPCL foi menos afetado por tal grupo outlier, o que o permitiu obter uma melhor estimativa da média do grupo que o LCVQE. Por essa razão, a partição obtida pelo LCVQE tem mais erros para os objetos das classes " 8 " comparado ao C-RPCL. Para melhor ilustrar isto, na Figura 5.1(b) três objetos da classe "8" que são corretamente classificados apenas pelo C-RPCL são apresentados — os demais algoritmos erram a classificação destes objetos. É possível notar que o objeto na parte superior-esquerda é um " 8 " razoavelmente bem-formado, enquanto que os demais são difíceis de classificar. Lembrando que o reconhecimento de dígitos é um problema difícil, a capacidade de identificar corretamente números deformados (como os apresentados na Figura 5.1(b)) é relevante. Além disso, especula-se que a estrutura observada no subespaço apresentado na Figura 5.1(a) é, até certo ponto, similar à encontrada no espaço completo de 16-dimensões.

Para comparar algoritmos online com algoritmos de processamento em lote em uma base de 
Tabela 5.6: Resultados obtidos na base de dados do KDDCUP'99 de Detecção de intrusão em redes (desvios padrão em parênteses).

\begin{tabular}{ccc}
\hline Algoritmo & NMI & Tempo (segundos) \\
\hline C-RPCL & $0,82(0,03)$ & $40,2(0,2)$ \\
O-LCVQE & $0,84(0,02)$ & $28,5(0,2)$ \\
LCVQE & $0,83(0,02)$ & $121,2(26,6)$ \\
\hline
\end{tabular}

dados com um número alto de objetos, foram realizados experimentos com a base de dados do KDDCUP'99 de Detecção de intrusão em redes, comumente utilizada para avaliar algoritmos de agrupamento de streams (Aggarwal et al., 2003). Apenas classes com pelo menos 1.000 objetos foram consideradas, reduzindo o problema de 23 para 8 tipos de conexões. Esta base de dados é formada por 492.368 objetos representados por 34 atributos. Foram geradas restrições utilizando 20 objetos rotulados por classe e os experimentos foram repetidos 10 vezes. Comparações de performance são sumarizadas na Tabela 5.6. Os algoritmos online foram executados como se operando sob streams, i.e., eles executaram apenas uma passagem pelos dados (época). Note que esta aplicação específica requer processamento em tempo real, o que torna algoritmos online ainda mais apropriados. Na Tabela 5.6, pode-se notar que os valores de NMI obtidos pelos algoritmos são similares. No entanto, o tempo de computação necessário para os algoritmos online é significativamente menor do que do algoritmo de processamento em lote. Mais especificamente, o C-RPCL usou aproximadamente 33\% do tempo de processamento necessário pelo LCVQE, enquanto que o O-LCVQE usou aproximadamente $23 \%$ da mesma quantidade de tempo.

\subsection{Considerações Finais}

Neste capítulo, foram apresentados dois algoritmos de aprendizado competitivo para ADR: online, especificamente o Constrained Rival Penalized Competitive Learning (C-RPCL) e Online Linear Constrained Vector Quantization Error (O-LCVQE). Os resultados experimentais indicam que o algoritmo C-RPCL não apenas apresenta melhores resultados que o O-LCVQE, mas também pode fornecer partições melhores que o algoritmo de processamento em lote LCVQE (Pelleg e Baras, 2007). Resultados em uma base de dados com número de objetos alto evidenciaram as vantagens computacionais no uso de algoritmos online.

O material apresentado nesse capítulo foi publicado em Covões et al. (2013a). Possíveis desdobramentos para o trabalho iniciado com o desenvolvimento destes algoritmos são descritos na Seção 8.1. 


\section{CAPÍTULO}

\section{Modelando Múltiplos Grupos por Classe}

\subsection{Considerações Iniciais}

A forma mais usual de avaliar algoritmos de Agrupamento de Dados com Restrições (ADR) na literatura é por meio da geração de restrições ML e CL de rótulos de classe. No entanto, conforme mencionado na Seção 4.2, é necessário fazer uma ressalva sobre a informação fornecida pelos rótulos de classes, particularmente devido à diferença (por vezes, sutil) entre rótulos de classes e grupos. Como classes podem ser formadas por múltiplos grupos, o uso de informações extraídas de rótulos de classes para guiar algoritmos de agrupamento podem ser enganadoras. Especificamente, se dois objetos pertencem à mesma classe mas a diferentes grupos, a restrição ML entre eles irá guiar o algoritmo de agrupamento a unir esses dois grupos. É fácil notar que isso é prejudicial ao processo de agrupamento. Além disso, pode-se verificar que restrições CL ainda são válidas, contanto que a premissa de grupos $^{1}$, que supõe que objetos no mesmo grupos são provavelmente provenientes da mesma classe (Chapelle et al., 2010), seja válida. Sucintamente, o uso de rótulos de classe, como se eles fossem rótulos de grupos, implica em uma premissa de um grupo por classe. Apesar dessa premissa ser empregada frequentemente, raramente ela é colocada explicitamente em estudos de ADR, o que pode causar enganos. Para evitar tal confusão, neste capítulo as restrições extraídas de rótulos de classe são denotadas por $\overline{M L}$ e $\overline{C L}$, i.e., uma restrição $\overline{M L}$ indica que dois objetos são da mesma classe, enquanto que uma restrição $\overline{C L}$ indica que dois objetos pertencem a classes diferentes.

A validade da premissa de um grupo por classe não costuma ser verificada nos estudos experimentais reportados na literatura. Caso esta premissa não seja satisfeita, algoritmos baseados nela apresentam o efeito outlier (Klein et al., 2002), especificamente: apesar das restrições

\footnotetext{
${ }^{1}$ A premissa de grupos (cluster assumption) é comumente encontrada em diversos trabalhos da literatura de aprendizado semissupervisionado (Chapelle et al., 2010).
} 
serem satisfeitas, seus efeitos não são propagados no espaço. Para ilustrar tal situação, considere a Figura 6.1(a) (reproduzida de Klein et al. (2002)), que apresenta uma base de dados pedagógica e duas restrições ML (linhas conectando os pontos). Algumas observações sobre essa figura: (i) os objetos são distribuídos em quatro grupos; (ii) as restrições ML conectam objetos que pertencem a grupos distintos; (iii) considerando que objetos conectados por uma restrição ML deveriam estar no mesmo grupo, e que objetos similares deveriam permanecer também no mesmo grupo, a partição (apropriadamente) obtida (de acordo com tais restrições) é dada pela Figura 6.1(b). Esta partição satisfaz às restrições, mas não distribui seu efeito na vizinhança dos objetos relacionados. Neste exemplo, pode-se observar que as restrições não estão de acordo com a disposição espacial dos dados (obtida via inspeção visual). Existem duas razões principais para isso acontecer: (i) as restrições são, na verdade, restrições $\overline{M L}$, i.e., elas foram derivadas de rótulos de classes; (ii) o domínio do qual as restrições foram obtidas não está de acordo com os atributos (valores dos objetos) sendo considerados. Neste capítulo, e no próximo, são desenvolvidos algoritmos capazes de levar em conta restrições obtidas a partir de rótulos de classes, permitindo identificar a partição na Figura 6.1(c), na qual existem dois grupos por classe.

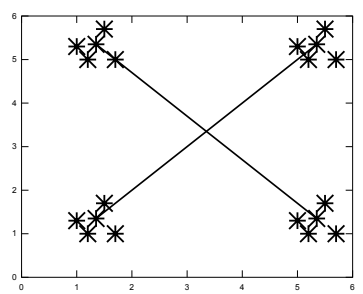

(a) Objetos/restrições

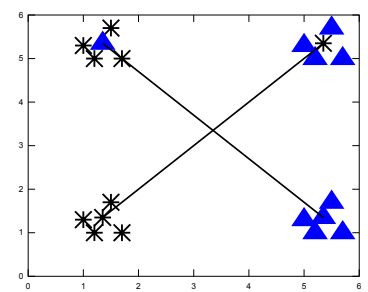

(b) Partição obtida

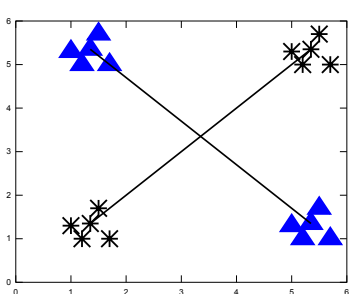

(c) Partição correta

Figura 6.1: Exemplo do efeito outlier Klein et al. (2002).

O algoritmo descrito neste capítulo para tratar o problema de múltiplos grupos por classe é denominado Multiple Clusters per Class K-means (MCCK) ${ }^{2}$ (Sestaro et al., 2012). O número de grupos é estimado para cada classe considerando tanto as distâncias entre objetos quanto restrições $\overline{C L}$ e $\overline{M L}$. O MCCK pode ser usado para análise exploratória de dados de forma a obter uma melhor sumarização dos dados se comparado à obtida de forma não supervisionada, bem como para ajudar a identificar diferentes padrões comuns a objetos da mesma classe. Por exemplo, em aplicações de diagnósticos médicos, diferentes doenças podem ser representadas por meio de classes distintas, e para cada doença/classe pode haver mais de um grupo, cada um correspondendo a uma variante da doença sendo analisada.

\footnotetext{
${ }^{2}$ Este algoritmo foi desenvolvido em conjunto com Davidson de Melo Sestaro.
} 


\subsection{Descrição do Algoritmo}

O algoritmo proposto é baseado na premissa de que restrições são definidas em relação a um conceito mais abstrato do que o de grupos. Especificamente, grupos são usualmente definidos em termos de similaridades no espaço de atributos. No entanto, em diversas aplicações, restrições ML e CL são derivadas de rótulos de classes, ou fornecidas por especialistas de domínio que normalmente não possuem conhecimento sobre a distribuição espacial dos dados. Portanto, nestas aplicações, é esperado que a premissa de um grupo por classe tenha poucas chances de ser válida.

As premissas fundamentais do MCCK são: (i) existe uma bijeção entre classes e chunklets $^{3}$; (ii) se um objeto está em um restrição $\overline{C L}$, ele está envolvido em pelo menos uma restrição $\overline{M L}$. Embora essas possam ser consideradas premissas fortes, pode-se verificar que elas são válidas se todas as restrições forem derivadas de objetos rotulados ${ }^{4}$ e se existirem pelo menos dois objetos rotulados de cada classe. Com tais premissas e com o conjunto de restrições estendido (e.g., pela regra de transitividade - Seção 4.2), é garantido que cada objeto envolvido em uma restrição também é um membro de um chunklet.

É comum inicializar algoritmos de ADR utilizando chunklets. Por exemplo, em Wagstaff et al. (2001); Bilenko et al. (2004) os protótipos são inicializados pelo centróide do chunklet. Se a premissa de um grupo por classe não é válida, o centróide de um chunklet pode estar em uma região vazia. A Figura 6.1(a) ilustra uma situação em que os centróides dos (dois) chunklets estão próximos do centróide da base de dados, em uma região em que não existem objetos. Para evitar esta deficiência, no MCCK os protótipos são inicializados com objetos selecionados aleatoriamente em cada chunklet. Portanto, o número de grupos inicial, que é ajustado durante o processo de agrupamento, é igual ao número de chunklets/classes.

Para simplificar a explicação do MCCK, considere em conjunto com a notação apresentada no Capítulo 4, a representação de restrições de forma matricial. Nesta representação, um conjunto de restrições é representada por uma matriz simétrica $\mathbf{R}_{N \times N}$ cujos elementos são definidos da seguinte forma:

$$
r_{i j}= \begin{cases}1, & \text { se existe uma restrição } \overline{M L} \text { entre } \mathbf{x}_{i} \text { e } \mathbf{x}_{j} \\ -1, & \text { se existe uma restrição } \overline{C L} \text { entre } \mathbf{x}_{i} \text { e } \mathbf{x}_{j} \\ 0, & \text { caso contrário. }\end{cases}
$$

Intuitivamente, se uma restrição é violada quando um objeto é rotulado para um grupo, então o número de grupos (para aquela classe em particular) deve ser insuficiente, e um novo grupo é criado a partir daquele objeto. Esta abordagem também ameniza o efeito outlier, já que as restrições passam a ter um efeito espacial na vizinhança dos objetos envolvidos nas restrições.

\footnotetext{
${ }^{3}$ A definição de chunklets encontra-se na Seção 4.2.

${ }^{4}$ Neste caso, os chunklets correspondem ao conjunto de objetos rotulados de cada classe.
} 
Devido ao processo de criar grupos, é necessário um mapeamento de quais grupos pertencem a quais classes. Inicialmente, esse mapeamento tem uma correspondência de 1-para-1 entre chunklets e grupos. Quando novos grupos são criados, este mapeamento é atualizado - este procedimento é descrito em mais detalhes na sequência.

Objetos que não estão envolvidos em restrições são rotulados para os grupos mais próximos. No entanto, objetos envolvidos em restrições apenas são rotulados para um grupo se nenhuma restrição for violada. Como assume-se que uma classe pode ser representada por mais de um grupo, as restrições não são verificadas considerando apenas os objetos de cada grupo. Ao invés disso, são consideradas as classes conjuntamente com seus respectivos grupos. Por exemplo, suponha que $\mathbf{x}_{n}$ foi rotulado para um grupo $C_{k}$, que $r_{n j}=1$ e que $\mathbf{c}_{m}$ é o protótipo mais próximo de $\mathbf{x}_{j}$. Então, $\mathbf{x}_{j}$ é rotulado para o grupo $C_{m}$ apenas se $C_{m}$ e $C_{k}$ pertencem à mesma classe - isto é verificado pelo mapeamento discutido anteriormente. Se os grupos pertencem a classes diferentes, então um novo grupo é criado, com seu protótipo igual a $\mathbf{x}_{j}$. Para atualizar o mapeamento, inicialmente a classe do chunklet a que $\mathbf{x}_{n}$ pertence é identificada. Então, o novo grupo é adicionado no mapeamento da classe. Pode-se verificar que este processo é sensível a ordem em que os dados são processados. Para amenizar isto, a cada iteração os objetos são processados em ordem aleatória. Depois de todos os objetos serem rotulados para algum grupo, os protótipos (neste caso centróides) são atualizados.

Como a criação dos grupos também é sensível à ordem de apresentação dos objetos, grupos desnecessários podem surgir. Considera-se um par de grupos como desnecessários (redundantes) quando seus protótipos são vizinhos mais próximos mútuos e eles pertencem à mesma classe. Portanto, após a atualização dos protótipos, é verificado se algum par de grupos atende este critério. Se for o caso, estes grupos são unidos. O protótipo do novo grupo é definido como sendo a média dos dois protótipos unidos. Após essa etapa, o mapeamento entre grupos e classes é atualizado.

Os passos principais do MCCK são apresentados no Algoritmo 15. O Passo 11 verifica se a rotulação irá causar alguma violação. Note que embora as restrições $\overline{M L}$ não sejam verificadas diretamente, estas estão sendo implicitamente examinadas ao se verificar as classes/chunklets. Como cada grupo pertence a apenas uma classe, as restrições $\overline{M L}$ não serão violadas, desde que objetos em uma mesma restrição $\overline{M L}$ pertençam a grupos da mesma classe. Os mesmos critérios de parada utilizados no $K$-means são adotados, e.g., um número máximo de iterações ou uma distância mínima entre centróides de iterações consecutivas.

Utilizando o resultado do MCCK, o analista de dados pode sumarizar as classes apropriadamente e analisar suas principais características. Por exemplo, a partir dos grupos que representam cada classe, estatísticas de interesse podem ser derivadas. Adicionalmente, os protótipos dos grupos (i.e., centróides ou objetos representativos) podem ser usados para um melhor entendimento dos dados. Tais informações também podem ser usadas para construir classificadores em uma tarefa subsequente - e.g., como dados de entrada para um classificador $k$-NN (Aha et al., 1991). 


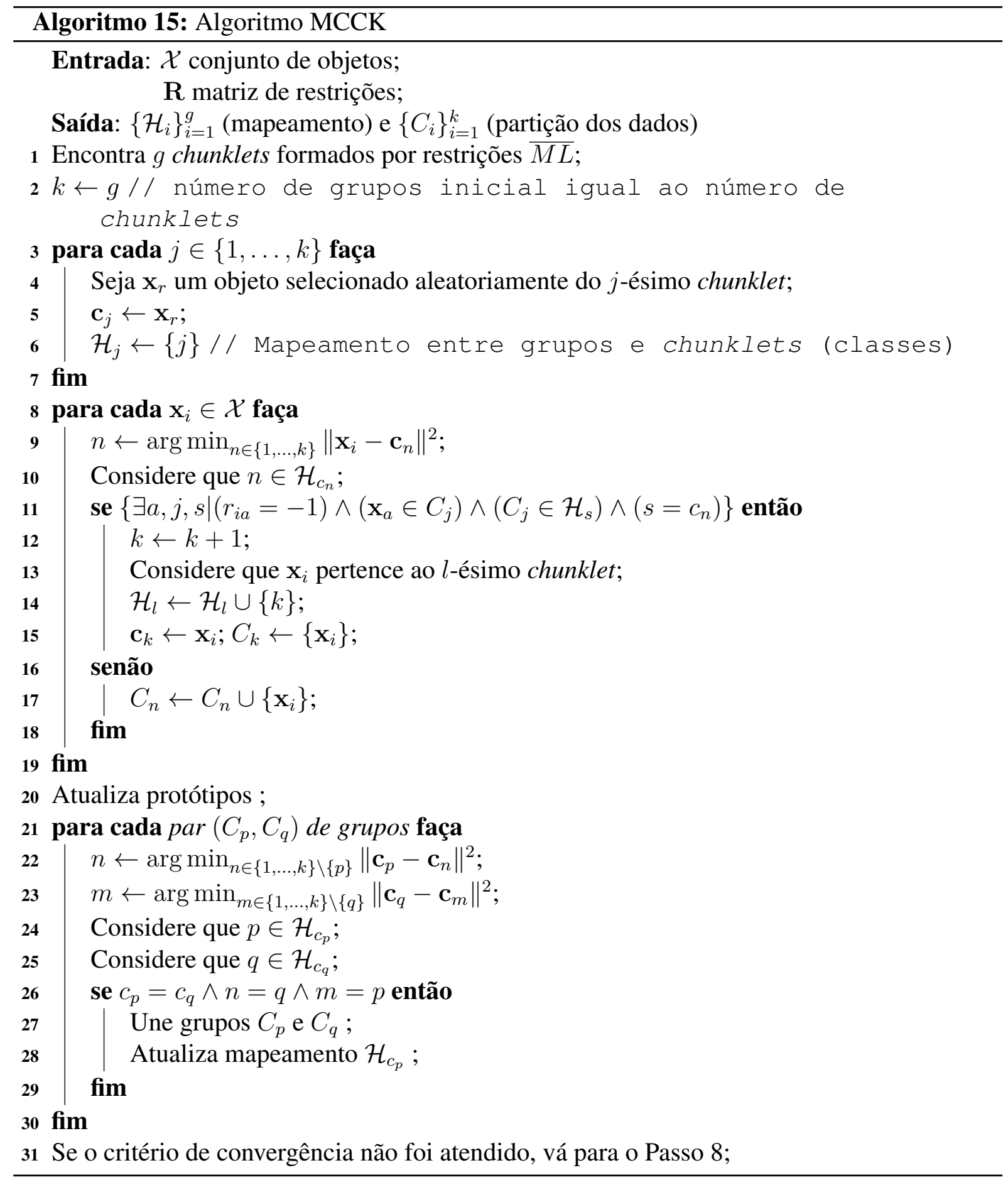




\subsection{Análise Empírica}

Para analisar empiricamente o desempenho do MCCK, decidiu-se comparar seus resultados com aqueles fornecidos pelo COP-K-means (Wagstaff et al., 2001) (como algoritmo baseline) e também com outros dois algoritmos bem-conhecidos na literatura, especificamente: Seeded- $K$ means (Basu et al., 2002) e Constrained-K-means (Basu et al., 2002). Estes algoritmos foram escolhidos por serem bem-conhecidos, assumirem que a supervisão é em forma de rótulos de classes e que, além disso, há um grupo por classe. Dessa forma, pretende-se com esses experimentos apresentar evidências de que assumir um grupo por classe pode ser prejudicial em bases de dados comumente usadas como benchmarks.

Foram realizados experimentos em oito base de dados comumente usadas na literatura. A maioria está disponível no repositório da UCI (Asuncion e Newman, 2007). Adicionalmente, foi utilizada a base de dados 9 Gauss $^{5}$ (Campello et al., 2009). As bases de dados Letters e Pendigits foram utilizadas com apenas as classes I, J, L e 3, 8 e 9 - conforme feito na Seção 5.5 e em Bilenko et al. (2004). Finalmente, foi utilizada também a base de dados Ovarian Cancer (Petricoin III et al., 2002). As principais características das bases de dados utilizadas são sumarizadas na Tabela 6.1.

Tabela 6.1: Bases de dados usadas nos experimentos.

\begin{tabular}{cccc}
\hline Base de dados & $\mathbf{N}$ & $\mathbf{M}$ & \# Classes \\
\hline 9Gauss & 900 & 2 & 9 \\
Breast Cancer & 683 & 9 & 2 \\
Ionosphere & 351 & 34 & 2 \\
Iris & 150 & 4 & 3 \\
Letters & 2.263 & 16 & 3 \\
Ovarian Cancer & 253 & 15.154 & 2 \\
Pendigits & 3.165 & 16 & 3 \\
Wine & 178 & 13 & 3 \\
\hline
\end{tabular}

Conforme mencionado na Seção 6.1, considera-se o cenário em que restrições são derivadas de objetos rotulados. Para a geração de restrições, foram considerados casos em que o número de objetos rotulados por classe pertence ao conjunto $\{3,4,5,6,7,10,15,20,25,30\}$. De cada conjunto de objetos rotulados, todas as restrições $\overline{M L}$ e $\overline{C L}$ são deduzidas. Os objetos usados para derivar as restrições foram selecionados aleatoriamente. Devido à sensibilidade na inicialização dos centróides e na ordem de processamento dos objetos, o algoritmo MCCK foi executado cinco vezes. A melhor partição, de acordo com o critério da Silhueta Simplificada (Hruschka et al., 2006) ${ }^{6}$, foi selecionada. Todo o processo foi repetido 100 vezes para se obter melhores estimativas das estatísticas de interesse.

As análises foram divididas em duas etapas. Na primeira etapa foram comparadas as partições obtidas por cada algoritmo, enquanto que, na segunda etapa, o impacto da quantidade de supervisão fornecida no erro de classificação é analisado.

\footnotetext{
${ }^{5}$ Descrita na Seção 3.4 .

${ }^{6}$ Descrita na Seção 2.4.1.1.
} 
Tabela 6.2: Número médio de grupos por classe estimados pelo MCCK (considerando todas as quantidades de objetos rotulados).

\begin{tabular}{|c|c|c|c|c|c|c|c|c|c|c|c|}
\hline \multirow{3}{*}{$\begin{array}{c}\text { Base de dados } \\
\text { Classe } \\
\text { \# Grupos }\end{array}$} & \multicolumn{3}{|c|}{ Letters } & \multicolumn{3}{|c|}{ Wine } & \multicolumn{2}{|c|}{ Ovarian Cancer } & & \multicolumn{2}{|c|}{ Ionosphere } \\
\hline & $\mathbf{I}$ & $\mathbf{J}$ & $\mathbf{L}$ & C1 & $\mathrm{C2}$ & C3 & Cancer & Normal & & Good & Bad \\
\hline & 7 & 9 & 5 & 6 & 10 & 11 & 6 & 7 & & 6 & 9 \\
\hline Base de dados & & Iris & & & Brea & Cancer & & & digi & & \\
\hline Classe & Setosa & Versicolor & Virginica & & Benign & Malign & & $\# \mathbf{3}$ & \#8 & \#9 & \\
\hline \# Grupos & 1 & 4 & 4 & & 2 & 4 & & 6 & 4 & 11 & \\
\hline Base de dados & & & & & 9Ga & & & & & & \\
\hline Classe & C1 & $\mathrm{C2}$ & C3 & $\mathrm{C4}$ & C5 & C6 & C7 & C8 & C9 & & \\
\hline \# Grupos & 5 & 6 & 5 & 6 & 7 & 6 & 5 & 6 & 5 & & \\
\hline
\end{tabular}

\subsubsection{Comparando as Partições dos Dados}

A qualidade das partições obtidas foi avaliada utilizando o Adjusted Rand Index (ARI) (Hubert e Arabie, 1985; Vinh et al., 2010), comparando as partições de referência fornecidas pelas classes com as obtidas por cada algoritmo. Para o MCCK, a partição resultante é obtida rerotulando cada rótulo de grupo pelo rótulo de sua classe.

As Figuras 6.2(a)-6.2(h) apresentam a média de ARI obtida por cada algoritmo. Inicialmente, considere os resultados obtidos para a base de dados 9Gauss (Figura 6.2(a)). Como esta é uma base de dados artificial, o número de grupos correto é conhecido, e que neste caso corresponde ao número de classes. Portanto, este é um exemplo de uma base de dados para a qual a premissa de um grupo por classe é válida. É possível verificar que os algoritmos da literatura podem obter partições com valores altos de ARI (os erros são devido à sobreposição entre os grupos), enquanto que o MCCK obtém os piores resultados. Isto é esperado porque o algoritmo pode se confundir por causa de restrições envolvendo alguns poucos objetos nas regiões de sobreposição. Resultados similares foram obtidos nas bases de dados Breast Cancer e Wine Figuras 6.2(b) e 6.2(h). No entanto, MCCK obtém resultados sensivelmente melhores que os demais algoritmos nas bases de dados Ionosphere, Iris, Letters, Ovarian Cancer e Pendigits. Estes resultados sugerem que, para estas bases de dados, a premissa de um grupo por classe não se sustenta. De fato, a Tabela 6.2 mostra que, na maioria dos casos, MCCK encontrou mais de dois grupos por classe.

\subsubsection{Avaliando o Erro de Classificação}

Nesta seção, foi avaliado o erro de classificação obtido considerando as partições encontradas pelos algoritmos, como se estas fossem resultados obtidos por classificadores. Para encontrar o mapeamento ótimo entre grupos e classes, o algoritmo Húngaro é utilizado (Kuhn, 1955). Apesar de classificação semissupervisionada não ser o foco principal deste trabalho, esta medida é útil por permitir mensurar o impacto da supervisão no processo de agrupamento. Especificamente, tendo como foco a análise exploratória de dados, para qual sumarização de dados é de grande importância, a relação do erro de classificação em função da quantidade de objetos rotulados pode ser importante. Além disso, analisando o impacto no uso de restri- 


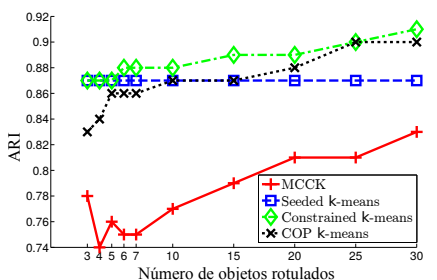

(a) 9Gauss

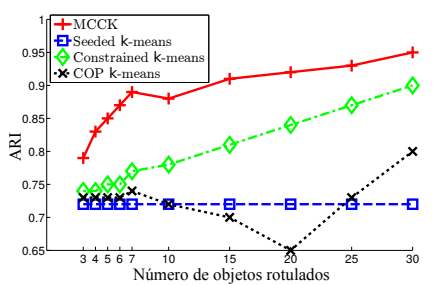

(d) Iris

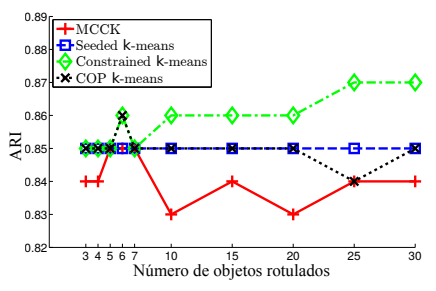

(b) Breast Cancer

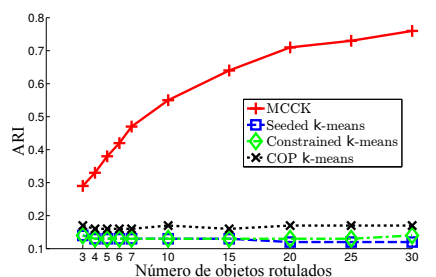

(e) Letters

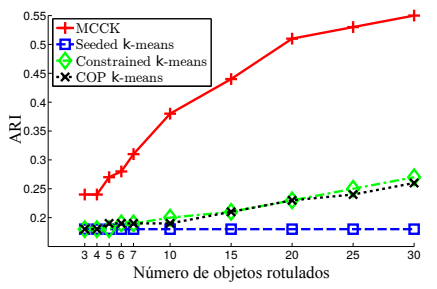

(c) Ionosphere

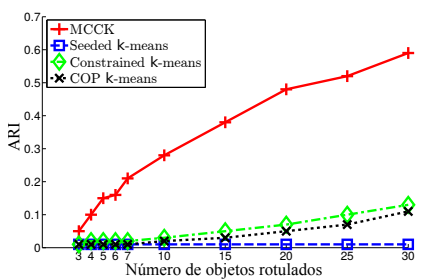

(f) Ovarian Cancer

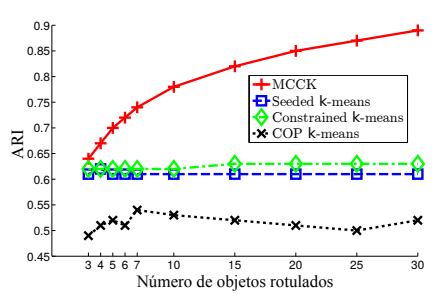

(g) Pendigits

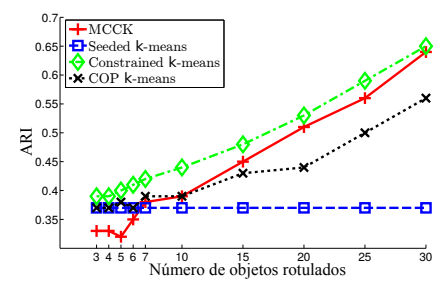

(h) Wine

Figura 6.2: Valores médios de ARI obtidos para cada quantidade de objetos rotulados por classe.

ções obtidas a partir de poucos objetos rotulados, pode-se observar que a acurácia do MCCK é melhor do que os demais algoritmos analisados.

Os resultados da taxa de erro médio obtidos usando 3, 7 e 30 objetos rotulados por classe para gerar as restrições são apresentados na Tabela 6.3. Comparando as taxas de erro obtidas com três e sete objetos rotulados, pode-se observar que um pequeno aumento no número de objetos rotulados permite ao MCCK apresentar uma maior redução no erro - i.e., na média $4 \%$ contra $1 \%$ do Constrained- $K$-means e COP- $K$-means, enquanto para o Seeded- $K$-means o erro aumentou em cerca de $0,1 \%$. Isto indica que, mesmo com uma pequena quantidade de supervisão, MCCK a incorpora satisfatoriamente no processo de agrupamento. Especificamente, para as bases de dados Letters e Ovarian Cancer, melhoras de mais de $11 \%$ foram obtidas. Além disso, ao considerar a redução de erro obtida usando 30 e 3 objetos rotulados, existem diferenças significativas de acurácia entre os algoritmos estudados. Neste caso, o MCCK obteve uma redução de erro de $13 \%$ (na média), contra uma redução de $6 \%$ do Constrained- $K$-means e COP-K-means e um aumento de erro de $0,1 \%$ do Seeded-K-means.

\subsection{Considerações Finais}

Neste capítulo, foi apresentado um novo algoritmo de ADR, denominado Multiple Clusters per Class K-means (MCCK). Diferentemente de outros algoritmos de ADR, MCCK é de uso 
Tabela 6.3: Taxas de erro médias (\%) para diferentes quantidades de objetos rotulados por classe (\# OR).

\begin{tabular}{|c|c|c|c|c|c|c|c|c|c|c|c|c|}
\hline \multirow{2}{*}{$\begin{array}{c}\text { Algoritmo } \\
\text { \#OR }\end{array}$} & \multicolumn{3}{|c|}{ MCCK } & \multicolumn{3}{|c|}{ Seeded $K$-means } & \multicolumn{3}{|c|}{ Constrained $K$-means } & \multicolumn{3}{|c|}{ COP K-means } \\
\hline & 3 & 7 & 30 & 3 & 7 & 30 & 3 & 7 & 30 & 3 & 7 & 30 \\
\hline 9Gauss & 10.86 & 12.70 & 7.85 & 6.19 & 6.18 & 6.13 & 5.98 & 5.69 & 4.27 & 9.86 & 6.67 & 4.83 \\
\hline Letters & 34.48 & 22.58 & 8.75 & 50.55 & 51.53 & 51.67 & 50.43 & 51.10 & 49.18 & 48.04 & 48.33 & 47.30 \\
\hline Wine & 33.79 & 29.36 & 13.84 & 29.78 & 29.78 & 29.78 & 28.53 & 26.19 & 13.62 & 30.74 & 29.81 & 19.45 \\
\hline Ovarian Cancer & 39.80 & 28.13 & 11.47 & 43.91 & 43.89 & 43.57 & 43.72 & 43.00 & 32.31 & 44.36 & 44.28 & 34.12 \\
\hline Ionosphere & 26.25 & 22.66 & 13.02 & 28.83 & 28.80 & 28.83 & 28.63 & 28.29 & 24.16 & 28.77 & 28.18 & 24.70 \\
\hline Iris & 8.38 & 4.11 & 1.68 & 11.15 & 11.19 & 11.30 & 10.33 & 9.01 & 3.63 & 10.77 & 10.54 & 8.81 \\
\hline Breast Cancer & 4.09 & 4.08 & 4.34 & 3.90 & 3.88 & 3.87 & 3.85 & 3.78 & 3.37 & 3.90 & 3.88 & 3.89 \\
\hline Pendigits & 14.68 & 9.81 & 3.93 & 14.62 & 14.25 & 14.25 & 14.49 & 14.03 & 13.55 & 30.24 & 23.98 & 26.13 \\
\hline
\end{tabular}

mais amigável, uma vez que não necessita que o usuário forneça valores para parâmetros que são difíceis de se ajustar na prática. De fato, o MCCK necessita apenas do conjunto de dados e de um conjunto de restrições ML e CL derivadas de objetos rotulados, sob a premissa de que pelo menos dois objetos por classe estão rotulados.

Resultados experimentais evidenciam que para algumas bases de dados, que costumam ser usadas como benchmark, a premissa de um grupo por classe não é válida. Em especial, o MCCK foi capaz de apresentar resultados melhores que algoritmos tradicionais de agrupamento semissupervisionado, até mesmo sendo capaz de reduzir o erro de classificação mesmo quando a quantidade de objetos rotulados fornecidos era pequena.

$\mathrm{O}$ algoritmo desenvolvido, bem como as análises experimentais reportadas nesta tese, foram publicados em Sestaro et al. (2012). Além disso, o MCCK também foi utilizado em conjunto com uma abordagem de construção de árvores de decisão de forma bottom-up. Neste trabalho, o MCCK realizou o papel de decompor um problema de classificação em subproblemas que fossem fáceis de serem resolvidos por uma Máquina de Vetor de Suporte Transdutiva com kernel linear. O resultado dessa frente de pesquisa foi publicado em Covões et al. (2013b) ${ }^{7}$.

No próximo capítulo, é apresentado um algoritmo que estende as ideias do MCCK para o modelo de matrizes de covariâncias irrestritas. Para atacar este problema, faz-se uso de um Algoritmo Evolutivo (AE).

\footnotetext{
${ }^{7}$ Trabalho premiado como melhor artigo completo do Simpósio Brasileiro de Banco de Dados (SBBD) de 2013
} 
CAPÍTULO

\section{Algoritmo Evolutivo para Modelos de Mistura de Gaussianas que Utiliza Restrições}

\subsection{Considerações Inciais}

Este capítulo aborda o algoritmo Feasible-Infeasible Evolutionary Create \& Eliminate Algorithm for Expectation Maximization (FIECE-EM). Este algoritmo pode ser visto como uma generalização do algoritmo MCCK apresentado no Capítulo 6. Conforme discutido no Capítulo 2, Modelos de Mistura de Gaussianas (GMMs) com matrizes de covariância irrestrita são capazes de aproximar funções de densidade contínuas com acurácia arbitrária. Considerando aplicações de agrupamento de dados com restrições, é possível que um GMM modele grupos com formas complexas, i.e., que não tenham formato (hiper)elipsoidal.

A incorporação de restrições no modelo generalizado não é um processo trivial. Isso acontece devido à introdução da dependência nos vetores indicadores $\left(\mathcal{Z}=\{\mathbf{z}\}_{n=1}^{N}\right)$. Essa dependência torna o problema mais difícil de ser modelado, já que a probabilidade de um objeto ser de um grupo depende de todos os objetos que possuem alguma restrição com ele. Por isso não é possível obter solução fechada para todos os parâmetros do GMM, e torna-se necessário que aproximações sejam feitas (Basu et al., 2008; Shental et al., 2003; Zhao e Miller, 2005; Raghuram et al., 2014).

No FIECE-EM uma abordagem diferente é adotada. Ao invés de utilizar um modelo gerador complexo que incorpore as restrições, considera-se o modelo sem restrições, porém diferenciando modelos que não violam nenhuma restrição. Para realizar essa busca, toma-se por base um algoritmo bem-conhecido na literatura de AEs denominado Feasible-Infeasible Two- 
Population (FI2POP) (Kimbrough et al., 2002, 2003), que é descrito brevemente na próxima seção. Dada a sua importância prática, a literatura sobre AEs para problemas de otimização com restrições é extensa. Caso o leitor esteja interessado em uma revisão profunda sobre o assunto, sugere-se os trabalhos de Michalewicz (1995); Coello (2002); Eiben e Smith (2003).

\subsection{Feasible-Infeasible Two-Population}

O algoritmo FI2POP foi proposto por Kimbrough et al. (2002), e proporciona bons resultados quando comparado com outras abordagens evolutivas para problemas de otimização com restrições (Kimbrough et al., 2008). A ideia principal do algoritmo FI2POP consiste em evoluir duas populações de soluções em paralelo. Na primeira, denominada feasible, todos os indivíduos são soluções factíveis para o problema, i.e., atendem a todas as restrições. Enquanto que na outra população, chamada de infeasible, tem-se apenas soluções que violam ao menos uma restrição. Cada população é evoluída por um AE tradicional ${ }^{1}$, tendo como principal diferença a função objetivo utilizada. Na população feasible a função objetivo é aquela que seria usada sem levar em consideração as restrições. A qualidade da população infeasible, por sua vez, é mensurada de acordo com as restrições sendo violadas pela solução, e.g., o número de restrições violadas. Portanto, enquanto uma população é direcionada em busca da melhor solução possível para o problema, a outra busca por soluções factíveis.

$\mathrm{O}$ algoritmo FI2POP realiza a troca de indivíduos entre suas populações. Mais especificamente, em cada geração é possível que novas soluções factíveis (não-factíveis) sejam geradas pela população infeasible (feasible), durante o processo de mutação. O fato de soluções nãofactíveis serem evoluídas para soluções factíveis permite ao algoritmo explorar as regiões de fronteira. Diferentemente de outros algoritmos, como o bem-conhecido GENOCOP (Michalewicz e Nazhiyath, 1995; Michalewicz, 1995), não há necessidade de que os operadores se limitem a gerar apenas soluções factíveis. Além das duas populações, são mantidos dois pools (feasible e infeasible), nos quais novos indivíduos são inseridos de acordo com sua factibilidade. Indivíduos nestes pools são considerados para a etapa de seleção de sobreviventes para a geração subsequente. Essa troca de indivíduos entre as duas populações auxilia a manter a diversidade, evitando convergência prematura, e aumenta a capacidade de exploração do espaço de soluções.

Uma vantagem do FI2POP em relação a outros AEs para problemas de otimização com restrições é não depender de que soluções factíveis sejam conhecidas/geradas para o início da execução. Tanto a população feasible quanto a infeasible podem ser vazias no início, e serem populadas conforme novos indivíduos são evoluídos. O fato da avaliação da factibilidade das restrições ser realizada de forma desacoplada da avaliação da adequabilidade dos indivíduos simplifica o desenvolvimento de algoritmos específicos para cada domínio de aplicação, uma

\footnotetext{
${ }^{1}$ Os componentes, e.g., operador de seleção, mutação, etc, podem ser definidos de acordo com a aplicação e com os recursos computacionais disponíveis.
} 
vez que não é necessário definir critérios/parâmetros de tradeoff entre qualidade e factibilidade de restrições.

\subsection{Feasible-Infeasible Evolutionary Create \& Eliminate Algo- rithm for Expectation Maximization}

O algoritmo FIECE-EM pode ser considerado como uma instância do FI2POP voltada para o problema de ADR com GMMs. Inicialmente, é importante definir as premissas do FIECE-EM. Assume-se que as restrições ML e CL são referentes a classes e não a grupos, i.e., são restrições $\overline{M L}$ e $\overline{C L}$. Além disso, assume-se também uma bijeção entre classes e chunklets e que todo objeto envolvido em uma restrição $\overline{C L}$ também está envolvido em pelo menos uma restrição $\overline{M L}$. Portanto, as premissas são as mesmas que as do algoritmo MCCK (Capítulo $6)$.

A codificação do indivíduo é similar à utilizada pelo ESM-EM, com a adição de uma matriz binária $\mathbf{M}_{g \times K}$ que representa o mapeamento dos $g$ chunklets para os $K$ grupos representados no GMM. Especificamente, cada elemento dessa matriz, $m_{i j}$, é igual a 1 se o grupo $j$ representa a classe/chunklet $i$, e zero caso contrário, com a restrição que $\sum_{i=1}^{g} m_{i j}=1, \forall j \in\{1, \ldots, K\}$. Os principais passos do algoritmo FIECE-EM são apresentados no Algoritmo 16. Detalhes específicos de cada passo do algoritmo são descritos nas subseções a seguir.

\subsubsection{Inicialização}

O número de grupos iniciais utiliza a mesma estratégia adotada no ESM-EM (Capítulo 3), i.e., valores linearmente espaçados entre o número mínimo de grupos, $K_{\min }$ (que é igual ao número de chunklets) e o número máximo de grupos $\left(K_{\max }\right)$. Para gerar soluções viáveis, se sorteia um chunklet para cada grupo aleatoriamente (garantindo que todas as classes estão presentes). Para cada grupo, um dos objetos do chunklet da classe é selecionado aleatoriamente e usado como semente para o $K$-means. Caso a solução gerada seja inviável, o próximo número de grupos $\left(K_{\min }+1\right)$ é empregado. Isso é feito até que se consiga gerar a quantidade de indivíduos desejada ou se atinja um número máximo de tentativas. Nesse caso, o algoritmo prossegue mesmo que não existam indivíduos viáveis gerados. Especificamente, a população feasible pode ser vazia tanto no início do algoritmo quanto nas gerações seguintes. A geração de indivíduos inviáveis ocorre de forma similar. No entanto, os objetos usados como centróides iniciais para o $K$-means são escolhidos aleatoriamente, i.e., ignorando as restrições. O mapeamento entre grupos e chunklets também é inicializado aleatoriamente. 


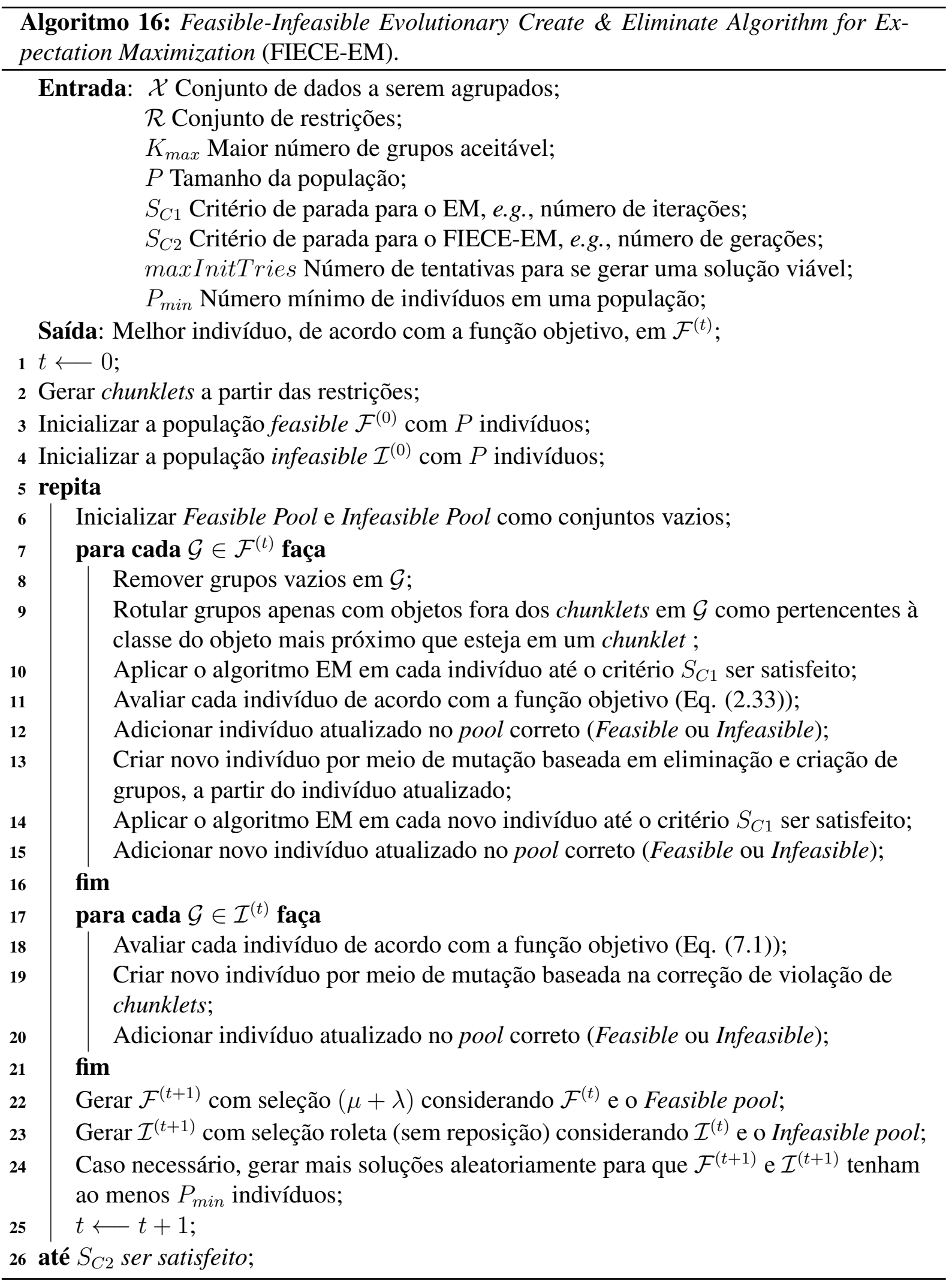




\subsubsection{Função Objetivo}

Para a população feasible avalia-se apenas a qualidade do modelo em relação aos dados. Portanto, o FIECE-EM utiliza a mesma função objetivo utilizada no ESM-EM — Equação (2.33). No caso da população infeasible a função objetivo deve capturar informação da factibilidade da solução. Para isto, utiliza-se do mapeamento entre grupos e chunklets (classes). Especificamente, considerando $\left\{\Lambda_{i}\right\}_{i=1}^{g}$ o conjunto de chunklets e a matriz de mapeamento $\mathbf{M}_{g \times K}$, a função objetivo é definida como:

$$
\begin{gathered}
J_{\text {infeasible }}(\mathcal{G})=\sum_{i=1}^{g} \sum_{\mathbf{x}_{n} \in \Lambda_{i}} c V i o\left(n, \underset{c \in\{1, \ldots, K\}}{\arg \max } \gamma\left(z_{n c}\right), i, \mathbf{M}\right), \\
c V i o(n, c, i, \mathbf{M})= \begin{cases}0 & \text { se } m_{i c}=1, \\
1-\max \left(\left\{\gamma\left(z_{n k}\right) \mid \forall k, m_{i k}=1\right\}\right) & \text { caso contrário, }\end{cases}
\end{gathered}
$$

dessa forma as restrições são ditas "violadas" se o grupo ao qual o objeto pertence não está mapeado para o chunklet do objeto. Cada violação recebe um valor de penalidade, que considera o grupo em que o objeto deveria estar. Considerando o conjunto de grupos mapeados para o chunklet do objeto, presume-se que o objeto deveria estar no grupo deste conjunto com maior responsabilidade sobre este objeto. Portanto, o valor de penalização é inversamente proporcional à esta responsabilidade. Ambas as funções podem ser vistas como problemas de minimização. Cada população feasible e infeasible é avaliada apenas pela função objetivo apropriada.

\subsubsection{Operadores de Mutação}

As mutações em ambas as populações são realizadas por dois operadores: eliminação e criação de grupos. Os procedimentos que realizam a eliminação e criação de grupos são apresentados nos Algoritmos 17 e 18. A eliminação de um grupo consiste basicamente na remoção dos parâmetros do respectivo componente e re-normalização dos demais parâmetros do GMM. Para a criação de grupos é utilizada uma heurística, baseada no método de inicialização de componentes descrito por Figueiredo e Jain (2002), que força a nova componente a ter um domínio espacial na vizinhança da sua média, independente da estrutura dos componentes existentes anteriormente. Para isso, inicializa-se os novos componentes com a matriz de covariância igual a um décimo de $\mathbf{V}$, sendo $\mathbf{V}$ uma matriz diagonal com elementos iguais à variância de cada atributo, i.e., $v_{i i}=\sigma_{i}^{2}, \forall i \in\{1, \ldots, M\}$. A classe do novo grupo criado é definida como a classe do objeto, presente em um chunklet, mais próximo. Tal operador pode ser considerado menos principiado do que os operadores de divisão de componentes descritos na Seção 2.3, justamente por fazer menos uso da estrutura do GMM para a definição dos valores do novo componente a ser inserido. No entanto, os experimentos apresentados em Covões e Hruschka (2013) sugerem que este tipo de operador de criação de grupos não afeta significativamente a capacidade de exploração do espaço, e é mais eficiente computacionalmente. 

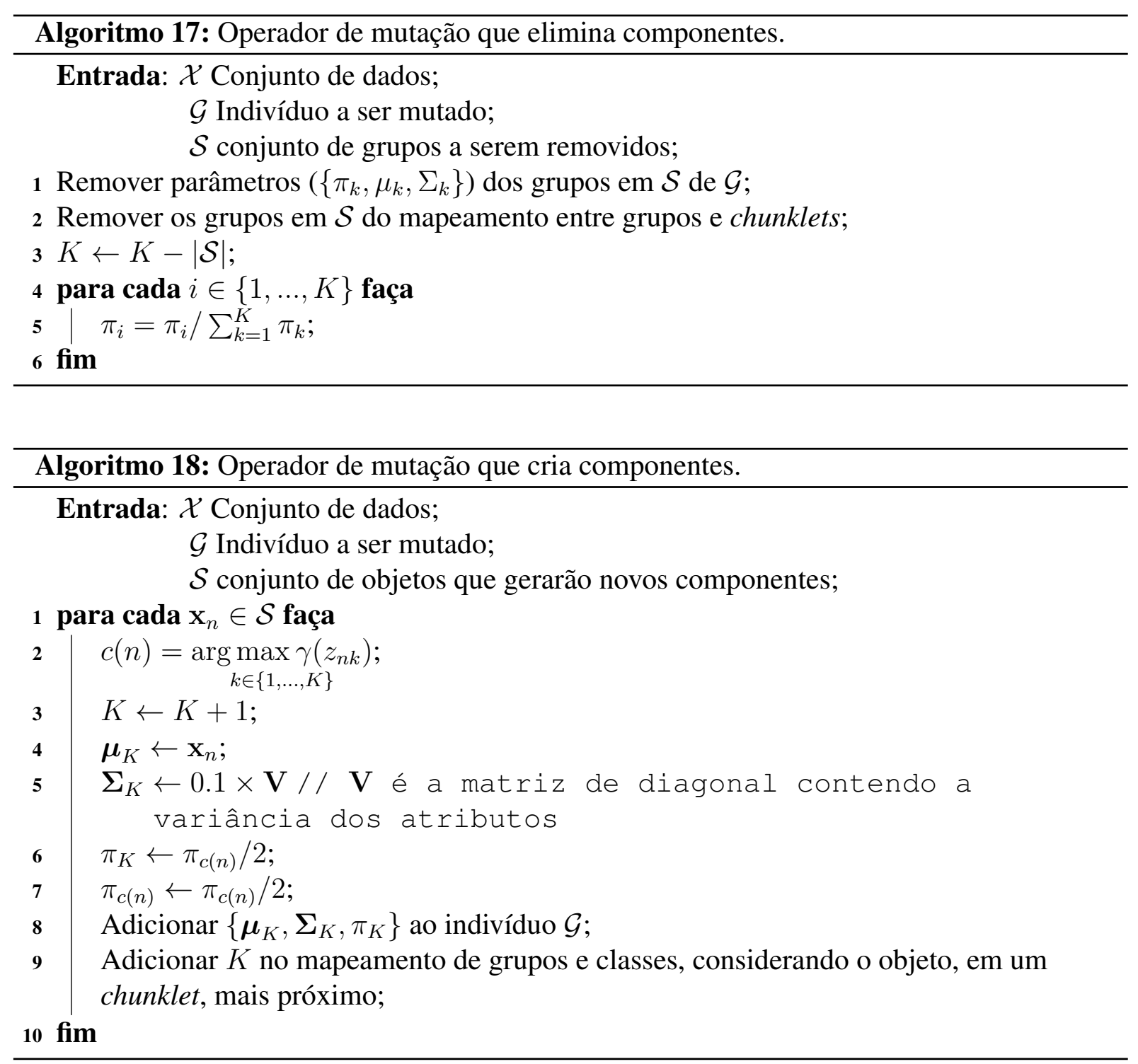

No caso da população feasible, a probabilidade do operador de eliminação ser aplicada é igual a:

$$
p_{e}=\frac{K-K_{\min }}{K_{\max }-K_{\min }}
$$

sendo $K$ o número de grupos da solução, $K_{\min }$ e $K_{\max }$ e menor e maior número de grupos permitidos, respectivamente. Consequentemente, a probabilidade do operador de criação de grupos ser aplicado é igual a 1- $p_{e}$. Para definir qual grupo deve ser eliminado é utilizado o Partial Log-Likelihood (PLL) (Equação 3.1) em conjunto com a seleção por roleta baseada em ranking. São selecionados até $K-K_{\text {min }}$ grupos para remoção, com a ressalva de que se o grupo for o único de determinada classe/chunklet ele não é removido. No caso de aplicação do operador de criação, são selecionados até $K_{\max }-K$ objetos de acordo com a entropia de suas probabilidades a posteriori $\left(\gamma\left(z_{n k}\right), n \in\{1, \ldots, N\}\right.$ e $\left.k \in\{1, \ldots, K\}\right)$, utilizando o método roleta e normalização por ranking (Bäck et al., 2000a).

A mutação na população infeasible ocorre de forma similar. No entanto, as violações de 
restrições guiam quais grupos devem ser removidos/criados. Especificamente, para cada objeto em um chunklet é verificado se o grupo a qual ele pertence ${ }^{2}$ está mapeado para a mesma classe/chunklet do objeto. Como a população é formada apenas por soluções infeasible, ao menos um objeto está em um grupo de outro chunklet. Utilizando o custo das violações das restrições, derivado da Equação (7.2), são selecionados, utilizando seleção por roleta, até $K_{\max }-K$ objetos para serem usados como sementes para novos grupos. Caso $K=K_{\max }$ são selecionados até $K_{\max }-K_{\min }$ grupos para serem removidos. É importante notar que, após selecionados, os grupos apenas são removidos se eles não são os únicos a representar um chunklet, evitando dessa forma que uma classe não seja representada pela solução.

\subsubsection{Seleção de Sobreviventes}

A seleção realizada em cada população é diferente. Na população de soluções factíveis, é utilizada uma seleção $(\mu+\lambda)$, que é mais conservadora e mantém a pressão seletiva alta. A vantagem dessa escolha é auxiliar o acúmulo de refinamentos do EM que, sabidamente, demora diversas iterações para convergir.

Na população de soluções inviáveis, é utilizada uma seleção por roleta (sem reposição). Com menor pressão seletiva, essa alternativa auxilia a manter a diversidade de soluções nesta população.

\subsubsection{Cuidados Adicionais}

É possível que com a criação/eliminação de grupos uma solução passe a ter grupos vazios, i.e., componentes que não são os maiores responsáveis por nenhum objeto. Por essa razão, a cada geração os indivíduos da população feasible passam por uma etapa de remoção de grupos vazios. Além disso, é possível que, algumas iterações após ter sido criado, um grupo não possua nenhum objeto em chunklets, e por isso o mapeamento para a classe pode ter perdido o sentido. Neste caso, a classe é mapeada para o objeto mais próximo que está em um chunklet. Finalmente, caso o usuário deseje evitar que alguma das populações fiquem vazias de uma geração para outra, pode-se utilizar o parâmetro $P_{\min }$ para que ao fim das gerações seja possível inicializar novos indivíduos factíveis ou não-factíveis.

\subsection{Exemplo Pedagógico}

Para auxiliar o entendimento do funcionamento do algoritmo FIECE-EM, alguns passos da execução do algoritmo em uma base de dados sintética são apresentados. Considere os dados apresentados na Figura 7.1. Embora os dados estejam dispostos em 3 grupos, os rótulos de classe indicam que as 3 classes não correspondem a estes grupos. Este problema pode ser

\footnotetext{
${ }^{2}$ Devido ao particionamento probabilístico realizado pelo GMM, o grupo a que o objeto pertence refere-se ao componente com maior responsabilidade $\left(\gamma\left(z_{n k}\right)\right)$ pelo objeto.
} 


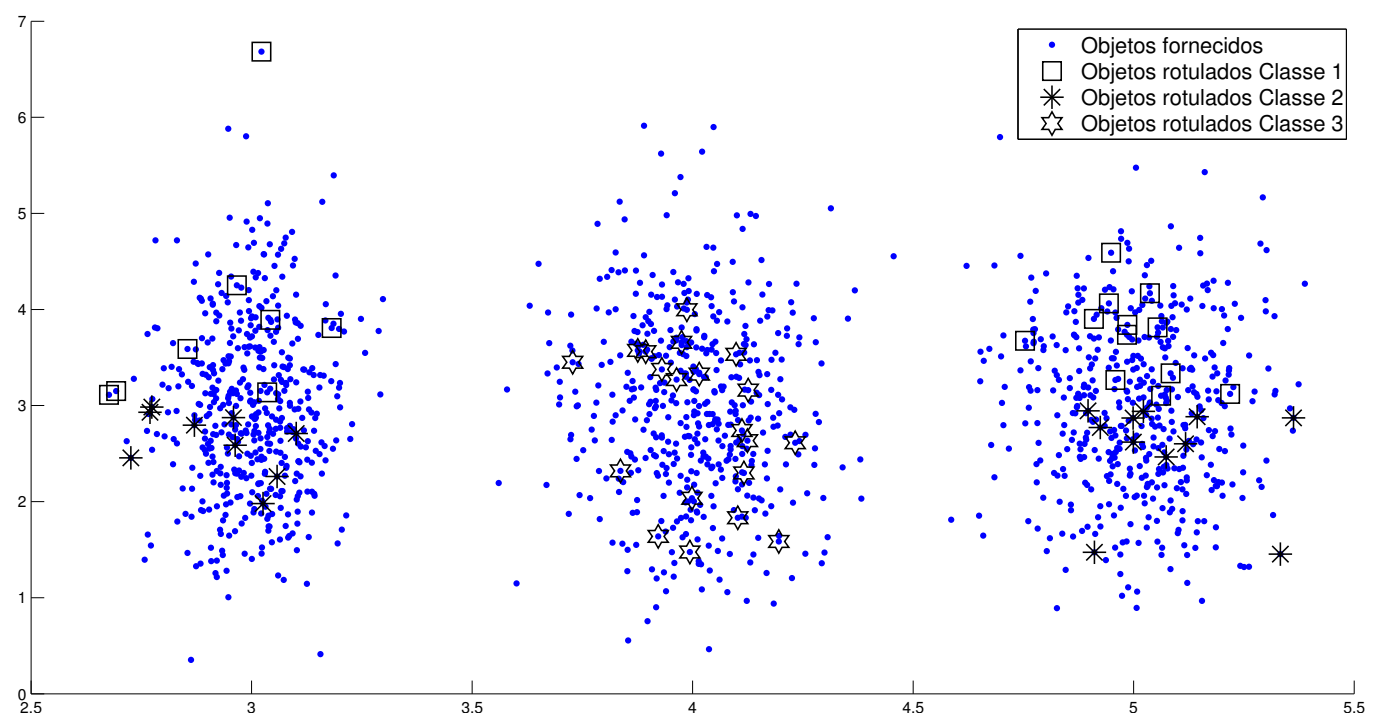

Figura 7.1: Base de dados sintética.

considerado difícil uma vez que a região de fronteira entre os objetos da classe 1 e 2 é densa e com indícios de sobreposição. Obtendo-se as restrições a partir dos objetos rotulados, pode-se iniciar a execução algoritmo.

Gerar a população infeasible inicial não é um problema. No entanto, na $1^{\text {a }}$ geração nenhum indivíduo feasible foi gerado. Isto é esperado, visto que a inicialização dos indivíduos feasible utiliza como sementes os objetos rotulados e eles se encontram muito próximos uns dos outros, o que sugere que estão próximos à fronteira entre as duas classes. Por tal razão, este é um exemplo em que inicializar aleatoriamente soluções válidas é mais difícil do que obtê-las a partir de soluções inválidas. Esta capacidade é justamente um dos pontos fortes do algoritmo FI2POP e, consequentemente, do FIECE-EM. Na Figura 7.2 é apresentado um dos GMM gerados aleatoriamente na $1^{a}$ geração. É fácil observar que o número de grupos é maior que o ideal. No entanto, as principais regiões de interesse estão razoavelmente bem modeladas, sendo relativamente fácil imaginar uma série de operações (remoção e criação de grupos) capazes de tornar tal modelo feasible de acordo com as restrições. Por exemplo, a remoção dos quatro componentes que possuem grande variância em apenas uma das direções (elipses alongadas, sendo três na região de coordenadas $y=1$ e um próximo ao ponto de coordenada $x=5$ e $y=6$ ) e a troca de classe de dois componentes que não possuem objetos rotulados e não estão mapeados para a classe dos objetos rotulados mais próximos (componente próximo a coordenada $x=4$ e $y=5$ e o componente com média na coordenada $x=5,3$ e $y=1,45)$. Com tais alterações o GMM resultante teria tanto um bom ajuste aos dados como às restrições.

Embora nenhum indivíduo feasible tenha sido criado, o algoritmo prossegue as gerações realizando operações nos indivíduos infeasible a fim de diminuir as violações de restrições neles observadas. $\mathrm{O} 1^{\mathrm{o}}$ indivíduo feasible é gerado apenas na $3^{\mathrm{a}}$ geração a partir do indivíduo infeasible da Figura 7.3. Assim como o indivíduo apresentado na Figura 7.2, este indivíduo apresenta um número maior de grupos que o ideal e com grupos da mesma classe muito próximos uns 


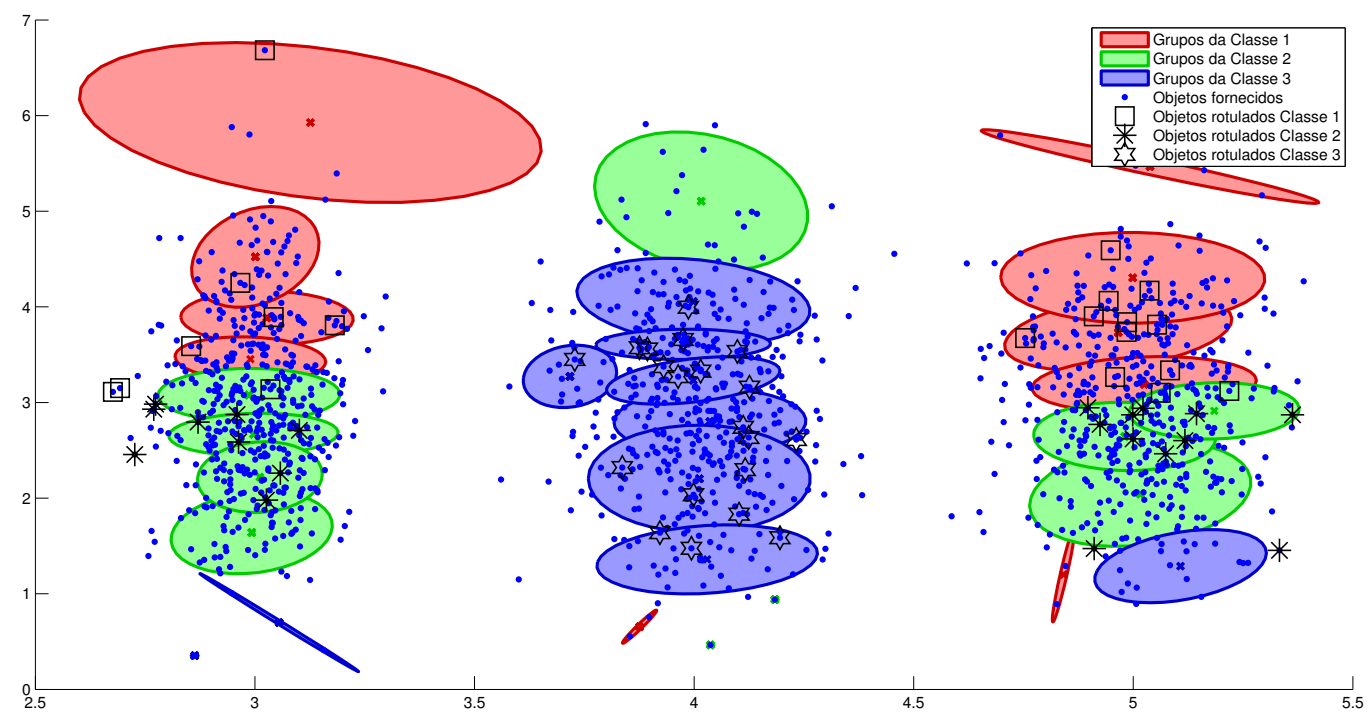

Figura 7.2: Indivíduo infeasible da $1^{\mathrm{a}}$ geração.

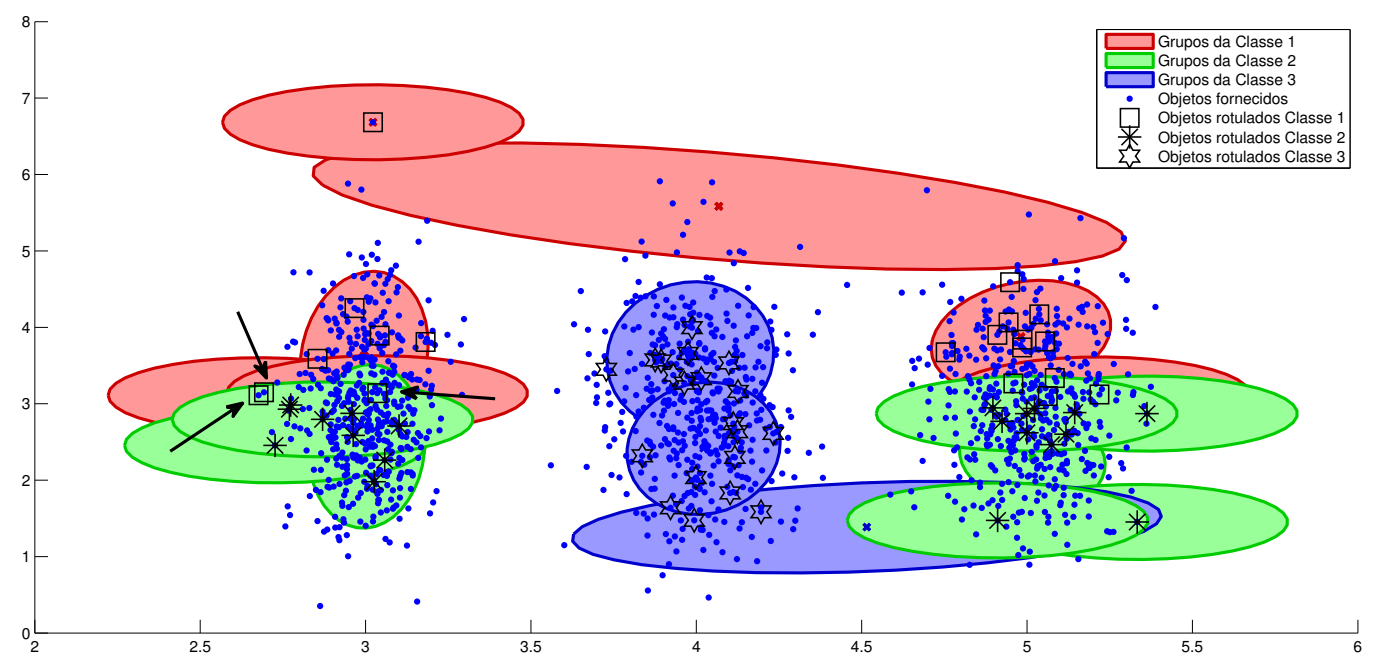

Figura 7.3: Indivíduo infeasible que origina o primeiro indivíduo feasible. Objetos que estão violando restrições são indicados pelas setas.

dos outros. O processo de transformar este indivíduo em um indivíduo feasible iniciou com a criação de 3 grupos, um sob cada objeto que estava violando restrições. Estes objetos são indicados pelas setas na Figura 7.3. O modelo resultante da criação destes grupos é apresentado na Figura 7.4.

Como uma nova solução feasible foi gerada, é verificado se existem grupos vazios (componentes que não possuem a maior responsabilidade para nenhum objeto). Caso existam, como no caso, estes são removidos. Especificamente, três grupos foram removidos, todos na região do ponto $x=2,7$ e $y=3$. Finalmente, após estes ajustes o GMM feasible resultante é apresentado na Figura 7.5.

$\mathrm{Na} 4^{\mathrm{a}}$ geração após a etapa de refinamento, com apenas um único indivíduo feasible disponível, pela primeira vez durante a execução do algoritmo o procedimento de mutação de 


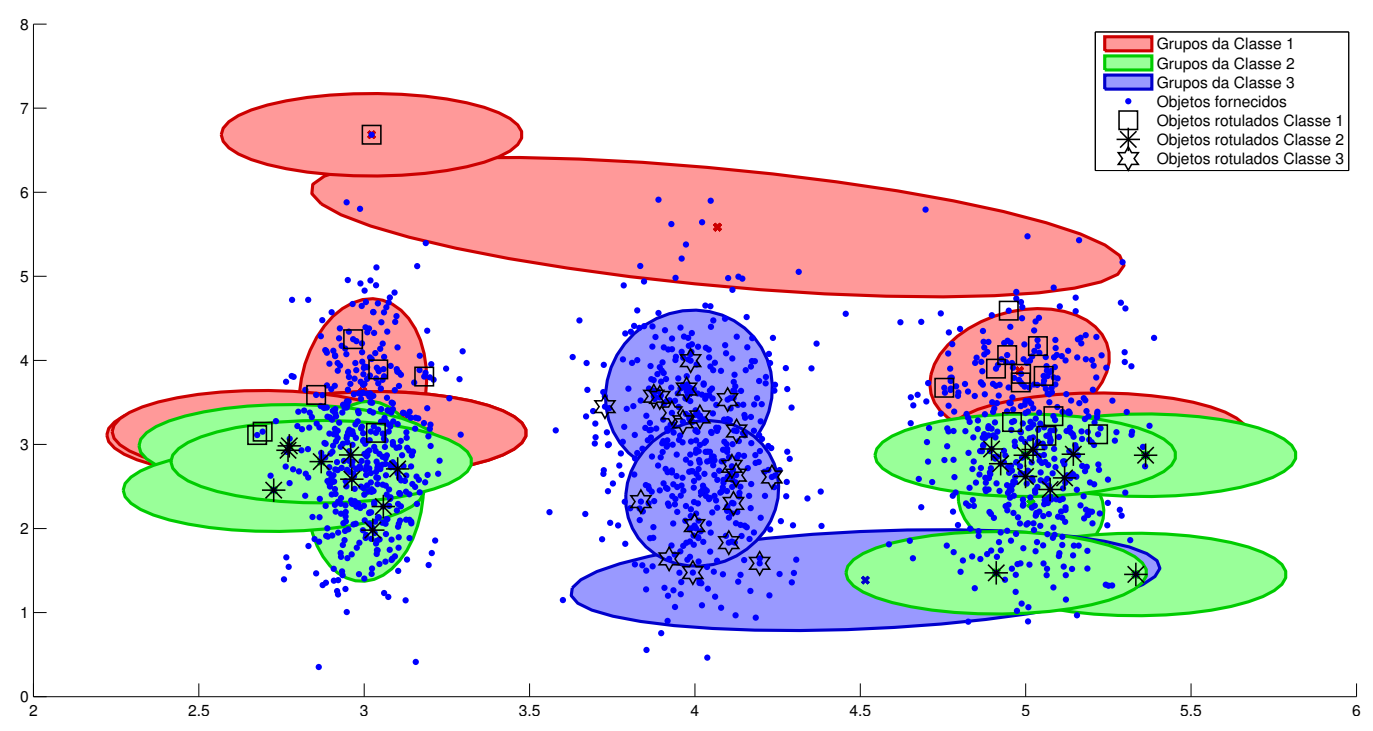

Figura 7.4: Indivíduo feasible gerado após a criação de novos grupos.

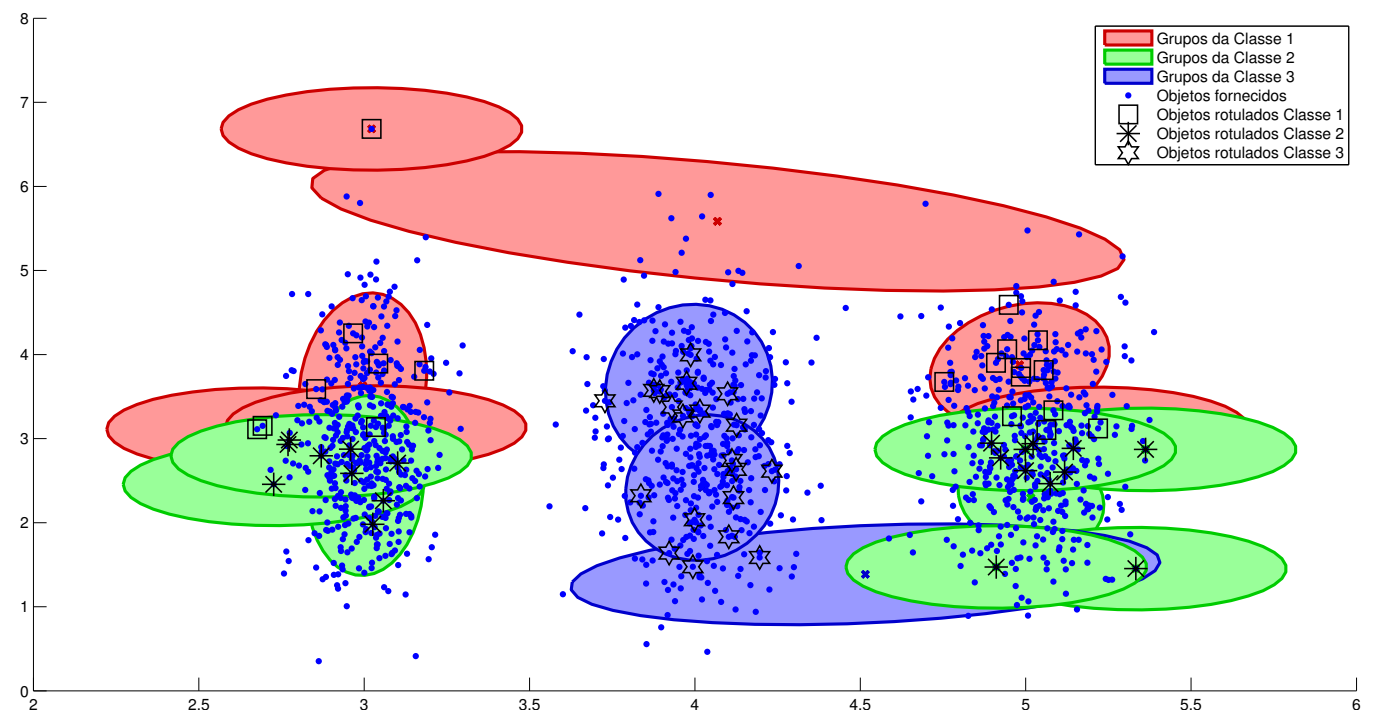

Figura 7.5: Indivíduo feasible após remoção de grupos vazios. 


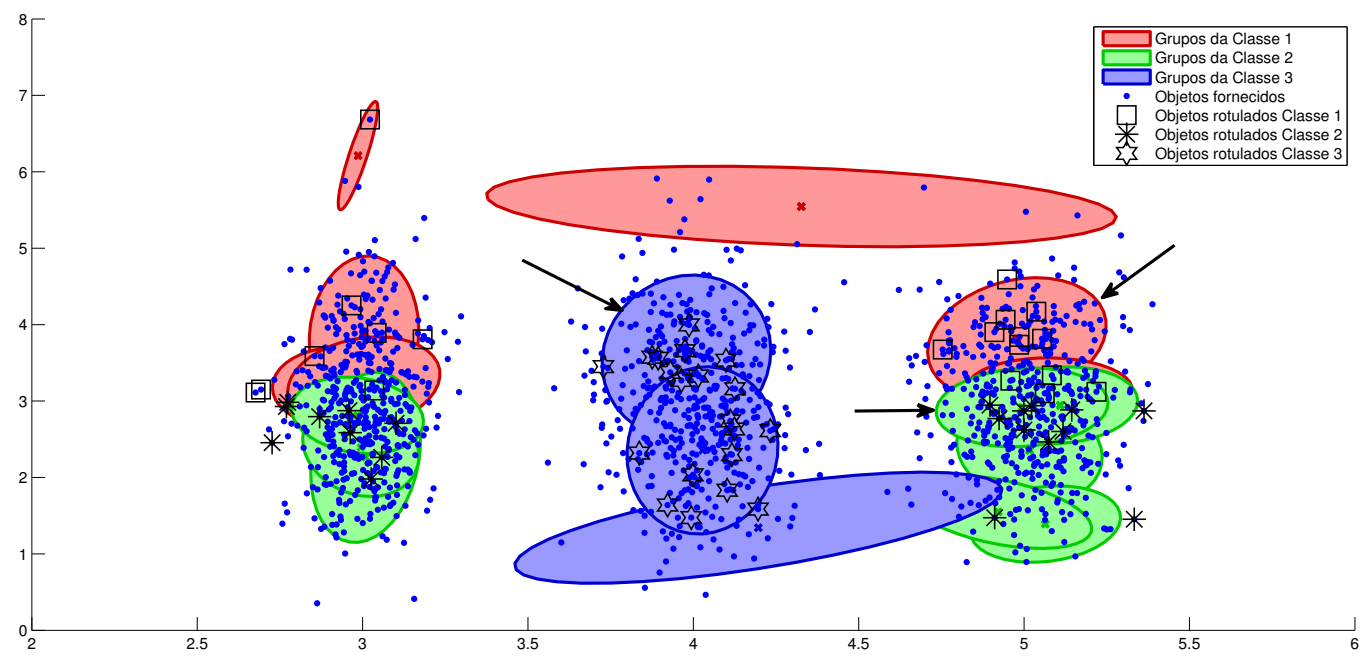

Figura 7.6: Indivíduo feasible com grupos a serem removidos indicados.

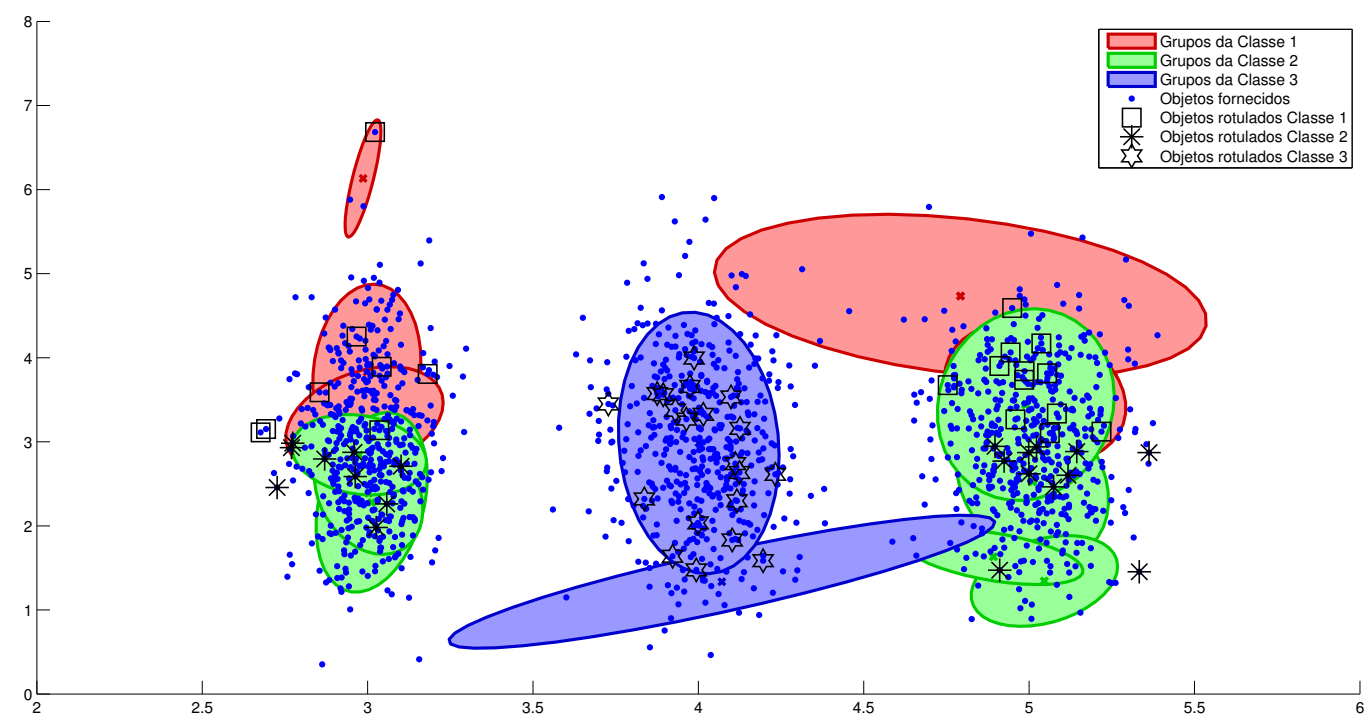

Figura 7.7: Indivíduo infeasible gerado a partir de indivíduo feasible.

soluções feasible é executado. É determinado, baseado no número de grupos do GMM, que o operador de eliminação será aplicado. Utilizando o critério PLL, são selecionados três grupos para remoção indicados por setas na Figura 7.6. Nesta figura é possível observar que o GMM já representa uma versão sensivelmente melhor em relação à sua versão anterior (Figura 7.3).

Após a remoção dos grupos uma nova etapa de refinamento é realizada, e o GMM resultante acaba por violar restrições, o que pode ser facilmente identificado via inspeção visual na região próximo ao ponto $x=5$ e $y=4$ na Figura 7.7. É importante ressaltar que embora um indivíduo infeasible tenha sido gerado, o AE está progredindo em relação à modelagem dos dados. Esta nova solução já possui menos grupos do que a solução infeasible inicial que a deu origem. Essa troca de indivíduos entre as duas populações permite ao FIECE-EM explorar melhor o espaço de busca, uma vez que frequentemente é inserido diversidade em ambas as populações, tanto feasible quanto infeasible. 


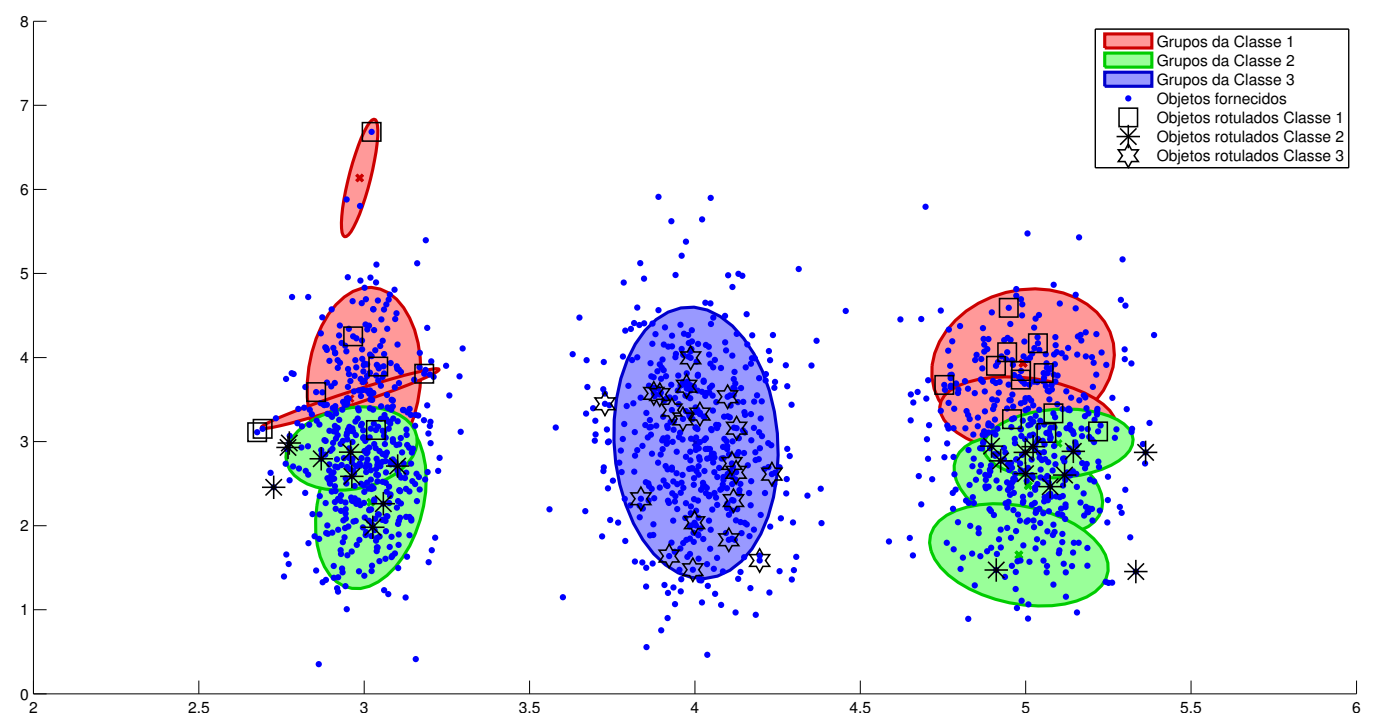

Figura 7.8: Modelo final obtido pelo FIECE-EM após convergência.

Após a convergência do algoritmo é retornado um GMM capaz de atender todas as restrições e que, idealmente, tenha bom ajuste aos dados. O GMM obtido é apresentado na Figura 7.8. Pode-se observar que na região de fronteira de decisão entre as Classes 1 e 2, o algoritmo obteve componentes de classes distintas com alguma sobreposição, conforme esperado. Com um número maior de objetos rotulados estas fronteiras poderiam ser melhor modeladas.

\subsection{Análise Empírica}

O algoritmo FIECE-EM foi comparado com o algoritmo proposto em Raghuram et al. (2014), denominado Multiple Components Parameterized (MCP $)^{3}$ — descrito na Seção 4.3.2.1. Esse algoritmo foi escolhido por ser o algoritmo da literatura mais semelhante ao FIECE-EM e ter apresentado bons resultados quando comparados com outros algoritmos da literatura (Zhao e Miller, 2005; Raghuram et al., 2014). Como baseline de comparação foi utilizado o classificador Naïve Bayes (NB) (Bishop, 2006) recebendo como conjunto de treinamento apenas os objetos envolvidos em restrições. Essa comparação permite avaliar o ganho no uso de algoritmos capazes de utilizar objetos não rotulados no aprendizado do modelo.

Para avaliar o algoritmo FIECE-EM foram consideradas 8 bases de dados do repositório UCI (Asuncion e Newman, 2007). Um sumário destas bases de dados é apresentado na Tabela 7.1. Para as bases de dados Letters e Pendigits o conjunto de atributos foi reduzido utilizando Principal Component Analysis (PCA) (Bishop, 2006). Foram selecionados os componentes principais responsáveis por $90 \%$ de variância. Esta redução no número de atributos evita problemas de condicionamento da matriz de covariância devidos a correlação entre os atributos. Assim como realizado no Capítulo 6, apenas os objetos das classes 3, 8 e 9 (I, J e L) foram considerados para a base Pendigits (Letters). O número de atributos resultantes é denotado por

\footnotetext{
${ }^{3} \mathrm{~A}$ implementação do algoritmo foi gentilmente cedida pelo autor.
} 
$M_{P C A}$ na Tabela 7.1.

Em ambos os algoritmos, o número máximo de grupos $\left(K_{\max }\right)$ foi definido como $\sqrt{N}$. No algoritmo MCP o valor de $\beta$ foi selecionado usando a estratégia descrita em Raghuram et al. (2014). Especificamente, são gerados modelos para diferentes valores de $\beta$, considerando apenas os que possuem menores valores de penalização, é selecionado o modelo com melhor ajuste aos dados. Entre os valores possíveis de $\beta$ foram considerados $\{10,50,100,500,1000,5000\}$. No algoritmo FIECE-EM foram utilizados 20 indivíduos na população feasible e 20 na população infeasible. O algoritmo foi executado por até 100 gerações, sendo interrompido caso não houvesse melhoras no melhor indivíduo da população feasible em 20 gerações. Para a inicialização dos GMMs foram executadas 2 iterações do algoritmo $K$-means. Na etapa de refinamento 5 iterações do EM foram aplicadas a cada indivíduo.

Tabela 7.1: Bases de dados usadas nos experimentos.

\begin{tabular}{ccccc}
\hline Base de dados & $\mathbf{N}$ & $\mathbf{M}$ & $\mathbf{M}_{P C A}$ & \# Classes \\
\hline Banknote & 1.372 & 4 & - & 2 \\
Breast Cancer & 683 & 9 & - & 2 \\
Ionosphere & 351 & 34 & - & 2 \\
Iris & 150 & 4 & - & 3 \\
Letters & 2,263 & 16 & 7 & 3 \\
Pendigits & 3,165 & 16 & 7 & 3 \\
Wholesale & 440 & 6 & - & 2 \\
Wine & 178 & 13 & - & 3 \\
\hline
\end{tabular}

Para a geração de restrições, foram considerados casos em que o número de objetos rotulados por classe pertence ao conjunto $\{3,4,5,6,7,10,15,20,25,30\}$. De cada conjunto de objetos rotulados, todas as restrições $\overline{M L}$ e $\overline{C L}$ são deduzidas. Os objetos usados para derivar as restrições foram selecionados aleatoriamente. Todo o processo foi repetido 10 vezes a fim de se obter melhores estimativas das estatísticas de interesse.

As análises foram divididas em duas etapas. Na primeira etapa, as partições obtidas por cada algoritmo foram comparadas, enquanto que na segunda etapa, o impacto da quantidade de supervisão fornecida no erro de classificação é analisado.

\subsubsection{Comparando as Partições dos Dados}

A qualidade das partições obtidas foi avaliada utilizando o Adjusted Rand Index (ARI) (Hubert e Arabie, 1985; Vinh et al., 2010), comparando as partições de referência fornecidas pelas classes com as obtidas por cada algoritmo. As médias de ARI obtidas por cada algoritmo em cada base são apresentadas nas Figuras 7.9(a)-7.9(h). Em linhas gerais, os algoritmos obtiveram resultados semelhantes nas diferentes bases de dados (com exceção das bases de dados Breast Cancer e Ionosphere), sendo uma característica recorrente o MCP obter melhores resultados quando o número de objetos rotulados por classe é pequeno $(<7)$. Estes resultados são animadores, em especial, pelo fato do FIECE-EM não possuir um parâmetro de compromisso entre 
Tabela 7.2: Número médio de grupos por classe estimados pelo FIECE-EM e MCP (considerando todas as quantidades de objetos rotulados).

\begin{tabular}{|c|c|c|c|c|c|c|c|c|c|c|}
\hline \multirow{2}{*}{$\begin{array}{c}\text { Base de dados } \\
\text { Classe }\end{array}$} & \multicolumn{3}{|c|}{ Letters } & \multicolumn{3}{|c|}{ Wine } & \multicolumn{2}{|c|}{ Banknote } & \multicolumn{2}{|c|}{ Ionosphere } \\
\hline & I & $\mathbf{J}$ & $\mathbf{L}$ & C1 & $\mathrm{C2}$ & C3 & $\mathbf{0}$ & 1 & Good & Bad \\
\hline \# Grupos FIECE-EM & 11 & 10 & 10 & 2 & 3 & 2 & 17 & 15 & 3 & 3 \\
\hline \# Grupos MCP & 6 & 5 & 5 & 2 & 2 & 2 & 6 & 6 & 2 & 2 \\
\hline Base de dados & \multicolumn{3}{|c|}{ Iris } & \multicolumn{3}{|c|}{ Pendigits } & \multicolumn{2}{|c|}{ Breast Cancer } & \multicolumn{2}{|c|}{ Wholesale } \\
\hline Classe & Setosa & Versicolor & Virginica & \#3 & \#8 & \#9 & Benign & Malign & Horeca & Retail \\
\hline \# Grupos FIECE-EM & 2 & 2 & 2 & 7 & 8 & 8 & 6 & 4 & 10 & 10 \\
\hline \# Grupos MCP & 2 & 2 & 2 & 4 & 6 & 6 & 2 & 2 & 3 & 3 \\
\hline
\end{tabular}

qualidade do modelo e violação dos restrições. É importante ressaltar que, os resultados obtidos pelo MCP são decorrentes da otimização do parâmetros $\beta$, e que tal tarefa é recorrente em qualquer aplicação do mesmo. Ao se considerar casos específicos, os resultados nas bases de dados Breast Cancer, Ionosphere e Letters são exemplos de problemas em que forçar a atender às restrições foi benéfico ao FIECE-EM. O algoritmo MCP obteve resultados piores em quase todos os cenários, mesmo com o aumento do número de objetos rotulados por classe. Das oito bases de dados analisadas, apenas em uma (Wine) o MCP obteve resultados consistentemente melhores que o FIECE-EM.

A Tabela 7.2 mostra os números de grupos médios por classe que cada algoritmo encontrou. $\mathrm{O}$ algoritmo FIECE-EM consistentemente obteve um número maior de grupos por classe em relação ao MCP. Isto é uma consequência esperada da filosofia do FIECE-EM de criar grupos quando não for possível atender determinadas restrições. Uma abordagem para tratar esta característica é fazer uso de uma análise posterior de um especialista de domínio sobre os grupos encontrados, a qual pode permitir encontrar restrições ruidosas, resultando em um modelo de aprendizado ativo.

\subsubsection{Avaliando o Erro de Classificação}

Nesta seção, foi avaliado o erro de classificação obtido considerando as partições encontradas pelos algoritmos como se estas fossem resultados obtidos por classificadores. O erro de classificação do NB foi utilizado como baseline de comparação. Conforme mencionado na Seção 6.3.2, apesar de classificação semissupervisionada não ser o foco principal deste trabalho, esta medida é útil por permitir mensurar o impacto da supervisão no processo de agrupamento.

A Tabela 7.3 apresenta os erros médios obtidos usando 3, 7 e 30 objetos rotulados por classe para gerar as restrições. Ao se comparar a redução de erro obtida pelo FIECE-EM com a obtida pelo MCP quando 7 objetos rotulados por classe são fornecidos ao invés de 3, observase que o FIECE-EM obteve uma redução maior na média (6\% contra 5\% do MCP), embora não seja uma diferença significativa. No entanto, ao se fazer a mesma análise considerando o uso de 30 objetos rotulados por classe e 3 objetos rotulados, a diferença entre os algoritmos é evidenciada. Especificamente, a redução de erro média obtida pelo FIECE-EM é de $11 \%$ contra $6 \%$ do MCP. É importante ressaltar que estas estatísticas são obtidas considerando-se apenas 


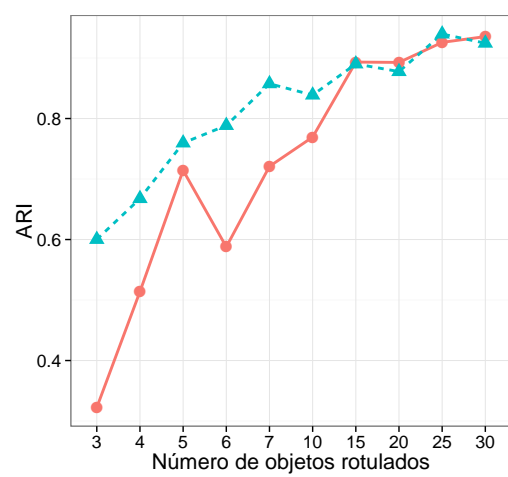

(a) Banknote

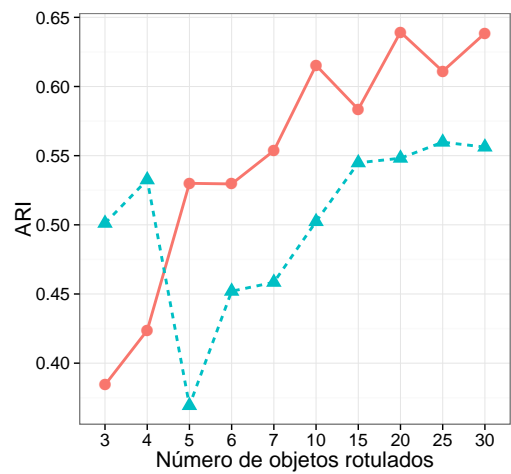

(c) Ionosphere

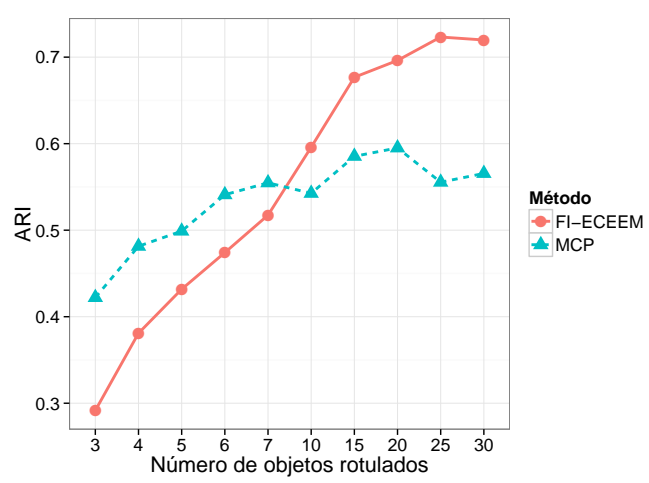

(e) Letters

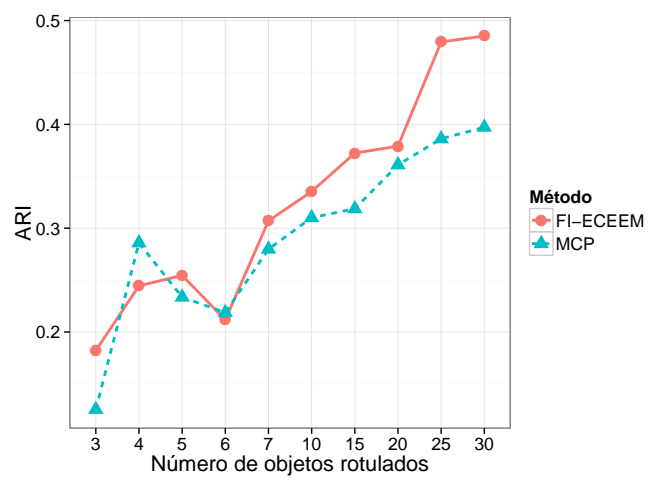

(g) Wholesale

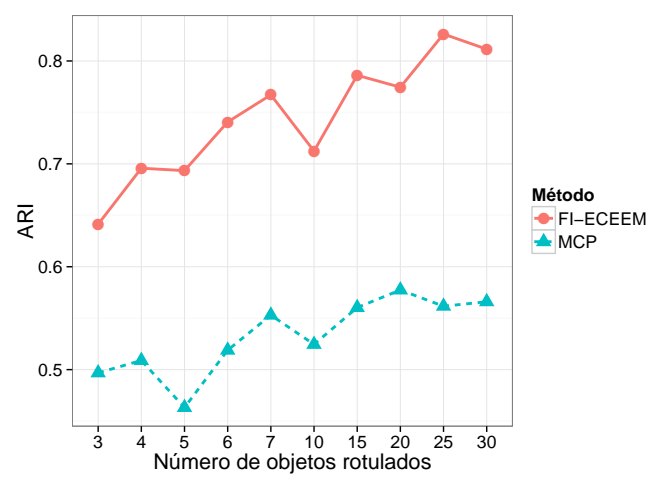

(b) Breast Cancer

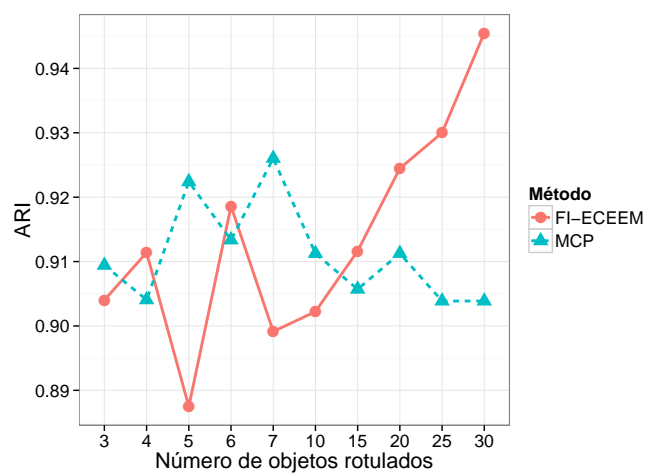

(d) Iris

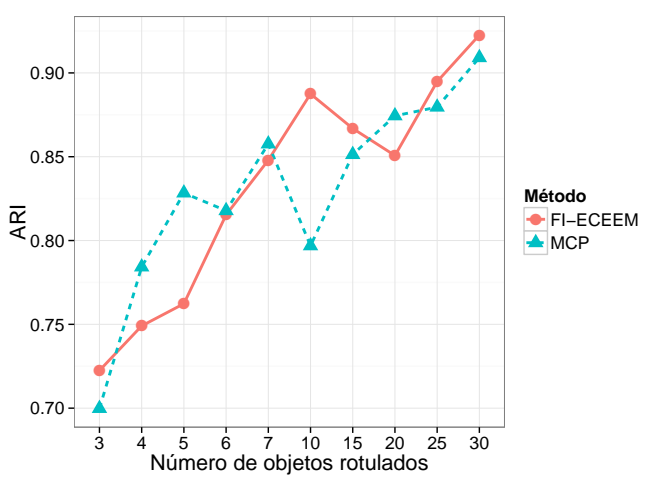

(f) Pendigits

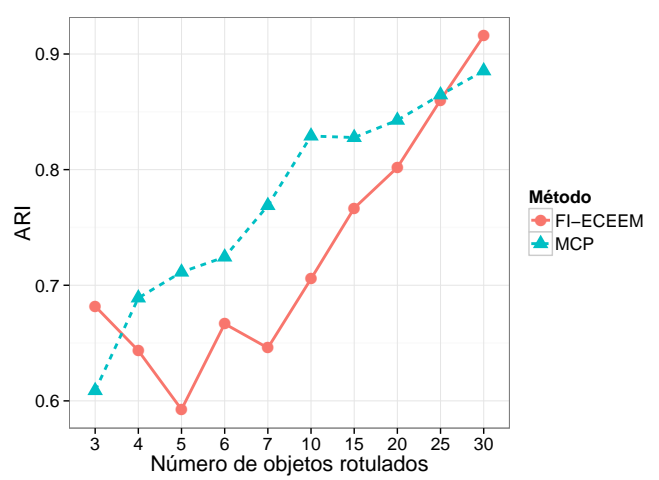

(h) Wine

Figura 7.9: Valores médios de ARI obtidos para cada quantidade de objetos rotulados por classe. 
Tabela 7.3: Taxas de erro médias (\%) para diferentes quantidades de objetos rotulados por classe (\# OR).

\begin{tabular}{|c|c|c|c|c|c|c|c|c|c|}
\hline \multirow{2}{*}{$\begin{array}{l}\text { Algoritmo } \\
\text { \#OR }\end{array}$} & \multicolumn{3}{|c|}{ FIECE-EM } & \multicolumn{3}{|c|}{ MCP } & \multicolumn{3}{|c|}{ NB } \\
\hline & 3 & 7 & 30 & 3 & 7 & 30 & 3 & 7 & 30 \\
\hline Banknote & 23.52 & 8.35 & 1.74 & 13.98 & 3.81 & 2.04 & 37.18 & 20.50 & 16.42 \\
\hline Breast Cancer & 10.15 & 6.22 & 5.01 & 14.76 & 13.18 & 13.07 & 15.73 & 14.36 & 6.58 \\
\hline Ionosphere & 21.59 & 13.56 & 12.03 & 15.10 & 18.01 & 13.30 & 40.75 & 24.24 & 13.54 \\
\hline Iris & 3.55 & 4.19 & 4.67 & 3.33 & 2.87 & 4.00 & 22.34 & 6.20 & 5.67 \\
\hline Letters & 34.17 & 19.98 & 10.78 & 25.75 & 18.96 & 20.37 & 46.02 & 30.79 & 21.44 \\
\hline Pendigits & 10.94 & 5.41 & 2.73 & 12.71 & 5.08 & 3.20 & 31.65 & 8.06 & 4.55 \\
\hline Wholesale & 30.37 & 23.87 & 17.61 & 32.14 & 24.30 & 21.39 & 34.70 & 13.83 & 11.82 \\
\hline Wine & 12.54 & 16.31 & 3.86 & 18.88 & 9.55 & 5.23 & 26.21 & 8.92 & 3.52 \\
\hline
\end{tabular}

Tabela 7.4: Tabela de vitórias/empates/derrotas entre os algoritmos para diferentes quantidades de objetos rotulados por classe (\# OR) considerando as taxas de erro médias.

\begin{tabular}{|c|c|c|c|c|c|c|c|c|c|}
\hline \multirow{2}{*}{$\begin{array}{c}\text { \#OR } \\
\text { Algoritmo }\end{array}$} & \multicolumn{3}{|c|}{3} & \multicolumn{3}{|c|}{7} & \multicolumn{3}{|c|}{30} \\
\hline & FIECE-EM & МСР & NB & FIECE-EM & МСР & NB & FIECE-EM & МCP & NB \\
\hline FIECE-EM & - & $4 / 0 / 4$ & $8 / 0 / 0$ & - & $3 / 0 / 5$ & $6 / 0 / 2$ & - & $7 / 0 / 1$ & $6 / 0 / 2$ \\
\hline МCP & $4 / 0 / 4$ & - & $8 / 0 / 0$ & $5 / 0 / 3$ & - & $6 / 0 / 2$ & $1 / 0 / 7$ & - & $5 / 0 / 3$ \\
\hline NB & $0 / 0 / 8$ & $0 / 0 / 8$ & - & $2 / 0 / 6$ & $2 / 0 / 6$ & - & $2 / 0 / 6$ & $3 / 0 / 5$ & - \\
\hline
\end{tabular}

os objetos não utilizados nas restrições, o que sugere que o FIECE-EM ao ser rígido em relação à atender as restrições foi capaz de obter uma melhor propagação do efeitos das restrições do que o MCP. Os resultados na base de dados Letters reforça essa observação. Inicialmente, com 3 objetos rotulados por classe, o FIECE-EM obteve um erro $32 \%$ maior do que o erro obtido pelo MCP. No entanto, quando 30 objetos rotulados por classe foram utilizados para extrair as restrições, o erro médio obtido pelo FIECE-EM foi aproximadamente $48 \%$ menor do que o erro obtido pelo MCP. Ao se comparar com os resultados obtidos pelo NB, pode-se observar que com exceção das bases de dados Wholesale e Wine, o algoritmo FIECE-EM obteve resultados melhores para todos os cenários de número de objetos rotulados. Tal fato evidencia o benefício de se obter modelos preditivos fazendo uso de objetos não rotulados, mesmo quando apenas uma pequena porção destes está disponível. Os números de vitórias/empates/derrotas obtidos ao se comparar os resultados de cada algoritmo são sumarizados na Tabela 7.4. Por exemplo, a $4^{\mathrm{a}}$ linha e $9^{\mathrm{a}}$ coluna da tabela indica que, considerando o cenário em que 30 objetos rotulados por classe foram utilizados, o algoritmo FIECE-EM obteve resultados melhores que o MCP em 7 bases de dados, não houve resultados iguais e o algoritmo MCP obteve resultados melhores que o FIECE-EM em apenas 1 base de dados. Nesta tabela pode-se observar que o algoritmo FIECE-EM é competitivo com o algoritmo MCP, conseguindo superá-lo quando o número de restrições é alto na maioria dos casos.

\subsection{Considerações Finais}

Neste capítulo, foi apresentado o algoritmo Feasible-Infeasible Evolutionary Create \& Eliminate Algorithm for Expectation Maximizations (FIECE-EMs) para ADR. Este AE é resultado 
de uma combinação do algoritmo Feasible-Infeasible Two-Population (FI2POP), que apresenta bons resultados na literatura de AEs para problemas com restrições, e da filosofia do algoritmo Multiple Clusters per Class K-means (MCCK) - apresentado no Capítulo 6. O FIECE-EM tem como vantagem, em relação aos algoritmos similares da literatura, o fato de não possuir um parâmetro de compromisso entre qualidade do modelo e desejo de atender restrições. Embora possua parâmetros comuns de AEs, e.g., tamanho da população e número de gerações, estes podem ser definidos de acordo com os recursos computacionais disponíveis.

Experimentos realizados em 8 bases de dados evidenciam que o algoritmo FIECE-EM é capaz de obter resultados pelo menos competitivos com o algoritmo Multiple Components Parameterized (MCP) (Raghuram et al., 2014), que busca incorporar as restrições no modelo gerador da base de dados. As análises descritas estão sendo estendidas para uma posterior publicação em periódico internacional. 


\section{CAPÍTULO}

\section{Conclusões e Trabalhos futuros}

Diferentes áreas de pesquisa foram envolvidas nesta tese. A Figura 8.1 sumariza tais áreas indicando o fluxo do desenvolvimento desta tese. As áreas específicas em que as contribuições desta tese estão inclusas encontram-se com fundo realçado. Em linhas específicas, buscou-se confirmar as hipóteses levantadas no Capítulo 1. Conforme abordado na Seção 1.4, a Hipótese 1 levantada nessa tese é:

Hipótese 1: Um Algoritmo Evolutivo (AE), desenvolvido considerando estratégias bem conhecidas da literatura de algoritmos de busca local, é capaz de estimar os parâmetros de um Modelo de Mistura de Gaussianas (GMM), tendo como conhecimento a priori apenas os dados e sem nenhuma imposição estrutural sob o modelo, de forma eficiente e competitiva com abordagens já existentes para a estimação de parâmetros.

Com o intuito de confirmar esta hipótese, foi proposto o algoritmo Evolutionary Split \& Merge Algorithm for Expectation Maximization (ESM-EM). Este algoritmo combina propostas existentes na literatura para dividir e unir componentes de modelos de mistura - Split and

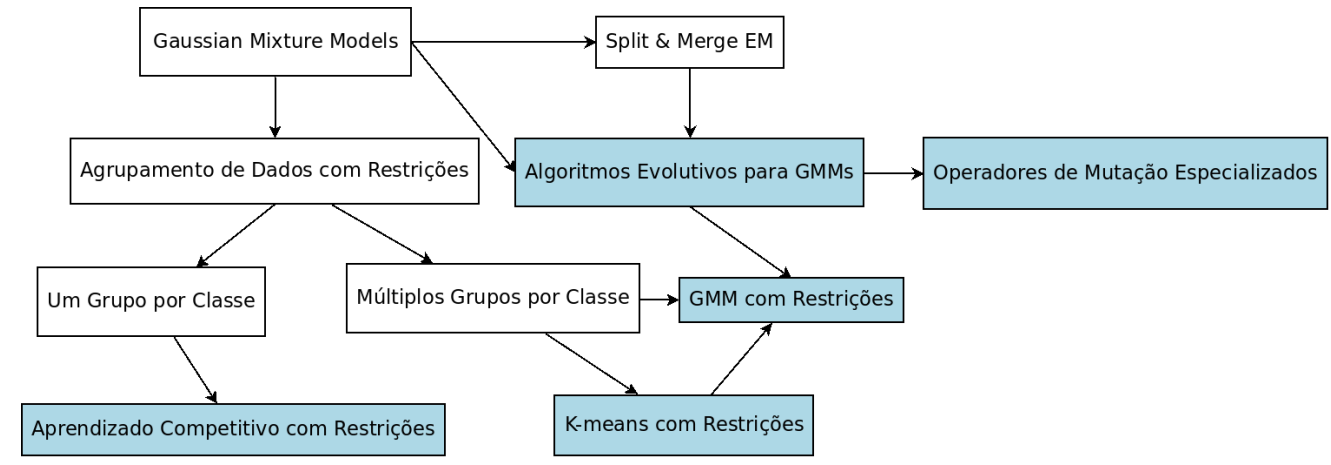

Figura 8.1: Fluxo do desenvolvimento desta tese. 
Merge (SM) (Zhang et al., 2003) e AEs. Diversas variantes do ESM-EM foram propostas e analisadas em um conjunto de 35 bases de dados. A melhor variante foi então selecionada e comparada com o algoritmo Genetic-based Expectation Maximization (GA-EM) (Pernkopf e Bouchaffra, 2005), que é considerado um algoritmo estado-da-arte em AE para GMM. Por meio de uma análise extensa de experimentos, mostrou-se que o ESM-EM é capaz de obter resultados competitivos, em termos de acurácia e eficiência, com o GA-EM, mesmo quando os parâmetros do GA-EM são (previamente) otimizados. Considerando todos os resultados obtidos, apresentados no Capítulo 3, a hipótese 1 desta tese foi confirmada. Resultados preliminares destas análises foram divulgados em Covões e Hruschka (2011). A análise completa dos resultados obtidos foi documentada em um artigo científico que atualmente está em revisão em um periódico internacional.

Para completar a Hipótese 1, foi formulada a segunda hipótese dessa tese:

Hipótese 2: Um algoritmo de ADR que processa os dados de forma online é capaz de obter acurácia comparável com algoritmos da literatura de ADR que processam os dados em lotes (batches).

Para validar essa segunda hipótese, foram propostos dois algoritmos (Online Linear Constrained Vector Quantization Error (O-LCVQE) e Constrained Rival Penalized Competitive Learning (C-RPCL)). Esses algoritmos foram então avaliados via experimentos realizados com 8 bases de dados comumente utilizadas para avaliar algoritmos de agrupamento não supervisionado e semissupervisionado. Além destas bases de dados, foi considerada também a base de dados do KDDCUP'99 de Detecção de Intrusão em redes que costuma ser utilizada para avaliar algoritmos de agrupamento de streams, cenário no qual, usualmente, os dados são processados apenas uma vez. Estes experimentos demonstraram que o algoritmo C-RPCL não apenas apresenta melhores resultados que o O-LCVQE como também fornece partições melhores que o algoritmo de processamento em lote LCVQE. Desta forma, os resultados obtidos oferecem boas evidências de que a hipótese 2 pode ser confirmada. Os algoritmos propostos e as análises descritas no Capítulo 5 foram publicados em Covões et al. (2013a).

Finalmente, baseando-se nos desenvolvimentos proporcionados pelas hipóteses 1 e 2, foi investigada a terceira hipótese dessa tese:

Hipótese 3; É possível desenvolver algoritmos para ADR com as seguintes propriedades: (i) capacidade de lidar com situações de mais de um grupo por classe, mesmo que recebendo como supervisão restrições entre pares de objetos; (ii) ausência de parâmetros críticos de compromisso entre qualidade de soluções e rigidez de restrições; (iii) desobrigação de que o usuário defina o número de grupos por classe a priori; (iv) eficiência computacional.

Para validar essa terceira hipótese, dois algoritmos foram propostos: Multiple Clusters per Class K-means (MCCK) e Feasible-Infeasible Evolutionary Create \& Eliminate Algorithm for 
Expectation Maximization (FIECE-EM) (Capítulos 6 e 7, respectivamente). O primeiro algoritmo baseia-se no $K$-means e, portanto, considera que os componentes do GMM possuem matrizes de covariância cujos elementos são iguais e proporcionais à matriz identidade. Já o algoritmo FIECE-EM não possui premissas em relação às matrizes de covariâncias, i.e., elas são irrestritas. Foi demonstrado, via experimentos empíricos, que ambos os algoritmos são capazes de obter resultados iguais ou melhores do que algoritmos bem conhecidos da literatura (Basu et al., 2002; Raghuram et al., 2014). Além disso, as análises realizadas comprovam que a premissa de um grupo por classe não é válida em diversos casos, mesmo em bases de benchmark da literatura. Por tal razão, algoritmos com essa capacidade de estimar o número de grupos por classe são relevantes para diversas aplicações de Mineração de Dados. Finalmente, tais algoritmos não possuem parâmetros de compromisso entre qualidade e rigidez de restrições. Devido ao uso do algoritmo $K$-means, que é sabidamente escalável, o MCCK pode ser considerado um algoritmo eficiente - mesmo que com o tratamento de grupos redundantes seja adicionado um custo computacional de $O\left(K^{2}\right)$, sendo $K$ o número de grupos. No caso do FIECE-EM, o custo de evoluir duas populações que tratam o GMM com matrizes de covariância irrestritas não é desprezível. No entanto, devido ao processamento dos indivíduos ser independente do restante da população, é possível implementá-lo de forma paralela e distribuída - e.g., usando MapReduce (Dean e Ghemawat, 2004, 2008) — sem mudanças significativas.

Conforme se pode constatar, as hipóteses levantadas na tese foram apropriadamente validadas, originando contribuições relevantes para a área de Mineração de Dados. Além das contribuições diretamente vinculadas às hipóteses levantadas na tese, os desenvolvimentos realizados suscitaram contribuições adicionais indiretamente relacionadas aos objetivos da tese: um AE baseado na eliminação e criação de componentes de GMMs, publicado em Covões e Hruschka (2013); um estudo comparativo de algoritmos de ADR, publicado em Covões et al. (2013c); a combinação do algoritmo MCCK com máquinas de vetores de suporte transdutivas, publicado em Covões et al. (2013b).

\subsection{Trabalhos Futuros}

Como trabalhos futuros promissores, destacam-se: (i) implementações paralelas e distribuídas dos AEs propostos; (ii) extensão das análises empíricas para bases de dados de aplicações reais; (iii) análise de sensibilidade dos parâmetros dos algoritmos de aprendizado competitivo. Na sequência, cada uma destas frentes de pesquisa são discutidas.

\subsubsection{Implementações Paralelas e Distribuídas}

Os algoritmos ESM-EM e FIECE-EM (Capítulos 3 e 7) modificam cada indivíduo isoladamente dos demais. Esta propriedade permite que tais algoritmos sejam implementados de forma paralela e distribuída e sem a necessidade de modificações significativas. Por exemplo, 
considerando o paradigma MapReduce (Dean e Ghemawat, 2004, 2008), seria possível realizar a distribuição das operações de refinamento e mutação entre os nós de um cluster de computadores na fase map, enquanto que a fase reduce realizaria a seleção dos indivíduos.

Um estudo sobre o desempenho de tais algoritmos, neste paradigma, bem como uma análise sobre qual tamanho a base de dados deve ter para que a melhora na eficiência computacional seja significativa, pode permitir aumentar a utilidade prática desses algoritmos, em particular, porque há uma certa crença de que AEs serem frequentemente preteridos devido ao custo computacional.

\subsubsection{Extensão de Análises Empíricas}

GMMs são utilizados em diversas aplicações como reconhecimento de dígitos manuscritos (Chen et al., 2011), segmentação de imagens (Zhang et al., 2003) e análise de imagens de ressonância magnética do cérebro (Tohka et al., 2007). A avaliação da acurácia dos algoritmos propostos nestes cenários específicos é uma frente de pesquisa promissora. Devido ao amplo interesse em tais aplicações, existem bases de dados de benchmark reconhecidas na literatura para cada uma delas. Especificamente, a base de dados MNIST ${ }^{1}$ (LeCun et al., 1998) de reconhecimento de dígitos, a coleção BSD500² (Arbelaez et al., 2011) para segmentação de imagens e os repositórios BrainWeb ${ }^{3}$ (Cocosco et al., 1997) e OpenfMRI $I^{4}$ para análise estrutural e funcional de imagens de ressonância magnética são amplamente usadas. Tais bases de dados são ótimos pontos de partida para a avaliação de algoritmos nestas áreas.

Tanto no cenário não supervisionado quanto semissupervisionado, os algoritmos propostos nesta tese podem ser úteis nas aplicações mencionadas. A aplicação deles como modelos independentes ou como combinações com outros algoritmos é de interesse. Além disso, é promissor avaliar a combinação dos algoritmos aqui propostos com aprendizado ativo em aplicações específicas. Este tipo de análise é de grande valia em aplicações práticas devido à minimização do esforço de um especialista de domínio.

\subsubsection{Melhorias nos Algoritmos e Análises de Aprendizado Competitivo}

Nas análises realizadas no Capítulo 5, foram utilizados para os parâmetros de taxa de aprendizado $(\alpha)$ e taxa de esquecimento $(\beta)$ valores previamente estabelecidos em pesquisas bemconhecidas da literatura. No entanto, é interessante avaliar a sensibilidade dos algoritmos em relação a estes parâmetros. Além disso, o estudo de heurísticas que possam funcionar em casos frequentes é interessante. Outra frente de pesquisa promissora é a adaptação dos algoritmos O-LCVQE e C-RPCL para o contexto de múltiplos grupos por classe. No entanto, para tal serão necessárias alterações significativas nos algoritmos. Por fim, um estudo mais profundo do uso

\footnotetext{
${ }^{1}$ http://yann.lecun.com/exdb/mnist/

${ }^{2} \mathrm{http} / / / \mathrm{www} . e e c s . b e r k e l e y . e d u /$ Research/Projects/CS/vision/grouping/resources.html

${ }^{3}$ http://brainweb.bic.mni.mcgill.ca/brainweb/

${ }^{4} \mathrm{https}: / /$ openfmri.org/
} 
de tais algoritmos em bases de dados reais de larga escala, em especial em relação ao número de objetos, é de interesse. 


\section{Referências Bibliográficas}

Abou-Nasr, M., Lessmann, S., Stahlbock, R., e Weiss, G. (2014). Real World Data Mining Applications. Annals of Information Systems. Springer International Publishing. (Citado na página 3.)

Acharya, A., Hruschka, E. R., Ghosh, J., e Acharyya, S. (2014). An optimization framework for combining ensembles of classifiers and clusterers with applications to nontransductive semisupervised learning and transfer learning. ACM Transactions on Knowledge Discovery from Data, 9(1):1:1-1:35. (Citado na página 6.)

Aggarwal, C. e Reddy, C. (2013). Data Clustering: Algorithms and Applications. Chapman $\&$ Hall/CRC Data Mining and Knowledge Discovery Series. Taylor \& Francis. (Citado nas páginas 4 e 5.)

Aggarwal, C. C. (2014). Data Classification: Algorithms and Applications. Chapman \& Hall/CRC. (Citado na página 3.)

Aggarwal, C. C., Han, J., Wang, J., e Yu, P. S. (2003). A framework for clustering evolving data streams. Em $V L D B$, páginas 81-92. (Citado na página 86.)

Aha, D. W., Kibler, D. F., e Albert, M. K. (1991). Instance-based learning algorithms. Machine Learning, 6:37-66. (Citado nas páginas 3 e 90.)

Ahalt, S. C., Krishnamurthy, A. K., Chen, P., e Melton, D. E. (1990). Competitive learning algorithms for vector quantization. Neural Networks, 3(3):277 - 290. (Citado na página 78.)

Aloise, D., Deshpande, A., Hansen, P., e Popat, P. (2009). NP-hardness of euclidean sum-ofsquares clustering. Machine Learning, 75:245-248. (Citado na página 5.)

Alves, V., Campello, R., e Hruschka, E. (2006). Towards a fast evolutionary algorithm for clustering. Em IEEE Congress on Evolutionary Computation (CEC'06), páginas 1776-1783. IEEE Press. (Citado nas páginas 22, 24 e 25.) 
Anderson, D. T., Bezdek, J. C., Popescu, M., e Keller, J. M. (2010). Comparing fuzzy, probabilistic, and possibilistic partitions. IEEE Transactions on Fuzzy Systems, 18:906-918. (Citado nas páginas 3 e 4.)

Anderson, T. W. e Bahadur, R. R. (1962). Classification into two Multivariate Normal Distributions with Different Covariance Matrices. The Annals of Mathematical Statistic, 33(2):420431. (Citado na página 16.)

Arbelaez, P., Maire, M., Fowlkes, C., e Malik, J. (2011). Contour detection and hierarchical image segmentation. IEEE Trans. Pattern Anal. Mach. Intell., 33(5):898-916. (Citado na página 118.)

Ares, M. E., Parapar, J., e Barreiro, Á. (2009). Avoiding bias in text clustering using constrained k-means and may-not-links. Em International Conference on Theory of Information Retrieval: Advances in Information Retrieval Theory (ICTIR'09), páginas 322-329, Berlin, Heidelberg. Springer-Verlag. (Citado nas páginas 62, 63 e 65.)

Arthur, D. e Vassilvitskii, S. (2007). K-means++: The advantages of careful seeding. Em Proceedings of the Eighteenth Annual ACM-SIAM Symposium on Discrete Algorithms, SODA '07, páginas 1027-1035, Philadelphia, PA, USA. Society for Industrial and Applied Mathematics. (Citado na página 65.)

Ashlock, D. A. (2006). Evolutionary computation for modeling and optimization. Springer. (Citado na página 20.)

Asuncion, A. e Newman, D. (2007). UCI machine learning repository. http: / / www . ics . uci.edu/ mlearn/MLRepository.html. (Citado nas páginas 48, 49, 81, 92 e 108.)

Bahmani, B., Moseley, B., Vattani, A., Kumar, R., e Vassilvitskii, S. (2012). Scalable kmeans++. Proc. VLDB Endow., 5(7):622-633. (Citado na página 65.)

Banerjee, A. e Ghosh, J. (2006). Scalable clustering algorithms with balancing constraints. Data Mining and Knowledge Discovery, 13(3):265-295. (Citado na página 59.)

Bar-Hillel, A., Hertz, T., Shental, N., e Weinshall, D. (2005). Learning a mahalanobis metric from equivalence constraints. Journal of Machine Learning Research, 6:937-965. (Citado nas páginas 62 e 69.)

Bartz-Beielstein, T., Lasarczyk, C., e Preuss, M. (2005). Sequential parameter optimization. Em IEEE Congress on Evolutionary Computation, páginas 773-780. (Citado na página 54.)

Basu, S., Banerjee, A., e Mooney, R. (2002). Semi-supervised clustering by seeding. Em ICML'02. (Citado nas páginas 9, 92 e 117.) 
Basu, S., Banerjee, A., e Mooney, R. J. (2004a). Active semi-supervision for pairwise constrained clustering. Em SIAM International Conference on Data Mining (SDM'04), páginas 333-344. SIAM. (Citado na página 61.)

Basu, S., Bilenko, M., e Mooney, R. J. (2004b). A probabilistic framework for semi-supervised clustering. Em ACM SIGKDD International Conference on Knowledge Discovery and Data Mining (KDD’04), páginas 59-68, New York, NY, USA. ACM. (Citado nas páginas 61 e 62.)

Basu, S., Davidson, I., e Wagstaff, K. (2008). Constrained Clustering: Advances in Algorithms, Theory, and Applications. Chapman \& Hall/CRC. (Citado nas páginas 5, 6, 59, 60, 61 e 97.)

Bayes, T. (1763). An essay towards solving a problem in the doctrine of chances. Philosophical Transactions of the Royal Society of London, 53:370-418. (Citado na página 3.)

Benabdeslem, K. e Snoussi, J. (2009). A probabilistic approach for constrained clustering with topological map. Em International Conference on Machine Learning and Data Mining in Pattern Recognition (MLDM'09), páginas 413-426, Berlin, Heidelberg. Springer-Verlag. (Citado na página 62.)

Berry, M. (2003). Survey of Text Mining : Clustering, Classification, and Retrieval. Springer. (Citado na página 5.)

Bilenko, M., Basu, S., e Mooney, R. J. (2004). Integrating constraints and metric learning in semi-supervised clustering. Em International Conference on Machine learning (ICML'04), páginas 11-, New York, NY, USA. ACM. (Citado nas páginas 61, 62, 63, 66, 69, 70, 71, 81, 89 e 92.$)$

Bishop, C. M. (2006). Pattern Recognition and Machine Learning (Information Science and Statistics). Springer-Verlag New York, Inc., Secaucus, NJ, USA. (Citado nas páginas 3, 6, $12,13,14,15,22,40,48,63$ e 108.)

Blekas, K. e Lagaris, I. E. (2007). Split-merge incremental learning (smile) of mixture models. Em International Conference on Artificial Neural Networks (ICANN'07), páginas 291-300, Berlin, Heidelberg. Springer-Verlag. (Citado nas páginas 15, 16, 17, 18, 40 e 74.)

Bouchachia, A. e Pedrycz, W. (2006). Data clustering with partial supervision. Data Mining Knowledge Discovery, 12(1):47-78. (Citado na página 62.)

Bracewell, R. (1999). The Fourier Transform \& Its Applications. McGraw-Hill Science/Engineering/Math. (Citado na página 17.)

Bäck, T., Fogel, D. B., e Michalewicz, Z. (2000a). Evolutionary Computation 1: Basic Algorithms and Operators. Institute of Physics Publishing, Bristol. (Citado nas páginas 20, 24, 25, 38 e 102.) 
Bäck, T., Fogel, D. B., e Michalewicz, Z. (2000b). Evolutionary Computation 2: Advanced Algorithms and Operators. Institute of Physics Publishing, Bristol. (Citado na página 20.)

Cai, Z., Gong, W., Ling, C. X., e Zhang, H. (2011). A clustering-based differential evolution for global optimization. Applied Soft Computing, 11(1):1363 - 1379. (Citado na página 22.)

Campello, R. J. G. B., Hruschka, E. R., e Alves, V. S. (2009). On the efficiency of evolutionary fuzzy clustering. Journal of Heuristics, 15:43-75. (Citado nas páginas 35, 36, 47, 48, 49, 81 e 92.)

Celebi, E. (2014). Partitional Clustering Algorithms. Springer International Publishing. (Citado nas páginas 4 e 5.)

Chang, D.-X., Zhang, X.-D., e Zheng, C.-W. (2009). A genetic algorithm with gene rearrangement for k-means clustering. Pattern Recognition, 42(7):1210-1222. (Citado na página 22.)

Chapelle, O., Schölkopf, B., e Zien, A., editores (2010). Semi-Supervised Learning. The MIT Press. (Citado nas páginas 5, 6 e 87.)

Chatel, D., Tommasi, M., e Denis, P. (2014). Fast gaussian pairwise constrained spectral clustering. Em 7th European Conference on Machine Learning and Principles and Practice of Knowledge Discovery in Databases (ECML/PKDD’2014), páginas 1-16. (Citado na página 62.)

Chaudhuri, D., Chaudhuri, B. B., e Murthy, C. A. (1992). A new split-and-merge clustering technique. Pattern Recognition Letters, 13:399-409. (Citado na página 15.)

Chen, B., Zhao, S., Zhu, P., e Príncipe, J. C. (2012). Quantized kernel least mean square algorithm. IEEE Trans. Neural Netw. Learning Syst., páginas 22-32. (Citado na página 77.)

Chen, C. P. e Zhang, C.-Y. (2014). Data-intensive applications, challenges, techniques and technologies: A survey on big data. Information Sciences, 275(0):314 - 347. (Citado na página 1.)

Chen, X., Liu, X., e Jia, Y. (2011). Discriminative structure selection method of gaussian mixture models with its application to handwritten digit recognition. Neurocomputing, 74(6):954 - 961. (Citado nas páginas 12 e 118.)

Choy, C. S.-T. e Siu, W.-C. (1998). A class of competitive learning models which avoids neuron underutilization problem. IEEE Transactions on Neural Networks, 9(6):1258-1269. (Citado nas páginas 77 e 78 .) 
Cocosco, C. A., Kollokian, V., Kwan, R. K.-S., Pike, G. B., e Evans, A. C. (1997). Brainweb: Online interface to a $3 \mathrm{~d}$ mri simulated brain database. NeuroImage, 5:425. (Citado na página 118.)

Coello, C. A. C. (2002). Theoretical and numerical constraint-handling techniques used with evolutionary algorithms: a survey of the state of the art. Computer Methods in Applied Mechanics and Engineering, 191(11-12):1245 - 1287. (Citado na página 98.)

Constantinopoulos, C. e Likas, A. (2007). Unsupervised learning of gaussian mixtures based on variational component splitting. IEEE Transactions on Neural Networks, 18(3):745-755. (Citado na página 14.)

Cormen, T. H., Leiserson, C. E., Rivest, R. L., e Stein, C. (2001). Introduction to Algorithms. The MIT Press, New York. (Citado na página 60.)

Costa, I. G., Schönhuth, A., Hafemeister, C., e Schliep, A. (2009). Constrained mixture estimation for analysis and robust classification of clinical time series. Bioinformatics, 25:i6-i14. (Citado na página 62.)

Covões, T., Hruschka, E., e Ghosh, J. (2013a). Competitive learning with pairwise constraints. Neural Networks and Learning Systems, IEEE Transactions on, 24(1):164-169. (Citado nas páginas $8,9,86$ e 116.)

Covões, T. F., Barros, R. C., da Silva, T. S., Hruschka, E. R., e de Carvalho, A. C. P. L. F. (2013b). Hierarchical bottom-up safe semi-supervised support vector machines for multiclass transductive learning. Journal of Information and Data Management, 4(3):357-372. (Citado nas páginas 8, 95 e 117.)

Covões, T. F. e Hruschka, E. R. (2011). Splitting and merging gaussian mixture model components: An evolutionary approach. Machine Learning and Applications, Fourth International Conference on, 1:106-111. (Citado nas páginas 8, 33, 35, 57 e 116.)

Covões, T. F. e Hruschka, E. R. (2013). Unsupervised learning of gaussian mixture models: Evolutionary create and eliminate for expectation maximization algorithm. Em Evolutionary Computation (CEC), 2013 IEEE Congress on, páginas 3206-3213. IEEE. (Citado nas páginas 8,101 e 117.)

Covões, T. F., Hruschka, E. R., e Ghosh, J. (2013c). A study of k-means-based algorithms for constrained clustering. Intelligent Data Analysis, 17. (Citado nas páginas 8, 9, 66, 75, 78, 83 e 117.)

Dasgupta, S. (1999). Learning mixtures of Gaussians. Em 40th Annual Symposium on Foundations of Computer Science, páginas 634-644. IEEE Computer Society. (Citado nas páginas 12, 48 e 49.) 
Davidson, I. e Ravi, S. S. (2005a). Agglomerative hierarchical clustering with constraints: Theoretical and empirical results. Em Knowledge Discovery in Databases: 9th European Conference on Principles and Practice of Knowledge Discovery in Databases (PKDD'05), páginas 59-70. (Citado na página 61.)

Davidson, I. e Ravi, S. S. (2005b). Clustering with constraints: Feasibility issues and the kmeans algorithm. Em SIAM International Conference on Data Mining (SDM'05), páginas 138-149. SIAM. (Citado nas páginas 60, 62, 63, 66 e 67.)

Davidson, I. e Ravi, S. S. (2006). Identifying and generating easy sets of constraints for clustering. Em National Conference on Artificial Intelligence and the Eighteenth Innovative Applications of Artificial Intelligence Conference (AAAI 2006). AAAI Press. (Citado na página 60.)

Davidson, I. e Ravi, S. S. (2009). Using instance-level constraints in agglomerative hierarchical clustering: theoretical and empirical results. Data Mining and Knowledge Discovery, 18:257-282. (Citado na página 61.)

Davidson, I., Wagstaff, K., e Basu, S. (2006). Measuring constraint-set utility for partitional clustering algorithms. Em European Conference on Principles and Practice of Knowledge Discovery in Databases (PKDD 2006), volume 4213 of Lecture Notes in Computer Science, páginas 115-126. Springer. (Citado nas páginas 60 e 61.)

Dean, J. e Ghemawat, S. (2004). Mapreduce: Simplified data processing on large clusters. Em Proceedings of the 6th Conference on Symposium on Opearting Systems Design \& Implementation - Volume 6, OSDI'04, páginas 10-10, Berkeley, CA, USA. USENIX Association. (Citado nas páginas 117 e 118.)

Dean, J. e Ghemawat, S. (2008). Mapreduce: Simplified data processing on large clusters. Communications of the ACM, 51(1):107-113. (Citado nas páginas 117 e 118.)

Dempster, A. P., Laird, N. M., e Rubin, D. B. (1977). Maximum likelihood from incomplete data via the em algorithm. Journal of the Royal Statistical Society. Series B (Methodological), 39(1):1-38. (Citado na página 12.)

DeSarbo, W. e Mahajan, V. (1984). Constrained classification: The use of a priori information in cluster analysis. Psychometrika, 49(2):187-215. (Citado na página 59.)

Dima, A. A., Elliott, J. T., Filliben, J. J., Halter, M., Peskin, A., Bernal, J., Kociolek, M., Brady, M. C., Tang, H. C., e Plant, A. L. (2011). Comparison of segmentation algorithms for fluorescence microscopy images of cells. Cytometry Part A, 79A(7):545-559. (Citado na página 65.) 
Eiben, A. E. e Smith, J. E. (2003). Introduction to Evolutionary Computing. Springer. (Citado nas páginas $5,20,31,72$ e 98 .)

Elango, M., Nachiappan, S., e Tiwari, M. K. (2011). Balancing task allocation in multi-robot systems using k-means clustering and auction based mechanisms. Expert Systems with Applications, 38(6):6486-6491. (Citado na página 65.)

Elder, J. F. e Pregibon, D. (1996). A statistical perspective on knowledge discovery in databases. Em Advances in Knowledge Discovery and Data Mining, páginas 83-113. AAAI/MIT Press. (Citado na página 3.)

Everitt, B. S. (2001). Cluster Analysis. Edward Arnold and Halsted Press. (Citado na página 23.)

Falkenauer, E. (1998). Genetic Algorithms and Grouping Problems. John Wiley \& Sons, Inc. (Citado na página 21.)

Fayyad, U. M., Djorgovski, S. G., e Weir, N. (1996a). Automating the analysis and cataloging of sky surveys. Em Advances in Knowledge Discovery and Data Mining, páginas 471-493. AAAI/MIT Press. (Citado na página 1.)

Fayyad, U. M., Piatetsky-Shapiro, G., Smyth, P., e Uthurusamy, R., editores (1996b). Advances in Knowledge Discovery and Data Mining. AAAI/MIT Press. (Citado nas páginas 1 e 2.)

Figueiredo, M. e Jain, A. (2002). Unsupervised learning of finite mixture models. IEEE Transactions on Pattern Analysis and Machine Intelligence, 24(3):381-396. (Citado nas páginas $6,14,36$ e 101.)

Fraley, C. e Raftery, A. E. (1998). How many clusters? which clustering method? answers via model-based cluster analysis. The Computer Journal, 41(8):578-588. (Citado na página 6.)

Fraley, C. e Raftery, A. E. (2002). Model-based clustering, discriminant analysis, and density estimation. Journal of the American Statistical Association, 97:611-631. (Citado na página 6.)

Freitas, A. A. (2002). Data Mining and Knowledge Discovery with Evolutionary Algorithms. Springer-Verlag New York, Inc., Secaucus, NJ, USA. (Citado nas páginas 19, 20 e 21.)

Freund, A., Pelleg, D., e Richter, Y. (2008). Clustering from constraint graphs. Em SIAM International Conference on Data Mining (SDM 2008), páginas 301-312. (Citado na página 60.)

Gama, J. e Gaber, M. (2007). Learning from Data Streams: Processing Techniques in Sensor Networks. New generation computing. Springer. (Citado na página 2.) 
Goldberg, A. B. (2010). New Directions in Semi-supervised Learning. Tese de Doutorado, University of Wisconsin - Madison. (Citado nas páginas 5 e 6.)

Golub, G. H. e Van Loan, C. F. (1996). Matrix computations. Johns Hopkins University Press, Baltimore, MD, USA, 3 edition. (Citado nas páginas 19 e 69.)

Gondek, D. e Hofmann, T. (2004). Non-redundant data clustering. Em IEEE International Conference on Data Mining (ICDM'04), páginas 75-82, Washington, DC, USA. IEEE Computer Society. (Citado na página 60.)

Gordon, A. D. (1973). Classification in the presence of constraints. Biometrics, 29:821-827. (Citado na página 59.)

Graham, R. L., Knuth, D. E., e Patashnik, O. (1994). Concrete Mathematics: A Foundation for Computer Science. Addison-Wesley Longman Publishing Co., Inc., Boston, MA, USA, 2nd edition. (Citado na página 4.)

Greene, D. e Cunningham, P. (2007). Constraint selection by committee: An ensemble approach to identifying informative constraints for semi-supervised clustering. Em European Conference on Machine Learning (ECML 2007), volume 4701 of Lecture Notes in Computer Science, páginas 140-151. Springer. (Citado nas páginas 60 e 61.)

Grira, N., Crucianu, M., e Boujemaa, N. (2006). Fuzzy clustering with pairwise constraints for knowledge-driven image categorisation. IEEE Proceedings - Vision, Image and Signal Processing, 153(3):299 - 304. (Citado na página 62.)

Han, J. e Kamber, M. (2000). Data Mining: Concepts and Techniques. Morgan Kaufmann. (Citado na página 3.)

Hand, D. J., Mannila, H., e Smyth, P. (2001). Principles of Data Mining (Adaptive Computation and Machine Learning). The MIT Press. (Citado nas páginas 2 e 3.)

Hochbaum, D. S. e Shmoys, D. B. (1985). A best possible heuristic for the k-center problem. Mathematics of Operations Research, 10(2):pp. 180-184. (Citado na página 70.)

Hofmann, T. e Buhmann, J. (1998). Competitive learning algorithms for robust vector quantization. Signal Processing, IEEE Transactions on, 46(6):1665 -1675. (Citado na página 77.)

Hong, Y., Kwong, S., Xiong, H., e Ren, Q. (2008). Genetic-guided semi-supervised clustering algorithm with instance-level constraints. Em Conference on Genetic and Evolutionary Computation (GECCO’08), páginas 1381-1388, New York, NY, USA. ACM. (Citado nas páginas $63,66,71$ e 72.$)$ 
Horta, D. e Campello, R. J. G. B. (2009). Fast evolutionary algorithms for relational clustering. Em 9th International Conference on Intelligent Systems Design and Applications (ISDA'09), páginas 1456-1462. IEEE Computer Society. (Citado nas páginas 24, 25, 26 e 35.)

Horta, D., de Andrade, I. C., e Campello, R. J. (2011). Evolutionary fuzzy clustering of relational data. Theoretical Computer Science, In Press, Accepted Manuscript. (Citado na página 26.)

Hruschka, E. R. (2001). Algoritmos Genéticos de Agrupamento para Extração de Regras de Redes Neurais. Tese de Doutorado, Universidade Federal do Rio de Janeiro. (Citado na página 4.)

Hruschka, E. R., Campello, R. J., e de Castro, L. N. (2006). Evolving clusters in geneexpression data. Information Sciences, 176(13):1898 - 1927. (Citado nas páginas 22, 23 e 92.)

Hruschka, E. R., Campello, R. J., Freitas, A., e de Carvalho, A. C. (2009). A survey of evolutionary algorithms for clustering. IEEE Transactions on Systems, Man and Cybernetics, Part C: Applications and Reviews, 39(2):133-155. (Citado nas páginas 5, 15, 20, 21, 22, 23, 27 , 29, 36 e 38.$)$

Hruschka, E. R., de Castro, L. N., e Campello, R. J. G. B. (2004). Evolutionary Algorithms for Clustering Gene-Expression Data. 4th IEEE International Conference on Data Mining (ICDM’04), páginas 403-406. (Citado na página 22.)

Hu, G., Zhou, S., Guan, J., e Hu, X. (2008a). Towards effective document clustering: A constrained k-means based approach. Information Processing and Management, 44:1397-1409. (Citado nas páginas 62, 63 e 65.)

Hu, Y., Wang, J., Yu, N., e Hua, X.-S. (2008b). Maximum margin clustering with pairwise constraints. Em IEEE International Conference on Data Mining (ICDM'08), páginas 253 -262. (Citado na página 62.)

Hubert, L. e Arabie, P. (1985). Comparing partitions. Journal of Classification, 2:193-218. (Citado nas páginas 93 e 109.)

Jain, A. K. (2010). Data clustering: 50 years beyond k-means. Pattern Recognition Letters, 31:651-666. (Citado nas páginas 63, 64 e 65.)

Jain, A. K. e Dubes, R. C. (1988). Algorithms for Clustering Data. Prentice-Hall. (Citado nas páginas 3, 6 e 21.)

Jain, A. K., Murty, M. N., e Flynn, P. J. (1999). Data clustering: a review. ACM Computing Surveys, 31:264-323. (Citado na página 64.) 
Jordan, M. I. e Xu, L. (1995). Convergence results for the em approach to mixtures of experts architectures. Neural Networks, 8(9):1409 - 1431. (Citado na página 6.)

Kantardzic, M. e Zurada, J. (2005). Next Generation of Data-Mining Applications. Wiley-IEEE Press. (Citado na página 1.)

Kaufman, L. e Rousseeuw, P. J. (1990). Finding Groups in Data: An Introduction to Cluster Analysis. John Wiley. (Citado nas páginas 4 e 23.)

Kimbrough, S. O., Koehler, G. J., Lu, M., e Wood, D. H. (2008). On a feasible-infeasible twopopulation (fi-2pop) genetic algorithm for constrained optimization: Distance tracing and no free lunch. European Journal of Operational Research, 190(2):310-327. (Citado na página 98.)

Kimbrough, S. O., Lu, M., Wood, D. H., e Wu, D.-J. (2002). Exploring a two-market genetic algorithm. Em GECCO 2002: Proceedings of the Genetic and Evolutionary Computation Conference, páginas 415-422. Morgan Kaufmann. (Citado na página 98.)

Kimbrough, S. O., Lu, M., Wood, D. H., e Wu, D.-J. (2003). Exploring a two-population genetic algorithm. Em GECCO 2003: Proceedings of the Genetic and Evolutionary Computation Conference, volume 2723 of Lecture Notes in Computer Science, páginas 1148-1159. Springer. (Citado na página 98.)

Kindermann, R., Snell, J., e Society, A. M. (1980). Markov random fields and their applications. Contemporary mathematics. American Mathematical Society. (Citado na página 73.)

Klein, D., Kamvar, S., e Manning, C. (2002). From instance-level constraints to space-level constraints: Making the most of prior knowledge in data clustering. Em International Conference on Machine Learning (ICML'02), páginas 307-314. Morgan Kaufmann. (Citado nas páginas xix, 61, 87 e 88 .)

Kohonen, T. (1990). Improved versions of learning vector quantization. Em IJCNN, páginas 545-550. (Citado nas páginas 77 e 78.)

Koller, D. e Friedman, N. (2009). Probabilistic Graphical Models: Principles and Techniques - Adaptive Computation and Machine Learning. The MIT Press. (Citado na página 73.)

Kononenko, I. (2001). Machine learning for medical diagnosis: history, state of the art and perspective. Artificial Intelligence in Medicine, 23(1):89 - 109. (Citado na página 3.)

Kuhn, H. W. (1955). The Hungarian method for the assignment problem. Naval Research Logistic Quarterly, 2:83-97. (Citado na página 93.) 
Lange, T., Law, M., Jain, A., e Buhmann, J. (2005). Learning with constrained and unlabelled data. Em IEEE Conference on Computer Vision and Pattern Recognition (CVPR'05), volume 1, páginas 731-738. (Citado nas páginas 61, 63 e 73.)

Law, M. H. C., Topchy, A. P., e Jain, A. K. (2005). Model-based clustering with probabilistic constraints. Em SIAM International Conference on Data Mining (SDM'05), páginas 641645. SIAM. (Citado nas páginas 62, 63 e 73.)

LeCun, Y., Bottou, L., Bengio, Y., e Haffner, P. (1998). Gradient-based learning applied to document recognition. Proceedings of the IEEE, 86(11):2278-2324. (Citado na página 118.)

Letrémy, P., Cottrell, M., Esposito, E., Laffite, V., e Showk, S. (2007). Consumer Profile Identification and Allocation. Em Francisco Sandoval, Alberto Prieto, Joan Cabestany, e Manuel Grana, editores, Computational and Ambient Intelligence - 9th International WorkConference on Artificial Neural Networks, (IWANN'07), Lecture Notes in Computer Science - 4507, páginas 530-538. Springer. (Citado na página 5.)

Li, L. e Ma, J. (2008). A BYY Split-and-Merge EM Algorithm for Gaussian Mixture Learning. Em 5th international Symposium on Neural Networks: Advances in Neural Networks (ISNN’08), páginas 600-609. Springer-Verlag. (Citado nas páginas 16 e 40.)

Li, Y. e Li, L. (2009). A novel split and merge em algorithm for gaussian mixture model. Em International Conference on Natural Computation (ICNC'09), páginas 479-483, Washington, DC, USA. IEEE Computer Society. (Citado nas páginas 17, 18 e 19.)

Li, Y. e Zemel, R. (2014). High order regularization for semi-supervised learning of structured output problems. Em International Conference on Machine Learning (ICML). (Citado na página 6.)

Li, Z., Liu, J., e Tang, X. (2008). Pairwise constraint propagation by semidefinite programming for semi-supervised classification. Em International Conference on Machine learning (ICML’08), páginas 576-583, New York, NY, USA. ACM. (Citado na página 61.)

Li, Z., Liu, J., e Tang, X. (2009). Constrained clustering via spectral regularization. Em IEEE Conference on Computer Vision and Pattern Recognition (CVPR'09), páginas 421-428. (Citado na página 62.)

Lin, L. e Wang, S. (2005). Genetic algorithms and fuzzy approach to gaussian mixture model for speaker recognition. Em IEEE International Conference on Natural Language Processing and Knowledge Engineering (IEEE NLP-KE'05), páginas 142 - 146. (Citado na página 26.)

Liu, C. L. (1968). Introduction to Combinatorial Mathematics (Computer Science Series). Mcgraw-Hill College. (Citado na página 4.) 
Liu, S.-T. e Tsai, W.-S. (1989). Moment-preserving clustering. Pattern Recognition, 22:433447. (Citado na página 15.)

Lu, Z. e Leen, T. K. (2007a). Penalized probabilistic clustering. Neural Computation, 19:15281567. (Citado nas páginas 62, 63 e 73.)

Lu, Z. e Leen, T. K. (2007b). Semi-supervised clustering with pairwise constraints: A discriminative approach. Em International Conference on Artificial Intelligence and Statistics (AISTATS'07), páginas 1-8. (Citado na página 62.)

Lu, Z. e Peng, Y. (2008). A semi-supervised learning algorithm on gaussian mixture with automatic model selection. Neural Processing Letters, 27:57-66. (Citado na página 61.)

Ma, J. e He, Q.-C. (2005). A dynamic merge-or-split learning algorithm on gaussian mixture for automated model selection. Em Intelligent Data Engineering and Automated Learning (IDEAL'05), Lecture Notes in Computer Science, páginas 203-210. Springer. (Citado nas páginas $15,17,18$ e 19.)

Mahajan, V. e Jain, A. K. (1978). An approach to normative segmentation. Journal of Marketing Research, 15(3):pp. 338-345. (Citado na página 59.)

Maitra, R. (2009). Initializing partition-optimization algorithms. IEEE/ACM Trans. Comput. Biol. Bioinformatics, 6(1):144-157. (Citado na página 49.)

Maitra, R. e Melnykov, V. (2010). Simulating data to study performance of finite mixture modeling and clustering algorithms. Journal of Computational and Graphical Statistics, 19(2):354-376. (Citado nas páginas 48 e 49.)

Martínez, A. M. e Virtriá, J. (2000). Learning mixture models using a genetic version of the EM algorithm. Pattern Recognition Letters, 21:759-769. (Citado na página 26.)

McLachlan, G. e Peel, D. (2000). Finite Mixture Models. Wiley Series in Probability and Statistics. Wiley-Interscience, 1 edition. (Citado nas páginas 6, 11, 12, 13, 14, 36 e 63.)

McLachlan, G. J. e Krishnan, T. (1997). The EM algorithm and extensions. Wiley, New York. (Citado nas páginas 11, 12, 13, 14 e 28.)

Medeiros, D. M. R. (2010). Análise de dados por meio de agrupamento fuzzy semisupervisionado e mineração de textos. Tese de Doutorado, Universidade de São Paulo. (Citado na página 62.)

Messina, A. e Langer, H. (2011). Pattern recognition of volcanic tremor data on mt. etna (italy) with kkanalysis-a software program for unsupervised classification. Computers \& Geosciences, 37(7):953-961. (Citado na página 65.) 
Michalewicz, Z. (1995). A survey of constraint handling techniques in evolutionary computation methods. Em Proceedings of the 4th Annual Conference on Evolutionary Programming, páginas 135-155. MIT Press. (Citado na página 98.)

Michalewicz, Z. e Nazhiyath, G. (1995). Genocop iii: a co-evolutionary algorithm for numerical optimization problems with nonlinear constraints. Em Evolutionary Computation, 1995., IEEE International Conference on, volume 2, páginas 647-651 vol.2. (Citado na página 98.)

Milligan, G. (1996). Clustering validation: Results and implications for applied analyses. Em Clustering and Classification, páginas 341-373. World Scientific Publishing Company. (Citado na página 4.)

Naldi, M. C. (2011). Técnicas de combinação para agrupamento centralizado e distribuído de dados. Tese de Doutorado, Universidade de São Paulo. (Citado nas páginas 21 e 25.)

Naldi, M. C., Campello, R. J., Hruschka, E. R., e Carvalho, A. C. (2011). Efficiency issues of evolutionary k-means. Applied Soft Computing, 11(2):1938 - 1952. (Citado nas páginas 14, $22,24,25,26,35,36$ e 54 .)

Nguyen, C. D. e Cios, K. J. (2008). GAKREM: A novel hybrid clustering algorithm. Information Sciences, 178(22):4205 - 4227. (Citado nas páginas 27, 29, 31, 32, 36 e 47.)

Nigam, K., McCallum, A., Thrun, S., e Mitchell, T. M. (2000). Text classification from labeled and unlabeled documents using em. Machine Learning, 39(2/3):103-134. (Citado na página 6.)

Niknam, T. e Amiri, B. (2010). An efficient hybrid approach based on pso, aco and k-means for cluster analysis. Applied Soft Computing, 10(1):183-197. (Citado na página 22.)

Nisbet, R., Elder, J., e Miner, G. (2009). Handbook of Statistical Analysis and Data Mining Applications. Academic Press. (Citado na página 1.)

Opper, M. e Saad, D. (2001). Advanced mean field methods: theory and practice. Neural information processing series. MIT Press. (Citado na página 73.)

Oyama, S. e Tanaka, K. (2006). Learning a distance metric for object identification without human supervision. Em Knowledge Discovery in Databases: 10th European Conference on Principles and Practice of Knowledge Discovery in Databases (PKDD’06), páginas 609616. Springer. (Citado na página 6.)

Patra, B. (2011). Convergence of distributed asynchronous learning vector quantization algorithms. JMLR, 12:3431-3466. (Citado nas páginas 77 e 78 .) 
Pelleg, D. e Baras, D. (2007). $K$-means with large and noisy constraint sets. Em European Conference on Machine Learning (ECML'07), páginas 674-682. (Citado nas páginas 62, 63, $66,67,68,83$ e 86.$)$

Pernkopf, F. e Bouchaffra, D. (2005). Genetic-based EM algorithm for learning Gaussian mixture models. IEEE Transactions on Pattern Analysis and Machine Intelligence, 27:13441348. (Citado nas páginas 14, 27, 29, 30, 36, 37, 38, 42, 47, 48, 49, 54, 58 e 116.)

Petricoin III, E. F., Ardekani, A. M., Hitt, B. A., Levine, P. J., Fusaro, V. A., Steinberg, S. M., Mills, G. B., Simone, C., Fishman, D. A., Kohn, E. C., e Liotta, L. A. (2002). Use of proteomic patterns in serum to identify ovarian cancer. The Lancet, 359(9306):572 - 577. (Citado na página 92.)

Phillips, H. L., Walker, P. B., Kennedy, C. H., Carmichael, O., e Davidson, I. N. (2013). Guided learning algorithms: An application of constrained spectral partitioning to functional magnetic resonance imaging (fmri). Em Schmorrow, D. e Fidopiastis, C., editores, Foundations of Augmented Cognition, volume 8027 of Lecture Notes in Computer Science, páginas 709-716. Springer Berlin Heidelberg. (Citado na página 60.)

Piatetsky-Shapiro, G. e Frawley, W. J., editores (1991). Knowledge Discovery in Databases. AAAI/MIT Press. (Citado na página 1.)

Provost, F. e Fawcett, T. (2013). Data Science for Business: What You Need to Know About Data Mining and Data-analytic Thinking. O’Reilly Media, Inc., 1st edition. (Citado na página 1.)

Quinlan, J. R. (1993). C4.5: programs for machine learning. Morgan Kaufmann Publishers Inc., San Francisco, CA, USA. (Citado na página 3.)

Raghuram, J., Miller, D., e Kesidis, G. (2014). Instance-level constraint-based semisupervised learning with imposed space-partitioning. Neural Networks and Learning Systems, IEEE Transactions on, 25(8):1520-1537. (Citado nas páginas 7, 9, 62, 64, 73, 74, 75, 97, 108, 109, 113 e 117.)

Rasmussen, C. E. (2000). The infinite gaussian mixture model. Em In Advances in Neural Information Processing Systems 12, páginas 554-560. MIT Press. (Citado na página 11.)

Redner, R. A. e Walker, H. F. (1984). Mixture densities, maximum likelihood and the em algorithm. SIAM Review, 26(2):195-239. (Citado na página 38.)

Reynolds, D. e Rose, R. (1995). Robust text-independent speaker identification using gaussian mixture speaker models. IEEE Transactions on Speech and Audio Processing, 3(1):72 -83. (Citado na página 12.) 
Rissanen, J. (1989). Stochastic Complexity in Statistical Inquiry Theory. World Scientific Publishing Co., Inc. (Citado nas páginas 27 e 36.)

Rokach, L. e Maimon, O. (2008). Data Mining with Decision Trees: Theroy and Applications. World Scientific Publishing Co., Inc., River Edge, NJ, USA. (Citado na página 1.)

Rothaus, K. e Jiang, X. (2008). Constrained clustering by a novel graph-based distance transformation. Em 19th International Conference on Pattern Recognition (ICPR'08), páginas 1-4. (Citado nas páginas 61, 63 e 65.)

Schwarz, G. (1978). Estimating the dimension of a model. Annals of Statistics, 6:461-464. (Citado na página 73.)

Sestaro, D., Covoes, T., e Hruschka, E. (2012). A semi-supervised approach to estimate the number of clusters per class. Em Neural Networks (SBRN), 2012 Brazilian Symposium on, páginas 73-78. (Citado nas páginas 8, 9, 88 e 95.)

Sevon, P., Toivonen, H., e Onkamo, P. (2005). Gene mapping by pattern discovery. Em Data Mining in Bioinformatics, páginas 105-126. Springer. (Citado na página 1.)

Shalev-Shwartz, S. (2012). Online learning and online convex optimization. Foundations and Trends in Machine Learning, 4(2):107-194. (Citado na página 2.)

Shental, N., Bar-Hillel, A., Hertz, T., e Weinshall, D. (2003). Computing gaussian mixture models with em using equivalence constraints. Em Advances in Neural Information Processing Systems 16 (NIPS’03), páginas 465-472. MIT Press. (Citado nas páginas 61, 63, 73 e 97.)

Silva, J. A., Faria, E. R., Barros, R. C., Hruschka, E. R., Carvalho, A. C. P. L. F. d., e Gama, J. a. (2013). Data stream clustering: A survey. ACM Comput. Surv., 46(1):13:1-13:31. (Citado na página 2.)

Silva, J. d. A. e Hruschka, E. R. (2009). EACImpute: An evolutionary algorithm for clusteringbased imputation. Em 9th International Conference on Intelligent Systems Design and Applications (ISDA’09), páginas 1400-1406. IEEE Computer Society. (Citado na página 26.)

Silva, J. d. A. S. e Hruschka, E. R. (2013). An experimental study on the use of nearest neighbor-based imputation algorithms for classification tasks. Data \& Knowledge Engineering, 84(0):47 - 58. (Citado na página 26.)

Silva, T. C. e Liang, Z. (2012). Stochastic competitive learning in complex networks. IEEE Trans. Neural Netw. Learning Syst., páginas 385-398. (Citado na página 77.)

Steinbach, M., Karypis, G., e Kumar, V. (2000). A comparison of document clustering techniques. Em KDD Workshop on Text Mining. (Citado na página 39.) 
Steinley, D. (2006). K-means clustering: a half-century synthesis. British Journal of Mathematical and Statistical Psychology, 59:1-34. (Citado nas páginas 63, 64 e 65.)

Strehl, A. e Ghosh, J. (2003). Cluster ensembles - a knowledge reuse framework for combining multiple partitions. JMLR, 3:583-617. (Citado na página 83.)

Tan, P.-N., Steinbach, M., e Kumar, V. (2005). Introduction to Data Mining. Addison-Wesley. (Citado na página 39.)

Tawfick, M. M., Abbas, H. M., e Shahein, H. I. (2008). An integer-coded evolutionary approach for mixture maximum likelihood clustering. Pattern Recognition Letters, 29:515-524. (Citado na página 27.)

Thang, N. D., Lihui, C., e Keong, C. C. (2009). An outlier-aware data clustering algorithm in mixture models. Em International Conference on Information, Communications and Signal Processing (ICICS'09), páginas 1 -5. (Citado na página 27.)

Theodoridis, S. e Koutroumbas, K. (2006). Pattern Recognition. Academic Press. (Citado nas páginas 2 e 4.)

Tohka, J., Krestyannikov, E., Dinov, I., Graham, A., Shattuck, D., Ruotsalainen, U., e Toga, A. (2007). Genetic algorithms for finite mixture model based voxel classification in neuroimaging. IEEE Transactions on Medical Imaging, 26(5):696 -711. (Citado nas páginas 12, 27 e 118.)

Ueda, N. e Nakano, R. (1998). Deterministic annealing EM algorithm. Neural Networks, 11(2):271 - 282. (Citado nas páginas 14, 48 e 49.)

Ueda, N., Nakano, R., Ghahramani, Z., e Hinton, G. E. (2000). SMEM algorithm for mixture models. Neural Comput., 12:2109-2128. (Citado nas páginas 14, 15, 16, 17, 18 e 40.)

Črepinšek, M., Liu, S.-H., e Mernik, M. (2013). Exploration and exploitation in evolutionary algorithms: A survey. ACM Computing Surveys, 45(3):35:1-35:33. (Citado na página 38.)

Verbeek, J. J., Vlassis, N. A., e Kröse, B. J. A. (2003). Efficient greedy learning of gaussian mixture models. Neural Computation, 15(2):469-485. (Citado na página 14.)

Vinh, N. X., Epps, J., e Bailey, J. (2010). Information theoretic measures for clusterings comparison: Variants, properties, normalization and correction for chance. Journal of Machine Learning Research, 11:2837-2854. (Citado nas páginas 93 e 109.)

Vu, V.-V., Labroche, N., e Bouchon-Meunier, B. (2010). Boosting clustering by active constraint selection. Em European Conference on Artificial Intelligence (ECAI 2010), Frontiers in Artificial Intelligence and Applications, páginas 297-302. IOS Press. (Citado nas páginas 60 e 61.) 
Wagstaff, K. e Cardie, C. (2000). Clustering with instance-level constraints. Em International Conference on Machine Learning (ICML'00), páginas 1103-1110, San Francisco, CA, USA. Morgan Kaufmann Publishers Inc. (Citado nas páginas 59 e 61.)

Wagstaff, K., Cardie, C., Rogers, S., e Schroedl, S. (2001). Constrained k-means clustering with background knowledge. Em International Conference on Machine Learning (ICML 2001), páginas 577-584. Morgan Kaufmann. (Citado nas páginas 6, 59, 60, 61, 62, 63, 65, 66, 89 e 92.)

Wang, H. X., Luo, B., Zhang, Q. B., e Wei, S. (2004). Estimation for the number of components in a mixture model using stepwise split-and-merge em algorithm. Pattern Recognition Letters, 25:1799-1809. (Citado nas páginas 16, 17, 18 e 40.)

Wang, J., Jebara, T., e Chang, S.-F. (2013). Semi-supervised learning using greedy max-cut. Journal of Machine Learning Research, 14(1):771-800. (Citado na página 6.)

Wang, X., Qian, B., e Davidson, I. (2014). On constrained spectral clustering and its applications. Data Mining and Knowledge Discovery, 28(1):1-30. (Citado na página 62.)

Witten, I. H. e Frank, E. (2005). Data Mining: Practical Machine Learning Tools and Techniques. Morgan Kaufmann Series in Data Management Systems. Morgan Kaufmann. (Citado na página 3.)

Wu, X., Kumar, V., Ross, Ghosh, J., Yang, Q., Motoda, H., McLachlan, G., Ng, A., Liu, B., Yu, P., Zhou, Z.-H., Steinbach, M., Hand, D., e Steinberg, D. (2008). Top 10 algorithms in data mining. Knowledge and Information Systems, 14(1):1-37. (Citado na página 64.)

Xing, E. P., Ng, A. Y., Jordan, M. I., e Russell, S. (2002). Distance metric learning, with application to clustering with side-information. Em Advances in Neural Information Processing Systems 15 (NIPS'02), páginas 505-512. MIT Press. (Citado nas páginas 62, 69 e 70.)

Xu, L., Krzyzak, A., e Oja, E. (1993). Rival penalized competitive learning for clustering analysis, RBF net, and curve detection. IEEE Transactions on Neural Networks, 4(4):636 -649. (Citado nas páginas 78, 79 e 81.)

Xu, R. e Wunsch, D. (2009). Clustering. Wiley-IEEE Press. (Citado nas páginas 3, 21, 63 e 65.)

Yeung, K., Medvedovic, M., e Bumgarner, R. (2003). Clustering gene-expression data with repeated measurements. Genome Biology, 4(5):R34. (Citado na página 5.)

Zeng, E., Yang, C., Li, T., e Narasimhan, G. (2007). On the effectiveness of constraints sets in clustering genes. Em IEEE International Conference on Bioinformatics and Bioengineering, páginas 79-86. (Citado na página 60.) 
Zhang, B., Zhang, C., e Yi, X. (2004a). Competitive EM algorithm for finite mixture models. Pattern Recognition, 37(1):131-144. (Citado nas páginas 17, 18 e 40.)

Zhang, L., Zhu, J., e Yao, T. (2004b). An evaluation of statistical spam filtering techniques. ACM Transactions on Asian Language Information Processing (TALIP), 3(4):243-269. (Citado na página 3.)

Zhang, S. e Wong, H.-S. (2009). Active constrained clustering with multiple cluster representatives. Em IEEE International Conference on Systems, Man and Cybernetics (SMC'09), páginas 2689 -2694. (Citado nas páginas 62, 63 e 65.)

Zhang, Z., Chen, C., Sun, J., e Chan, K. L. (2003). EM algorithms for Gaussian mixtures with split-and-merge operation. Pattern Recognition, 36(9):1973 - 1983. (Citado nas páginas 12, $14,15,17,18,19,38,40,42,45,50,116$ e 118.$)$

Zhao, Q. e Miller, D. J. (2005). Mixture modeling with pairwise, instance-level class constraints. Neural Computation, 17:2482-2507. (Citado nas páginas 7, 62, 63, 73, 75, 97 e 108.) 